\title{
Macrocycloadditions leading to conformationally restricted small molecules **
}

\author{
Ryan E. Looper, Daniela Pizzirani and Stuart L. Schreiber* \\ Contribution from the Program in Chemical Biology, Broad Institute of Harvard and MIT, and the \\ Howard Hughes Medical Institute, Department of Chemistry and Chemical Biology, Harvard \\ University, Cambridge, Massachusetts 02138
}

\section{Supporting Information}

\section{Contents:}

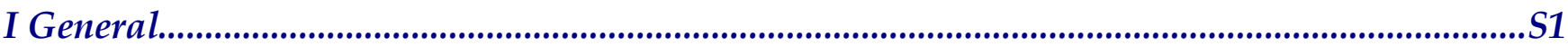

II General procedure A for the preparation of the ketoimides.................................................. 2

III General procedure B for the diazotization of the ketoimides................................................S2

IV General procedure $C$ for the $\mathrm{Rh}(\mathrm{II})$ catalyzed cycloaddition-cycloreversion sequence............S3

$V$ General procedure $D$ for the deprotection and coupling of the allyl ester................................S7

VI General procedure $E$ for the $\mathrm{Cu}(\mathrm{I})$ catalyzed macrocycloaddition reaction............................S12

VII General procedure $F$ for the acyclic cycloaddition reactions..............................................S18

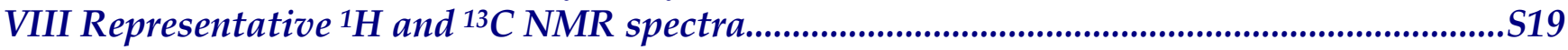

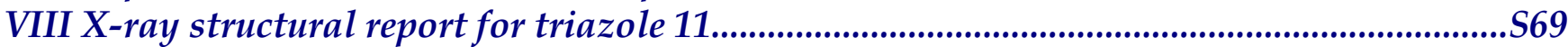

\section{General}

Dry solvents were dispensed from a solvent purification system that passes solvents through packed columns (THF and $\mathrm{CH}_{2} \mathrm{Cl}_{2}$ : dry neutral alumina; toluene: dry neutral alumina and Q5 reactant; DMF: activated molecular sieves). Unless otherwise stated, all reagents were obtained from commercial sources and used without further purification. ${ }^{1} \mathrm{H}$ NMR spectra were recorded on Varian Mercury $400(400 \mathrm{MHz})$, Bruker Avance DMX500 (500MHz), or Varian Unity/Inova $600(600 \mathrm{MHz})$ spectrometers. ${ }^{1} \mathrm{H}$ data are reported as follows: chemical shift in parts per million relative to $\mathrm{CHCl}_{3}(7.26 \mathrm{ppm})$ multiplicity ( $\mathrm{s}$, singlet; $\mathrm{d}$, doublet; $\mathrm{t}$, triplet; q, quartet; $\mathrm{m}$, multiplet; br, broadened and exch, exchangeable), coupling constant $(\mathrm{Hz})$, and integration. ${ }^{13} \mathrm{C}$ magnetic resonance spectra were recorded on Varian Mercury $400(100 \mathrm{MHz})$ or Varian Unity/Inova $500(125 \mathrm{MHz})$ spectrometers. ${ }^{13} \mathrm{C}$ chemical shifts are reported in parts per million relative to solvent. All ${ }^{13} \mathrm{C}$ spectra were determined with broadband decoupling. All reactions were monitored by thin layer chromatography using glass plates precoated with Merck silica gel 60 F254. Flash chromatography was performed either on EM Science silica gel 60 (230-400 mesh) or using a CombiFlash Companion system (Teledyne ISCO, Inc.) with pre-packed FLASH silica gel columns (Biotage, Inc.). 
II General procedure A for the preparation of the ketoimides:

(S) and (R)-allyl 3-oxo-3-(2-oxo-5-((triisopropylsilyloxy)methyl)pyrrolidin-1-yl)propanoate (2a). To a solution of the pyrrolidinone (S)-1 $(4.18 \mathrm{~g}, 15.4 \mathrm{mmol})$ in $\mathrm{CH}_{2} \mathrm{Cl}_{2}$ $(40 \mathrm{~mL})$ was added $\mathrm{Et}_{3} \mathrm{~N}(8.2 \mathrm{~mL}, 58.5 \mathrm{mmol}, 3.8 \mathrm{eq})$ and the mixture cooled to $0{ }^{\circ} \mathrm{C}$. TMSCl $(6.84 \mathrm{~mL}, 53.9 \mathrm{mmol}, 3.5 \mathrm{eq})$ was added dropwise and the mixture stirred for 1h. A solution of Meldrum's acid (2.44 g, 16.9

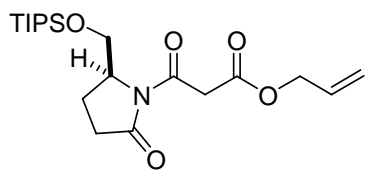
$\mathrm{mmol}, 1.1 \mathrm{eq}$ ) was then added and the mixture warmed to $\mathrm{rt}$ and stirred for $1.5 \mathrm{~h}$. The mixture was diluted with EtOAc $(100 \mathrm{~mL})$, washed with $10 \% \mathrm{HCl}(100 \mathrm{~mL})$ and dried $\left(\mathrm{Na}_{2} \mathrm{SO}_{4}\right)$. This solution was passed through a short silica plug and concentrated. The residue was taken up in $\mathrm{CH}_{2} \mathrm{Cl}_{2}(40 \mathrm{~mL})$ and cooled to $0{ }^{\circ} \mathrm{C}$. Allyl alcohol $(2.06 \mathrm{~mL}, 30.8 \mathrm{mmol}, 2 \mathrm{eq})$ was then added along with DMAP $(188 \mathrm{mg}, 0.1 \mathrm{eq})$. DIC $(2.34 \mathrm{~mL}, 1.1 \mathrm{eq})$ was then added and the mixture stirred for $12 \mathrm{~h}$. The mixture was again concentrated, diluted with 6:1 Hexanes : EtOAC (to remove $N, N^{\prime}$-diisopropylurea) and passed through another short silica plug, re-concentrated and purified on silica eluting with 6:1 Hexanes : EtOAc to give the allyl ester as a clear oil $(4.83 \mathrm{~g}, 79 \%)$. (S) $-2 a[\alpha]{ }^{25} \mathrm{D}=-81.6\left(\mathrm{c}=0.51, \mathrm{CH}_{2} \mathrm{Cl}_{2}\right) ;(R)-2 a[\alpha]{ }^{25} \mathrm{D}=+73.8\left(\mathrm{c}=0.53, \mathrm{CH}_{2} \mathrm{Cl}_{2}\right)$. ${ }^{1} \mathrm{H}$ NMR ( $\left.\mathrm{CDCl}_{3}, \mathbf{4 0 0 M H z}\right): \delta 5.89$ (dddd, $\left.J=5.2,10,11,16 \mathrm{~Hz}, 1 \mathrm{H}\right) ; 5.25$ (ddd, $J=1.6,1.6,16$ $\mathrm{Hz}, 1 \mathrm{H}), 5.23(\mathrm{ddd}, J=2.8,2.8,10 \mathrm{~Hz}, 1 \mathrm{H}) ; 4.62(\mathrm{br} \mathrm{d}, J=5.6 \mathrm{~Hz}, 1 \mathrm{H}) ; 4.48-4.45(\mathrm{~m}, 1 \mathrm{H}), 4.08$ $(1 / 2 \mathrm{ABq}, J=16 \mathrm{~Hz}, 1 \mathrm{H}) ; 4.04(\mathrm{dd}, J=3.6,10 \mathrm{~Hz}, 1 \mathrm{H}) ; 3.78(1 / 2 \mathrm{ABq}, J=16 \mathrm{~Hz}, 1 \mathrm{H}), 3.78$ (buried dd, $J=2,10 \mathrm{~Hz}, 1 \mathrm{H}), 2.85$ (ddd, $J=10,10,18 \mathrm{~Hz}, 1 \mathrm{H}) ; 2.44(\mathrm{ddd}, J=2.8,6,18 \mathrm{~Hz}, 1 \mathrm{H})$; 2.20 -2.09 (m, 2H), 1.08-1.00 (m, 21H). ${ }^{13} \mathrm{C}$ NMR (CDCl $\left.3,100 \mathrm{MHz}\right): \delta 176.9,167.1,166.5,131.9$, 118.7, 66.1, 64.2, 58.4, 44.5, 32.9, 21.3, 18.1, 12.1. IR (Dep $\mathbf{C D C l}_{3}$ ): 2944, 2866 (both m), 1747, 1695 (both s), 1365, 1335, 1199 (all m). HRMS (ESI+): Calc. for $\mathrm{C}_{20} \mathrm{H}_{36} \mathrm{NO}_{5} \mathrm{Si}(\mathrm{m} / \mathrm{z}$ ) 398.2363; found $(m / z) 398.2370$.

(S) -prop-2-ynyl-3-oxo-3-(2-oxo-5-((triisopropylsilyloxy)methyl) pyrrolidin-1-yl)propanoate (2b). Prepared according to General procedure A with the pyrrolidinone $(1.00 \mathrm{~g}, 3.69 \mathrm{mmol})$ and propargyl alcohol, yielded the propargyl alcohol as a colorless oil $(1.06 \mathrm{~g}, 73 \%)$. (S)-2b $[\alpha]^{25} \mathrm{D}=+32.6\left(\mathrm{c}=0.34, \mathrm{CH}_{2} \mathrm{Cl}_{2}\right) .{ }^{1} \mathrm{H}$ NMR $\left(\mathrm{CDCl}_{3}, 400 \mathrm{MHz}\right): \delta 4.74(\mathrm{dd}, J=2,16 \mathrm{~Hz}, 1 \mathrm{H}) ; 4.69(\mathrm{dd}, J=2,16$

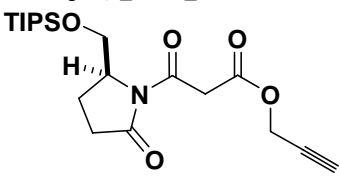
$\mathrm{Hz}, 1 \mathrm{H}) ; 4.49-4.45(\mathrm{~m}, 1 \mathrm{H}) ; 4.08(1 / 2 \mathrm{ABq}, J=16.8 \mathrm{~Hz}, 1 \mathrm{H}) ; 4.04(\mathrm{dd}, J=3.6,10.8 \mathrm{~Hz}, 1 \mathrm{H}) ; 3.81$ $(1 / 2 \mathrm{ABq}, J=16.8 \mathrm{~Hz}, 1 \mathrm{H}) ; 3.78(\mathrm{dd}, J=2,10.8 \mathrm{~Hz}, 1 \mathrm{H}) ; 2.85(\mathrm{ddd}, J=10,10,18 \mathrm{~Hz}, 1 \mathrm{H}) ; 2.49-$ 2.41 (buried $\mathrm{m}, 1 \mathrm{H}) ; 2.47$ (app t, $J=2 \mathrm{~Hz}, 1 \mathrm{H}) ; 2.22-2.09$ (m, 2H); 1.10-1.00 (m, 21H). ${ }^{13} \mathrm{C}$ NMR $\left(\mathrm{CDCl}_{3}, 100 \mathrm{MHz}\right): \delta 176.9,166.6,166.1,77.5,75.4,64.1,58.4,52.9,44.3,32.8,21.3,18.1 .12 .0$. IR (Dep CDCl C: $_{3}$ 2943, 2866, 1747, 1695 (all s). HRMS (ESI+): Calc. for $\mathrm{C}_{20} \mathrm{H}_{34} \mathrm{NO}_{5} \mathrm{Si}(\mathrm{m} / \mathrm{z}$ ) 396.2206; found $(\mathrm{m} / \mathrm{z}) 396.2205$.

III General procedure B for the diazotization of the ketoimides

(S)- and (R)- Allyl diazoimide (3a). To a solution of the ester (S)-2a (3.54 $\mathrm{g}, 8.90 \mathrm{mmol})$ in $\mathrm{MeCN}(20 \mathrm{~mL})$ was added $\mathrm{MsN}_{3}(829 \mu \mathrm{L}, 9.79 \mathrm{mmol}$, $1.1 \mathrm{eq})$ and $\mathrm{Et}_{3} \mathrm{~N}$ (1.50 mL, $10.7 \mathrm{mmol}, 1.2 \mathrm{eq}$.). The mixture was stirred overnight and concentrated. The residue was purified on silica gel

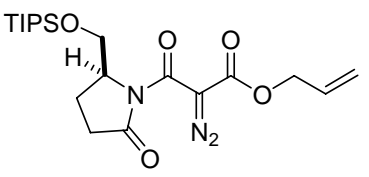
eluting with 5-7\% Acetone/Hexanes to give the diazoimide as a light yellow oil (3.62g, 96\%). $(S)-3 a[\alpha]^{25} \mathrm{D}=-113.2\left(\mathrm{c}=0.50, \mathrm{CH}_{2} \mathrm{Cl}_{2}\right) ;(\boldsymbol{R})-3 a[\alpha]^{25} \mathrm{D}=+96.6\left(\mathrm{c}=0.50, \mathrm{CH}_{2} \mathrm{Cl}_{2}\right) .{ }^{1} \mathbf{H} \mathbf{N M R}$ 
$\left(\mathrm{CDCl}_{3}, 400 \mathrm{MHz}\right): \delta 5.92$ (dddd, $\left.J=5.6,10,11,18 \mathrm{~Hz}, 1 \mathrm{H}\right) ; 5.35(\mathrm{ddd}, J=2,2,18 \mathrm{~Hz}, 1 \mathrm{H}), 5.25$ $(\mathrm{ddd}, J=2,2,10 \mathrm{~Hz}, 1 \mathrm{H}) ; 4.70(\mathrm{ddd}, J=2,2,5.6 \mathrm{~Hz}, 1 \mathrm{H}) ; 4.48-4.45(\mathrm{~m}, 1 \mathrm{H}), 4.06(\mathrm{dd}, J=4,10$ $\mathrm{Hz}, 1 \mathrm{H}) ; 3.78(\mathrm{dd}, J=2.8,10 \mathrm{~Hz}, 1 \mathrm{H}), 2.72(\mathrm{ddd}, J=9,9,18 \mathrm{~Hz}, 1 \mathrm{H}) ; 2.46(\mathrm{ddd}, J=4.4,9,18 \mathrm{~Hz}$, 1H); $2.21-2.08(\mathrm{~m}, 2 \mathrm{H}), 1.08-1.00(\mathrm{~m}, 21 \mathrm{H}) .{ }^{13} \mathrm{C}$ NMR ( $\left.\mathrm{CDCl}_{3}, 100 \mathrm{MHz}\right): \delta 175.2,161.0,160.9$,

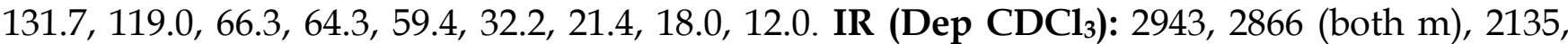
1739, 1322 (all s). HRMS (ESI+): Calc. for $\mathrm{C}_{20} \mathrm{H}_{34} \mathrm{~N}_{3} \mathrm{O}_{5} \mathrm{Si}(\mathrm{m} / \mathrm{z})$ 424.2267; found $(\mathrm{m} / \mathrm{z})$ 422.2265.

Propargyl diazoimide (3b). Prepared according to general procedure B with the ester $(S)-2 b(271 \mathrm{mg}, 0.69 \mathrm{mmol})$ and purified on silica eluting with $7 \%$ Acetone/Hexanes to give the diazoimide $3 \mathbf{b}$ as a light yellow oil (271 mg, 93\%). (S)-3b $[\alpha]{ }^{25} \mathrm{D}=-133.4\left(\mathrm{c}=0.61, \mathrm{CH}_{2} \mathrm{Cl}_{2}\right) .{ }^{1} \mathbf{H}$

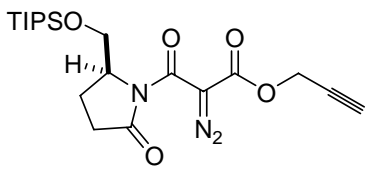
NMR (CDCl $3,400 \mathrm{MHz}): \delta 4.78(\mathrm{t}, J=2 \mathrm{~Hz}, 2 \mathrm{H}), 4.42(\mathrm{dddd}, J=3,4,9,9 \mathrm{~Hz}, 1 \mathrm{H}), 4.08(\mathrm{dd}, J=4$, $10 \mathrm{~Hz}, 1 \mathrm{H}), 3.81(\mathrm{dd}, J=3,10 \mathrm{~Hz}, 1 \mathrm{H}), 2.73(\mathrm{ddd}, J=9,9,18 \mathrm{~Hz}, 1 \mathrm{H}), 2.48(\mathrm{ddd}, J=4,12,18 \mathrm{~Hz}$, $1 \mathrm{H}), 2.49(\mathrm{t}, J=2 \mathrm{~Hz}, 1 \mathrm{H}), 2.24-2.08(\mathrm{~m}, 2 \mathrm{H}), 1.05(\mathrm{~m}, 21 \mathrm{H}) .{ }^{13} \mathrm{C}$ NMR $\left(\mathrm{CDCl}_{3}, 100 \mathrm{MHz}\right)$ : $\delta$ 175.3, 160.6, 125.1, 77.2, 64.3, 59.5, 53.1, 32.3, 21.4, 18.0, 12.0. IR (Dep CDCl CD $_{2}$ 2944, 2866, 2138, 1739, 1652, 1323 (all s). HRMS (ESI+): Calc. for $\mathrm{C}_{20} \mathrm{H}_{32} \mathrm{~N}_{3} \mathrm{O}_{5} \mathrm{Si}(\mathrm{m} / \mathrm{z})$ 422.2111; found $(\mathrm{m} / \mathrm{z})$ 422.2117.

IV General procedure $C$ for the $\mathrm{Rh}(\mathrm{II})$ catalyzed cycloaddition-cycloreversion sequence.

\section{3,4-dimethyl 2-prop-2-ynyl 5-((S)-3-(3-((S)-1-azido-3-phenylpropan-2-yl)ureido)-4-} (triisopropylsilyloxy)butyl)furan-2,3,4-tricarboxylate (4a).

To a solution of the diazoimide (S)-3b $(186 \mathrm{mg}, 0.44 \mathrm{mmol})$ in $\mathrm{PhH} \quad(5 \mathrm{~mL})$ under $\mathrm{N}_{2}$ was added dimethyl acetylenedicarboxylate $\left(57 \mu \mathrm{L}, 0.46 \mathrm{mmol}, 1.05\right.$ eq.) and $\mathrm{Rh}_{2} \mathrm{Oct}_{4}$

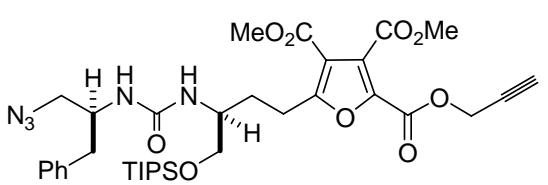
(7 mg, $8.8 \mu \mathrm{mol}[1-10 \mathrm{~mol} \%])$. The mixture was heated to $95{ }^{\circ} \mathrm{C}$ for $1.5 \mathrm{~h}$ and then cooled to rt. (S)- azidomethyl phenylalanine $(100 \mathrm{mg}, 0.57 \mathrm{mmol})$ was added and the mixture allowed to stir for $12 \mathrm{~h}$. The mixture was concentrated and purified on silica gel eluting with $2: 1 \rightarrow$ 1:1 hexanes : EtOAc to give the furan as a clear oil $(210 \mathrm{mg}, 67 \%) .[\alpha]^{25} \mathrm{D}=-16.7\left(\mathrm{c}=0.43, \mathrm{CH}_{2} \mathrm{Cl}_{2}\right)$. ${ }^{1} \mathrm{H}$ NMR (400 MHz, $\left.\mathrm{CDCl}_{3}\right): \delta$ 7.31-7.19 (m, 5H); $4.87(\mathrm{~d}, J=2.4 \mathrm{~Hz}, 2 \mathrm{H}), 4.13(\mathrm{br} \mathrm{m}, 1 \mathrm{H}), 3.95$ (s, 3H); $3.82(\mathrm{~s}, 3 \mathrm{H}) ; 3.72-3.69(\mathrm{~m}, 2 \mathrm{H}) ; 3.45(\mathrm{dd}, J=4.4,12.4 \mathrm{~Hz}, 1 \mathrm{H}) ; 3.33(\mathrm{dd}, J=4.4,12.4 \mathrm{~Hz}$, $1 \mathrm{H}) ; 3.17-3.03(\mathrm{~m}, 2 \mathrm{H}) ; 2.88(\mathrm{dd}, J=6.8,13.6 \mathrm{~Hz}, 1 \mathrm{H}) ; 2.78(\mathrm{dd}, J=8,13.6 \mathrm{~Hz}, 1 \mathrm{H}) ; 2.53(\mathrm{t}, J=2.4$ $\mathrm{Hz}, 1 \mathrm{H}) ; 2.02-1.93$ (m, 1H); 1.90-1.81 (m, 1H); 1.14-0.96 (m, 21H). $\left.{ }^{13} \mathbf{C} \mathbf{~ N M R ~ ( 1 0 0 ~} \mathbf{~ M H z}, \mathbf{C D C l}_{3}\right)$ : $\delta 166.4,163.6,162.0,157.0,156.5,138.6,137.5,129.5,128.8,127.0,126.9,113.9,76.8,76.1,65.8$, 53.7, 53.3, 53.2, 52.4, 51.6, 51.2, 38.5, 30.3, 25.2, 18.2, 12.0. IR: 3307 (m); 2944 (m); 2101 (s); 1732, 1558 (both s). HRMS (ESI+): Calc. for $\mathrm{C}_{35} \mathrm{H}_{50} \mathrm{~N}_{5} \mathrm{O}_{9} \mathrm{Si}(\mathrm{m} / z)$ 712.3378; found $(\mathrm{m} / z) 712.3363$.

\section{2-allyl 3,4-dimethyl 5-((S)-3-(3-((R)-1-azido-3-phenylpropan-2-yl)ureido)-4-} (triisopropylsilyloxy)butyl)furan-2,3,4-tricarboxylate (4b).

Prepared according to general procedure C, with (S)-3a (0.39 $\mathrm{g}, 0.92 \mathrm{mmol})$ and $\mathrm{Rh}_{2} \mathrm{Oct}_{4}(7.2 \mathrm{mg}, 9.2 \mu \mathrm{mol})$. The isocyanate was trapped with (R)-azidomethylphenylalanine $(280 \mathrm{mg}$,

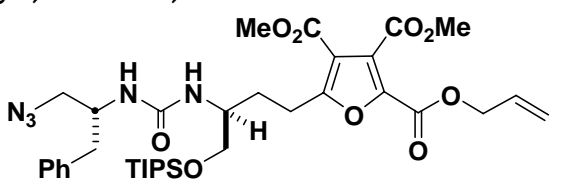
$1.60 \mathrm{mmol}$ ) and purified on silica gel eluting with 7:3 hexanes: EtOAc to give the furan as a yellow oil $(280 \mathrm{mg}, 43 \%)$. $[\alpha]^{25} \mathrm{D}=-33.4^{\circ}\left(\mathrm{c}=0.55, \mathrm{CH}_{2} \mathrm{Cl}_{2}\right) .{ }^{1} \mathbf{H}$ NMR $\left(500 \mathbf{~ M H z}, \mathrm{CDCl}_{3}\right): \delta$ 7.27-7.24 (m, 2H), 7.20-7.16 (m, 3H), $5.93(\mathrm{ddd}, J=6,11,16.5 \mathrm{~Hz}, 1 \mathrm{H}), 5.38(\mathrm{dd}, J=2.5,17.5 \mathrm{~Hz}$, $1 \mathrm{H}), 5.29$ (burried dd, $J=1,10 \mathrm{~Hz}, 1 \mathrm{H}), 4.76(\mathrm{~d}, J=6 \mathrm{~Hz}, 2 \mathrm{H}), 4.77-4.73$ (burried $\mathrm{m}, 1 \mathrm{H}), 4.68-$ 
$4.64(\mathrm{~m}, 1 \mathrm{H}), 4.15-4.11(\mathrm{~m}, 1 \mathrm{H}), 3.91(\mathrm{~s}, 3 \mathrm{H}), 3.81-3.80$ (burried $\mathrm{m}, 1 \mathrm{H}), 3.80(\mathrm{~s}, 3 \mathrm{H}), 3.70(\mathrm{dd}, J=$ 2.5, $10 \mathrm{~Hz}, 1 \mathrm{H}), 3.65(\mathrm{dd}, J=4.5,9.5 \mathrm{~Hz}, 1 \mathrm{H}), 3.41(\mathrm{dd}, J=4.5,12 \mathrm{~Hz}, 1 \mathrm{H}), 3.29(\mathrm{dd}, J=5,12.5$ $\mathrm{Hz}, 1 \mathrm{H}), 3.05(\mathrm{t}, J=7.5 \mathrm{~Hz}, 2 \mathrm{H}), 2.88(\mathrm{dd}, J=7.5,15 \mathrm{~Hz}, 1 \mathrm{H}), 2.78(\mathrm{dd}, J=8.5,13.5 \mathrm{~Hz}, 1 \mathrm{H})$,

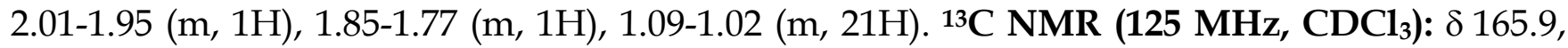
163.6, 161.9, 156.9, 156.8, 138.9, 137.3, 131.1 129.2, 128.5, 126.6, 126.0, 119.1, 113.5, 66.0, 65.6, 53.5, 52.9, 52.1, 51.4, 50.8, 38.2, 30.1, 24.9, 17.9, 11.8. IR (neat): 3360, 2945, 2865, 2101, 1735, 1730, 1653, 1636, 1604, 1560, 1555, 1448, 1288, 1223, 1165, 1118. HRMS (ESI+): Calc. for $\mathrm{C}_{35} \mathrm{H}_{51} \mathrm{~N}_{5} \mathrm{O}_{9} \mathrm{Si}$ $(\mathrm{m} / \mathrm{z}) 714.3534$. Found $(\mathrm{m} / \mathrm{z}) 714.3511$.

\section{2-allyl 3,4-dimethyl 5-((S)-3-(3-((S)-1-azido-3-phenylpropan-2-yl)ureido)-4-} (triisopropylsilyloxy)butyl)furan-2,3,4-tricarboxylate (4c). Prepared according to general procedure C, with (S)-3a (0.52 g, $1.23 \mathrm{mmol})$ and $\mathrm{Rh}_{2} \mathrm{Oct}_{4}(9.5 \mathrm{mg}, 12.3 \mu \mathrm{mol})$. The isocycanate was trapped with (S)-azidomethylphenylalanine $(280 \mathrm{mg}, 1.60$

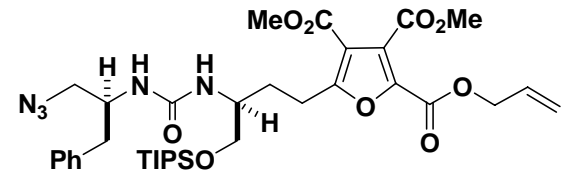
$\mathrm{mmol}$ ) and purified on silica eluting with 7:3 hexanes: EtOAc to give the furan as a yellow oil $(480 \mathrm{mg}, 55 \%) .[\alpha]^{25} \mathrm{D}=-32.8^{\circ} \quad\left(\mathrm{c}=0.78, \mathrm{CH}_{2} \mathrm{Cl}_{2}\right){ }^{1} \mathbf{H}$ NMR (400 MHz, CDCl $): \delta 7.30-7.18(\mathrm{~m}$, $5 \mathrm{H}), 5.94(\mathrm{ddd}, J=6,11.6,16.6 \mathrm{~Hz}, 1 \mathrm{H}), 5.39(\mathrm{dd}, J=2.5,22.5 \mathrm{~Hz}, 1 \mathrm{H}), 5.29(\mathrm{dd}, J=2.5,12.5 \mathrm{~Hz}$, $1 \mathrm{H}), 4.77(\mathrm{~d}, J=5.6 \mathrm{~Hz}, 2 \mathrm{H}), 4.73(\mathrm{~d}, J=8.8 \mathrm{~Hz}, 1 \mathrm{H}), 4.59-4.58(\mathrm{~m}, 1 \mathrm{H}), 4.14-4.09(\mathrm{~m}, 1 \mathrm{H}), 3.91(\mathrm{~s}$, $3 \mathrm{H}), 3.85-3.80$ (burried m, 1H), $3.80(\mathrm{~s}, 3 \mathrm{H}), 3.72-3.64(\mathrm{~m}, 2 \mathrm{H}), 3.44(\mathrm{dd}, J=4,12 \mathrm{~Hz}, 1 \mathrm{H}), 3.32$ $(\mathrm{dd}, J=4,12 \mathrm{~Hz}, 1 \mathrm{H}), 3.13-3.02(\mathrm{~m}, 2 \mathrm{H}), 2.87(\mathrm{dd}, J=6.4,13.6 \mathrm{~Hz}, 1 \mathrm{H}), 2.78(\mathrm{dd}, J=8,14 \mathrm{~Hz}$, 1H), 2.01-1.93 (m, 1H), 1.88-1.78 (m, 1H), 1.06-1.00 (m, 21H). ${ }^{13} \mathbf{C}$ NMR (125 MHz, $\left.\mathbf{C D C l}_{3}\right)$ : $\delta 165.8,163.6,161.9,156.8,138.9,137.3,131.1,129.2,128.6,126.6,126.0,119.1,113.5,66.0,65.6$, 53.4, 53.0, 52.1, 51.4, 50.9, 38.3, 30.1, 24.9, 17.9, 11.8. IR (neat): 3403, 2945, 2865, 2101, 1729, 1683, 1652, 1635, 1558, 1447, 1287, 1222, 1165, 1117. MS (APCI+) : Calc. for $\mathrm{C}_{35} \mathrm{H}_{51} \mathrm{~N}_{5} \mathrm{O} 9 \mathrm{Si}(\mathrm{m} / \mathrm{z})$ 713.34. Found $(m / z) 714.47$.

(S)-2-allyl 3,4-dimethyl 5-(3-(3-methyl-3-(prop-2-ynyl)ureido)-4(triisopropylsilyloxy)butyl)furan-2,3,4-tricarboxylate (5).

Prepared according to general procedure C, with (S)-3a (1.11 g, $2.60 \mathrm{mmol})$ and $\mathrm{Rh}_{2} \mathrm{Oct}_{4}(20 \mathrm{mg}, 26 \mu \mathrm{mol})$. The isocycanate was

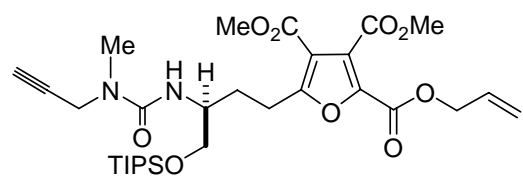
trapped with $N$-methylpropargylamine $(282 \mu \mathrm{L}, 3.39 \mathrm{mmol})$ and purified on silica eluting with 3:1 hexanes: EtOAc to give the furan as a yellow oil $(1.12 \mathrm{~g}, 73 \%)$. $[\alpha]^{25} \mathrm{D}=-18.4^{\circ}(\mathrm{c}=0.34$, $\mathrm{CH}_{2} \mathrm{Cl}_{2}$ ). ${ }^{1} \mathrm{H}$ NMR (400 MHz, $\left.\mathrm{CDCl}_{3}\right): \delta 5.94(\mathrm{ddd}, J=6,11,17 \mathrm{~Hz}, 1 \mathrm{H}) ; 5.38(\mathrm{~d}, J=17 \mathrm{~Hz}, 1 \mathrm{H})$; $5.24(\mathrm{~d}, J=11 \mathrm{~Hz}, 1 \mathrm{H}) ; 4.96(\mathrm{~d}, J=8.4 \mathrm{~Hz}, 1 \mathrm{H}) ; 4.76(\mathrm{~d}, J=6 \mathrm{~Hz}, 1 \mathrm{H}) ; 4.15(1 / 2 \mathrm{ABdd}, J=2.4,18$ $\mathrm{Hz}, 1 \mathrm{H}) ; 4.08(1 / 2 \mathrm{ABdd}, J=2.4,18 \mathrm{~Hz}, 1 \mathrm{H}) ; 3.96-3.90$ (burried m, 1H); 3.91 (s, 3H); 3.81 (s, $3 \mathrm{H}) ; 3.75(\mathrm{~d}, J=3.2 \mathrm{~Hz}, 2 \mathrm{H}) ; 3.10(\mathrm{ddd}, J=6.4,9.2,14.4,2 \mathrm{H}) ; 2.93(\mathrm{~s}, 3 \mathrm{H}) ; 2.22(\mathrm{t}, J=2 \mathrm{~Hz}, 1 \mathrm{H})$; 2.20-1.90 (m, 2H); 1.10-1.00 (m, 21H). $\left.{ }^{13} \mathbf{C ~ N M R ~ ( 1 0 0 ~ M H z , ~} \mathbf{C D C l}_{3}\right): \delta 166.2 ; 163.9,162.1 ; 157.5$; $157.0 ; 139.1 ; 131.4 ; 126.4 ; 119.4 ; 113.7 ; 79.5 ; 72.2 ; 66.2 ; 65.6 ; 53.2 ; 52.3 ; 51.9 ; 37.9 ; 33.8 ; 30.6 ; 25.4$, 18.2, 12.0. IR (Dep $\mathbf{C D C l}_{3}$ ): 3308 (w); 2944, 2865 (both m); 1728 (s); 1651, 1524, 1222 (all m). HRMS (ESI+): Calc. for $\mathrm{C}_{29} \mathrm{H}_{45} \mathrm{~N}_{2} \mathrm{O} 9 \mathrm{Si}(\mathrm{m} / z) 607.3051$; found $(\mathrm{m} / z) 607.3069$.

2-allyl 3,4-dimethyl 5-((R)-3-(3-((S)-1-phenylbut-3-yn-2yl)ureido)-4-(triisopropylsilyloxy)butyl)furan-2,3,4-tricarboxylate and 2-allyl 3,4-dimethyl 5-((S)-3-(3-((R)-1-phenylbut-3-yn-2yl)ureido)-4-(triisopropylsilyloxy)butyl)furan-2,3,4-tricarboxylate $(6 \mathrm{a}(5 S, 9 R)$ and $6 \mathrm{~d}(5 R, 9 S))$. 6a was prepared according to General

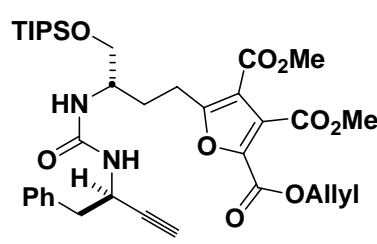
Procedure C with (S)-3a (487 mg, $1.15 \mathrm{mmol})$ and $\mathrm{Rh}_{2} \mathrm{Oct}_{4}(9 \mathrm{mg}, 12 \mu \mathrm{mol})$, trapping the 
isocyanante with $(R)$-2-aminophenyl butyne $(200 \mathrm{mg}, 1.38 \mathrm{mmol})$ and purified on silica eluting with 3:1 Hexanes: EtOAc to give the urea as a clear oil $(571 \mathrm{mg}, 67 \%)$. $6 \mathbf{b}$ was prepared according to general procedure $C$ with $(R)-3 a(487 \mathrm{mg}, 1.15 \mathrm{mmol})$ and $\mathrm{Rh}_{2} \mathrm{Oct}_{4}(9 \mathrm{mg}, 12$ $\mu \mathrm{mol})$, trapping the isocyanante with $S$-2-aminophenyl butyne $(200 \mathrm{mg}, 1.38 \mathrm{mmol}$ ) and purified on silica eluting with 3:1 hexanes: EtOAc to give the urea as a clear oil $(670 \mathrm{mg}, 79 \%)$. $(6 \mathrm{a} ; 5 \mathrm{~S}, 9 \mathrm{R})[\alpha]^{25} \mathrm{D}=-11.36\left(\mathrm{c}=0.44, \mathrm{CH}_{2} \mathrm{Cl}_{2}\right) ;(6 \mathrm{~d} ; 5 \mathrm{R}, 9 \mathrm{~S})[\alpha]^{25} \mathrm{D}=+3.48\left(\mathrm{c}=0.5, \mathrm{CH}_{2} \mathrm{Cl}_{2}\right) .{ }^{1} \mathrm{H}$ NMR (400 MHz, CDCl $): \delta 7.28-7.19(\mathrm{~m}, 5 \mathrm{H}) ; 5.94(\mathrm{ddd}, J=6,10,17 \mathrm{~Hz}, 1 \mathrm{H}) ; 5.38(\mathrm{ddd}, J=1.6$, 1.6, $17 \mathrm{~Hz}, 1 \mathrm{H}) ; 5.28(\mathrm{ddd}, J=1.6,1.6,10 \mathrm{~Hz}, 1 \mathrm{H}) ; 4.84-4.78$ (buried $\mathrm{m}, 1 \mathrm{H}) ; 4.77(\mathrm{dt}, J=1.2,5.6$ $\mathrm{Hz}, 2 \mathrm{H}) ; 4.68($ br d, $J=8 \mathrm{~Hz}, 1 \mathrm{H}) ; 3.91(\mathrm{~s}, 3 \mathrm{H}) ; 3.87-3.83$ (buried m, 1H); $3.80(\mathrm{~s}, 3 \mathrm{H}) ; 3.72(\mathrm{dd}, J$ $=3.2,10 \mathrm{~Hz}, 1 \mathrm{H}) ; 3.67(\mathrm{dd}, J=4.8,10 \mathrm{~Hz}, 1 \mathrm{H}) ; 3.08(\mathrm{app} \mathrm{t}, J=8 \mathrm{~Hz}, 2 \mathrm{H}) ; 3.01(\mathrm{dd}, J=5.2,13.2$ $\mathrm{Hz}, 1 \mathrm{H}) ; 2.94(\mathrm{dd}, J=7.2,13.2 \mathrm{~Hz}, 1 \mathrm{H}) ; 2.80(\mathrm{~d}, J=2.4 \mathrm{~Hz}, 1 \mathrm{H}) ; 2.04-1.95(\mathrm{~m}, 1 \mathrm{H}) ; 1.89-1.79(\mathrm{~m}$, 1H); 1.14-1.01 (m, $21 \mathrm{H}) .{ }^{13} \mathbf{C}$ NMR (100 MHz, $\left.\mathbf{C D C l}_{3}\right)$ : $\delta$ 166.0, 163.8, 162.0, 157.0, 156.7, 139.2, 136.7, 131.3, 129.9, 128.4, 127.0, 126.3, 119.3, 113.7, 83.4, 72.3, 66.2, 65.8, 53.2, 52.3, 51.7, 43.5, 41.9, 30.4, 25.2, 18.1, 12.0. IR (Dep. $\mathrm{CDCl}_{3}$ ): 3306, 2943, 2864 (all w), 1724, 1552, 1219 (all s). HRMS (ESI+): Calc. for $\mathrm{C}_{36} \mathrm{H}_{51} \mathrm{~N}_{2} \mathrm{O}_{9} \mathrm{Si}(\mathrm{m} / \mathrm{z})$ 683.3364; found $(\mathrm{m} / \mathrm{z}) 683.3342$.

\section{2-allyl 3,4-dimethyl 5-((R)-3-(3-((R)-1-phenylbut-3-yn-2-} yl)ureido)-4-(triisopropylsilyloxy)butyl)furan-2,3,4-tricarboxylate and 2-allyl 3,4-dimethyl 5-((S)-3-(3-((S)-1-phenylbut-3-yn-2yl)ureido)-4-(triisopropylsilyloxy)butyl)furan-2,3,4-tricarboxylate $(6 \mathrm{~b}(5 S, 9 S), 6 \mathrm{c}(5 R, 9 R))$. $6 \mathrm{~b}$ was prepared according to general

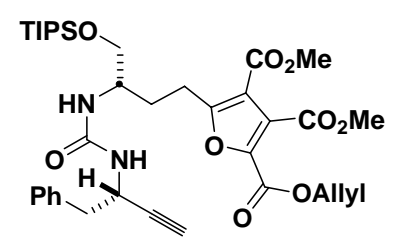
procedure C (S)-3a (487 mg, $1.15 \mathrm{mmol})$ and $\mathrm{Rh}_{2} \mathrm{Oct}_{4}(9 \mathrm{mg}, 12 \mu \mathrm{mol})$, trapping the isocyanante with (S)-2-aminophenyl butyne $(200 \mathrm{mg}, 1.38 \mathrm{mmol})$ and purified on silica eluting with 3:1 Hexanes: EtOAc to give the urea as a clear oil $(590 \mathrm{mg}, 69 \%) .6 \mathrm{c}$ was prepared according to General Procedure C (R)-3a (487 mg, $1.15 \mathrm{mmol})$ and $\mathrm{Rh}_{2} \mathrm{Oct}_{4}(9 \mathrm{mg}, 12 \mu \mathrm{mol})$, trapping the isocyanante with (R)-2-aminophenyl butyne $(200 \mathrm{mg}, 1.38 \mathrm{mmol})$ and purified on silica eluting with 3:1 Hexanes: Ethyl acetate to give the urea as a clear oil $(591 \mathrm{mg}, 69 \%) .(6 \mathrm{~b} ; 5 S, 9 S)[\alpha]{ }^{25} \mathrm{D}$ $=-5.52^{\circ}\left(\mathrm{c}=0.58, \mathrm{CH}_{2} \mathrm{Cl}_{2}\right) ;(6 \mathrm{c} ; 5 R, 9 R)[\alpha]^{25} \mathrm{D}=+7.00^{\circ}\left(\mathrm{c}=0.5, \mathrm{CH}_{2} \mathrm{Cl}_{2}\right) .{ }^{1} \mathbf{H} \mathbf{~ N M R}(400 \mathrm{MHz}$, $\left.\mathrm{CDCl}_{3}\right): \delta 7.32-7.21(\mathrm{~m}, 5 \mathrm{H}) ; 5.94(\mathrm{ddd}, J=6,10,17 \mathrm{~Hz}, 1 \mathrm{H}) ; 5.36(\mathrm{ddd}, J=1.6,1.6,17 \mathrm{~Hz}, 1 \mathrm{H})$; $5.28(\mathrm{ddd}, J=1.6,1.6,10 \mathrm{~Hz}, 1 \mathrm{H}) ; 4.86-4.80(\mathrm{~m}, 1 \mathrm{H}) ; 4.75(\mathrm{dt}, J=1.6,6 \mathrm{~Hz}, 2 \mathrm{H}) ; 4.68(\mathrm{br} \mathrm{d}, J=$ $8 \mathrm{~Hz}, 1 \mathrm{H}) ; 3.91(\mathrm{~s}, 3 \mathrm{H}) ; 3.87-3.80$ (buried $\mathrm{m}, 1 \mathrm{H}) ; 3.79(\mathrm{~s}, 3 \mathrm{H}) ; 3.70(\mathrm{~d}, J=3.7 \mathrm{~Hz}, 2 \mathrm{H}) ; 3.09$ (dd, $J$ $=5.6,8 \mathrm{~Hz}, 2 \mathrm{H}) ; 3.00(\mathrm{dd}, J=5.2,13.2 \mathrm{~Hz}, 1 \mathrm{H}) ; 2.91(\mathrm{dd}, J=7.2,13.2 \mathrm{~Hz}, 1 \mathrm{H}) ; 2.26(\mathrm{~d}, J=2 \mathrm{~Hz}$, 1H); 2.00-1.92 (m, 1H); 1.88-1.79 (m, 1H); 1.14-1.01 (m, $21 \mathrm{H}) .{ }^{13} \mathbf{C}$ NMR (100 MHz, $\left.\mathbf{C D C l}_{3}\right)$ : $\delta 165.8,163.7,161.9,157.0,156.7,139.1,136.7,131.3,129.9,128.3,126.9,126.3,119.3,113.8,83.4$,

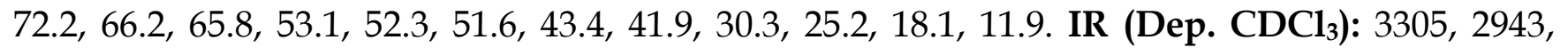
2864 (all w), 1724, 1552, 1219 (all s). HRMS (ESI+): Calc. for $\mathrm{C}_{36} \mathrm{H}_{51} \mathrm{~N}_{2} \mathrm{O} 9 \mathrm{Si}(\mathrm{m} / \mathrm{z})$ 683.3364; found $(\mathrm{m} / \mathrm{z}) 683.3336$.

2-allyl 3,4-dimethyl 5-((S)-3-(3-((R)-1-oxo-3-phenyl-1-(prop-2-ynylamino)propan-2-yl)ureido)-4(triisopropylsilyloxy)butyl)furan-2,3,4-tricarboxylate (7a $(5 S, 9 R), 7 \mathrm{~d}(5 R$, 9S)). 7a was prepared according to General Procedure C, with (S)-3a (796 mg, $1.88 \mathrm{mmol}$ ) and $\mathrm{Rh}_{2} \mathrm{Oct}_{4}(15 \mathrm{mg}, 19 \mu \mathrm{mol})$, trapping the isocyanante with (S)-phenylalanine propargylamide (392 mg, 1.65 mmol,) and purified on silica eluting with 1:1 Hexanes: EtOAc to give the urea as a clear oil $723 \mathrm{mg}(71 \%)$. $\mathbf{7 d}$ was prepared according to General Procedure C, with (R)-3a (796 mg, $1.88 \mathrm{mmol})$ and $\mathrm{Rh}_{2} \mathrm{Oct}_{4}(15$

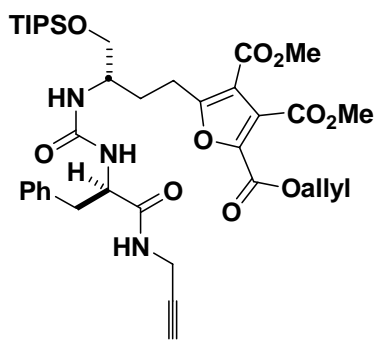


$\mathrm{mg}, 19 \mu \mathrm{mol})$, trapping the isocyanante with $(R)$-phenylalanine propargylamide (392 $\mathrm{mg}, 1.65$ mmol,) and purified on silica eluting with 1:1 Hexanes: EtOAc to give the urea as a clear oil $840 \mathrm{mg}(83 \%) .(7 \mathrm{a} ; 5 S, 9 R)[\alpha]^{25} \mathrm{D}=+4.2\left(\mathrm{c}=0.60, \mathrm{CH}_{2} \mathrm{Cl}_{2}\right) ;(7 \mathrm{~d} ; 5 R, 9 S)[\alpha]^{25} \mathrm{D}=-1.8(\mathrm{c}=0.47$, $\mathrm{CH}_{2} \mathrm{Cl}_{2}$ ). ${ }^{1} \mathrm{HNMR}\left(400 \mathrm{MHz}, \mathrm{CDCl}_{3}\right): \delta 7.26-7.13(\mathrm{~m}, 5 \mathrm{H}) ; 6.65(t, J=6.8 \mathrm{~Hz}, 1 \mathrm{H}) ; 5.92$ (dddd, $J$ $=5.6,5.6,10.4,17.2 \mathrm{~Hz}, 1 \mathrm{H}) ; 5.35(\mathrm{ddd}, J=2,3.2,17.2 \mathrm{~Hz}, 1 \mathrm{H}) ; 5.27(\mathrm{ddd}, J=1.2,1.2,10.4 \mathrm{~Hz}$, $1 \mathrm{H}) ; 5.27(\mathrm{~d}, J=8.0 \mathrm{~Hz}, 1 \mathrm{H}) ; 4.98(\mathrm{~d}, J=8.8 \mathrm{~Hz}, 1 \mathrm{H}) ; 4.76(\mathrm{dt}, J=1.6,6 \mathrm{~Hz}, 2 \mathrm{H}) ; 4.59(\mathrm{q}, J=6.8$ $\mathrm{Hz}, 1 \mathrm{H}) ; 3.92(\mathrm{dd}, J=2.4,5.6 \mathrm{~Hz}, 2 \mathrm{H}) ; 3.91(\mathrm{~s}, 3 \mathrm{H}) ; 3.78(\mathrm{~s}, 3 \mathrm{H}) ; 3.70(\mathrm{dd}, J=3.2,10.4 \mathrm{~Hz}, 1 \mathrm{H})$, $3.59(\mathrm{dd}, J=5.6,10 \mathrm{~Hz}, 1 \mathrm{H}) ; 3.11-2.95(\mathrm{~m}, 4 \mathrm{H}) ; 2.18(\mathrm{t}, J=2.4 \mathrm{~Hz}, 1 \mathrm{H}) ; 2.04-1.94(\mathrm{~m}, 1 \mathrm{H}) ; 1.83-$ $1.74(\mathrm{~m}, 1 \mathrm{H}) ; 1.03-0.98(\mathrm{~m}, 21 \mathrm{H}) .{ }^{\mathbf{1 3}} \mathbf{C}$ NMR (100 MHz, $\left.\mathbf{C D C l}_{3}\right): \delta 172.0,166.1,163.8,162.1,157.4$, 157.1, 139.2, 137.0, 131.3, 129.5, 128.8, 127.0, 126.3, 119.4, 113.8, 79.3, 71.7, 66.3, 65.77, 55.4, 53.2, 52.4, 51.8, 38.8, 30.3, 29.3, 25.1, 18.2, 12.0. IR (Dep. CDCl $_{3}$ ): 3270, 2944, 2865 (all w); 1725, 1631, 1552, 1220 (all s). HRMS (ESI+): Calc. for [M+Na] $\mathrm{C}_{38} \mathrm{H}_{54} \mathrm{~N}_{3} \mathrm{O}_{10} \mathrm{SiNa}(\mathrm{m} / \mathrm{z}) 762.3398$; found $(\mathrm{m} / \mathrm{z}) 762.3401$.

2-allyl 3,4-dimethyl 5-((S)-3-(3-((S)-1-oxo-3-phenyl-1-(prop-2-ynylamino)propan-2-yl)ureido)-4(triisopropylsilyloxy)butyl)furan-2,3,4-tricarboxylate (7b (5S, 9S), 7c TIPSO

$(5 R, 9 R)) .7 b$ was prepared according to General Procedure $C,(S)-3 a$ (796 mg, $1.88 \mathrm{mmol})$ and $\mathrm{Rh}_{2} \mathrm{Oct}_{4}(15 \mathrm{mg}, 19 \mu \mathrm{mol})$, trapping the isocyanante with (S)-phenylalanine propargylamide (392 mg, 1.65 mmol,) and purified on silica eluting with 1:1 Hexanes: EtOAc to give the urea as a light yellow foam; $615 \mathrm{mg}(60 \%)$. $7 \mathrm{c}$ was prepared according to General Procedure C, with (R)-3a (796 mg, $1.88 \mathrm{mmol})$

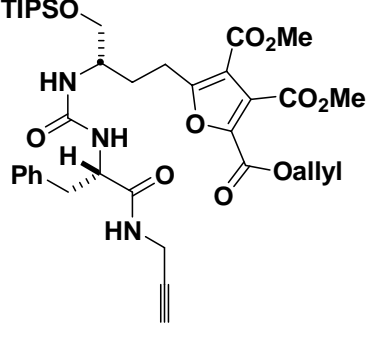
and $\mathrm{Rh}_{2} \mathrm{Oct}_{4}(15 \mathrm{mg}, 19 \mu \mathrm{mol})$, trapping the isocyanante with (R)-phenylalanine propargylamide (392 mg, $1.65 \mathrm{mmol}$ ) ) and purified on silica eluting with 1:1 Hexanes: EtOAc to give the urea as a light yellow foam; $612 \mathrm{mg}(60 \%) . \quad(7 \mathrm{~b} ; 5 S, 9 S)[\alpha]^{25} \mathrm{D}=-8.0 \quad(\mathrm{c}=0.52$, $\left.\mathrm{CH}_{2} \mathrm{Cl}_{2}\right) ;(7 \mathrm{c} ; 5 R, 9 R)\left[\alpha{ }^{25} \mathrm{D}=+7.9\left(\mathrm{c}=0.40, \mathrm{CH}_{2} \mathrm{Cl}_{2}\right) .{ }^{1} \mathbf{H}\right.$ NMR $\left(400 \mathrm{MHz}, \mathrm{CDCl}_{3}\right): \delta$ 7.26-7.17 $(\mathrm{m}, 5 \mathrm{H}) ; 6.92(t, J=5.6 \mathrm{~Hz}, 1 \mathrm{H}) ; 5.92(\mathrm{dddd}, J=5.6,5.6,10.4,17.2 \mathrm{~Hz}, 1 \mathrm{H}) ; 5.42(\mathrm{~d}, J=8 \mathrm{~Hz}, 1 \mathrm{H})$; $5.36(\mathrm{ddd}, J=1.2,1.2,17.2 \mathrm{~Hz}, 1 \mathrm{H}) ; 5.27(\mathrm{ddd}, J=1.2,1.2,10.4 \mathrm{~Hz}, 1 \mathrm{H}) ; 5.07(\mathrm{~d}, J=8.8 \mathrm{~Hz}, 1 \mathrm{H})$; $4.74(\mathrm{dt}, J=1.6,6 \mathrm{~Hz}, 2 \mathrm{H}) ; 4.59(\mathrm{q}, J=6.8 \mathrm{~Hz}, 1 \mathrm{H}) ; 3.92(\mathrm{ddd}, J=2.4,6.4,8 \mathrm{~Hz}, 2 \mathrm{H}) ; 3.89(\mathrm{~s}, 3 \mathrm{H})$; 3.78-3.75 (buried m, 1H); $3.77(\mathrm{~s}, 3 \mathrm{H}) ; 3.08-3.00(\mathrm{~m}, 4 \mathrm{H}) ; 2.14(\mathrm{t}, J=2.4 \mathrm{~Hz}, 1 \mathrm{H}) ; 2.03-1.94(\mathrm{~m}$, 1H); 1.80-1.71 (m, 1H); 1.05-0.98 (m, 21H). ${ }^{13} \mathbf{C}$ NMR (100 MHz, $\left.\mathbf{C D C l}_{3}\right): \delta 172.2,165.9,163.8$, 162.1, 157.4, 157.1, 139.2, 137.1, 131.3, 129.6, 128.7, 126.9, 126.2, 119.4, 113.7, 79.4, 71.6, 66.3, 65.9, 55.2, 53.2, 52.4, 51.6, 38.8, 30.4, 29.2, 25.1, 18.2, 12.0. IR (Dep. CDCl $): 3270,2943$, 2865 (all w); 1725, 1630, 1551, 1220, 907 (all s). HRMS (ESI+): Calc. for [M+Na] $\mathrm{C}_{38} \mathrm{H}_{54} \mathrm{~N}_{3} \mathrm{O}_{10} \mathrm{SiNa}(\mathrm{m} / \mathrm{z}$ ) 762.3398; found $(\mathrm{m} / \mathrm{z}) 762.3419$.

(S)-allyl 5-(3-(3-methyl-3-(prop-2-ynyl)ureido)-4-(triisopropylsilyloxy)butyl)-4-tosylfuran-2carboxylate (8). Prepared according to general procedure $\mathrm{C}$, with (S)-3a (32 mg, $76 \mu \mathrm{mol}), p$-tolylethynyl sulfone (14 mg, 76 $\mu \mathrm{mol})$, and $\mathrm{Rh}_{2} \mathrm{Oct}_{4}(1.0 \mathrm{mg}, 1 \mu \mathrm{mol})$. The isocycanate was trapped with $\mathrm{N}$-methylpropargylamine $(19 \mu \mathrm{L}, 0.23 \mathrm{mmol})$ and purified by PTLC eluting with 3:1 hexanes: EtOAc to give the

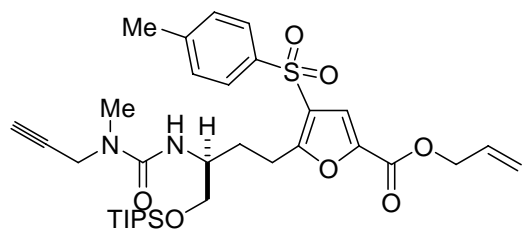
furan as a clear oil $(37 \mathrm{mg}, 76 \%) .[\alpha]{ }^{25} \mathrm{D}=-24.2\left(\mathrm{c}=0.08, \mathrm{CH}_{2} \mathrm{Cl}_{2}\right) .{ }^{1} \mathbf{H}$ NMR $(400 \mathrm{MHz}$, $\left.\mathrm{CDCl}_{3}\right): \delta 7.78(\mathrm{~d}, J=8 \mathrm{~Hz}, 2 \mathrm{H}) ; 7.31(\mathrm{~d}, J=8 \mathrm{~Hz}, 2 \mathrm{H}) ; 7.26(\mathrm{~s}, 1 \mathrm{H}) ; 5.94(\mathrm{ddt}, J=5.6,10.4,18 \mathrm{~Hz}$, $1 \mathrm{H}) ; 5.36(\mathrm{ddd}, J=2,2,18 \mathrm{~Hz}, 1 \mathrm{H}), 5.28(\mathrm{ddd}, J=2,2,10.4 \mathrm{~Hz}, 1 \mathrm{H}) ; 5.02(\mathrm{~d}, J=8.4 \mathrm{~Hz}, 1 \mathrm{H}) ; 4.74$ $(\mathrm{dt}, J=5.6,1 \mathrm{~Hz}, 2 \mathrm{H}) ; 4.15(\mathrm{~d}, J=2.4 \mathrm{~Hz}, 2 \mathrm{H}) ; 3.97-3.91(\mathrm{~m}, 1 \mathrm{H}) ; 3.76(\mathrm{dd}, J=4.4,10 \mathrm{~Hz}, 1 \mathrm{H})$; 
$4.73(\mathrm{dd}, J=2.8,10 \mathrm{~Hz}, 1 \mathrm{H}) ; 3.16(\mathrm{ddd}, J=4.8,10.8,14.4 \mathrm{~Hz}, 1 \mathrm{H}) ; 3.06-2.95(\mathrm{~m}, 1 \mathrm{H}) ; 2.96(\mathrm{~s}, 3 \mathrm{H})$; $2.41(\mathrm{~s}, 3 \mathrm{H}) ; 2.23(\mathrm{t}, J=2.4 \mathrm{~Hz}, 1 \mathrm{H}) ; 2.05-1.91(\mathrm{~m}, 2 \mathrm{H}) ; 1.08-1.02(\mathrm{~m}, 21 \mathrm{H}) .{ }^{13} \mathrm{C} \mathbf{N M R}(\mathbf{1 0 0} \mathbf{M H z}$, $\left.\mathrm{CDCl}_{3}\right): \delta 163.4,157.6,157.5,144.9,143.1,138.9,131.5,130.3,127.3,125.3,119.4,117.6,79.5,72.2$, 66.1, 65.7, 51.9, 37.9, 33.8, 31.2, 24.8, 21.8, 18.2, 12.0. IR (Dep. CDCl 3 ): 3426, 3307 (br, w), 2842, 2865, 1737, 1657, 1530, 1170, 1155 (all s). NOE (500 MHz, CD 3 CN): : Irradiation 3.13-3.07 ppm; enhancements: 7.4 (1 \%); 5.1 (1\%); 3.82 (2\%); 2.05 (3\%); 1.85 (3\%). HRMS (ESI+): Calc. for $\mathrm{C}_{33} \mathrm{H}_{49} \mathrm{~N}_{2} \mathrm{O}_{7} \mathrm{Si}(\mathrm{m} / z) 645.3030$. Found $(\mathrm{m} / z) 645.3030$.

(S)-2-allyl 4-methyl 5-(3-(3-methyl-3-(prop-2-ynyl)ureido)-4(triisopropylsilyloxy)butyl)oxazole-2,4-dicarboxylate (9).

Prepared according to general procedure C, with (S)-3a (98 mg, $0.23 \mathrm{mmol})$, methyl cyanoformate $(55 \mu \mathrm{L}, 0.69 \mathrm{mmol})$, and $\mathrm{Rh}_{2} \mathrm{Oct}_{4}(9 \mathrm{mg}, 11 \mu \mathrm{mol})$. The isocycanate was trapped with $N$ -

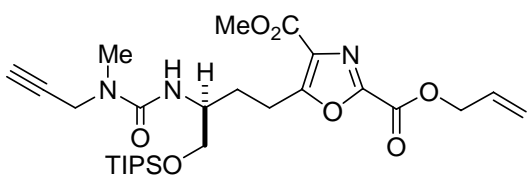
methylpropargylamine $(57 \mu \mathrm{L}, 0.69 \mathrm{mmol})$ and purified on silica gel eluting with 3:1 hexanes: EtOAc to give the oxazole as a clear oil $(63 \mathrm{mg}, 50 \%)$. $[\alpha]^{25} \mathrm{D}=-9.31$ (c = 0.10, $\left.\mathrm{CH}_{2} \mathrm{Cl}_{2}\right) .{ }^{1} \mathbf{H}$ NMR (400 MHz, $\left.\mathrm{CDCl}_{3}\right): \delta 5.98(\mathrm{ddt}, J=5.6,10.4,18 \mathrm{~Hz}, 1 \mathrm{H}) ; 5.42(\mathrm{ddd}, J=2,2,18 \mathrm{~Hz}, 1 \mathrm{H})$, $5.25(\mathrm{ddd}, J=2,2,10.4 \mathrm{~Hz}, 1 \mathrm{H}) ; 4.97(\mathrm{~d}, J=8.4 \mathrm{~Hz}, 1 \mathrm{H}) ; 4.82(\operatorname{app~dt}, J=2,5.6 \mathrm{~Hz}, 2 \mathrm{H}) ; 4.10$ (dd, $J=2.4,17.6 \mathrm{~Hz}, 1 \mathrm{H}) ; 4.04(\mathrm{dd}, J=2.4,17.6 \mathrm{~Hz}, 1 \mathrm{H}) ; 4.0-3.94(\mathrm{~m}, 1 \mathrm{H}) ; 3.92(\mathrm{~s}, 3 \mathrm{H}) ; 3.78-3.72(\mathrm{~m}$, $2 \mathrm{H}) ; 2.95(\mathrm{dd}, J=7.2,8.8 \mathrm{~Hz}, 1 \mathrm{H}) ; 2.89(\mathrm{~s}, 3 \mathrm{H}) ; 2.21(\mathrm{t}, J=2.4 \mathrm{~Hz}, 1 \mathrm{H}) ; 2.12-2.07(\mathrm{~m}, 2 \mathrm{H}) ; 1.14-$ $1.00(\mathrm{~m}, 21 \mathrm{H}) .{ }^{13} \mathrm{C}$ NMR (100 MHz, $\left.\mathbf{C D C l}_{3}\right): \delta 166.8,161.0,157.4,156.7,142.4,136.2,131.2$, 119.7, 79.3, 72.2, 66.7, 65.6, 53.0, 51.7, 37.9, 33.8, 29.6, 25.6, 18.2, 12.0. IR (Dep. CDCl 3 ): 3309 (br), 2943, 2865, 1734, 1635, 1528, 1153 (all s). HRMS (ESI+): Calc. for $\mathrm{C}_{27} \mathrm{H}_{44} \mathrm{~N}_{3} \mathrm{O}_{7} \mathrm{Si}(\mathrm{m} / z)$ 550.2948. Found $(m / z) 550.2946$.

$V$ General procedure $D$ for the deprotection and coupling of the allyl ester:

Linear Precursor to 12a. To a solution of the allyl ester $(0.10 \mathrm{~g}, 0.15$ $\mathrm{mmol})$ in THF $(5 \mathrm{~mL})$ was added $N$ - methylmorpholine $(133 \mu \mathrm{L}, 1.5$ mmol, 10 equiv.) and $\mathrm{Pd}\left(\mathrm{PPh}_{3}\right)_{4}(1.7 \mathrm{mg}, 1.5 \mu \mathrm{mol}, 1 \mathrm{~mol} \%)$. The mixture was stirred until the reaction was complete by TLC $(\sim 0.5 \mathrm{~h})$. The mixture was concentrated, taken up in EtOAc and washed with $10 \% \mathrm{HCl}$. The organics were concentrated and taken up in 3:1

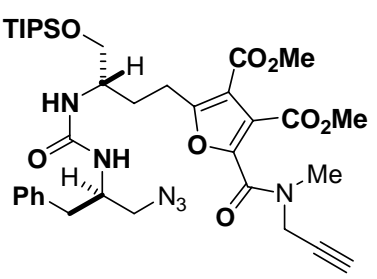
$\mathrm{CH}_{2} \mathrm{Cl}_{2}$ :DMF. $N$-Methylpropargylamine $(33 \mu \mathrm{L}, 0.37 \mathrm{mmol}), \quad N, N$-Diisopropylethylamine $(0.1$ $\mathrm{mL}, 0.56 \mathrm{mmol})$ and PyBOP $(0.1 \mathrm{~g}, 0.18 \mathrm{mmol})$ were added and the solution was stirred at r.t for $12 \mathrm{~h}$. The reaction mixture was then diluted with EtOAc and washed with $10 \% \mathrm{HCl}$, saturated aqueous $\mathrm{NaHCO}_{3}$ and brine. The organic layer was dried over $\mathrm{Na}_{2} \mathrm{SO}_{4}$ and the solvent was removed under reduced pressure. The resulting residue was purified on silica eluting with 1:1 hexanes : EtOAc to give the amide as a yellow oil $(83 \mathrm{mg}, 71 \%) .[\alpha]{ }^{25} \mathrm{D}=-10.7$ (c = 0.11, $\left.\mathrm{CH}_{2} \mathrm{Cl}_{2}\right)$. ${ }^{1} \mathbf{H}$ NMR (500 $\left.\mathbf{~ M H z}, \mathbf{C D C l}_{3}\right)$ : 7.28-7.25 (m, 2H), 7.20-7.17 (m, 3H), $4.73(\mathrm{~d}, J=$ $8.5 \mathrm{~Hz}, 1 \mathrm{H}), 4.70(\mathrm{~d}, J=7.5 \mathrm{~Hz}, 1 \mathrm{H}), 4.30(\mathrm{~d}, J=2.5 \mathrm{~Hz}, 2 \mathrm{H}), 4.13-4.09(\mathrm{~m}, 1 \mathrm{H}), 3.88(\mathrm{~s}, 3 \mathrm{H}), 3.84-$ 3.78 (burried m, 1H), $3.3 .80(\mathrm{~s}, 3 \mathrm{H}), 3.73(\mathrm{dd}, J=3.5,9.5 \mathrm{~Hz}, 1 \mathrm{H}), 3.65(\mathrm{dd}, J=5,10 \mathrm{~Hz}, 1 \mathrm{H}), 3.40$ $(\mathrm{dd}, J=4,12 \mathrm{~Hz}, 1 \mathrm{H}), 3.27(\mathrm{dd}, J=5,12.5 \mathrm{~Hz}, 1 \mathrm{H}), 3.19$ (br s, 3H), 3.08 (ddd, J = 5, 10, $15 \mathrm{~Hz}$, $1 \mathrm{H}), 2.97(\mathrm{ddd}, J=5,10,15 \mathrm{~Hz}, 1 \mathrm{H}), 2.87(\mathrm{dd}, J=7.5,15 \mathrm{~Hz}, 1 \mathrm{H}), 2.75(\mathrm{dd}, J=7.5,12.5 \mathrm{~Hz}, 1 \mathrm{H})$, 2.28 (br s, 1H), 2.01-1.94 (m, 1H), 1.85 (ddd, $J=10,17.5,20 \mathrm{~Hz}, 1 \mathrm{H}), 1.03-1.02(\mathrm{~m}, 21 \mathrm{H}) .{ }^{13} \mathrm{C}$ NMR (125 MHz, CDCl $)$ : 163.5, 163.2, 162.3, 158.3, 156.8, 144.0, 137.4, 129.2, 128.5, 126.6, 123.8, 113.20, 77.7, 72.8, 65.4, 53.4, 52.8, 52.0, 51.2, 50.9, 38.2, 29.9, 24.6, 17.9, 11.8. IR (neat): 3377, 3309, 
2944, 2865, 2100, 1726, 1636, 1603, 1558, 1506, 1496, 1448, 1406, 1285, 1223, 1113, 1044. Found $(\mathrm{m} / \mathrm{z})$ 725.73. HRMS (ESI+): Calc. for $\mathrm{C}_{36} \mathrm{H}_{52} \mathrm{~N}_{6} \mathrm{O}_{8} \mathrm{Si}(\mathrm{m} / \mathrm{z})$ 725.3694. Found $(\mathrm{m} / \mathrm{z}) 725.3678$.

Linear Precursor to $\mathbf{1 2 b}$. The propargyl amide was prepared according to General Procedure D, with the ester $4 \mathrm{c}(0.38 \mathrm{~g}, 0.56 \mathrm{mmol})$. The crude organics were purified on silica eluting with 1:1 hexanes:EtOAc to give the amide as a white solid $(265 \mathrm{mg}, 70 \%) .[\alpha]^{25} \mathrm{D}=-3.2(\mathrm{c}=0.09$, $\left.\mathrm{CH}_{2} \mathrm{Cl}_{2}\right) .{ }^{1} \mathrm{H}$ NMR (500 MHz, $\left.\mathrm{CDCl}_{3}\right): \delta$ 7.29-7.26 (m, 2H), 7.21-7.18 (m, $3 \mathrm{H}), 4.68(\mathrm{~d}, J=9 \mathrm{~Hz}, 1 \mathrm{H}), 4.58(\mathrm{~d}, J=7.5 \mathrm{~Hz}, 1 \mathrm{H}), 4.30(\mathrm{~d}, J=2.5 \mathrm{~Hz}$,

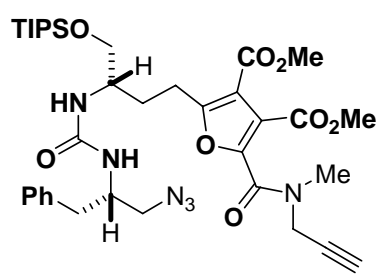
$2 \mathrm{H}), 4.11(\mathrm{~m}, 1 \mathrm{H}), 3.89(\mathrm{~s}, 3 \mathrm{H}), 3.84-3.80($ burried $\mathrm{m}, 1 \mathrm{H}), 3.80(\mathrm{~s}, 3 \mathrm{H}), 3.71(\mathrm{dd}, J=2.5,10 \mathrm{~Hz}$, $1 \mathrm{H}), 3.66(\mathrm{dd}, J=5,10 \mathrm{~Hz}, 1 \mathrm{H}), 3.42(\mathrm{dd}, J=2.5,12.5 \mathrm{~Hz}, 1 \mathrm{H}), 3.30(\mathrm{dd}, J=5,12.5 \mathrm{~Hz}, 1 \mathrm{H}), 3.19$ (br s, 3H), 3.14-3.07 (m, 1H), 3.03-2.97 (m, 1H), $2.85(\mathrm{dd}, J=6.5,13.5 \mathrm{~Hz}, 1 \mathrm{H}), 2.77(\mathrm{dd}, J=8$, $13.5 \mathrm{~Hz}, 1 \mathrm{H}), 2.27$ (br s, 1H), 2.01-1.94 (m, 1H), 1.90-1.83 (m, 1H), 1.10-1.02 (m, 21H). ${ }^{13} \mathrm{C}$ NMR (125 MHz, $\mathrm{CDCl}_{3}$ ): $\delta$ 163.5, 163.1, 162.3, 158.3, 156.7, 143.1, 137.3, 129.2, 128.657, 126.6, 123.8, $113.3,77.8,72.8,65.4,60.4,53.4,52.8,52.0,51.2,50.8,38.3,29.9,24.6,17.9,11.8$. IR (neat): 3370 , $3309,2945,2865,2100,1727,1637,1604,1552,1495,1449,1406,1285,1222,1112,1044$. MS (APCI+): Calc. for $\mathrm{C}_{36} \mathrm{H}_{52} \mathrm{~N}_{6} \mathrm{O}_{8} \mathrm{Si}(\mathrm{m} / \mathrm{z})$ 724.36. Found $(\mathrm{m} / \mathrm{z}) 725.45$.

Linear Precursor to 13a-c. The morpholino amide was prepared according to General Procedure D with the ester 4c $(0.31 \mathrm{~g}, 0.46$ $\mathrm{mmol})$. The crude organics were purified on silica eluting with 1:1 hexanes : EtOAc to give the amide as a colourless oil $(0.20 \mathrm{~g}, 62 \%) .{ }^{1} \mathbf{H}$ NMR (500 MHz, $\left.\mathbf{C D C l}_{3}\right): \delta$ 7.30-7.26 (m, 2H), 7.20-7.18 (m, 3H), 4.64

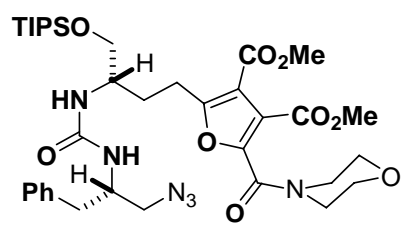
$(\mathrm{d}, J=9 \mathrm{~Hz}, 1 \mathrm{H}), 5.54(\mathrm{~d}, J=8.5 \mathrm{~Hz}, 1 \mathrm{H}), 4.11-4.10(\mathrm{~m}, 1 \mathrm{H}), 3.89(\mathrm{~s}, 3 \mathrm{H}), 3.81-3.80$ (burried $\mathrm{m}$, $1 \mathrm{H}), 3.81(\mathrm{~s}, 3 \mathrm{H}), 3.72(\mathrm{~d}, J=4.5 \mathrm{~Hz}, 8 \mathrm{H}), 3.68-3.67$ (burried m, $2 \mathrm{H}), 3.42(\mathrm{dd}, J=4.5,12.5 \mathrm{~Hz}$, $1 \mathrm{H}), 3.33(\mathrm{dd}, J=5,12.5 \mathrm{~Hz}, 1 \mathrm{H}), 3.13-3.07(\mathrm{~m}, 1 \mathrm{H}), 3.00-2.94(\mathrm{~m}, 1 \mathrm{H}), 2.86(\mathrm{dd}, J=7,13.5 \mathrm{~Hz}$, $1 \mathrm{H}), 2.77(\mathrm{dd}, J=8,13.5 \mathrm{~Hz}, 1 \mathrm{H}), 1.98-1.91(\mathrm{~m}, 1 \mathrm{H}), 1.90-1.82(\mathrm{~m}, 1 \mathrm{H}), 1.09-1.03(\mathrm{~m}, 21 \mathrm{H}) .{ }^{13} \mathrm{C}$ NMR (125 MHz, $\left.\mathrm{CDCl}_{3}\right): \delta 163.4,162.8,162.3,157.6,156.7,144.5,137.3,129.2,128.6,126.7$, 122.8, 113.2, 66.7, 65.4, 60.4, 53.4, 52.7, 52.1, 51.1, 50.9, 38.3, 30.0, 24.5, 17.9, 11.8. IR (neat): 3374, 2944, 2865, 2101, 1727, 1634, 1557, 1497, 1446, 1280, 1223, 1118, 1078, 1021. HRMS (ESI): Calc. for $\mathrm{C}_{36} \mathrm{H}_{54} \mathrm{~N}_{6} \mathrm{O} 9 \mathrm{Si}(\mathrm{m} / z)$ : 743.3800 . Found $(\mathrm{m} / \mathrm{z})$ : 743.3773 .

dimethyl 2-(((S)-1-azido-3-phenylpropan-2-yl)carbamoyl)-5-((S)-3-(3-methyl-3-(prop-2-ynyl) ureido)-4-(triisopropylsilyloxy)butyl)furan-3,4-dicarboxylate 14a. The azido-amide was prepared according to General Procedure D with the ester 5 $(223 \mathrm{mg}, 0.37 \mathrm{mmol})$ and (S)-azidomethylphenylalanine (117 $\mathrm{mg}, 0.55 \mathrm{mmol})$. The crude organics were purified on silica eluting with 1:1 hexanes : EtOAc to give the amide as a

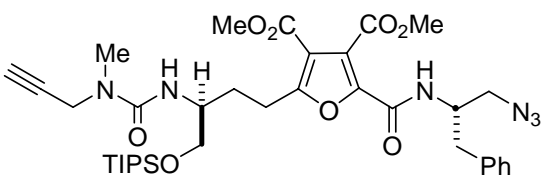
colourless oil (171 mg, 64\%). ${ }^{1} \mathbf{H}$ NMR (400 $\left.\mathbf{M H z}, \mathrm{CDCl}_{3}\right): \delta 8.33(\mathrm{~d}, J=8 \mathrm{~Hz}, 1 \mathrm{H}) ; 7.28-7.17(\mathrm{~m}$, $5 \mathrm{H}) ; 5.02(\mathrm{~d}, J=9.2 \mathrm{~Hz}, 1 \mathrm{H}) ; 4.49-4.42(\mathrm{~m}, 1 \mathrm{H}), 4.25(\mathrm{dd}, J=2.4,17.6 \mathrm{~Hz}, 1 \mathrm{H}) ; 4.10(\mathrm{dd}, J=2.4$, $17.6 \mathrm{~Hz}, 1 \mathrm{H}) ; 4.09-3.99(\mathrm{~m}, 1 \mathrm{H}) ; 3.94(\mathrm{~s}, 3 \mathrm{H}) ; 3.81(\mathrm{~s}, 3 \mathrm{H}) ; 3.76(\mathrm{dd}, J=3.6,10 \mathrm{~Hz}, 1 \mathrm{H}) ; 3.69$ (dd, $J$ $=2,10 \mathrm{~Hz}, 1 \mathrm{H}) ; 3.49(\mathrm{dd}, J=4.4,12.4 \mathrm{~Hz}, 1 \mathrm{H}) ; 3.42(\mathrm{dd}, J=5.2,12.4 \mathrm{~Hz}, 1 \mathrm{H}) ; 3.31(\mathrm{ddd}, J=4.4$, $7.2,14 \mathrm{~Hz}, 1 \mathrm{H}) ; 3.06(\mathrm{dd}, J=6.8,14 \mathrm{~Hz}, 1 \mathrm{H}) ; 3.00-2.87$ (burried $\mathrm{m}, 2 \mathrm{H}) ; 2.98(\mathrm{~s}, 3 \mathrm{H}) ; 2.25$ (t, $J=$ $2.4 \mathrm{~Hz}, 1 \mathrm{H}) ; 2.10-2.04(\mathrm{~m}, 1 \mathrm{H}) ; 1.84(\mathrm{ddd}, J=4.4,9.2,14 \mathrm{~Hz}, 1 \mathrm{H}) ; 1.10-1.00(\mathrm{~m}, 21 \mathrm{H}) .{ }^{13} \mathrm{C}$ NMR (100 MHz, $\left.\mathbf{C D C l}_{3}\right): \delta 164.6,163.0,162.6,157.4,156.9,143.2,137.8,129.5,128.7,126.8,122.2$, 113.9, 79.3, 72.4, 65.3, 53.3, 53.2, 52.2, 50.8, 50.6, 38.2, 37.7, 33.9, 29.7, 23.8, 18.2, 12.0. IR (Dep. 
$\mathrm{CDCl}_{3}$ ): 3275 (w); 2946, 2865, 2100, 1725, 1638, 1535 (all s). HRMS (ESI): Calc. for $\mathrm{C}_{36} \mathrm{H}_{53} \mathrm{~N}_{6} \mathrm{O}_{8} \mathrm{Si}$ $(\mathrm{m} / \mathrm{z}) 725.3694$. Found $(\mathrm{m} / \mathrm{z}) 725.3680$.

dimethyl 2-(((R)-1-azido-3-phenylpropan-2-yl)carbamoyl)-5((S)-3-(3-methyl-3-(prop-2-ynyl) (triisopropylsilyloxy)butyl)furan-3,4-dicarboxylate 14b.

The azido-amide was prepared according to General

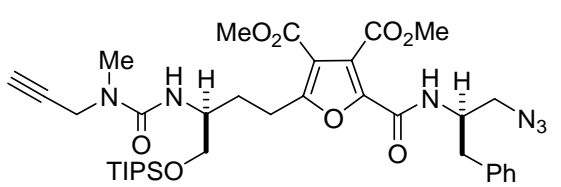
Procedure D with the ester 5 (154 mg, $0.27 \mathrm{mmol}$ ) and $(R)$-azidomethylphenylalanine $(74 \mathrm{mg}$, $0.55 \mathrm{mmol}$ ). The crude organics were purified on silica eluting with 1:1 hexanes : EtOAc to give the amide as a colourless oil $(119 \mathrm{mg}, 62 \%) .{ }^{1} \mathbf{H N M R}\left(\mathbf{5 0 0} \mathbf{~ M H z}, \mathbf{C D C l}_{3}\right): \delta 8.36 .(\mathrm{d}, J=8.0$ $\mathrm{Hz}, 1 \mathrm{H}) ; 7.29-7.18(\mathrm{~m}, 5 \mathrm{H}) ; 5.05(\mathrm{~d}, J=9.5 \mathrm{~Hz}, 1 \mathrm{H}) ; 4.46-4.40(\mathrm{~m}, 1 \mathrm{H}) ; 4.13(\mathrm{dd}, J=2.5,17.5 \mathrm{~Hz}$, $1 \mathrm{H}) ; 4.07$ (burried m, $1 \mathrm{H}) ; 4.06(\mathrm{dd}, J=2.5,17.5 \mathrm{~Hz}, 1 \mathrm{H}) ; 3.95(\mathrm{~s}, 3 \mathrm{H}) ; 3.81(\mathrm{~s}, 3 \mathrm{H}) ; 3.73$ (dd, $J=3.5$, $9.5 \mathrm{~Hz}, 1 \mathrm{H}) ; 3.66(\mathrm{dd}, J=2.5,9.5 \mathrm{~Hz}, 1 \mathrm{H}) ; 3.46(\mathrm{dd}, J=6,13 \mathrm{~Hz}, 1 \mathrm{H}) ; 3.39$ (dd, $J=4.5,13 \mathrm{~Hz}, 1 \mathrm{H})$; 3.30 (ddd, $J=5,7,13.8 \mathrm{~Hz}, 1 \mathrm{H}) ; 3.05$ (dd, $J=5,14 \mathrm{~Hz}, 1 \mathrm{H}) ; 2.97$ (dd, $J=8.5,14 \mathrm{~Hz}, 1 \mathrm{H}$ ); 2.96 (s, $3 \mathrm{H}) ; 2.93-2.88$ (buried m, $1 \mathrm{H}) ; 2.24(\mathrm{t}, J=2.5 \mathrm{~Hz}, 1 \mathrm{H}) ; 2.12-2.06(\mathrm{~m}, 1 \mathrm{H}) ; 1.88-1.81(\mathrm{~m}, 1 \mathrm{H}) ; 1.14-$ $1.01(\mathrm{~m}, 21 \mathrm{H}){ }^{13}{ }^{\mathbf{3}} \mathbf{~ N M R}\left(\mathbf{1 2 5} \mathbf{~ M H z}, \mathbf{C D C l}_{3}\right): \delta 164.5,162.9,162.5,157.4,156.9,143.2,137.6,129.4$, $128.7,126.8,122.2,113.9,79.23,72.4,65.2,53.3,52.9,52.2,50.9,50.6,38.2,34.0,29.5,23.8,18.2$, 11.9.IR (Dep. CDCl C): $_{3} 272$ (m), 2945, 2865, 2101, 1726, 1639, 1537, 1223 (all s). HRMS (ESI): Calc. for $\mathrm{C}_{36} \mathrm{H}_{53} \mathrm{~N}_{6} \mathrm{O}_{8} \mathrm{Si}(\mathrm{m} / z)$ 725.3694. Found $(\mathrm{m} / \mathrm{z}) 725.3727$.

Linear Precursor to 16. The azido-amide was prepared according to General Procedure D with the ester $5(107 \mathrm{mg}, 0.19$ $\mathrm{mmol}$ ) and azidomethyl-L-proline $(61 \mathrm{mg}, 0.38 \mathrm{mmol})$. The crude organics were purified on silica eluting with 2:1 hexanes : EtOAc to give the amide as a colourless oil (102 mg, $78 \%) .{ }^{1} \mathbf{H}$

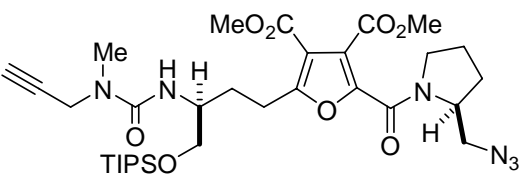
NMR (500 MHz, $\mathbf{C D C l}_{3}$ ): $\delta 4.94$ (d, $\left.J=8 \mathrm{~Hz}, 1 \mathrm{H}\right) ; 4.36$ (br s, 1H); 4.12 (dd, $\left.J=2,17.5 \mathrm{~Hz}, 1 \mathrm{H}\right) ; 4.06$ (dd, $J=2,17.5 \mathrm{~Hz}, 1 \mathrm{H}) ; 3.92(\mathrm{~s}, 3 \mathrm{H}) ; 3.92-3.83$ (burried m, 1H); 3.82 (burried m, 1H); $3.77(\mathrm{~s}, 3 \mathrm{H}$ ); $3.78-3.72(\mathrm{~m}, 2 \mathrm{H}) ; 3.64(\mathrm{dd}, J=6.5,12 \mathrm{~Hz}, 1 \mathrm{H}) ; 3.53(\mathrm{dd}, J=2.5,11.5 \mathrm{~Hz}, 1 \mathrm{H}) ; 3.21-3.14(\mathrm{~m}, 3 \mathrm{H})$; 3.02-2.96 (m, 1H); 2.91 (br s, 3H); $2.20(\mathrm{t}, J=2 \mathrm{~Hz}, 1 \mathrm{H}) ; 2.06-1.87(\mathrm{~m}, 7 \mathrm{H}) ; 1.12-1.02(\mathrm{~m}, 21 \mathrm{H}) .{ }^{13} \mathrm{C}$ NMR (125 MHz, $\left.\mathbf{C D C l}_{3}\right): \delta$ 164.4, 163.3, 162.3, 157.4, 157.1, 143.7, 125.1, 113.5, 79.4, 72.1, 72.0, 65.1, 57.8, 53.1, 52.1, 51.8, 51.6, 48.6, 37.9, 33.7, 30.2, 27.4, 25.0, 18.1, 12.0. IR (Dep. $\mathbf{C D C l}_{3}$ ): 3307 (br), 2945, 2865, 2103, 1732, 1634, 1519, 1455 (all s). HRMS (ESI): Calc. for $\mathrm{C}_{32} \mathrm{H}_{51} \mathrm{~N}_{6} \mathrm{O}_{8} \mathrm{Si}(\mathrm{m} / \mathrm{z}$ ) 675.3537. Found $(m / z) 675.3520$.

Linear azido-alkyne prescursor $17 \mathrm{a}(5 S, 9 S, 14 S)$ and $17 \mathrm{~b}(5 R, 9 R, 14 R)$. The amide $17 \mathrm{~b}$ was prepared according to general procedure $\mathrm{D}$; on $\mathbf{6 b}(131 \mathrm{mg}, 0.19$ $\mathrm{mmol})$ using (R)-azido-Phe $(60 \mathrm{mg}, 0.28 \mathrm{mmol})$ and the product purified on silica eluting with 1:1 Hexanes : EtOAc to give the amide as a white foam $(124 \mathrm{mg}, 81 \%) .(17 \mathrm{a} ; 5 S, 9 S, 14 S)\left[\alpha{ }^{25} \mathrm{D}=-6.74(\mathrm{c}=\right.$ $\left.0.46, \mathrm{CH}_{2} \mathrm{Cl}_{2}\right) ;(17 \mathrm{~b} ; 5 R, 9 R, 14 R) .[\alpha]^{25} \mathrm{D}=+47.7\left(\mathrm{c}=0.53, \mathrm{CH}_{2} \mathrm{Cl}_{2}\right) ; .{ }^{1} \mathbf{H}$ NMR (400 MHz, $\left.\mathrm{CDCl}_{3}\right): \delta 8.22(\mathrm{~d}, J=8 \mathrm{~Hz}, 1 \mathrm{H}) ; 7.32-7.18(\mathrm{~m}, 10 \mathrm{H})$;

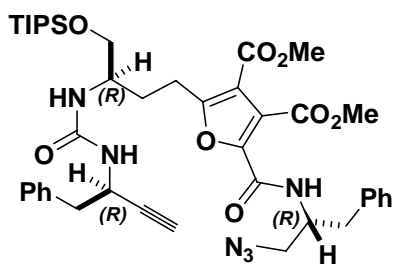
4.96-4.92 (m ,1H); $4.70(\mathrm{~d}, J=9.6 \mathrm{~Hz}, 1 \mathrm{H}) ; 4.61(\mathrm{~d}, J=8 \mathrm{~Hz}, 1 \mathrm{H}) ; 4.48-4.39(\mathrm{~m}, 1 \mathrm{H}) .4 .01-3.95$ (burried m, 1H); $3.94(\mathrm{~s}, 3 \mathrm{H}) ; 3.81(\mathrm{~s}, 3 \mathrm{H}) ; 3.73(\mathrm{dd}, J=3.6,10 \mathrm{~Hz}, 1 \mathrm{H}) ; 3.64(\mathrm{dd}, J=2.8,10 \mathrm{~Hz}$, $1 \mathrm{H}) ; 3.48(\mathrm{dd}, J=4.4,12.4 \mathrm{~Hz}, 1 \mathrm{H}) ; 3.41(\mathrm{dd}, J=5.2,12.4 \mathrm{~Hz}, 1 \mathrm{H}) ; 3.28(\mathrm{dt}, J=5.6,15.2 \mathrm{~Hz}, 1 \mathrm{H})$; 3.06 (burried dd, $J=6.8,8 \mathrm{~Hz}, 1 \mathrm{H}) ; 3.02(\mathrm{ddd}, J=6.4,10,15 \mathrm{~Hz}, 1 \mathrm{H}) ; 2.96$ (burried dd, $J=4,8$ $\mathrm{Hz}, 1 \mathrm{H}) ; 2.87$ (ddd, $J=5.2,10,15 \mathrm{~Hz}, 1 \mathrm{H}) ; 2.30$ (d, J = $2 \mathrm{~Hz}, 1 \mathrm{H}) ; 2.00-1.92(\mathrm{~m}, 1 \mathrm{H}) ; 1.85-1.78$ (m, 
1H); 1.09-1.03 (m, 21H). ${ }^{13}$ C NMR (100 MHz, $\left.\mathbf{C D C l}_{3}\right): \delta 164.6,163.1,162.6,156.9,156.5,143.4$, $137.7,136.5,130.0,129.5,128.8,128.6,127.2,126.9,122.1,114.1,83.3,72.7,66.1,53.3,53.2,52.3$, 50.9, 50.3, 43.5, 41.9, 37.8, 30.0, 23.8, 18.2, 12.1. IR (neat): 3270 (w); 2943, 2864 (both m); 2100, 1725, 1644, 1540 (all s). HRMS (ESI+): Calc. for $\mathrm{C}_{42} \mathrm{H}_{57} \mathrm{~N}_{6} \mathrm{O}_{8} \mathrm{Si}(\mathrm{m} / \mathrm{z})$ 801.4007; found (m/z) 801.4018.

Linear azido-alkyne prescursor $17 \mathrm{c}(5 S, 9 S, 14 R)$ and $17 \mathrm{~d}(5 R, 9 R, 14 S)$. The amide $\mathbf{1 7 d}$ was prepared according to general procedure $\mathrm{D}$; on ester 6c (131 mg, $0.19 \mathrm{mmol})$ using (S)-azido-Phe (60 mg, $0.28 \mathrm{mmol}$ ) and the product purified on silica eluting with 1:1 Hexanes : EtOAc to give the amide as a white foam $(118 \mathrm{mg}, 78 \%)$. (17c $5 S, 9 S, 14 R)[\alpha]^{25} \mathrm{D}=$ -2.81 (c = 0.64, $\left.\mathrm{CH}_{2} \mathrm{Cl}_{2}\right) ;(17 \mathrm{~d} 5 \mathrm{R}, 9 \mathrm{R}, 14 S)[\alpha]^{25} \mathrm{D}=+32.4^{\circ} \quad(\mathrm{c}=0.57$,

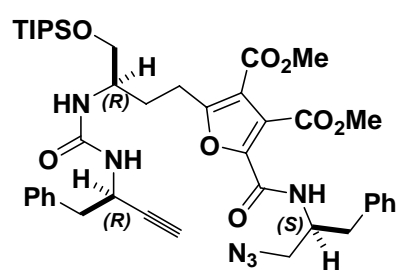
$\left.\mathrm{CH}_{2} \mathrm{Cl}_{2}\right) .{ }^{1} \mathrm{H}$ NMR (400 MHz, $\left.\mathrm{CDCl}_{3}\right): \delta 8.28(\mathrm{~d}, J=8.4 \mathrm{~Hz}, 1 \mathrm{H}) ; 7.32-7.19(\mathrm{~m}, 10 \mathrm{H}) ; 4.83$ (br dd, $J=6,12 \mathrm{~Hz}, 1 \mathrm{H}) ; 4.75(\mathrm{~d}, J=9.2 \mathrm{~Hz}, 1 \mathrm{H}) ; 4.62(\mathrm{~d}, J=8.4 \mathrm{~Hz}, 1 \mathrm{H}) ; 4.48-4.40(\mathrm{~m}, 1 \mathrm{H}) ; 4.09-4.00$ $(\mathrm{m}, 1 \mathrm{H}) ; 3.95(\mathrm{~s}, 3 \mathrm{H}) ; 3.80(\mathrm{~s}, 3 \mathrm{H}) ; 3.71(\mathrm{dd}, J=3.6,10 \mathrm{~Hz}, 1 \mathrm{H}) ; 3.62(\mathrm{dd}, J=2.8,10 \mathrm{~Hz}, 1 \mathrm{H}) ; 3.42$ $(\mathrm{d}, J=4.8 \mathrm{~Hz}, 2 \mathrm{H}) ; 3.29(\mathrm{dt}, J=5.6,15.2 \mathrm{~Hz}, 1 \mathrm{H}) ; 3.05(\mathrm{dd}, J 8,13.2 \mathrm{~Hz}, 1 \mathrm{H}) ; 3.00-2.94(\mathrm{~m}, 3 \mathrm{H})$; $2.86(\mathrm{ddd}, J=5.2,9.6 ; 15.2 \mathrm{~Hz}, 1 \mathrm{H}) ; 2.26(\mathrm{~d}, J=2 \mathrm{~Hz}, 1 \mathrm{H}) ; 2.02-1.93(\mathrm{~m}, 1 \mathrm{H}) ; 1.84-1.76(\mathrm{~m}, 1 \mathrm{H})$; 1.15-1.04 (m, 21H). ${ }^{13} \mathrm{C}$ NMR (100 MHz, $\left.\mathbf{C D C l}_{3}\right): \delta 164.6,163.1,162.6,156.9,156.5,143.4,137.5$, $136.5,130.0,129.5,128.8,127.2,126.9,122.0,114.0,83.4,72.6,65.9,53.3,52.9,52.3,50.9,50.3$, 43.6, 41.9, 38.2, 30.0, 23.7, 18.2, 12.0. IR (neat): 3270 (w); 2943, 2864 (both m); 2100, 1725, 1644, 1540 (all s). HRMS (ESI+): Calc. for $\mathrm{C}_{42} \mathrm{H}_{57} \mathrm{~N}_{6} \mathrm{O}_{8} \mathrm{Si}(\mathrm{m} / \mathrm{z})$ 801.4007; found (m/z) 801.4037.

Linear azido-alkyne prescursor $17 \mathrm{e}(5 S, 9 R, 14 S)$ and $17 f(5 R, 9 S, 14 R)$. The amide $\mathbf{1 7 f}$ was prepared according to general procedure $\mathrm{D}$; with 6d (131 mg, $0.19 \mathrm{mmol})$ using (R)-azido-Phe $(60 \mathrm{mg}, 0.28 \mathrm{mmol})$ and the product purified on silica eluting with 1:1 Hexanes:EtOAc to give the amide as a white foam $(112 \mathrm{mg}, 74 \%)$. (17e $5 S, 9 R, 14 S)[\alpha]^{25} \mathrm{D}=-$ 31.7 ( $\left.\mathrm{c}=0.42, \mathrm{CH}_{2} \mathrm{Cl}_{2}\right) ;(17 \mathrm{f}, 5 R, 9 S, 14 R)[\alpha]^{25} \mathrm{D}=+35.4 \quad(\mathrm{c}=0.48$,

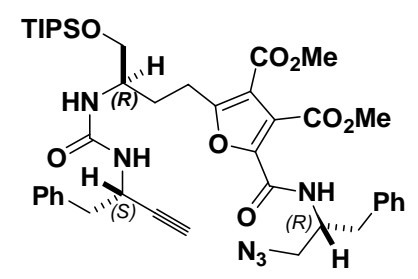
$\left.\mathrm{CH}_{2} \mathrm{Cl}_{2}\right) .{ }^{1} \mathrm{H}$ NMR (400 MHz, $\left.\mathrm{CDCl}_{3}\right): \delta 8.33(\mathrm{~d}, J=8 \mathrm{~Hz}, 1 \mathrm{H}) ; 7.28-7.18(\mathrm{~m}, 10 \mathrm{H}) ; 4.88(\mathrm{br} \mathrm{q}, J=$ $6 \mathrm{~Hz}, 1 \mathrm{H}) ; 4.77(\mathrm{~d}, J=9.2 \mathrm{~Hz}, 1 \mathrm{H}) ; 4.68(\mathrm{~d}, J=8.4 \mathrm{~Hz}, 1 \mathrm{H}) ; 4.67-4.40(\mathrm{~m}, 1 \mathrm{H}) ; 4.02-3.97(\mathrm{~m}, 1 \mathrm{H})$; $3.95(\mathrm{~s}, 3 \mathrm{H}) ; 3.81(\mathrm{~s}, 3 \mathrm{H}) ; 3.69(\mathrm{dd}, J=3.6,10 \mathrm{~Hz}, 1 \mathrm{H}) ; 3.64(\mathrm{dd}, J=2.8,10 \mathrm{~Hz}, 1 \mathrm{H}) ; 3.50(\mathrm{dd}, J=$ $4.4,12.4 \mathrm{~Hz}, 1 \mathrm{H}) ; 3.42(\mathrm{dd}, J=5.2,12.4 \mathrm{~Hz}, 1 \mathrm{H}) ; 3.27(\mathrm{ddd}, J=5.6,5.6,15.2 \mathrm{~Hz}, 1 \mathrm{H}) ; 3.06(\mathrm{dd}, J=$ $6.8,13.6 \mathrm{~Hz}, 1 \mathrm{H}) ; 3.03(\mathrm{~d}, J=6 \mathrm{~Hz}, 2 \mathrm{H}) ; 2.96(\mathrm{dd}, J=8.4,13.6 \mathrm{~Hz}, 1 \mathrm{H}) ; 2.83(\mathrm{ddd}, J=5.2,9.6$, $15.2 \mathrm{~Hz}, 1 \mathrm{H}) ; 2.29$ (d, J = $2 \mathrm{~Hz}, 1 \mathrm{H}) ; 1.98-1.91(\mathrm{~m}, 1 \mathrm{H}) ; 1.84(\mathrm{ddd}, J=4.8,9.2,14 \mathrm{~Hz}, 1 \mathrm{H}) ; 1.06-$ 1.01 (m, 21H). ${ }^{13} \mathrm{C}$ NMR (100 MHz, $\left.\mathbf{C D C l}_{3}\right): \delta 164.6,163.0,162.6,156.9,156.6,143.4,137.6$, $136.4,129.9,129.5,128.7,128.5,127.2,126.8,122.0,114.0,83.5,72.3,65.9,53.3,53.1,52.3,50.9$, 50.3, 43.5, 41.7, 37.7, 29.8, 23.7, 18.2, 12.0. IR (Dep. $\mathbf{C D C l}_{3}$ ): 3270, 2943, 2864 (all w), 2099, 1725, 1644, 1540, 1447, 1224 (all s). HRMS (ESI+): Calc. for $\mathrm{C}_{42} \mathrm{H}_{57} \mathrm{~N}_{6} \mathrm{O}_{8} \mathrm{Si}(\mathrm{m} / \mathrm{z})$ 801.4007; found ( $\mathrm{m} / \mathrm{z}$ ) 801.4025 .

Linear azido-alkyne prescursor $17 \mathrm{~g}(5 S, 9 R, 14 R)$ and $17 \mathrm{~h}(5 R, 9 S, 14 S)$. The amide $17 \mathrm{~h}$ was prepared according to general procedure D; using $(S)$-azido-Phe $(60 \mathrm{mg}, 0.28 \mathrm{mmol})$ and the product purified on silica eluting with 1:1 Hexanes:EtOAc to give the amide as a white foam $(108 \mathrm{mg}, 71 \%)$. (17h, $5 R, 9 S, 14 S)[\alpha]^{25} \mathrm{D}=+$ $12.7^{\circ}\left(\mathrm{c}=0.48, \mathrm{CH}_{2} \mathrm{Cl}_{2}\right) ;(17 \mathrm{~g}, 5 \mathrm{~S}, 9 \mathrm{R}, 14 \mathrm{R})[\alpha]^{25} \mathrm{D}=-11.4$ (c=0.42, $\left.\mathrm{CH}_{2} \mathrm{Cl}_{2}\right) .{ }^{1} \mathrm{H}$ NMR (400 MHz, $\left.\mathrm{CDCl}_{3}\right): \delta 8.31(\mathrm{~d}, J=8.4 \mathrm{~Hz}, 1 \mathrm{H}) ; 7.31-$

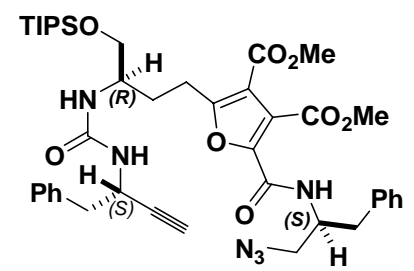


$7.16(\mathrm{~m}, 10 \mathrm{H}), 4.79$ (burried $\mathrm{m}, 1 \mathrm{H}) ; 4.75(\mathrm{~d}, J=8.8 \mathrm{~Hz}, 1 \mathrm{H}) ; 4.52(\mathrm{~d}, J=8 \mathrm{~Hz}, 1 \mathrm{H}) ; 4.47-4.04(\mathrm{~m}$, $1 \mathrm{H})$; 4.05-3.98 (m, 1H); $3.96(\mathrm{~s}, 3 \mathrm{H}) ; 3.82(\mathrm{~s}, 3 \mathrm{H}) ; 3.68(\mathrm{dd}, J=4,9.6 \mathrm{~Hz}, 1 \mathrm{H}) ; 3.63(\mathrm{dd}, J=2.8,9.6$ $\mathrm{Hz}, 1 \mathrm{H}) ; 3.45(\mathrm{dd}, J=5.6,12.4 \mathrm{~Hz}, 1 \mathrm{H}) ; 3.40(\mathrm{dd}, J=4.4,12.4 \mathrm{~Hz}, 1 \mathrm{H}) ; 3.27(\mathrm{ddd}, J=5.2,6.8,15.2$ $\mathrm{Hz}, 1 \mathrm{H}) ; 3.07(\mathrm{dd}, J=6.4,14 \mathrm{~Hz}, 1 \mathrm{H}), 2.98$ (app d, $J=6 \mathrm{~Hz}, 1 \mathrm{H}) ; 2.95$ (burried dd, $J=8.4,14 \mathrm{~Hz}$, $1 \mathrm{H}) ; 2.83(\mathrm{ddd}, J=5.2,9.6,15.2 \mathrm{~Hz}, 1 \mathrm{H}) ; 2.32(\mathrm{~d}, J=2 \mathrm{~Hz}, 1 \mathrm{H}) ; 2.02-1.93(\mathrm{~m}, 1 \mathrm{H}) ; 1.83(\mathrm{ddd}, J=$ 4.8, 9.6, $14 \mathrm{~Hz}, 1 \mathrm{H}) ; 1.07-1.01(\mathrm{~m}, 21 \mathrm{H}) .{ }^{13} \mathrm{C}$ NMR (100 $\left.\mathbf{M H z}, \mathbf{C D C l}_{3}\right): \delta 164.6,163.0,162.6$, $156.9,156.5,143.4,137.5,136.4,129.8,129.4,128.8,128.5,127.2,126.9,122.0,114.0,83.4,72.4$, 65.7, 53.3, 52.9, 52.3, 50.9, 50.3, 43.5, 41.8, 38.1, 29.7, 23.7, 18.2, 12.0. IR (neat): 3270 (w), 2943, 2864 (both m), 2100, 1725, 1644, 1540, 1445, 1224 (all s). HRMS (ESI+): Calc. for $\mathrm{C}_{42} \mathrm{H}_{57} \mathrm{~N}_{6} \mathrm{O}_{8} \mathrm{Si}$ $(\mathrm{m} / \mathrm{z})$ 801.4007; found $(\mathrm{m} / \mathrm{z}) 801.4013$.

Linear azido-alkyne prescursor 19a $(5 R, 9 R, 17 R)$. The amide 19a was prepared according to General Procedure D; on ester 7c (212 $\mathrm{mg}, 0.28 \mathrm{mmol}$ ) with (R)-azido-Phe (89 $\mathrm{mg}, 0.42 \mathrm{mmol})$. The product was puried on silica gel eluting with 2:1 hexanes: EtOAc to give the ester as a clear oil $(196 \mathrm{mg}, 82 \%)$. $[\alpha]^{25} \mathrm{D}=+47.2(\mathrm{c}=$ 1.12, $\left.\mathrm{CH}_{2} \mathrm{Cl}_{2}\right)$. ${ }^{1} \mathbf{H}$ NMR (400 $\left.\mathbf{M H z}, \mathbf{C D C l}_{3}\right): \delta 8.68(\mathrm{~d}, J=7.6 \mathrm{~Hz}$, $1 \mathrm{H}) ; 7.32-7.22(\mathrm{~m}, 10 \mathrm{H}) ; 7.08$ (br s, $1 \mathrm{H}) ; 6.19(\mathrm{~d}, J=8.0 \mathrm{~Hz}, 1 \mathrm{H})$;

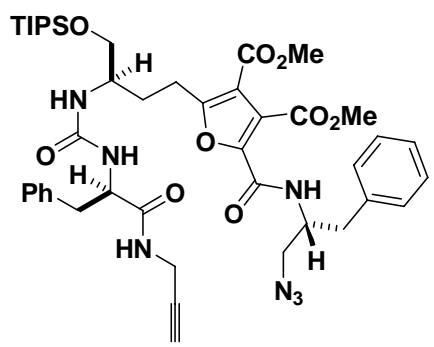
$5.51(\mathrm{~d}, J=8.8 \mathrm{~Hz}, 1 \mathrm{H}) ; 4.56(\mathrm{dd}, J=7.2,7.2 \mathrm{~Hz}, 1 \mathrm{H}) ; 4.49-4.40(\mathrm{~m}, 1 \mathrm{H}) ; 3.96(\mathrm{dd}, J=2.8,5.6 \mathrm{~Hz}$, $1 \mathrm{H}) ; 3.93(\mathrm{~s}, 3 \mathrm{H}) ; 3.91(\mathrm{dd}, J=3.2,4.8 \mathrm{~Hz}, 1 \mathrm{H}) ; 3.78(\mathrm{~s}, 1 \mathrm{H}) ; 3.75(\mathrm{dd}, J=2.8,4.8 \mathrm{~Hz}, 1 \mathrm{H}) ; 3.65$ $(\operatorname{app~d}, J=4 \mathrm{~Hz}, 2 \mathrm{H}) ; 3.61(\mathrm{dd}, J=3.6,9.2 \mathrm{~Hz}, 1 \mathrm{H}) ; 3.38(\mathrm{dd}, J=5.6,12.4 \mathrm{~Hz}, 1 \mathrm{H}) ; 3.08(\mathrm{dd}, J=6$, $13.6 \mathrm{~Hz}, 1 \mathrm{H}) ; 3.04(\mathrm{~d}, J=7.2 \mathrm{~Hz}, 1 \mathrm{H}) ; 2.86(\mathrm{dd}, J=7.2,14 \mathrm{~Hz}, 1 \mathrm{H}) ; 2.79$ (dt, J= 8.4, $14 \mathrm{~Hz}, 1 \mathrm{H})$; $2.12(\mathrm{t}, J=2.4 \mathrm{~Hz}, 1 \mathrm{H}) ; 1.96-1.88(\mathrm{~m}, 1 \mathrm{H}) ; 1.76-1.68(\mathrm{~m}, 1 \mathrm{H}) ; 1.12-1.03(\mathrm{~m}, 21 \mathrm{H}) .{ }^{13} \mathrm{C}$ NMR (100 $\left.\mathbf{M H z}, \mathrm{CDCl}_{3}\right): \delta 173.0,164.6,163.0,162.4,157.4,157.2,143.2,137.5,136.8,129.4,129.3,128.8$, 127.2, 126.9, 122.5, 114.0, 78.2, 72.1, 66.2, 55.9, 53.2, 53.1, 52.2, 50.9, 50.6, 39.5, 37.9, 30.2, 29.2, 24.4, 18.1, 12.0. IR (neat): 3328, 2945, 2865 (all m); 2102, 1731, 1647, 1540, 1456 (all s). HRMS (ESI+): Calc. for $\mathrm{C}_{44} \mathrm{H}_{60} \mathrm{~N}_{7} \mathrm{O}_{9} \mathrm{Si}(\mathrm{m} / \mathrm{z})$ 858.4222; found $(\mathrm{m} / \mathrm{z})$ 858.4208.

Linear azido-alkyne prescursor $19 \mathrm{~b}(5 R, 9 R, 17 S)$ : The amide $19 \mathrm{~b}$ was prepared according to General Procedure D; on ester 7c $(212 \mathrm{mg}$, $0.28 \mathrm{mmol})$ with $(S)$-azido-Phe $(89 \mathrm{mg}, 0.42 \mathrm{mmol})$. The product was puried on silica gel eluting with 2:1 hexanes: EtOAc to give the ester as a clear oil $(175 \mathrm{mg}, 73 \%) \cdot[\alpha]^{25} \mathrm{D}=+42.2\left(\mathrm{c}=1.40, \mathrm{CH}_{2} \mathrm{Cl}_{2}\right) \cdot{ }^{1} \mathbf{H}$ NMR (400 MHz, $\left.\mathrm{CDCl}_{3}\right): \delta 8.33(\mathrm{~d}, J=8.4 \mathrm{~Hz}, 1 \mathrm{H}) ; 7.29-7.16(\mathrm{~m}$, $10 \mathrm{H}) ; 6.29$ (br s); 5.52 (d, J = 8Hz, $1 \mathrm{H}) ; 5.14(\mathrm{~d}, J=8 \mathrm{~Hz}, 1 \mathrm{H}) ; 4.47-4.38$

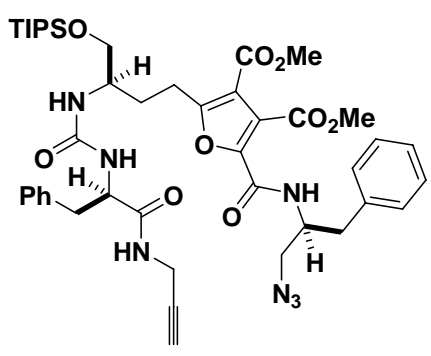
$(\mathrm{m}, 2 \mathrm{H}) ; 3.94(\mathrm{~s}, 3 \mathrm{H}) ; 3.91$ (burried $\mathrm{m}, 1 \mathrm{H}) ; 3.89(\mathrm{dd}, J=2.4,5.2 \mathrm{~Hz}, 1 \mathrm{H}) ; 3.83(\mathrm{dd}, J=2.8,5.2 \mathrm{~Hz}$, $1 \mathrm{H}) ; 3.78(\mathrm{~s}, 3 \mathrm{H}) ; 3.62(\mathrm{~m}, 2 \mathrm{H}) ; 3.42(\mathrm{~m}, 2 \mathrm{H}) ; 3.12$ (burried m, 1H); 3.08 (dd, J = 6.8, $14.4 \mathrm{~Hz}, 1 \mathrm{H})$; $3.02(\mathrm{~d}, J=7.2 \mathrm{~Hz}, 2 \mathrm{H}) ; 2.96(\mathrm{dd}, J=7.6,8.8 \mathrm{~Hz}, 1 \mathrm{H}) ; 2.81(\mathrm{dd}, J=8.8,16 \mathrm{~Hz}, 1 \mathrm{H}) ; 2.11(\mathrm{t}, J=2.8$ $\mathrm{Hz}, 1 \mathrm{H}) ; 1.87-1.78(\mathrm{~m}, 2 \mathrm{H}) ; 1.07-1.01(\mathrm{~m}, 21 \mathrm{H}) .{ }^{13} \mathbf{C} \mathbf{N M R}\left(\mathbf{1 0 0} \mathbf{~ M H z}, \mathbf{C D C l}_{3}\right): \delta 172.1,164.6$, $163.1,162.5,157.3,157.0,143.5,137.6,136.8,129.5,129.3,128.9,128.8,127.2,126.9,121.9,114.1$, 78.9, 71.9, 66.1, 55.6, 53.3, 53.1, 52.3, 50.8, 50.6, 39.1, 37.7, 30.2, 29.2, 24.0, 18.2, 12.0. IR (neat): 3274, 2943, 2865 (all w); 2100, 1728, 1647, 1540, 1224 (all s). HRMS (ESI+): Calc. for $\mathrm{C}_{44} \mathrm{H}_{60} \mathrm{~N}_{7} \mathrm{O}_{9} \mathrm{Si}$ $(\mathrm{m} / \mathrm{z})$ 858.4222; found $(\mathrm{m} / \mathrm{z}) 858.4184$.

Linear azido-alkyne prescursor 19c (5R, 9S, 17R): The amide 19c was prepared according to General Procedure D; on ester 7d (187 mg, $0.24 \mathrm{mmol})$ with $(R)$-azido-Phe $(78 \mathrm{mg}, 0.37 \mathrm{mmol})$. The product

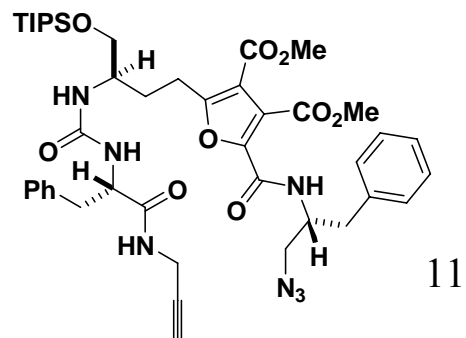


was puried on silica gel eluting with 2:1 hexanes: EtOAc to give the ester as a clear oil (164 mg, $78 \%) \cdot[\alpha]^{25} \mathrm{D}=+31.3\left(\mathrm{c}=1.58, \mathrm{CH}_{2} \mathrm{Cl}_{2}\right){ }^{\mathbf{1}} \mathbf{H}$ NMR (400 $\left.\mathbf{M H z}, \mathbf{C D C l}_{3}\right): \delta 8.21(\mathrm{~d}, J=8 \mathrm{~Hz}, 1 \mathrm{H})$; 7.31-7.09 (m, 10H); 6.48 (br s, 1H); 5.27 (br s, 1H); 5.00 (d, J = 7.6 Hz, 1H); 4.51 (dd, J = 7.2, 7.6 $\mathrm{Hz}, 1 \mathrm{H}) ; 4.48-4.39(\mathrm{~m}, 1 \mathrm{H}) ; 3.98(\mathrm{ddd}, J=2.4,2.4,5.6 \mathrm{~Hz}, 1 \mathrm{H}) ; 3.95(\mathrm{~s}, 3 \mathrm{H}) ; 3.81(\mathrm{~s}, 3 \mathrm{H}) ; 3.65-3.60$ $(\mathrm{m}, 2 \mathrm{H}) ; 3.45(\mathrm{dd}, J=4.4,12.8 \mathrm{~Hz}, 1 \mathrm{H}) ; 3.38(\mathrm{dd}, J=5.2 \mathrm{~Hz}, 12.8 \mathrm{~Hz}, 1 \mathrm{H}) ; 3.19(\mathrm{ddd}, J=5.6,5.6$, $15.2 \mathrm{~Hz}, 1 \mathrm{H}) ; 3.13(\mathrm{dd}, J=6.4,14 \mathrm{~Hz}, 1 \mathrm{H}) ; 3.04(\mathrm{dd}, J=6.8,14 \mathrm{~Hz}, 1 \mathrm{H}) ; 2.99-2.93(\mathrm{~m}, 3 \mathrm{H}) ; 2.77$ $(\mathrm{ddd}, J=7.2,12.4,14 \mathrm{~Hz}, 1 \mathrm{H}) ; 2.18(\mathrm{t}, J=2.8 \mathrm{~Hz}, 1 \mathrm{H}) ; 1.89-1.83(\mathrm{~m}, 2 \mathrm{H}) ; 1.08-1.01(\mathrm{~m}, 21 \mathrm{H}) .{ }^{13} \mathrm{C}$ NMR (100 MHz, $\left.\mathrm{CDCl}_{3}\right): \delta 171.9,164.6,163.0,162.6,157.4,156.9,143.6,137.4,136.8,129.4$, 129.3, 128.9, 128.7, 127.1, 127.0, 121.79, 114.1, 79.2, 71.9, 65.9, 55.4, 53.4, 53.0, 52.3, 50.8, 50.6, 38.5, 38.0, 29.9, 29.3, 23.6. 18.1, 12.0. IR (Dep. $\mathbf{C D C l}_{3}$ ): 3306, 2943, 2865 (all w), 2101, 1727, 1640, 1540, 1446, 1224 (all s). HRMS (ESI+): Calc. for $\mathrm{C}_{44} \mathrm{H}_{60} \mathrm{~N}_{7} \mathrm{O}_{9} \mathrm{Si}(\mathrm{m} / \mathrm{z})$ 858.4222; found (m/z) 858.4185.

Linear azido-alkyne prescursor 19d (5R, 9S, $17 S)$ : The amide 19d was prepared according to General Procedure D; on ester $7 \mathbf{d}(187 \mathrm{mg}, 0.24$ $\mathrm{mmol})$ with $(R)$-azido-Phe $(78 \mathrm{mg}, 0.37 \mathrm{mmol})$. The product was puried on silica gel eluting with 2:1 hexanes: EtOAc to give the ester as a clear oil $(143 \mathrm{mg}, 69 \%) .[\alpha]^{25} \mathrm{D}=+16.7$ (c = 0.92, $\left.\mathrm{CH}_{2} \mathrm{Cl}_{2}\right) .{ }^{1} \mathbf{H}$ NMR (400 MHz, $\left.\mathrm{CDCl}_{3}\right): \delta 8.21(\mathrm{~d}, J=8 \mathrm{~Hz}, 1 \mathrm{H}) ; 7.31-7.09(\mathrm{~m}, 10 \mathrm{H})$; 6.48 (br s, 1H); 5.27 (br s, 1H); 5.00 (d, $J=7.6 \mathrm{~Hz}, 1 \mathrm{H}) ; 4.51$ (dd, $J=7.2$,

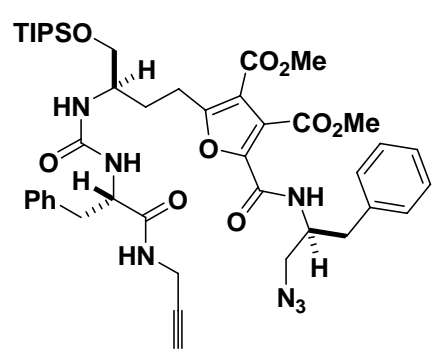
7.2 Hz, 1H); 4.48-4.39 (m, 1H); $3.98(\mathrm{ddd}, J=2.4,2.4,5.6 \mathrm{~Hz}, 1 \mathrm{H}) ; 3.95(\mathrm{~s}, 3 \mathrm{H}) ; 3.81(\mathrm{~s}, 3 \mathrm{H}) ; 3.65-$ $3.60(\mathrm{~m}, 2 \mathrm{H}) ; 3.45(\mathrm{dd}, J=4.4,12.8 \mathrm{~Hz}, 1 \mathrm{H}) ; 3.38(\mathrm{dd}, J=5.2,12.8 \mathrm{~Hz}, 1 \mathrm{H}) ; 3.19(\mathrm{ddd}, J=5.6,5.6$, $15.2 \mathrm{~Hz}, 1 \mathrm{H}) ; 3.13(\mathrm{dd}, J=6.4,14 \mathrm{~Hz}, 1 \mathrm{H}) ; 3.04(\mathrm{dd}, J=6.8,14 \mathrm{~Hz}, 1 \mathrm{H}) ; 2.99-2.93(\mathrm{~m}, 3 \mathrm{H}) ; 2.77$ $(\mathrm{ddd}, J=7.2,12.4,14 \mathrm{~Hz}, 1 \mathrm{H}) ; 2.18(\mathrm{t}, J=2.8 \mathrm{~Hz}, 1 \mathrm{H}) ; 1.89-1.83(\mathrm{~m}, 2 \mathrm{H}) ; 1.08-1.01(\mathrm{~m}, 21 \mathrm{H}) .{ }^{13} \mathrm{C}$ NMR (100 MHz, $\left.\mathrm{CDCl}_{3}\right): \delta 171.9,164.6,163.0,162.6,157.4,156.9,143.6,137.4,136.8,129.4$, $129.3,128.9,128.7,127.1,127.0,121.8,114.1,79.2,71.9,65.9,55.4,53.4,53.0,52.3,50.8,50.6,38.5$, 38.0, 29.9, 29.3, 23.6, 18.1, 12.0. IR (neat): 3306, 2943, 2865 (all m); 2101, 1727, 1640, 1540, 1224 (all s). HRMS (ESI+): Calc. for $\mathrm{C}_{44} \mathrm{H}_{60} \mathrm{~N}_{7} \mathrm{O}_{9} \mathrm{Si}(\mathrm{m} / \mathrm{z})$ 858.4222; found $(\mathrm{m} / \mathrm{z}) 858.4200$.

VI General procedure $E$ for the $\mathrm{Cu}(\mathrm{I})$ catalyzed macrocycloaddition reaction.

Macrocylcic triazole 10. To a solution of the alkyne $(100 \mathrm{mg}, 0.14$ $\mathrm{mmol})$ in PhMe (70 mL, to be $0.002 \mathrm{M})$ was added $i \operatorname{Pr}_{2} \mathrm{NEt}(4.91 \mathrm{~mL}$, $2.81 \mathrm{mmol})$ and $\mathrm{CuI}(13 \mathrm{mg}, 0.07 \mathrm{mmol})$. The mixture was stirred for $12 \mathrm{~h}$ at $\mathrm{rt}$, and then decanted from the insoluble $\mathrm{CuI}$ and concentrated. The mixture was purified on silica gel eluting with 2:1 hexanes : EtOAc $\rightarrow 100 \%$ EtOAc to give the triazole $(67 \mathrm{mg}, 67 \%)$ as an amorphous solid. Treatment of the product with HF.pyr and quenching with TMSOEt gave the free alcohol. ${ }^{1} \mathbf{H} \mathbf{~ N M R ~ ( 4 0 0 ~} \mathbf{~ M H z}$,

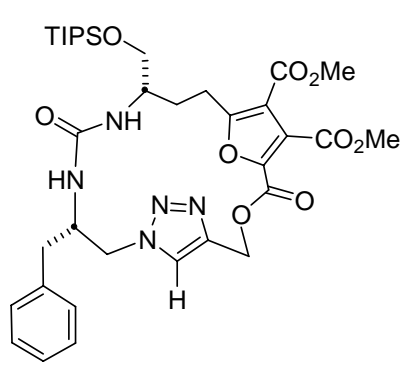
$\left.\mathrm{CDCl}_{3}\right): \delta 7.88(\mathrm{~s}, 1 \mathrm{H}) ; 7.31-7.23(\mathrm{~m}, 5 \mathrm{H}), 5.71(\mathrm{~d}, J=7.2 \mathrm{~Hz}, 1 \mathrm{H}), 5.41(\mathrm{~d}, J=10 \mathrm{~Hz}, 1 \mathrm{H}) ; 5.27$ $(1 / 2 \mathrm{ABq}, J=12 \mathrm{~Hz}, 1 \mathrm{H}) ; 5.20(1 / 2 \mathrm{ABq}, J=12 \mathrm{~Hz}, 1 \mathrm{H}) ; 4.90-4.79(\mathrm{~m}, 1 \mathrm{H}) ; 4.29(\mathrm{dd}, J=4,13.6$ $\mathrm{Hz}, 1 \mathrm{H}) ; 4.04(\mathrm{dd}, J=11.6,13.6 \mathrm{~Hz}, 1 \mathrm{H}) ; 3.93(\mathrm{~s}, 3 \mathrm{H}) ; 3.77(\mathrm{~s}, 3 \mathrm{H}) ; 3.65(\mathrm{dd}, J=4.4,10 \mathrm{~Hz}, 1 \mathrm{H})$; $3.60(\mathrm{dd}, J=2,10 \mathrm{~Hz}, 1 \mathrm{H}) ; 3.56-3.48$ (br m, 1H); 3.04 (app ddd, $J=2.4,11.2,16.2 \mathrm{~Hz}, 1 \mathrm{H})$; $2.97-$ 2.87 (m, 2H); 2.72 (app ddd, $J=2.4,6.4,16.2 \mathrm{~Hz}, 1 \mathrm{H}) ; 2.06-1.99$ (m, 1H); 1.64-1.57 (m, 1H), 1.051.00 (m, 21H). ${ }^{13} \mathrm{C}$ NMR (100 MHz, $\left.\mathbf{C D C l}_{3}\right): \delta 164.1,163.1,161.7,156.6,138.9,136.5,130.0$, $128.9,127.1,126.9,114.1,64.0,58.5,53.6,53.3,52.5,50.6,40.1,26.3,24.6,18.219,17.9,12.5,12.1$. 
IR (Dep. CDCl $)$ : 3358, 2943, 2865 (all m); 1729 (s); 1651, 1557, 1223 (all m). HRMS (ESI+) of free alcohol: Calc. for $\mathrm{C}_{26} \mathrm{H}_{30} \mathrm{~N}_{5} \mathrm{O}_{9}(\mathrm{~m} / \mathrm{z})$ 556.2043; found $(\mathrm{m} / \mathrm{z}) 556.2031$.

Macrocylcic triazole 12a. The triazole was prepared according to general procedure $\mathrm{E}$ with the azido-alkyne $(80 \mathrm{mg}, 0.11 \mathrm{mmol})$.The crude residue was purified on silica eluting with 9.5:0.5 EtOAc : iPrOH to yield the final macrocycle as a white solid $(60 \mathrm{mg}, 72 \%) .{ }^{1} \mathbf{H}$ NMR (500 MHz, $\left.\mathbf{C D C l}_{3}\right): 7.73(\mathrm{~s}, 1 \mathrm{H}), 7.30-7.26(\mathrm{~m}, 3 \mathrm{H})$, 7.24-7.21 (m, $2 \mathrm{H}), 4.90-4.86(\mathrm{~m}, 2 \mathrm{H}), 4.53(\mathrm{~d}, J=10 \mathrm{~Hz}, 1 \mathrm{H}), 4.36(\mathrm{~d}, J=14.5 \mathrm{~Hz}$, $1 \mathrm{H}), 3.91(\mathrm{~s}, 3 \mathrm{H}), 3.91-3.86$ (burried $\mathrm{m}, 2 \mathrm{H}), 3.81(\mathrm{~s}, 3 \mathrm{H}), 3.75-3.74(\mathrm{~m}$, $2 \mathrm{H}), 3.50(\mathrm{~m}, 1 \mathrm{H}), 3.14-3.13(\mathrm{~m}, 1 \mathrm{H}), 3.00-2.95$ (burried $\mathrm{m}, 2 \mathrm{H}), 2.95$

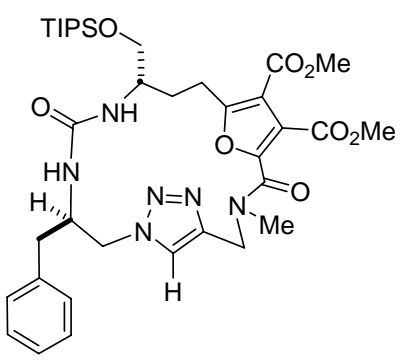
(s, 3H), $2.86(\mathrm{~m}, 1 \mathrm{H}), 2.07-2.05(\mathrm{~m}, 1 \mathrm{H}), 1.80(\mathrm{~m}, 2 \mathrm{H}), 1.05-1.01(\mathrm{~m}, 21 \mathrm{H}) .{ }^{13} \mathrm{C}$ NMR $(\mathbf{1 2 5} \mathbf{~ M H z}$, $\left.\mathrm{CDCl}_{3}\right): 163.8,162.9,161.9,158.2,157.0,143.9,143.4,137.6,129.2,128.7,126.8,125.1,124.5$, 113.8, 65.0, 53.0, 52.1, 51.8, 46.4, 39.4, 38.0, 33.8, 30.11, 29.6, 24.2, 17.9, 11.8. IR (neat): 3386, 2944, 2865, 1729, 1634, 1557, 1495, 1450, 1404, 1308, 1222, 1178, 1118, 1071, 1048. HRMS (ESI+): Calc. for $\mathrm{C}_{36} \mathrm{H}_{52} \mathrm{~N}_{6} \mathrm{O} 8 \mathrm{Si}(m / z)$ 725.3694. Found $(m / z) 725.3690$.

Macrocylcic triazole $12 \mathrm{~b}$. The triazole was prepared according to general procedure $\mathrm{E}$, with the azido-alkyne $(0.13 \mathrm{~g}, 0.18 \mathrm{mmol})$. The crude residue was purified on silica eluting with 9.5:0.5 EtOAc : $\mathrm{iPrOH}$ to yield the final macrocycle as a white solid (0.93 g, 72\%). ${ }^{1} \mathbf{H}$ NMR (500 MHz, $\left.\mathbf{C D C l}_{3}\right): \delta 7.75(\mathrm{~s}, 1 \mathrm{H}), 7.31-7.25(\mathrm{~m}, 5 \mathrm{H}), 5.16(\mathrm{~d}, J=7 \mathrm{~Hz}$, $1 \mathrm{H}), 5.00(\mathrm{~d}, J=6.5 \mathrm{~Hz}, 1 \mathrm{H}), 4.70-4.67(\mathrm{~m}, 1 \mathrm{H}), 4.60-4.51(\mathrm{~m}, 3 \mathrm{H}), 4.26$ $(\mathrm{dd}, J=8.5,13 \mathrm{~Hz}, 1 \mathrm{H}), 3.92(\mathrm{~s}, 3 \mathrm{H}), 3.79(\mathrm{~s}, 3 \mathrm{H}), 3.64$ (burried dd, $J=4$,

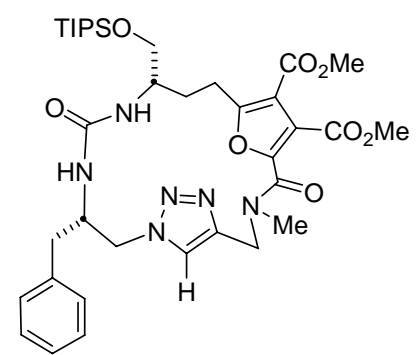
$13.5 \mathrm{~Hz}, 2 \mathrm{H}), 3.55$ (burried dd, $J=7,9.5 \mathrm{~Hz}, 1 \mathrm{H}), 3.01(\mathrm{~s}, 3 \mathrm{H}), 3.01-2.95$

(burried $\mathrm{m}, 3 \mathrm{H}), 2.42-2.41(\mathrm{~m}, 1 \mathrm{H}), 1.87(\mathrm{~m}, 1 \mathrm{H}), 1.73-1.67(\mathrm{~m}, 1 \mathrm{H}), 1.05-1.00(\mathrm{~m}, 21 \mathrm{H}) .{ }^{13} \mathrm{C}$ NMR (125 MHz, $\left.\mathrm{CDCl}_{3}\right): \delta 164.2,163.0,161.8,158.4,156.5,143.7,143.5,136.7,129.4,128.7$, 126.9, 125.0, 123.2, 113.7, 64.2, 53.4, 53.1, 52.0, 50.1, 46.7, 39.3, 34.2, 29.9, 29.7, 22.9, 17.9, 11.8. IR (neat): 3403, 2945, 2865, 1729, 1700, 1635, 1558, 1506, 1496, 1448, 1404, 1222, 1123, 1074, 1048. HRMS (ESI): Calc. for $\mathrm{C}_{36} \mathrm{H}_{52} \mathrm{~N}_{6} \mathrm{O}_{8} \mathrm{Si}(\mathrm{m} / z)$ 725.3694. Found $(\mathrm{m} / z) 725.3673$.

Macrocylcic triazole 15b (5S, 14S). Prepared according to General Procedure E, on the azido-alkyne 14a $(102 \mathrm{mg}, 0.14 \mathrm{mmol})$. The mixture was purified on silica eluting with 1:1 hexanes : EtOAc to give the triazole as a white foam $(65 \mathrm{mg}, 64 \%) .{ }^{1} \mathrm{HNMR}(400 \mathrm{MHz}$, $\left.\mathrm{CDCl}_{3}\right): \delta 7.59(\mathrm{~s}, 1 \mathrm{H}) ; 7.31-7.21(\mathrm{~m}, 5 \mathrm{H}) ; 7.04(\mathrm{~d}, \mathrm{~J}=8 \mathrm{~Hz}, 1 \mathrm{H}) ; 4.98$

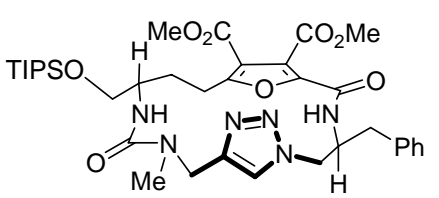
$(\mathrm{d}, J=15.6 \mathrm{~Hz}, 1 \mathrm{H}) ; 4.68(\mathrm{dd}, J=4 \mathrm{~Hz}, 13.6 \mathrm{~Hz}, 1 \mathrm{H}) ; 4.54(\mathrm{dd}, J=8.8,13.6 \mathrm{~Hz}, 1 \mathrm{H}) ; 4.47-4.38(\mathrm{~m}$, $1 \mathrm{H}) ; 4.28(\mathrm{~d}, J=8.8 \mathrm{~Hz}, 1 \mathrm{H}) ; 3.86(\mathrm{~s}, 3 \mathrm{H}) ; 3.80(\mathrm{~s}, 3 \mathrm{H}) ; 3.80-3.72$ (burried $\mathrm{m}, 1 \mathrm{H}) ; 3.66(\operatorname{app~d}, J=$ $4 \mathrm{~Hz}, 2 \mathrm{H}) ; 3.15(\mathrm{dd}, J=7.6,14 \mathrm{~Hz}, 1 \mathrm{H}) ; 3.10(\mathrm{ddd}, J=4.4,8,14 \mathrm{~Hz}, 1 \mathrm{H}) ; 3.0(\mathrm{dd}, J=6.8,14 \mathrm{~Hz}$, 1H); 2.98-2.90 (burried m, 1H); 2.69 (s, 3H); 2.14-1.97 (m, 2H); 1.15-0.90 (m, 21H). ${ }^{13} \mathrm{C}$ NMR (100 MHz, $\left.\mathrm{CDCl}_{3}\right): \delta 164.4,163.9,162.5,157.9,156.8,145.4,142.9,136.9,129.4,128.9,127.2,125.0$, 122.1, 113.9, 65.7, 53.3, 52.3, 52.2, 51.6, 44.7, 37.5, 34.7, 29.3, 25.7, 18.1, 12.0. IR (Dep. $\mathrm{CDCl}_{3}$ ): 2944, 1723, 1652, 1529 (all s). HRMS (ESI+): Calc. for $\mathrm{C}_{36} \mathrm{H}_{53} \mathrm{~N}_{6} \mathrm{O}_{8} \mathrm{Si}(\mathrm{m} / \mathrm{z})$ 725.3694. Found $(\mathrm{m} / \mathrm{z})$ 725.3700 . 


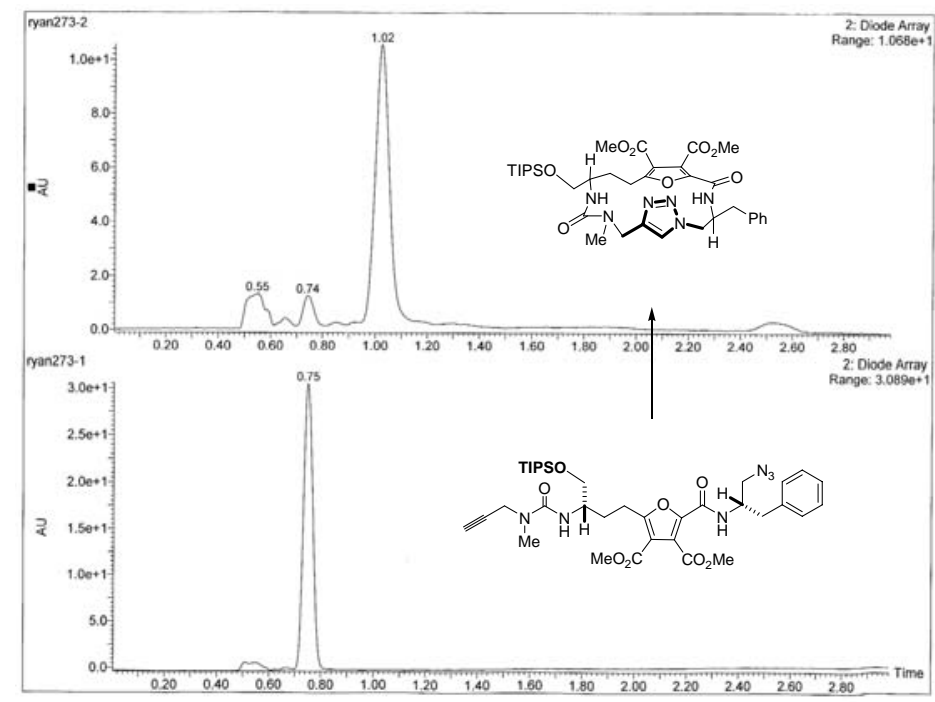

Representative crude SFC-trace for the macrocycloaddition of 15.

Macrocylcic triazole 15a $(5 S, 14 R)$. Prepared according to General Procedure E, on the azido-alkyne $14 \mathrm{~b}(78 \mathrm{mg}, 0.11 \mathrm{mmol})$. The mixture was purified on silica eluting with 1:1 hexanes : EtOAc to give the triazole as a white foam (53 mg, 68\%). ${ }^{1}$ HNMR (400 MHz, $\left.\mathbf{C D C l}_{3}\right): \delta 7.73(\mathrm{~s}, 1 \mathrm{H}) ; 7.40(\mathrm{~d}, J=7.2 \mathrm{~Hz}, 2 \mathrm{H}) ; 7.32(\mathrm{t}, J=7.6 \mathrm{~Hz}, 2 \mathrm{H})$;

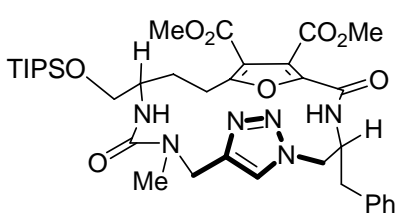
7.24 (t, $J=7.2 \mathrm{~Hz}, 1 \mathrm{H}) ; 6.29$ (br d, $J=7.6 \mathrm{~Hz}, 1 \mathrm{H}) ; 5.01$ (d, $J=15 \mathrm{~Hz}, 1 \mathrm{H}) ; 4.70-4.60$ (br m, 1H); 4.56$4.45(\mathrm{~m}, 2 \mathrm{H}) ; 3.93(\mathrm{~s}, 3 \mathrm{H}) ; 3.88(\mathrm{~d}, J=14.4 \mathrm{~Hz}, 1 \mathrm{H}) ; 3.82(\mathrm{~s}, 3 \mathrm{H}) ; 3.70(\mathrm{dd}, J=4.4,9.6 \mathrm{~Hz}, 1 \mathrm{H})$; $3.65-$ 3.60 (m, 2H), 3.17-3.11 (burried m, 1H); 3.08 (dd, J=9.6, $14 \mathrm{~Hz}, 1 \mathrm{H}$ ); 2.89 (s, 3H); 2.89-2.83 (burried $\mathrm{m}, 1 \mathrm{H}) ; 2.25-2.17(\mathrm{~m}, 1 \mathrm{H}) ; 1.96-1.87(\mathrm{~m}, 1 \mathrm{H}), 1.14-1.00(\mathrm{~m}, 21 \mathrm{H}) .{ }^{13} \mathbf{C}$ NMR (100 MHz, CDCl $\left.\mathbf{~}_{3}\right)$ $\delta 164.2,163.8,162.3,157.7,156.4,145.4,142.4,136.7,129.9,128.9,127.2,126.4,122.8,114.4,65.6$, 53.3, 52.3, 51.0, 50.5, 49.9, 44.8, 37.8, 35.3, 29.2, 25.2, 18.2, 12.0. IR (Dep. CDCl 3 ): 3364 (br, m), 2944, 2865, 1728, 1651, 1524 (all s). HRMS (ESI): Calc. for $\mathrm{C}_{36} \mathrm{H}_{53} \mathrm{~N}_{6} \mathrm{O}_{8} \mathrm{Si}(\mathrm{m} / z)$ 725.3694. Found $(\mathrm{m} / z) 725.3668$.

Macrocylcic triazole 16. The triazole was prepared according to General Procedure E, on the azido-alkyne $(70 \mathrm{mg}, 0.10 \mathrm{mmol})$. The mixture was purified on silica eluting with 1:1 hexanes : EtOAc to give the triazole as a white foam (58 mg, 83\%). ${ }^{1} \mathbf{H}$ NMR (400 MHz,

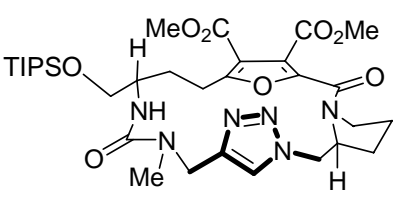
$\left.\mathbf{C D}_{3} \mathbf{C N}\right): \delta 7.37(\mathrm{~s}, 1 \mathrm{H}) ; 4.85(\mathrm{~d}, J=15.6 \mathrm{~Hz}, 1 \mathrm{H}) ; 4.76(\mathrm{~d}, J=4,14 \mathrm{~Hz}$, $1 \mathrm{H})$; 4.65-4.62 (m, 1H); 4.46-4.41 (m, 2H); $4.19(\mathrm{dd}, J=7.2,14 \mathrm{~Hz}, 1 \mathrm{H}) ; 3.77(\mathrm{~s}, 3 \mathrm{H}) ; 3.74(\mathrm{~s}, 3 \mathrm{H})$; 3.69 (dd, $J=4.4,6.6 \mathrm{~Hz}, 1 \mathrm{H}) ; 3.57-3.49$ (m, 4H); 3.33 (ddd, $J=3.6,8,11.2 \mathrm{~Hz}, 1 \mathrm{H}), 2.93($ br s, $1 \mathrm{H})$; 2.77 (ddd, $J=3.6,10,13.6 \mathrm{~Hz}, 1 \mathrm{H}) ; 2.53(\mathrm{~s}, 3 \mathrm{H}) ; 2.25-2.20$ (burried m, 1H); 2.04-1.96 (m, 2H); $1.83-$ $1.74(\mathrm{~m}, 1 \mathrm{H}) ; 1.67-1.60(\mathrm{~m}, 1 \mathrm{H}) ; 1.17-1.00(\mathrm{~m}, 21 \mathrm{H}) .{ }^{13} \mathbf{C}$ NMR (100 MHz, CDCl 3$): \delta 165.7,164.5$, $163.1,158.6,158.3,144.5,124.8,124.2,113.3,113.3,66.2,57.5,54.2,53.1,52.5,51.8,48.2,45.6$, 35.1, 29.5, 27.5, 26.9, 24.4, 18.3, 12.7. IR (neat): 3405 (w); 2945, 2865 (both m); 1724, 1637, 1449, 1220 (all s). HRMS (ESI+): Calc. for $\mathrm{C}_{32} \mathrm{H}_{51} \mathrm{~N}_{6} \mathrm{O}_{8} \mathrm{Si}(\mathrm{m} / \mathrm{z})$ 675.3537. Found (m/z) 675.3520.

Macrocylcic triazoles 18a $(5 S, 9 S, 14 S)$ and $18 b(5 R, 9 R, 14 R)$. The triazole 18a was prepared according to general procedure E from 17a (82 $\mathrm{mg}, 102 \mu \mathrm{mol})$ and purified on silica eluting with 1:1 hexanes : EtOAc $\rightarrow$ EtOAc to give the cyclo-dimer $(20 \mathrm{mg}, 25 \%)$ followed by the macrocycle as a white solid ( $48 \mathrm{mg}, 58 \%)$. $\mathbf{1 8 b}$ was prepared according to general

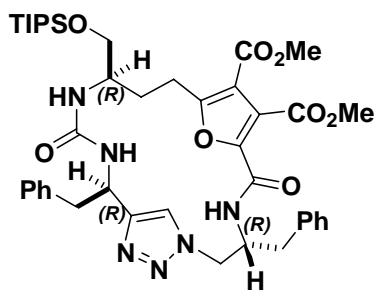


procedure E from $\mathbf{1 7 b}(70 \mathrm{mg}, 88 \mu \mathrm{mol})$ and purified on silica eluting with 1:1 Hexanes :EtOAc $\rightarrow$ EtOAc to give the cyclo-dimer $(20 \mathrm{mg}, 14 \%)$ followed by the macrocycle as a white solid (44 $\mathrm{mg}, 63 \%) .(\boldsymbol{R}, \boldsymbol{R}, \boldsymbol{R})[\alpha]^{25} \mathrm{D}=+38.8\left(\mathrm{c}=0.50, \mathrm{CH}_{2} \mathrm{Cl}_{2}\right) ;(S, S, S)[\alpha]^{25} \mathrm{D}=-30.2\left(\mathrm{c}=0.42, \mathrm{CH}_{2} \mathrm{Cl}_{2}\right)$. ${ }_{1}^{1} \mathrm{H}$ NMR (400 MHz, CD $\left.{ }_{3} \mathrm{OD}\right): \delta 7.59(\mathrm{~s}, 1 \mathrm{H}) ; 7.34-7.16(\mathrm{~m}, 10 \mathrm{H}) ; 6.36(\mathrm{~d}$ exch, $J=7.6 \mathrm{~Hz}, 1 \mathrm{H})$; $5.37(\mathrm{~d}$ exch, $J=8.8 \mathrm{~Hz}, 1 \mathrm{H}) ; 5.10(\mathrm{br} \mathrm{dd}, J=4.8,9.2 \mathrm{~Hz}, 1 \mathrm{H}) ; 4.66(\mathrm{dd}, J=4.4,13.6 \mathrm{~Hz}, 1 \mathrm{H})$; 4.58-4.52 (m, 1H); $4.45(\mathrm{dd}, J=7.2,13.6 \mathrm{~Hz}, 1 \mathrm{H}) ; 3.85(\mathrm{~s}, 3 \mathrm{H}) ; 3.79(\mathrm{~s}, 3 \mathrm{H}) ; 3.73-3.66$ (br m, $1 \mathrm{H})$; $3.62(\mathrm{dd}, J=4.4,10 \mathrm{~Hz}, 1 \mathrm{H}) ; 3.51(\mathrm{dd}, J=5.6,10 \mathrm{~Hz}, 1 \mathrm{H}) ; 3.37(\mathrm{dd}, J=4.8,14.4 \mathrm{~Hz}, 1 \mathrm{H}) ; 3.26$ $(\mathrm{dd}, J=4,8.8 \mathrm{~Hz}, 1 \mathrm{H}) ; 3.21(\mathrm{dd}, J=3.6,8.8 \mathrm{~Hz}, 1 \mathrm{H}) ; 3.03(\mathrm{dd}, J=7.2,14.4 \mathrm{~Hz}, 1 \mathrm{H}), 3.01-2.93(\mathrm{~m}$, $4 \mathrm{H}) ; 2.87(\mathrm{ddd}, J=3.6,8.4,14.4 \mathrm{~Hz}, 1 \mathrm{H}) ; 2.23-2.17(\mathrm{~m}, 1 \mathrm{H}) ; 1.98-1.89(\mathrm{~m}, 1 \mathrm{H}) ; 1.10-0.98(\mathrm{~m}$, 21H). ${ }^{13} \mathrm{C}$ NMR (100 MHz, $\left.\mathbf{C D C l}_{3}\right): \delta 164.7,163.9,162.4,157.1,156.8,149.8,142.1,137.7,136.9$, $129.5,129.4,128.9,128.6,127.2,126.8,123.9,114.5,65.9,53.4,52.6,52.3,51.4,51.0,47.8,40.3$, 37.4, 29.2, 25.0, 18.2, 12.0. IR (Dep. CDCl $_{3}$ ): 3389 (w), 2943, 2864 (both m), 1727, 1662, 1542, 1447, 1223, 1080 (all s).HRMS (ESI+): Calc. for $\mathrm{C}_{42} \mathrm{H}_{57} \mathrm{~N}_{6} \mathrm{O}_{8} \mathrm{Si}(\mathrm{m} / \mathrm{z})$ 801.4007; found $(\mathrm{m} / \mathrm{z})$ 801.4021.

(5R, 9R, 14R -cyclo dimer): ${ }^{1} \mathrm{H}$ NMR (400 MHz, $\left.\mathrm{CD}_{3} \mathrm{OD}\right): \delta 8.84(\mathrm{~d}$ exch, $J=8.8 \mathrm{~Hz}, 2 \mathrm{H}) ; 7.39$ (s, 2H); 7.32-7.09 (m, 20H); 6.23 (d exch, $J$ $=8.8 \mathrm{~Hz}, 2 \mathrm{H}) ; 5.87(\mathrm{~d}$ exch, $J=9.2 \mathrm{~Hz}, 2 \mathrm{H}) ; 5.26-5.23(\mathrm{~m}, 2 \mathrm{H}) ; 4.55$ $(\operatorname{app~d}, J=6.8 \mathrm{~Hz}, 4 \mathrm{H}) ; 4.36(\mathrm{dd}, J=10.4,14.4 \mathrm{~Hz}, 2 \mathrm{H}), 3.81(\mathrm{~s}, 6 \mathrm{H})$; $3.78(\mathrm{~s}, 6 \mathrm{H}) ; 3.66-3.62(\mathrm{~m}, 2 \mathrm{H}) ; 3.51(\mathrm{dd}, J=4.8,10 \mathrm{~Hz}, 2 \mathrm{H}) ; 3.44$ $(\mathrm{dd}, J=6.4,10 \mathrm{~Hz}, 2 \mathrm{H}) ; 3.27$ (burried d, $J=5.2 \mathrm{~Hz}, 2 \mathrm{H}) ; 3.07-2.89$ $(\mathrm{m}, 10 \mathrm{H}) ; 2.33-2.58(\mathrm{~m}, 2 \mathrm{H}) ; 1.83-1.77(\mathrm{~m}, 2 \mathrm{H}) ; 1.60-1.54(\mathrm{~m}, 2 \mathrm{H})$;

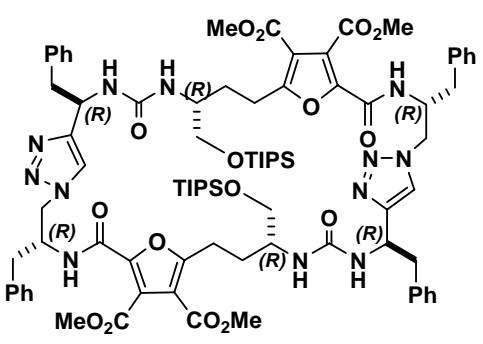
$1.01-1.03(\mathrm{~m}, 42 \mathrm{H})$.

Macrocylcic triazoles $18 \mathrm{c}(5 S, 9 S, 14 R)$ and $18 \mathrm{~d}(5 R, 9 R, 14 S)$. The triazole $18 \mathrm{c}$ was prepared according to general procedure E from $17 \mathrm{c}(60$ $\mathrm{mg}, 75 \mu \mathrm{mol})$ and purified on silica eluting with 9:1 EtOAc : hexanes gave the macrocycle as a white foam $(32 \mathrm{mg}, 53 \%)$. 18d was prepared according to general procedure $\mathrm{E}$ from $17 \mathrm{~d}(73 \mathrm{mg}, 91 \mu \mathrm{mol})$ and purified on silica eluting with 1:1 Hexanes :EtOAc $\rightarrow$ EtOAc to give the

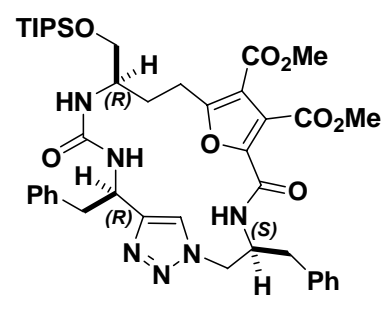
macrocycle as a white solid $39 \mathrm{mg}, 54 \%) .(\boldsymbol{R}, \boldsymbol{R}, \boldsymbol{S})[\alpha]^{25} \mathrm{D}=+6.1 \quad\left(\mathrm{c}=0.81, \mathrm{CH}_{2} \mathrm{Cl}_{2}\right) ;(S, S, R)$ $[\alpha]^{25} \mathrm{D}=-3.2\left(\mathrm{c}=0.42, \mathrm{CH}_{2} \mathrm{Cl}_{2}\right) .{ }^{1} \mathrm{HNMR}\left(400 \mathrm{MHz}, \mathrm{CD}_{3} \mathrm{OD}\right): \delta 7.63(\mathrm{br} \mathrm{s}, 1 \mathrm{H}) ; 7.34-7.16(\mathrm{~m}$, $10 \mathrm{H}) ; 6.30(\mathrm{~d}$ exch, $J=6 \mathrm{~Hz}) ; 5.65$ (d, exch., $J=8 \mathrm{~Hz}, 1 \mathrm{H}) ; 5.07(\mathrm{br} \mathrm{s}, 1 \mathrm{H}) ; 4.60(\mathrm{~d}, J=5.6 \mathrm{~Hz}, 2 \mathrm{H})$; 4.24 (quintet, $J=6.80 \mathrm{~Hz}, 1 \mathrm{H}) ; 3.89(\mathrm{~s}, 3 \mathrm{H}) ; 3.87-3.82$ (burried m, $1 \mathrm{H}) ; 3.79(\mathrm{~s}, 3 \mathrm{H}) ; 3.58(\mathrm{dd}, J=$ 3.6, $9.6 \mathrm{~Hz}, 1 \mathrm{H}) ; 3.45(\mathrm{dd}, J=5.6,9.6 \mathrm{~Hz}, 1 \mathrm{H}) ; 3.36-3.33(\mathrm{~m}, 1 \mathrm{H}) ; 3.23(\mathrm{br} \mathrm{d}, J=10.4 \mathrm{~Hz}, 1 \mathrm{H}) ; 3.13$ $(\mathrm{dd}, J=7.2,13.6 \mathrm{~Hz}, 1 \mathrm{H}) ; 3.05-2.96(\mathrm{~m}, 4 \mathrm{H}) ; 2.36-2.28(\mathrm{~m}, 1 \mathrm{H}) ; 1.85-1.78(\mathrm{~m}, 1 \mathrm{H}) ; 1.06-0.99(\mathrm{~m}$, 21H). ${ }^{13}$ C NMR (100 MHz, $\left.\mathbf{C D C l}_{3}\right)$ : $\delta 164.2,163.9,162.2,157.1,156.4,149.0,142.1,137.9,136.7$, $129.8,129.4,128.9,128.6,127.2,126.7,123.3,114.4,65.6,53.3,52.3,50.6,50.4,50.2,47.3,38.5$, 38.0, 28.8, 25.1, 18.1, 12.0. IR (Dep. CDCl$_{3}$ ): 3307 (w), 2943, 2864 (both m), 1728, 1653, 1533, 1447, 1221, 1118 (all s).HRMS (ESI+): Calc. for $\mathrm{C}_{42} \mathrm{H}_{57} \mathrm{~N}_{6} \mathrm{O} 8 \mathrm{Si}(\mathrm{m} / \mathrm{z})$ 801.4007; found $(\mathrm{m} / \mathrm{z})$ 801.3984.

Macrocylcic triazoles $18 \mathrm{e}(5 S, 9 R, 14 S)$ and $18 \mathrm{f}(5 R, 9 S, 14 R)$. The triazole 18e was prepared according to general procedure $\mathrm{E}$ from 17e $(52 \mathrm{mg}, 64 \mu \mathrm{mol})$ and purified on silica eluting with 9:1 EtOAc : hexanes gave the macrocycle as a white foam ( $35 \mathrm{mg}$, 68\%). 18f was prepared according to general procedure $\mathrm{E}$ from $17 \mathrm{f}(54 \mathrm{mg}, 67$ $\mu \mathrm{mol})$ and purified on silica eluting with 1:1 Hexanes : EtOAc $\rightarrow$

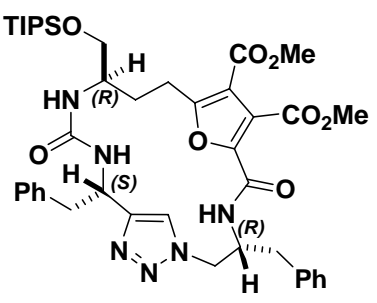
EtOAc to give the macrocycle as a white solid ( $34 \mathrm{mg}$, 63\%) preceded by the cyclodimer $(6 \mathrm{mg}$, 
$12 \%) .(R, S, S)[\alpha]^{25} \mathrm{D}=-34.2\left(\mathrm{c}=0.50, \mathrm{CH}_{2} \mathrm{Cl}_{2}\right) ;(S, R, R)\left[\alpha{ }^{25} \mathrm{D}=+48.7\left(\mathrm{c}=0.39, \mathrm{CH}_{2} \mathrm{Cl}_{2}\right) .{ }^{1} \mathbf{H}\right.$ NMR (500 MHz, DMSO- $\left.d_{6}, 353 \mathrm{~K}\right): \delta 8.13(\mathrm{~d}, J=7.5 \mathrm{~Hz}, 1 \mathrm{H}) ; 7.97(\mathrm{~s}, 1 \mathrm{H}) ; 730-7.13(\mathrm{~m}, 10 \mathrm{H})$, $6.14(\mathrm{~d}, J=7 \mathrm{~Hz}, 1 \mathrm{H}) ; 5.33(\mathrm{~d}, J=8.5 \mathrm{~Hz}, 1 \mathrm{H}) ; 4.62-4.60(\mathrm{br} \mathrm{s}, 1 \mathrm{H}) ; 4.57(\operatorname{app~d}, J=7 \mathrm{~Hz}, 2 \mathrm{H})$; 4.18-4.13 (m, 1H); 3.77-3.70 (burried $\mathrm{m}, 1 \mathrm{H}) ; 3.75(\mathrm{~s}, 3 \mathrm{H}) ; 3.72(\mathrm{~s}, 3 \mathrm{H}) ; 3.49-3.42(\mathrm{~m}, 1 \mathrm{H}) ; 3.39(\mathrm{t}, J$ $=8 \mathrm{~Hz}, 1 \mathrm{H}) ; 3.35(\mathrm{dd}, J=12.6,13 \mathrm{~Hz}, 1 \mathrm{H}) ; 3.08-2.98(\mathrm{~m}, 4 \mathrm{H}) ; 2.91(\mathrm{ddd}, J=4,7,11.5 \mathrm{~Hz}, 1 \mathrm{H})$; $2.21(\mathrm{ddd}, J=4,8.5,14 \mathrm{~Hz}, 1 \mathrm{H}) ; 1.78-1.71(\mathrm{~m}, 1 \mathrm{H}) ; 1.10-1.00(\mathrm{~m}, 21 \mathrm{H}) .{ }^{13} \mathrm{C}$ NMR (100 MHz, CD $_{3}$ CN): $\delta 164.8,164.7,162.9,157.4,143.2,140.1,138.9,131.4,130.3,130.1,129.7,129.4,129.1$, 128.2, 127.5, 127.2, 114.5, 68.7, 66.4, 53.3, 52.6, 52.2, 41.3, 39.6, 37.8, 31.1, 29.6, 29.4, 25.8, 24.5, 23.6, 18.4, 12.7. IR (Dep. CDCl $_{3}$ ): 3401 (w), 2943, 2864 (both m), 1725, 1653, 1541, 1445, 1220, 1072 (all s). HRMS (ESI+): Calc. for $\mathrm{C}_{42} \mathrm{H}_{57} \mathrm{~N}_{6} \mathrm{O}_{8} \mathrm{Si}(\mathrm{m} / \mathrm{z})$ 801.4007; found $(\mathrm{m} / \mathrm{z})$ 801.4042.

Macrocylcic triazoles $18 \mathrm{~g}(5 S, 9 R, 14 R)$ and $18 \mathrm{~h}(5 R, 9 S, 14 S)$. The triazole $18 \mathrm{~g}$ was prepared according to general procedure $\mathrm{E}$ from $\mathbf{1 7 g}(51 \mathrm{mg}, 64 \mu \mathrm{mol})$ and purified on silica eluting with 9:1 EtOAc : hexanes gave the macrocycle as a white foam ( $35 \mathrm{mg}, 68 \%) . \mathbf{1 8 d}$ was prepared according to general procedure E from $17 \mathrm{~d}(62 \mathrm{mg}, 77$ $\mu \mathrm{mol})$ and purified on silica eluting with 1:1 Hexanes : EtOAc $\rightarrow$

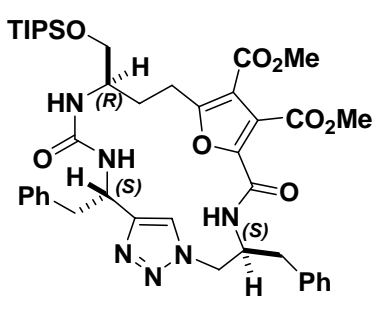
EtOAc to give the macrocycle as a white solid $(51 \mathrm{mg}, 83 \%) .(R, S, S)[\alpha]^{25} \mathrm{D}=-12.8(\mathrm{c}=0.50$, $\left.\mathrm{CH}_{2} \mathrm{Cl}_{2}\right) ;(S, R, R)[\alpha]^{25} \mathrm{D}=+15.7\left(\mathrm{c}=0.55, \mathrm{CH}_{2} \mathrm{Cl}_{2}\right) .{ }^{1} \mathrm{H}$ NMR $\left(400 \mathrm{MHz}, \mathrm{CD}_{3} \mathrm{CN}\right): \delta 7.83(\mathrm{~s}, 1 \mathrm{H}) ;$ 7.34-7.22 (m, 10H); 7.15 (br s, 1H); 5.00 (br s, 1H); 4.61-4.52 (m, 2H); 4.49 (dd, J = 5.6, $14.4 \mathrm{~Hz}$, $1 \mathrm{H}) ; 3.83(\mathrm{~s}, 3 \mathrm{H}) ; 3.78(\mathrm{~s}, 3 \mathrm{H}) ; 3.65(\mathrm{dd}, J=4,9.6 \mathrm{~Hz}, 1 \mathrm{H}) ; 3.42(\mathrm{dd}, J=6.8,9.6 \mathrm{~Hz}, 1 \mathrm{H}) ; 3.26(\mathrm{dd}$, $J=4.8,13.2 \mathrm{~Hz}, 1 \mathrm{H}) ; 3.23-3.10(\mathrm{~m}, 3 \mathrm{H}) ; 2.97(\mathrm{dd}, J=6.4,13.2 \mathrm{~Hz}, 1 \mathrm{H}) ; 2.88(\mathrm{dd}, J=3.6,11.2 \mathrm{~Hz}$, $1 \mathrm{H}) ; 2.82(\mathrm{dd}, J=4.8,8 \mathrm{~Hz}, 1 \mathrm{H}) ; 2.35-2.28(\mathrm{~m}, 1 \mathrm{H}) ; 2.15$ (br s, $1 \mathrm{H}) ; 1.87-1.74(\mathrm{~m}, 1 \mathrm{H}) ; 1.1-1.05(\mathrm{~m}$, 21H). ${ }^{13}$ C NMR (100 MHz, CD $_{3}$ CN): $\delta 164.7,164.6,162.9,157.7,157.1,149.0,142.1,138.9,131.9$, 130.4, 130.2, 129.4, 129.1, 127.5, 127.1, 124.0, 114.8, 70.1, 66.1, 53.3, 52.6, 52.3, 51.7, 51.4, 51.1, 41.3, 38.2, 29.5, 25.5, 18.4, 12.6. IR (neat): 3324 (w), 2943, 2864 (both m), 1727, 1651, 1533, 1445, 1221, 1072 (all s). HRMS (ESI+): Calc. for $\mathrm{C}_{42} \mathrm{H}_{57} \mathrm{~N}_{6} \mathrm{O}_{8} \mathrm{Si}(\mathrm{m} / \mathrm{z})$ 801.4007; found $(\mathrm{m} / \mathrm{z})$ 801.4016.

Macrocylcic triazole 20a $(5 R, 9 R, 17 R)$. The triazole 20a was prepared according to general procedure $\mathrm{E}$ from $19 \mathrm{a}(73 \mathrm{mg}, 85 \mu \mathrm{mol})$ and purified on silica eluting with 1:1 Hexanes : EtOAc $\rightarrow$ EtOAc to give the macrocycle as a white solid $(44 \mathrm{mg}, 60 \%)$. [In all cases the cyclodimer preceeded the monomer on silica]. $(\boldsymbol{R}, \boldsymbol{R}, \boldsymbol{R})[\alpha]^{25} \mathrm{D}=+70.5\left(\mathrm{c}=0.74, \mathrm{CH}_{2} \mathrm{Cl}_{2}\right)$. ${ }^{1}$ H NMR (500 MHz, DMSO-d $\left.d_{6}, 333 \mathrm{~K}\right): \delta 8.17(\mathrm{~d}, J=8 \mathrm{~Hz}, 1 \mathrm{H}) ; 8.02$ (br s); $7.74(\mathrm{~s}, 1 \mathrm{H}) ; 7.34-7.10(\mathrm{~m}, 10 \mathrm{H}) ; 6.24(\mathrm{~d}, J=8 \mathrm{~Hz}, 1 \mathrm{H}) ; 5.82(\mathrm{~d}, J=9 \mathrm{~Hz}$,

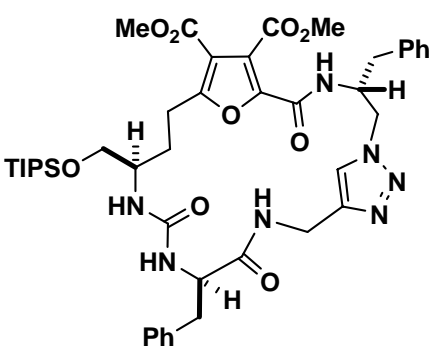
$1 \mathrm{H}) ; 4.58(\mathrm{dd}, J=8,15.5 \mathrm{~Hz}, 1 \mathrm{H}) ; 4.55-4.49(\mathrm{~m}, 2 \mathrm{H}) ; 4.68-4.43(\mathrm{~m}, 1 \mathrm{H}) ; 4.40(\mathrm{dd}, J=7,16.5 \mathrm{~Hz}$, $1 \mathrm{H}) ; 4.28(\mathrm{dd}, J=5.5 \mathrm{~Hz}, 15.5 \mathrm{~Hz}, 1 \mathrm{H}) ; 4.27-4.21(\mathrm{~m}, 1 \mathrm{H}) ; 3.75(\mathrm{~s}, 3 \mathrm{H}) ; 3.74(\mathrm{~s}, 3 \mathrm{H}) ; 3.73-3.69(\mathrm{~m}$, 2H); $3.67(\mathrm{dd}, J=3,10 \mathrm{~Hz}, 1 \mathrm{H}) ; 3.59(\mathrm{dd}, J=5,10 \mathrm{~Hz}, 1 \mathrm{H}) ; 3.14$ (burried $\mathrm{m}, 1 \mathrm{H}) ; 3.08(\mathrm{dd}, J=8$, $15.5 \mathrm{~Hz}, 1 \mathrm{H}) ; 3.03(\mathrm{dd}, J=4,13.5 \mathrm{~Hz}, 1 \mathrm{H}) ; 2.98-2.93(\mathrm{~m}, 1 \mathrm{H}) ; 2.90(\mathrm{~d}, J=6.5 \mathrm{~Hz}, 2 \mathrm{H}) ; 2.75(\mathrm{dd}, J$ $=10,13.5 \mathrm{~Hz}, 1 \mathrm{H}) ; 2.45$ (burried $\mathrm{m}, 1 \mathrm{H}) ; 2.00-1.95(\mathrm{~m}, 1 \mathrm{H}) ; 1.79-1.73(\mathrm{~m}, 1 \mathrm{H}) ; 1.12-0.99(\mathrm{~m}$, 21H). ${ }^{13} \mathrm{C}$ NMR (100 MHz, $\left.\mathbf{C D C l}_{3}\right): \delta$ 172.5, 164.5, 162.8, 162.4, 157.1, 156.8, 143.3, 137.1, 136.6, 129.6, 129.3, 129.0, 128.8, 127.3, 127.0, 121.7, 114.3, 94.6 64.9, 55.4, 53.4, 52.3, 52.0, 50.7, 50.5, 37.8, 37.7, 29.0, 23.8, 18.1, 12.0. IR (neat): 3365, 2943, 2864 (all w); 1727, 1647, 1540, 1222 (all s). HRMS (ESI+): Calc. for $\mathrm{C}_{44} \mathrm{H}_{60} \mathrm{~N}_{7} \mathrm{O}_{9} \mathrm{Si}(\mathrm{m} / \mathrm{z})$ 858.4222; found $(\mathrm{m} / \mathrm{z}) 858.4186$. 
Macrocylcic triazole $20 \mathrm{~b}(5 R, 9 R, 17 S)$. The triazole $20 \mathrm{~b}$ was prepared according to general procedure $\mathrm{E}$ from $19 \mathrm{~b}(83 \mathrm{mg}, 97 \mu \mathrm{mol})$ and purified on silica eluting with 1:1 Hexanes : EtOAc $\rightarrow$ EtOAc to give the macrocycle as a white solid $(48 \mathrm{mg}, 58 \%) .(R, R, S)[\alpha]^{25} \mathrm{D}=-7.4(\mathrm{c}=0.58$, $\mathrm{CH}_{2} \mathrm{Cl}_{2}$ ). ${ }^{1} \mathrm{H}$ NMR (500 MHz, DMSO- $\left.\boldsymbol{d}_{6}, 353 \mathrm{~K}\right): \delta 8.24$ (br s, $\left.1 \mathrm{H}\right) ; 7.97$ (br s, $1 \mathrm{H}) ; 7.73(\mathrm{~s}, 1 \mathrm{H}) ; 7.32-7.14(\mathrm{~m}, 10 \mathrm{H}) ; 6.25(\mathrm{~d}, J=8 \mathrm{~Hz}, 1 \mathrm{H}) ; 5.72(\mathrm{~d}, J=8.5$ $\mathrm{Hz}, 1 \mathrm{H}) ; 4.68(\mathrm{dd}, J=8.5,14 \mathrm{~Hz}, 1 \mathrm{H}) ; 4.55(\mathrm{dd}, J=6,15 \mathrm{~Hz}, 1 \mathrm{H}) ; 4.51-4.46$

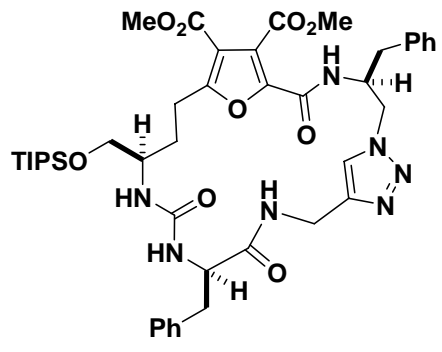
$(\mathrm{m}, 3 \mathrm{H}) ; 4.28(\mathrm{dd}, J=8.5,14 \mathrm{~Hz}, 1 \mathrm{H}) ; 4.10(\mathrm{dd}, J=3.5,15 \mathrm{~Hz}, 1 \mathrm{H}) ; 3.77(\mathrm{~s}, 3 \mathrm{H}) ; 3.74(\mathrm{~s}, 3 \mathrm{H}) ; 3.74-$ 3.71 (burried $\mathrm{m}, 1 \mathrm{H}) ; 3.67(\mathrm{dd}, J=4,10 \mathrm{~Hz}, 1 \mathrm{H}) ; 3.52(\mathrm{dd}, J=5,10 \mathrm{~Hz}, 1 \mathrm{H}) ; 3.41$ (br s, $1 \mathrm{H}) ; 3.18$ (ddd, $J=6,9.5,15 \mathrm{~Hz}, 1 \mathrm{H}) ; 3.00(\mathrm{dd}, J=5.5,14.5 \mathrm{~Hz}, 1 \mathrm{H}) ; 2.93($ app t, $J=6 \mathrm{~Hz}, 2 \mathrm{H}) ; 2.96-2.85$ (burried $\mathrm{m}, 2 \mathrm{H}) ; 2.76(\mathrm{dd}, J=9,14 \mathrm{~Hz}, 1 \mathrm{H}) ; 2.03-1.97(\mathrm{~m}, 1 \mathrm{H}) ; 1.82-1.78(\mathrm{~m}, 1 \mathrm{H}) ; 1.11-1.02(\mathrm{~m}$, 21H). ${ }^{13} \mathrm{C}$ NMR (100 MHz, $\left.\mathbf{C D C l}_{3}\right): \delta 172.8,164.2,162.9,162.5,157.1,156.8,142.8,137.2,136.9$, 129.4, 129.0, 128.9, 127.2, 127.1, 122.5, 114.4, 64.6, 55.5, 53.3, 52.3, 51.7, 51.5, 37.7, 37.5, 29.6, 24.2, 18.1, 12.0. IR (neat): 3306, 2943, 2864 (all w); 1726, 1650, 1530, 1221 (all s). HRMS (ESI+): Calc. for $\mathrm{C}_{44} \mathrm{H}_{60} \mathrm{~N}_{7} \mathrm{O}_{9} \mathrm{Si}(\mathrm{m} / \mathrm{z})$ 858.4222; found $(\mathrm{m} / \mathrm{z}) 858.4211$.

Macrocylcic triazole 20c $(5 R, 9 S, 17 R)$. The triazole 20c was prepared according to general procedure $\mathrm{E}$ from $19 \mathrm{c}(66 \mathrm{mg}, 77 \mu \mathrm{mol})$ and purified on silica eluting with 1:1 Hexanes : EtOAc $\rightarrow$ EtOAc to give the macrocycle as a white solid $(21 \mathrm{mg}, 32 \%) .(\boldsymbol{R}, S, R)[\alpha]^{25} \mathrm{D}=+50.0$ $\left(\mathrm{c}=0.40, \mathrm{CH}_{2} \mathrm{Cl}_{2}\right) .{ }^{1} \mathrm{H}$ NMR $\left(500 \mathrm{MHz}\right.$, DMSO- $\left.d_{6}, 353 \mathrm{~K}\right): \delta 7.90(\mathrm{~d}, J=$ $7 \mathrm{~Hz}, 1 \mathrm{H}) ; 7.86(\mathrm{~s}, 1 \mathrm{H}) ; 7.32-7.19(\mathrm{~m}, 10 \mathrm{H}) ; 6.17(\mathrm{~d}, J=6.5 \mathrm{~Hz}, 1 \mathrm{H}) ; 5.76$ $(\mathrm{d}, J=8 \mathrm{~Hz}, 1 \mathrm{H}) ; 5.56(\mathrm{~d}, J=5.5 \mathrm{~Hz}, 2 \mathrm{H}) ; 4.54(\mathrm{dt}, J=6.5,14.5 \mathrm{~Hz}, 1 \mathrm{H})$;

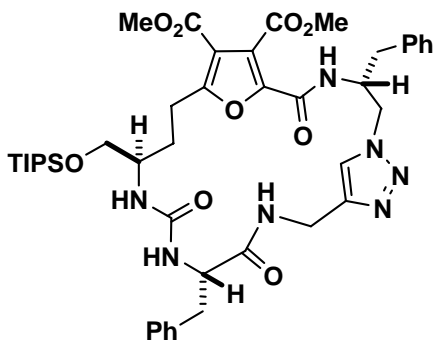
$4.55(\operatorname{app~q}, J=5.5 \mathrm{~Hz}, 1 \mathrm{H}) ; 4.08(\mathrm{~d}, J=14 \mathrm{~Hz}, 1 \mathrm{H}) ; 4.00-3.70($ br s, $1 \mathrm{H}) ; 3.76(\mathrm{~s}, 3 \mathrm{H}) ; 3.75(\mathrm{~s}, 3 \mathrm{H})$; 3.75-3.72 (burried m, $1 \mathrm{H}) ; 3.64(\mathrm{dd}, J=4,10 \mathrm{~Hz}, 1 \mathrm{H}) ; 2.99-2.91(\mathrm{~m}, 4 \mathrm{H}) ; 2.00-1.98(\mathrm{~m}, 1 \mathrm{H}) ; 1.79-$ $1.76(\mathrm{~m}, 1 \mathrm{H}) ; 1.12-1.01 \quad(\mathrm{~m}, 21 \mathrm{H}) .{ }^{13} \mathrm{C}$ NMR (100 $\left.\mathbf{M H z}, \mathbf{C D C l}_{3}\right): \delta 172.5,164.4,162.8,162.7$, 157.4, 157.0, 138.0, 136.9, 129.4, 129.3, 129.1, 128.9, 127.2, 127.0, 114.3, 65.1, 57.4, 53.4, 52.4, 51.4, 51.1, 37.1, 36.8, 35.3, 29.2, 24.1, 18.2, 12.1. IR (neat): 3382, 2944, 2865 (all m); 1727, 1667, 1447, 1224 (all s). HRMS (ESI+): Calc. for $\mathrm{C}_{44} \mathrm{H}_{60} \mathrm{~N}_{7} \mathrm{O}_{9} \mathrm{Si}(\mathrm{m} / \mathrm{z})$ 858.4222; found $(\mathrm{m} / \mathrm{z}) 858.4210$.

Macrocylcic triazole $20 \mathrm{~d}(5 R, 9 S, 17 S)$. The triazole $20 \mathrm{~d}$ was prepared according to general procedure E from $19 \mathrm{~d}(79 \mathrm{mg}, 92$ $\mu \mathrm{mol})$ and purified on silica eluting with 1:1 Hexanes : EtOAc $\rightarrow$ EtOAc to give the macrocycle as a white solid (55 $\mathrm{mg}, 70 \%)$. $(\boldsymbol{R}, S, S)[\alpha]^{25} \mathrm{D}=-31.0 \quad\left(\mathrm{c}=0.76, \mathrm{CH}_{2} \mathrm{Cl}_{2}\right) .{ }^{1} \mathrm{H}$ NMR $(500 \mathbf{~ M H z}$, DMSO- $\left.d_{6,}, 343 \mathrm{~K}\right): \delta 8.17(\mathrm{~d}, J=7.5 \mathrm{~Hz}, 1 \mathrm{H}) ; 7.99(\mathrm{br} \mathrm{s}, 1 \mathrm{H}) ; 7.82(\mathrm{~s}$, $1 \mathrm{H}) ; 7.32-7.18(\mathrm{~m}, 10 \mathrm{H}) ; 6.14(\mathrm{~d}, J=7 \mathrm{~Hz}, 1 \mathrm{H}) ; 5.92(\mathrm{~d}, J=7.5 \mathrm{~Hz}$,

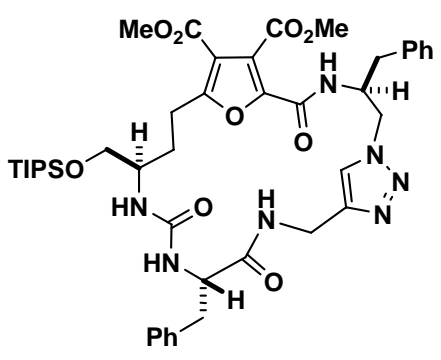
$1 \mathrm{H}) ; 4.64(\mathrm{dd}, J=8,13.5 \mathrm{~Hz}, 1 \mathrm{H}) ; 4.52-4.46(\mathrm{~m}, 2 \mathrm{H}) ; 4.38(\mathrm{dd}, J=6,15.5 \mathrm{~Hz}, 1 \mathrm{H}) ; 4.29(\mathrm{dd}, J=$ $5.5,15.5 \mathrm{~Hz}, 1 \mathrm{H}) ; 4.13$ (br s, 1H); 3.78-3.74 (burried m, 2H); 3.76 (s, 3H); 3.75 (s, 3H); 3.61 (dd, J $=5.5,9.5 \mathrm{~Hz}, 1 \mathrm{H}) ; 3.45(\mathrm{br} \mathrm{m}, 1 \mathrm{H}) ; 3.15(\mathrm{dd}, J=7,15 \mathrm{~Hz}, 1 \mathrm{H}) ; 3.05(\mathrm{dd}, J=4,14 \mathrm{~Hz}, 1 \mathrm{H}) ; 2.95-$ $2.81(\mathrm{~m}, 5 \mathrm{H}) ; 2.06(\mathrm{dt}, J=7,14 \mathrm{~Hz}, 1 \mathrm{H}) ; 1.94(\mathrm{dt}, J=6.5,14 \mathrm{~Hz}, 1 \mathrm{H}) ; 1.08-0.97(\mathrm{~m}, 21 \mathrm{H}) .{ }^{13} \mathrm{C}$ NMR (100 MHz, $\left.\mathrm{CDCl}_{3}\right)$ : $\delta 173.2,164.5,162.9,162.5,157.3,156.8,143.4,137.1,136.8,129.3$, 129.2, 129.0, 128.9, 128.3, 127.2, 121.9, 114.3, 65.1, 56.5, 53.3, 52.3, 51.2, 50.5, 37.6, 37.1, 29.3, 23.5, 18.2, 12.0. IR (neat): 3369, 2944, 2865 (all m); 1726, 1652, 1539, 1445, 1223 (all s). HRMS (ESI+): Calc. for $\mathrm{C}_{44} \mathrm{H}_{60} \mathrm{~N}_{7} \mathrm{O}_{9} \mathrm{Si}(\mathrm{m} / \mathrm{z})$ 858.4222; found $(\mathrm{m} / \mathrm{z}) 858.4203$. 
VII General procedure F for the acyclic cycloaddition reactions:

Linear Triazole 13a. A solution of the morpholino-azide $(0.10 \mathrm{~g}, 0.13$ $\mathrm{mmol})$ in PhMe $(20 \mathrm{ml})$ was added $\mathrm{CuI}(9.7 \mathrm{mg}, 65 \mu \mathrm{mol})$ and $i \mathrm{Pr}_{2} \mathrm{NEt}$ (335 mg, $2.6 \mathrm{mmol}$ ). Phenylacetylene (63 $\mu \mathrm{l}, 0.60 \mathrm{mmol})$ was then added and the mixture stirred for $12 \mathrm{~h}$.. The mixture was concentrated and purified on silica eluting with $9: 1$ EtOAc: hexanes 10 give 13a as a white solid (100 mg, 90\%) $0.1 \mathrm{~g}$ (yield $90 \%$ ). ${ }^{1} \mathrm{H}$ NMR (500 MHz, $\left.\mathrm{CDCl}_{3}\right)$ : $\delta 7.81(\mathrm{~s}, 1 \mathrm{H}), 7.74(\mathrm{~d}, J=7 \mathrm{~Hz}, 2 \mathrm{H}), 7.37-7.22(\mathrm{~m}, 8 \mathrm{H}), 4.89(\mathrm{~d}, J$ $=7.5 \mathrm{~Hz}, 1 \mathrm{H}), 4.71(\mathrm{~d}, J=9.5 \mathrm{~Hz}, 1 \mathrm{H}), 4.53(\mathrm{dd}, J=5,12.5 \mathrm{~Hz}, 1 \mathrm{H}), 4.49$ $4.42(\mathrm{~m}, 2 \mathrm{H}), 3.90(\mathrm{~s}, 3 \mathrm{H}), 3.80-3.76$ (buried $\mathrm{m}, 1 \mathrm{H}), 3.76(\mathrm{~s}, 3 \mathrm{H}), 3.70$

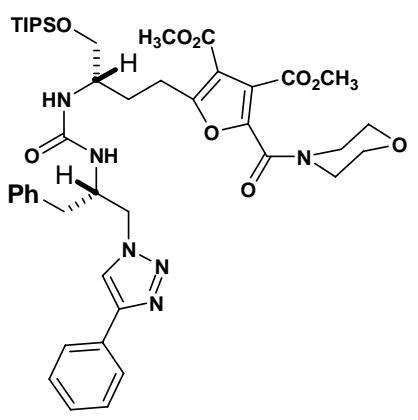
$(\mathrm{dd}, J=2.5,10 \mathrm{~Hz}, 8 \mathrm{H}), 3.66$ (buried dd, $J=3,10 \mathrm{~Hz}, 1 \mathrm{H}), 3.62(\mathrm{dd}, J=5,9.5 \mathrm{~Hz}, 1 \mathrm{H}), 2.98-2.81$ $(\mathrm{m}, 3 \mathrm{H}), 2.81(\mathrm{dd}, J=7,14 \mathrm{~Hz}, 1 \mathrm{H}), 1.92-1.85(\mathrm{~m}, 1 \mathrm{H}), 1.83-1.75(\mathrm{~m}, 1 \mathrm{H}), 1.01-0.99(\mathrm{~m}, 21 \mathrm{H}) .{ }^{13} \mathrm{C}$ NMR (125 MHz, $\left.\mathrm{CDCl}_{3}\right): \delta 163.4,162.8,162.3,157.6,156.9,147.4,144.3,136.9,130.2,129.3$, 128.7, 128.6, 128.1, 126.8, 125.4, 122.9, 121.1, 113.2, 66.6, 65.4, 52.8, 52.7, 52.4, 52.0, 52.0, 51.2, 51.0, 38.4, 30.0, 24.4, 17.9, 11.7. IR (Dep. $\left.\mathrm{CDCl}_{3}\right):$ 3380, 2944, 2865, 1726, 1652, 1634, 1557, 1496, 1446, 1300, 1279, 1224, 1118, 1077, 1021. HRMS (ESI+) Calc. for $\mathrm{C}_{44} \mathrm{H}_{60} \mathrm{~N}_{6} \mathrm{O} 9 \mathrm{Si}(\mathrm{m} / z)$ : 845.4269. Found $(m / z): 845.4261$.

Linear Triazole 13b. $\mathbf{1 3 b}$ was prepared according to General Procedure $\mathrm{F}$ with the azide $(30 \mathrm{mg}, 40 \mu \mathrm{mol})$ and 2-ethynylpyridine $(14.3 \mu \mathrm{l}, 0.14 \mathrm{mmol})$. The crude product was purified on silica eluting with 9: 1 EtOAc: hexanes to afford the product as colorless oil (26 mg, 62\%). ${ }^{1} \mathrm{HNMR}\left(400 \mathrm{MHz}, \mathrm{CDCl}_{3}\right) \delta 8.47(\mathrm{~m}, 1 \mathrm{H}), 8.17(\mathrm{~m}, 1 \mathrm{H}), 8.04(\mathrm{~m}$, $1 \mathrm{H}), 7.73(\mathrm{~m}, 1 \mathrm{H}), 7.32-7.28(\mathrm{~m}, 1 \mathrm{H}), 7.26-7.22(\mathrm{~m}, 5 \mathrm{H}), 4.86(\mathrm{~m}, 1 \mathrm{H})$, $4.74(\mathrm{~d}, J=7.6 \mathrm{~Hz}, 1 \mathrm{H}), 3.89(\mathrm{~s}, 3 \mathrm{H}), 3.80$ (buried $\mathrm{m}, 1 \mathrm{H}), 3.76(\mathrm{~s}, 3 \mathrm{H})$, $3.71(\mathrm{~m}, 8 \mathrm{H}), 3.68$ (buried dd, $J=4,18.4 \mathrm{~Hz}, 1 \mathrm{H}), 3.62(\mathrm{dd}, J=4,10 \mathrm{~Hz}$,

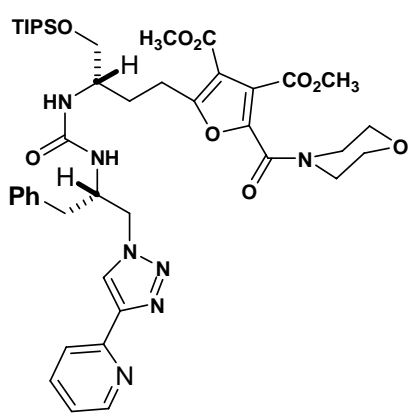
$1 \mathrm{H}), 3.00-2.98(\mathrm{~m}, 1 \mathrm{H}), 2.90-2.83(\mathrm{~m}, 2 \mathrm{H}), 1.92-1.80(\mathrm{~m}, 3 \mathrm{H}), 1.05-1.00$ $(\mathrm{m}, 21 \mathrm{H})$. MS (APCI+) Calc. for $\mathrm{C}_{43} \mathrm{H}_{59} \mathrm{~N}_{7} \mathrm{O}_{9} \mathrm{Si}(\mathrm{m} / \mathrm{z}): 845.41$. Found $(\mathrm{m} / \mathrm{z}): 846.74$.

Linear Triazole 13c. 13c was prepared according to General Procedure F with the azide $(30 \mathrm{mg}, 40 \mu \mathrm{mol})$ and 1ethynylcyclopentanol (16 $\mu \mathrm{l}, 0.14 \mathrm{mmol})$. The crude product was purified on silica eluting with 9.5: 0.5 EtOAc: $\mathrm{iPrOH}$ to afford the product as a white solid (26 mg, 75\%). $\left.{ }^{1} \mathrm{H} \mathrm{NMR} \mathrm{(500} \mathrm{MHz,} \mathrm{CDCl}_{3}\right): \delta$ 7.48 (br s, 1H), 7.26-7.19 (m, 5H), 5.15 (d, J = 7.5 Hz), 4.97 (d, J = 9 Hz, $1 \mathrm{H}), 4.43-4.33(\mathrm{~m}, 3 \mathrm{H}), 3,88(\mathrm{~s}, 3 \mathrm{H}), 3.79(\mathrm{~s}, 3 \mathrm{H}), 3.79-3.78$ (buried m, $1 \mathrm{H}), 3.70-3.66(\mathrm{~m}, 8 \mathrm{H}), 3.60(\mathrm{dd}, J=5.5,10 \mathrm{~Hz}, 1 \mathrm{H}), 3.16$ (br s, $1 \mathrm{H})$,

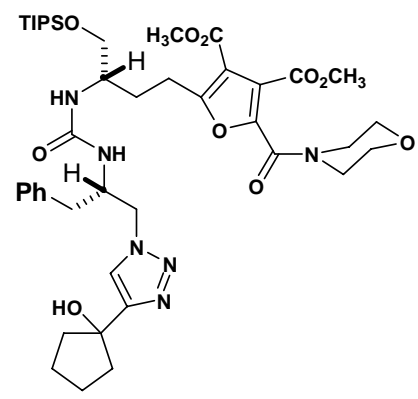
$3.07(\mathrm{ddd}, J=6.5,9.5,15 \mathrm{~Hz}, 1 \mathrm{H}), 2.97(\mathrm{ddd}, J=6.5,8.5,15 \mathrm{~Hz}, 1 \mathrm{H}), 2.86(\mathrm{dd}, J=7.5,15 \mathrm{~Hz}$, $1 \mathrm{H}), 2.77(\mathrm{dd}, J=7.5,15 \mathrm{~Hz}, 1 \mathrm{H}), 2.00-1.92(\mathrm{~m}, 8 \mathrm{H}), 1.81-1.75(\mathrm{~m}, 2 \mathrm{H}), 1.05-1.01(\mathrm{~m}, 21 \mathrm{H}) . \mathrm{MS}$ (APCI+) Calc. for $\mathrm{C}_{43} \mathrm{H}_{64} \mathrm{~N}_{6} \mathrm{O}_{10} \mathrm{Si}(\mathrm{m} / \mathrm{z}): 853.44$. Found $(\mathrm{m} / \mathrm{z}): 853.76$. 
RELI284-1

Pulse Sequence: s2pul

Solvent: $\operatorname{CDC} 13$

Temp. $23.0 \mathrm{C} / 296.1 \mathrm{~K}$
Mercury-400BB "mercury400b"

Pulse 77.1 degrees

Acq. time 2.731 sec

Width $5998.8 \mathrm{~Hz}$

OBSERVE H1, 399.8366318 $\mathrm{MHZ}$

DATA PROCESSIN

Line broadening $0.1 \mathrm{~Hz}$

total tine 1 inin, 2 sec
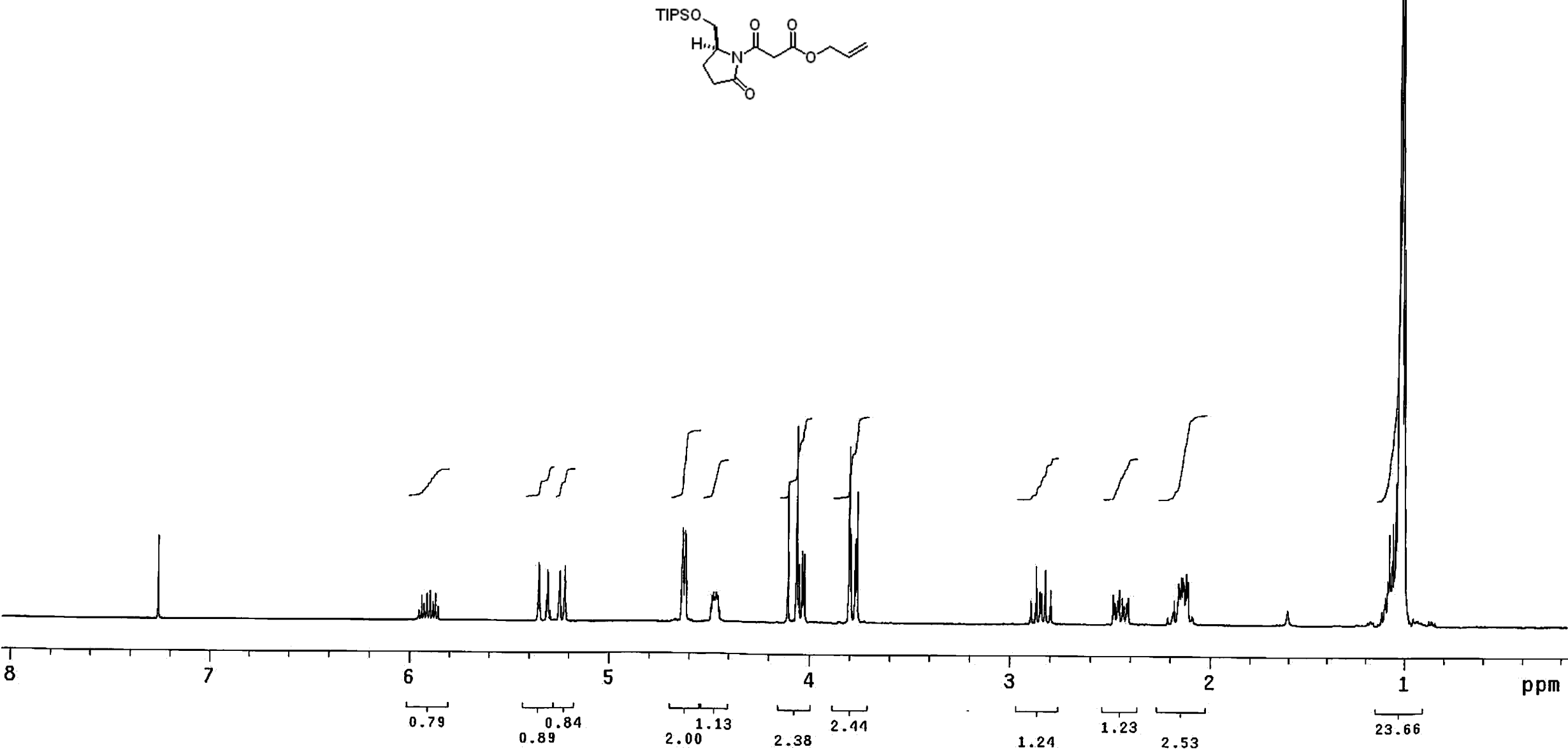


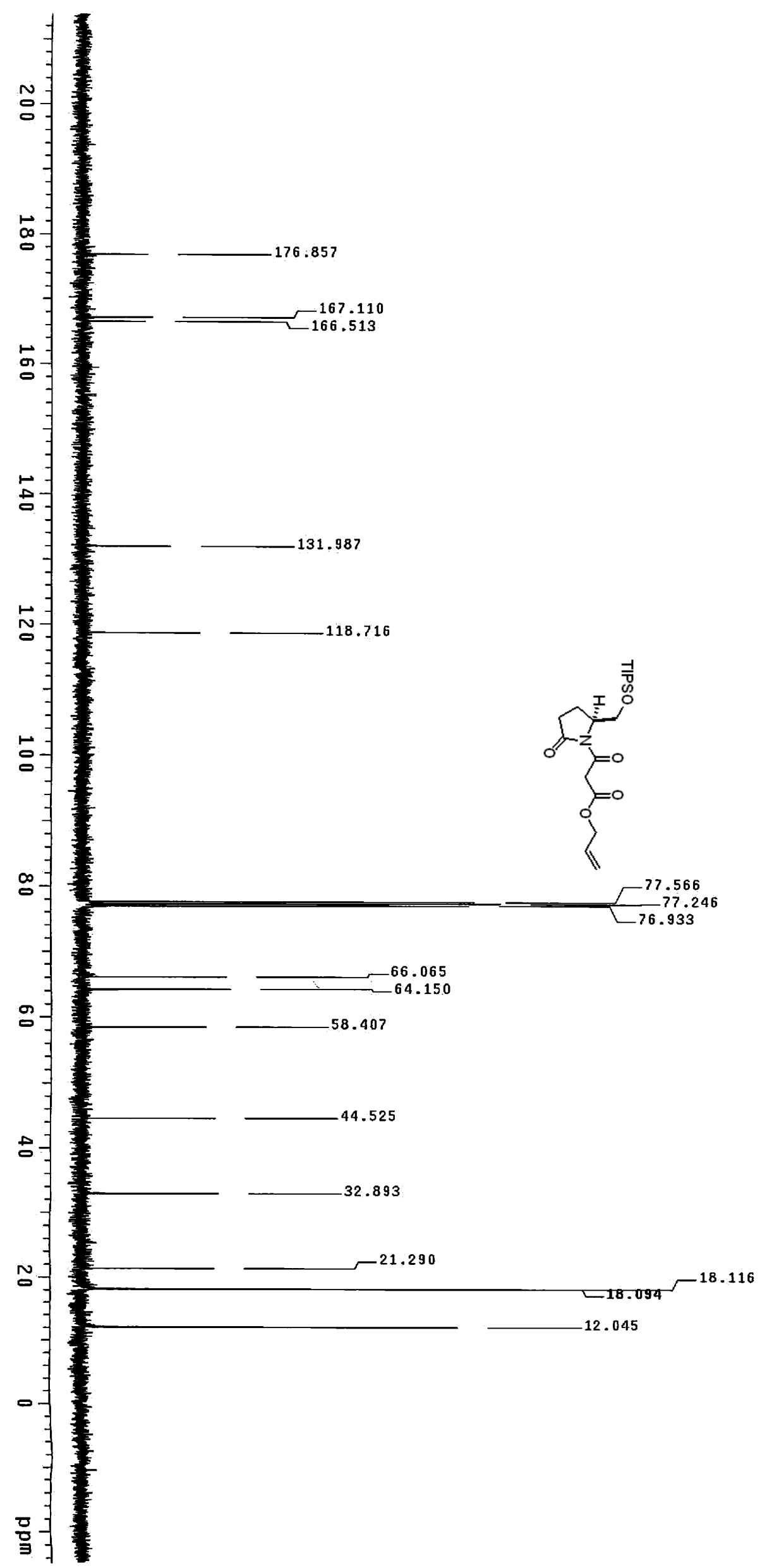




\section{RELI291-1}

Pulse Sequence: s2pul

Solvent: $\operatorname{CDC} 13$

Temp. $23.0 \mathrm{C} / 296.1 \mathrm{~K}$
Mercury-400BB

Pulse 77.1 degrees

widith $5998.8 \mathrm{~Hz}$

16 repetitions
OBSERVE H1 $399.8366318 \mathrm{MHZ}$

DATA PROCESSING

FT size 327ening $0.1 \mathrm{~Hz}$

Total time $1 \mathrm{~min}, 2 \mathrm{sec}$
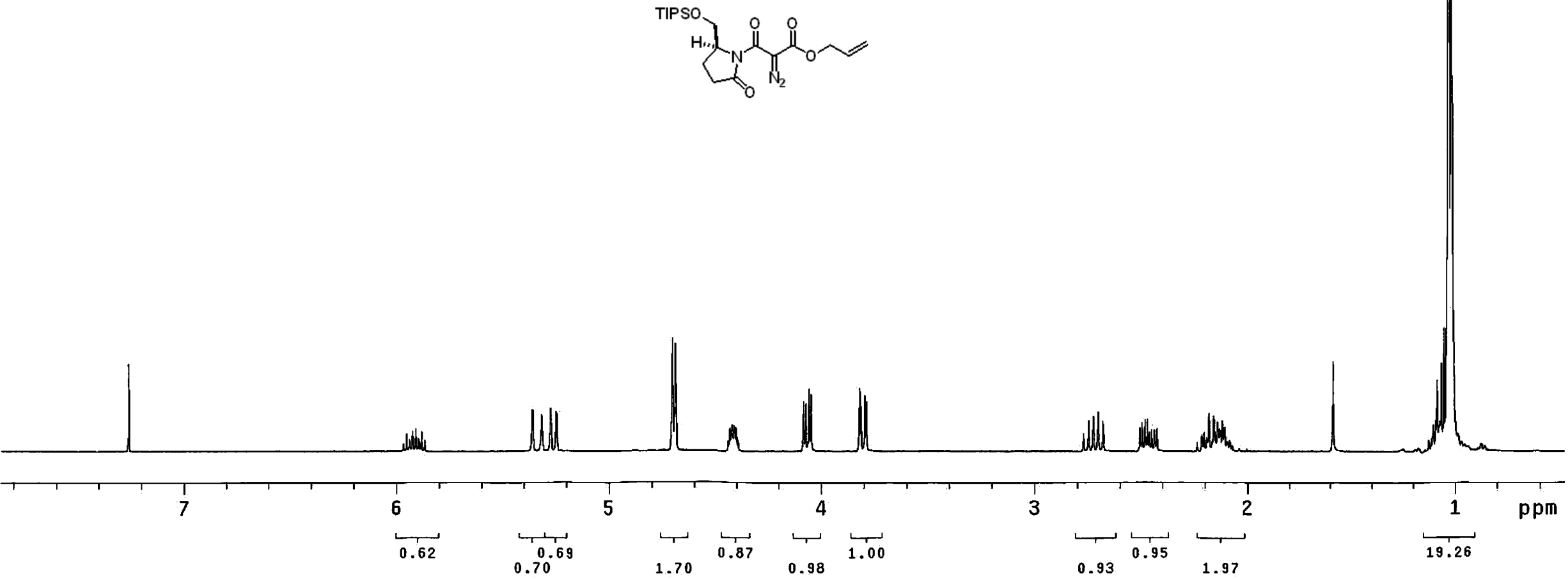
Pulse Sequence: s2pul

Solvent: $\mathrm{CDC}_{13}$

Temp. $23.0 \mathrm{C} / 296.1 \mathrm{~K}$
Mercury-400BB "mercury400b"

Re lax. de lay $1.300 \mathrm{sec}$

Pulse 36.7 degrees

Acq. time $1.311 \mathrm{se}$

176 repetitions

OBSERVE C13, 100.5389823 MHZ

DECOUPLE H1, $399.8385865 \mathrm{MHZ}$

Power 47 dB

cont inuous iy on

DATA PROCESSING

Line broadening $1.0 \mathrm{~Hz}$

FT size $65536 \mathrm{hr}, 2 \mathrm{~min}, 4 \mathrm{sec}$

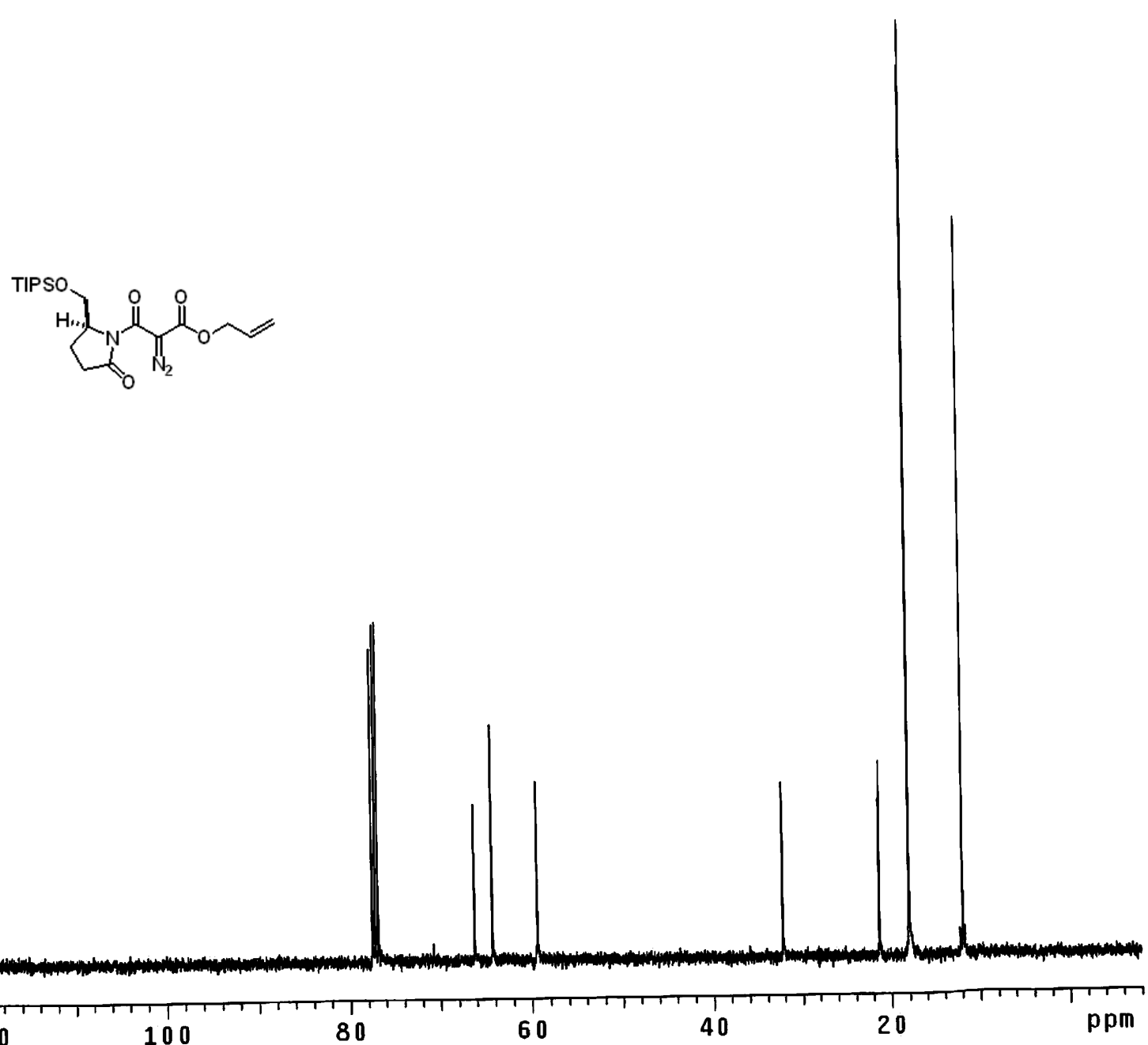




\section{RELI285}

Pulse Sequence: s2pul

Solvent: $\operatorname{CoC} 13$

Mercury-40OBB "mercury400b"

Pulse 77.1 degrees

Width $5998.8 \mathrm{~Hz}$

16 repetitions

OBSERVE H1, $399.8366318 \mathrm{MHZ}$

Line broadening $0.1 \mathrm{~Hz}$

FT size 32768
Total time $1 \mathrm{~min}, 2 \mathrm{sec}$
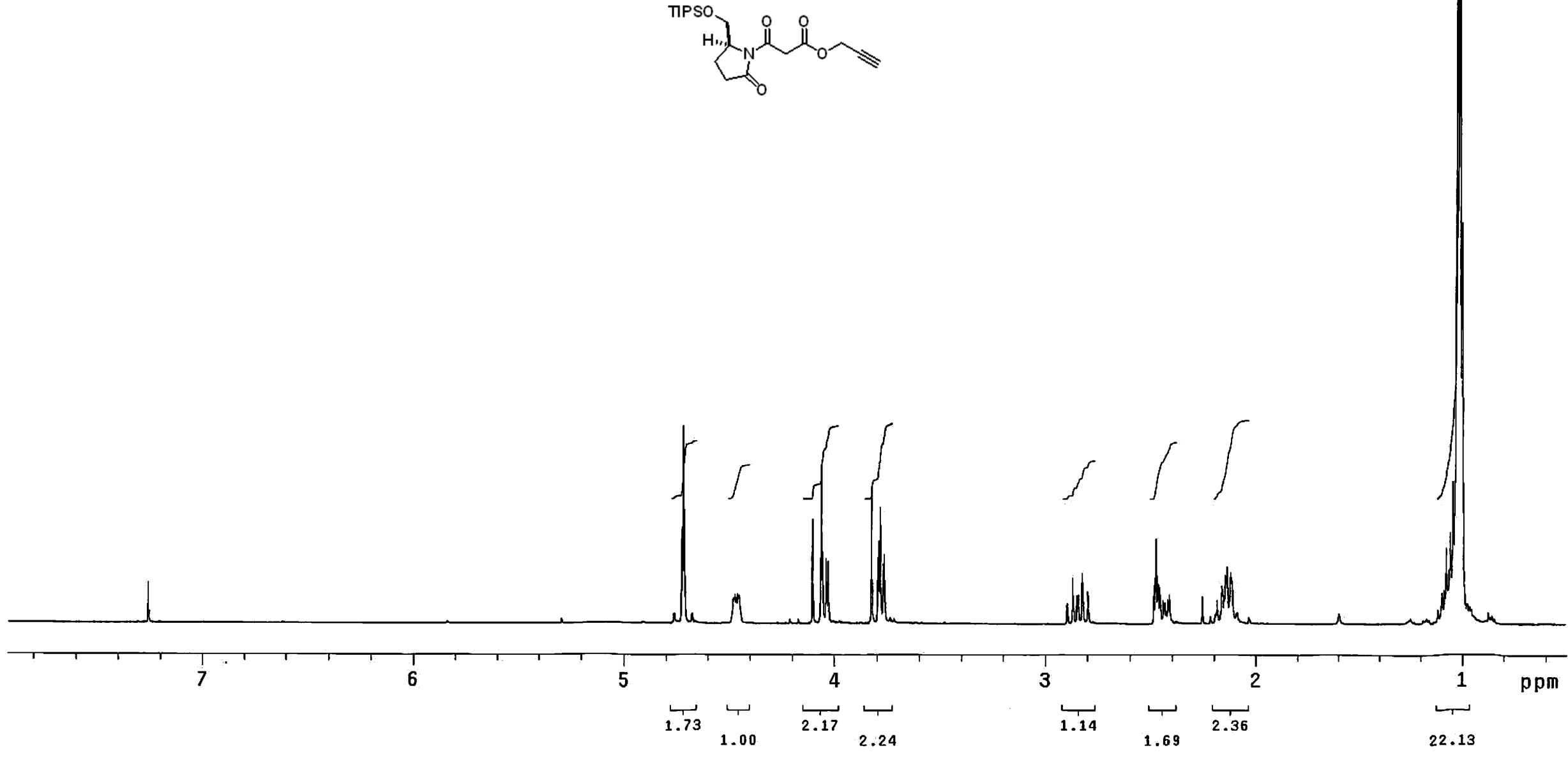


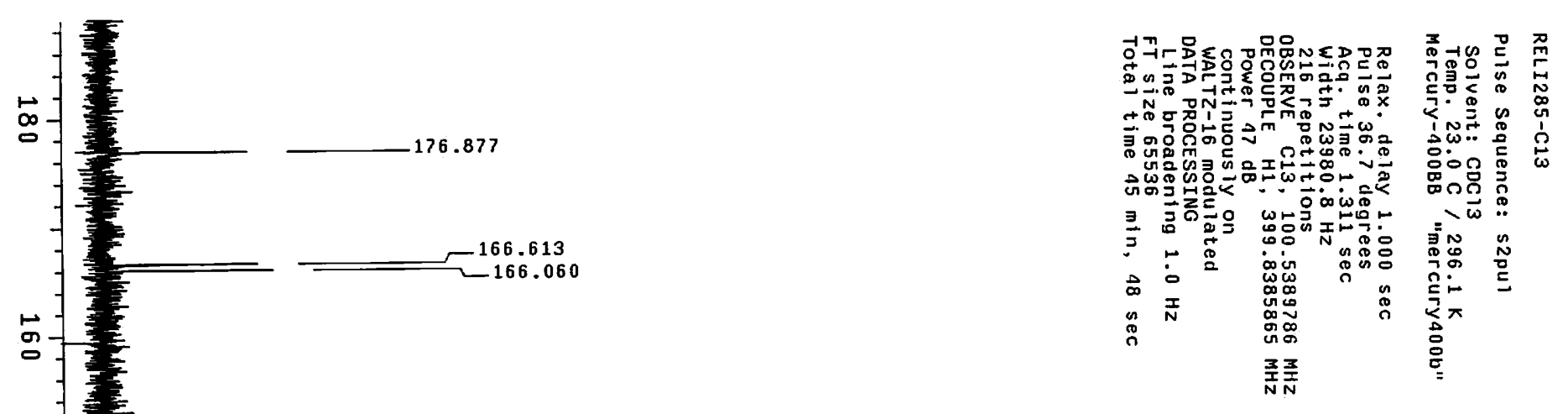

N

N

品-

ㅇ.

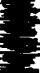

$\frac{7}{3}$

$\infty$ 接
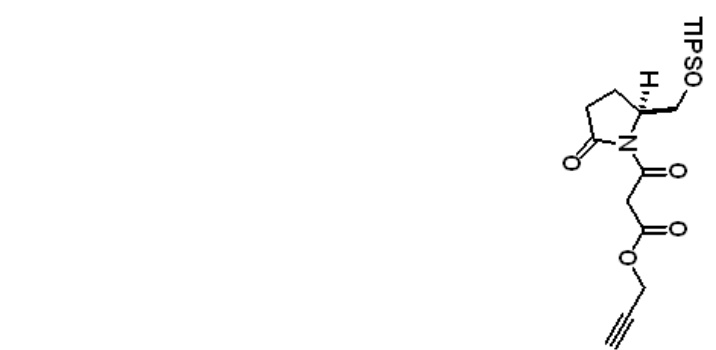

64.113

o
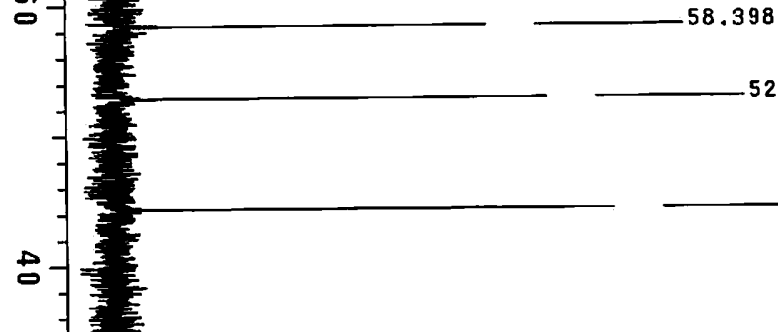

52.851

44.320

N

21.303

777.543

$-77.230$

76.910

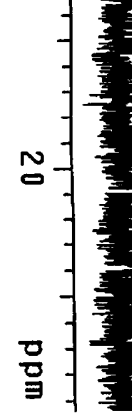


RELI285-diazo

Pulse Sequence: s2pur

Solvent: $\operatorname{CDC}_{13}$

Temp. $22.0 \mathrm{C} / 295.1 \mathrm{~K}$

Relax. delay $0.120 \mathrm{sec}$

Pcise time d agrees

width $5995.2 \mathrm{HZ}$

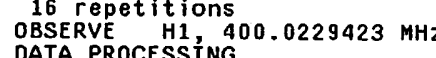

FT Size 32768 ing $0.1 \mathrm{~Hz}$

Total time $1 \mathrm{~min}, 4 \mathrm{sec}$

TIPSO
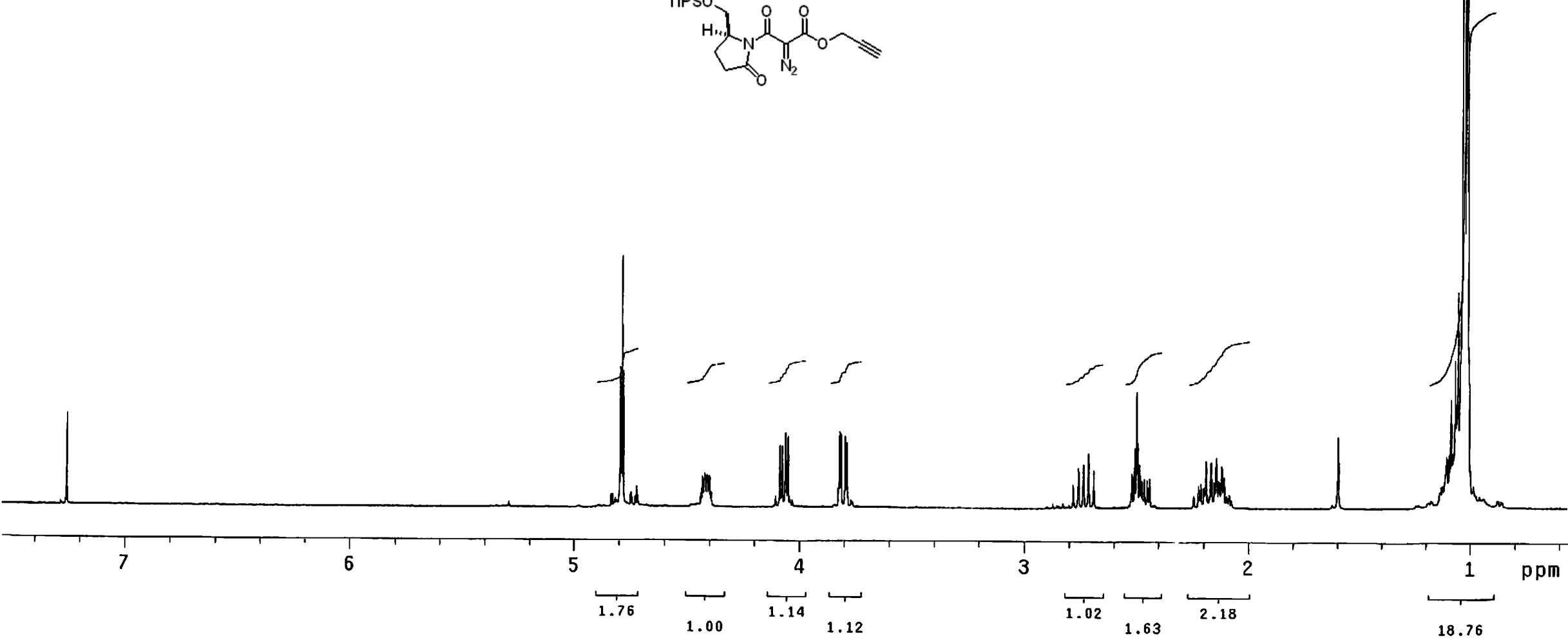


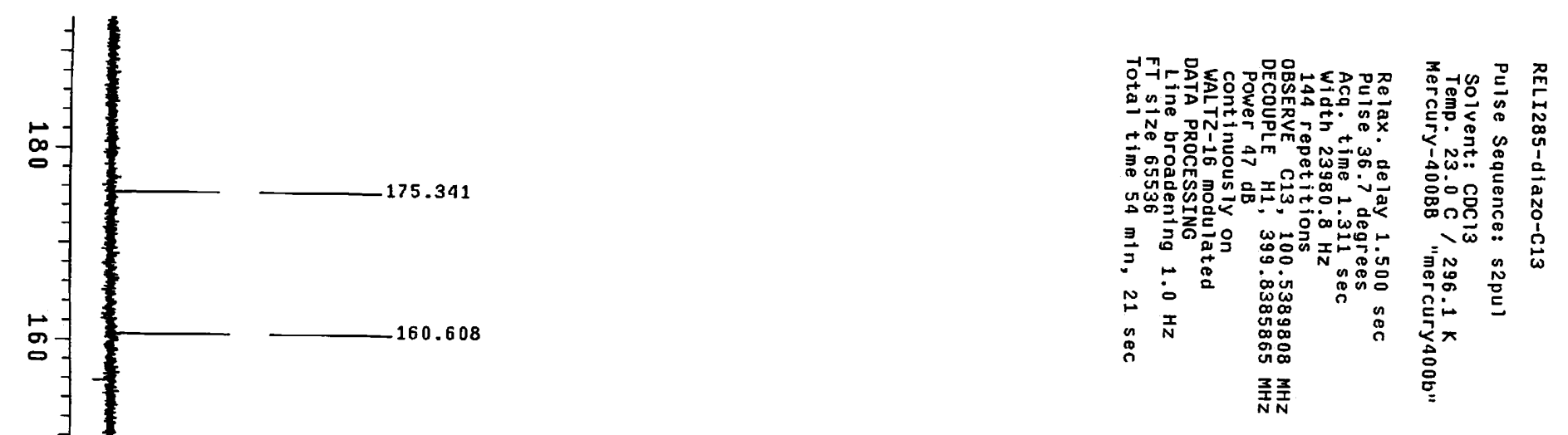

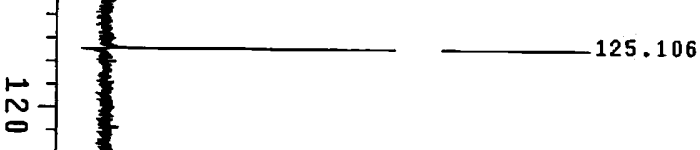

車

$\stackrel{\leftrightarrow}{\circ}$

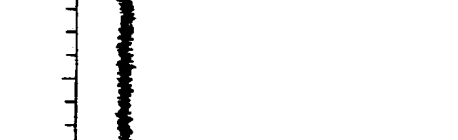

怘

$\infty$ 南事 77.550

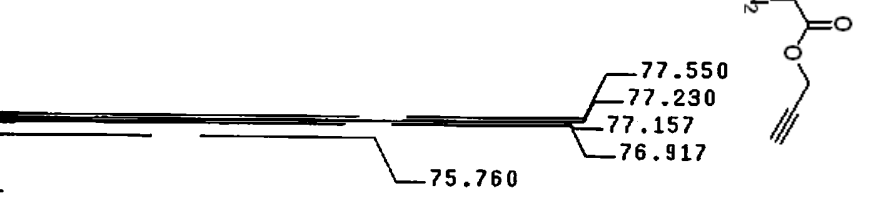

$\begin{aligned}-1 & = \\ -1 & = \\ 8-1 & =1\end{aligned}$

o
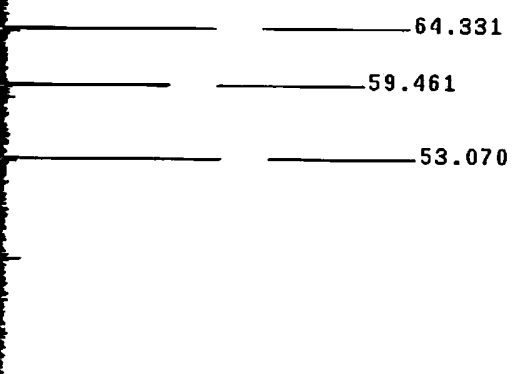

1

32.222

음

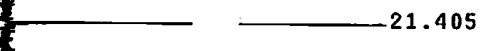

53.070

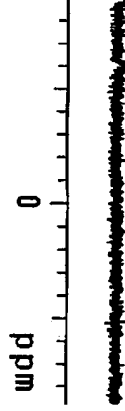

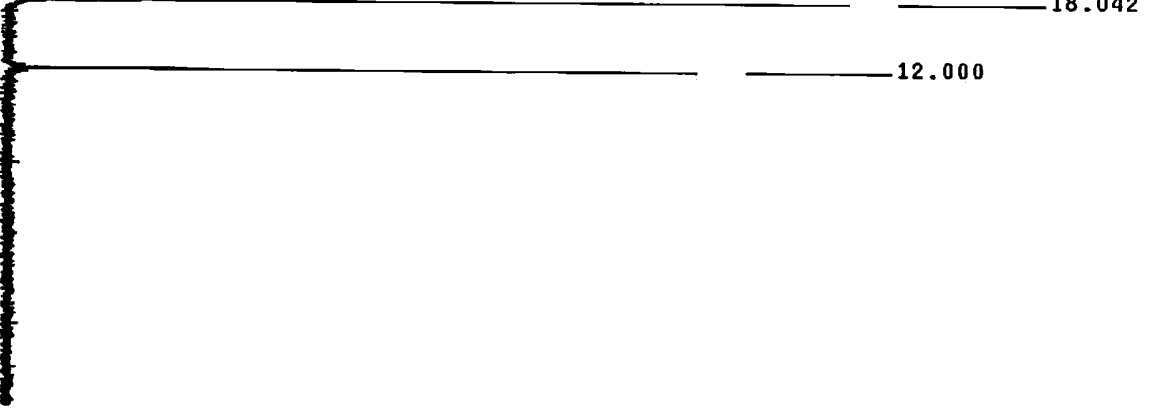


RELI 290

Pulse Sequence: s2pul

Solvent: $\mathrm{CDC}_{13}$

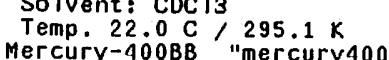

Relax. de lay $0.120 \mathrm{sec}$

Pulse 61.3 degrees

Acq. time $2.731 \mathrm{sec}$

Width 595.2 H

OBSERVE H1, $400.0229423 \mathrm{MHZ}$

DATA PROCESSING

Line broadening $0.1 \mathrm{~Hz}$

Total time $7 \mathrm{~min}, 28 \mathrm{sec}$

TIPSO
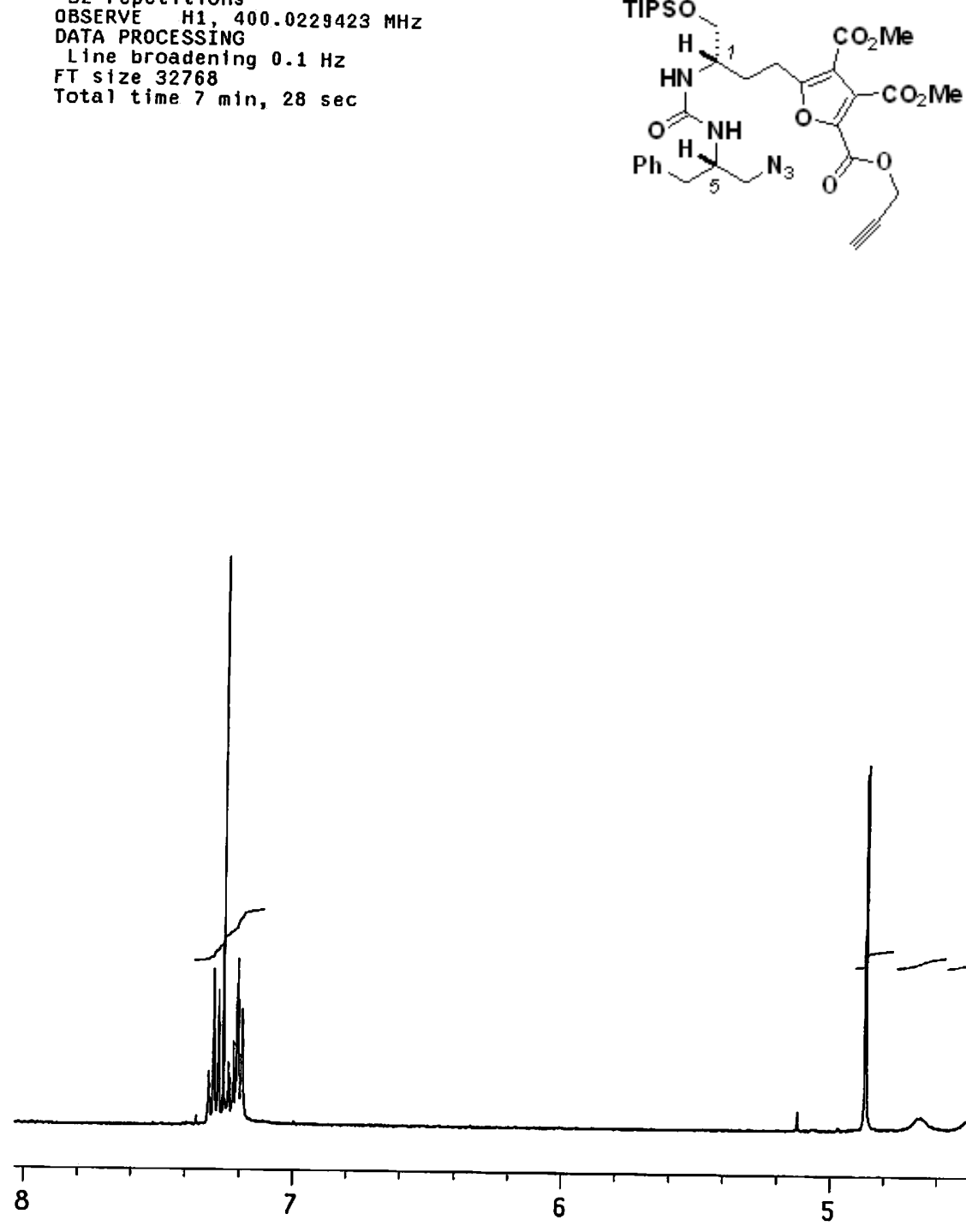

5

3

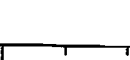

1 


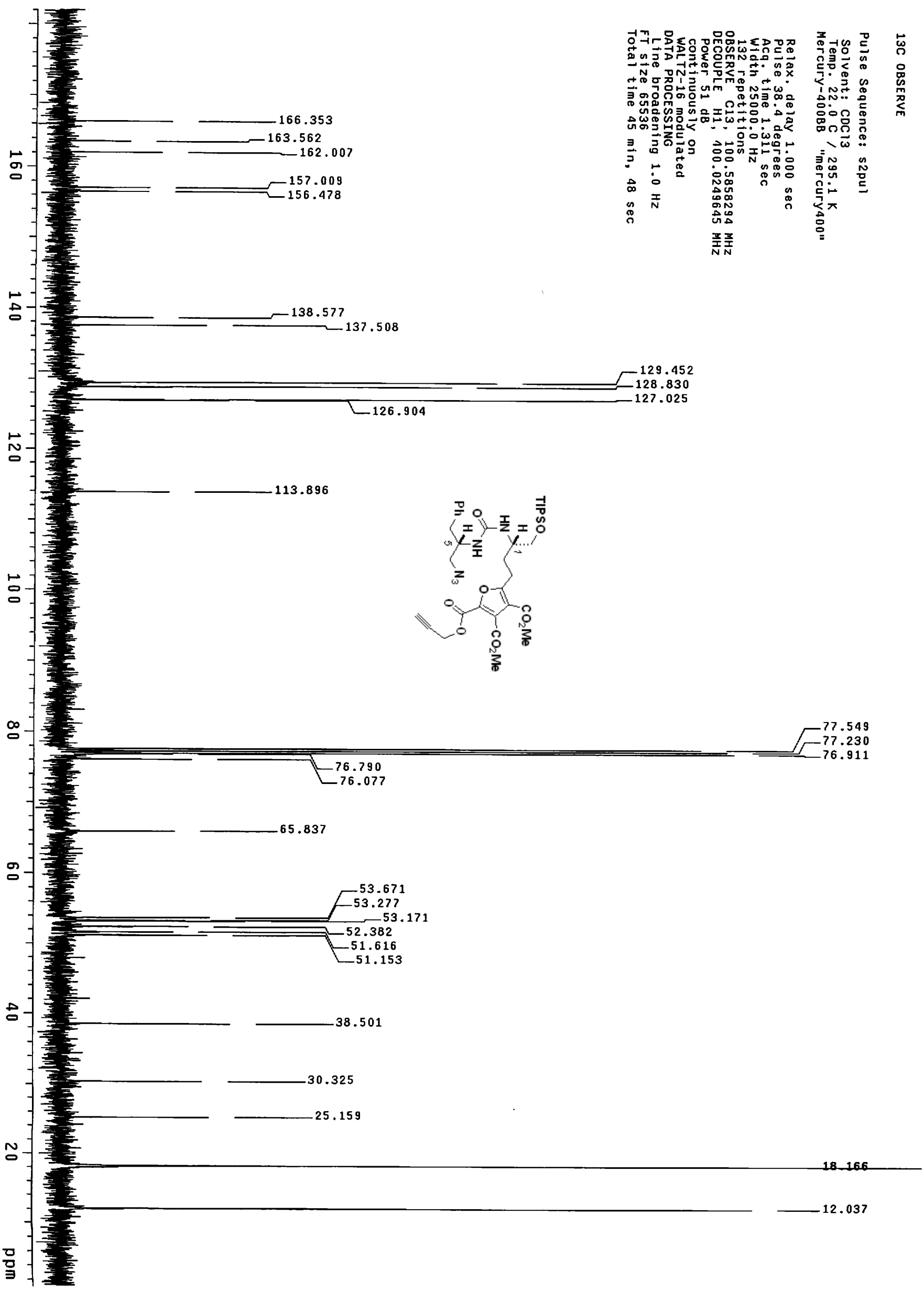


Pulse Sequence: s2pur

Solvent: $\operatorname{CDC} 13$

Temp. $22.0 \mathrm{C} / 295.1 \mathrm{~K}$

Relax. delay $0.120 \mathrm{sec}$
Pulse 64.3 degrees

Acq. time $2.731 \mathrm{sec}$

Width $5995.2 \mathrm{~Hz}$

16 repetilions 00.0229423

DATA PROCESSING

Line broadening $0.1 \mathrm{~Hz}$

FT size 32768

Total time $1 \mathrm{~min}, 4 \mathrm{sec}$
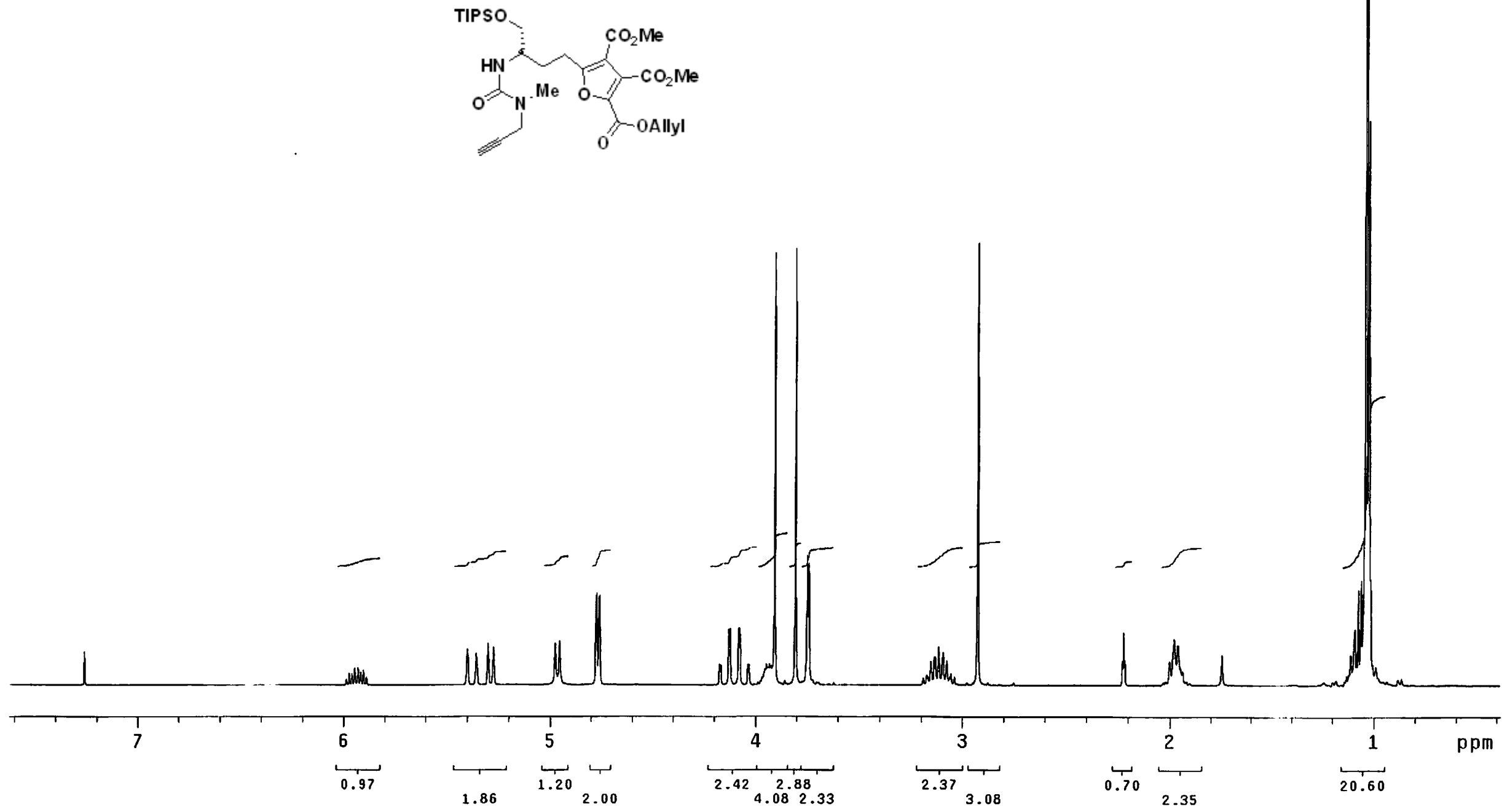


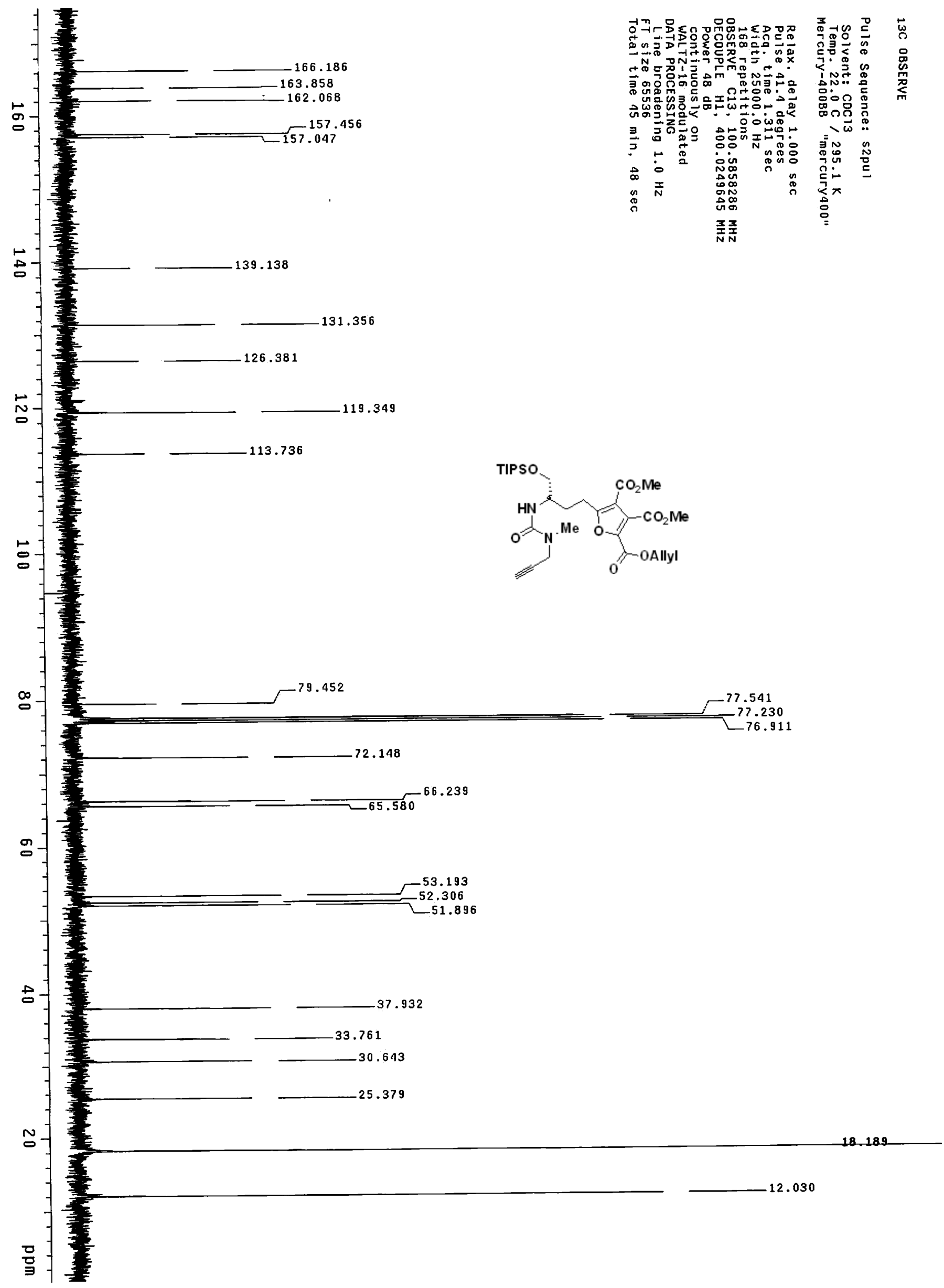




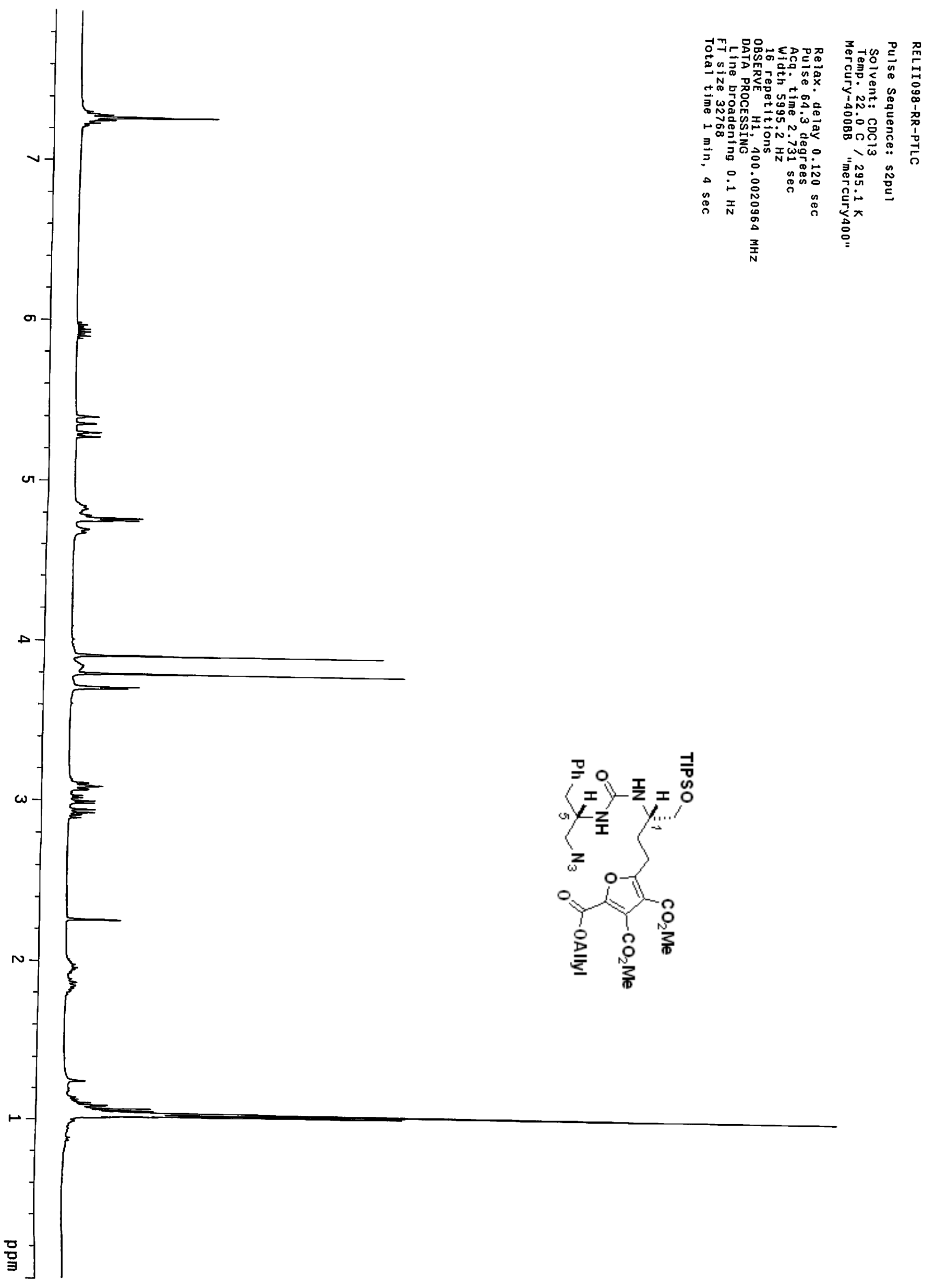


Pulse Sequence: s2pul

Solvent: $\operatorname{CDCl}_{3}$

Temp. $22.0 \mathrm{C} / 295$, $\mathrm{K}$

Pulse 38.4 degrees

Acq time $1.311 \mathrm{sec}$

width $25000.0 \mathrm{~Hz}$

372 repetitions

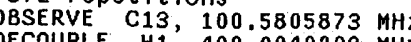

Power 51 dB, 400.0040902 MHZ

cont inuous ly

WALTZ-16 modulated

DATA PROCESSING

Line broadening $1.0 \mathrm{~Hz}$

rotal time $28 \mathrm{~min}, 41 \mathrm{sec}$

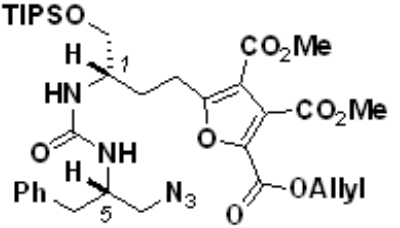


RELII 098-RS-PTLC

Pulse Sequence: s2pul

Solvent: $\mathrm{CDC} 13$

Temp. $22.0 \mathrm{C} / 295.1 \mathrm{~K}$

File: RELII098-RS-PTLC
Mercury-400BB "mercury 400

Relax. delay $0.120 \mathrm{sec}$

Pulse 64.3 degrees

Acq. time $2.731 \mathrm{sec}$

16 repetitions

OBSERVE H1, 400.0020964 MHZ

DATA PROCESSING

Line broadening $0.1 \mathrm{~Hz}$

Total time $1 \mathrm{~min}, 4 \mathrm{sec}$

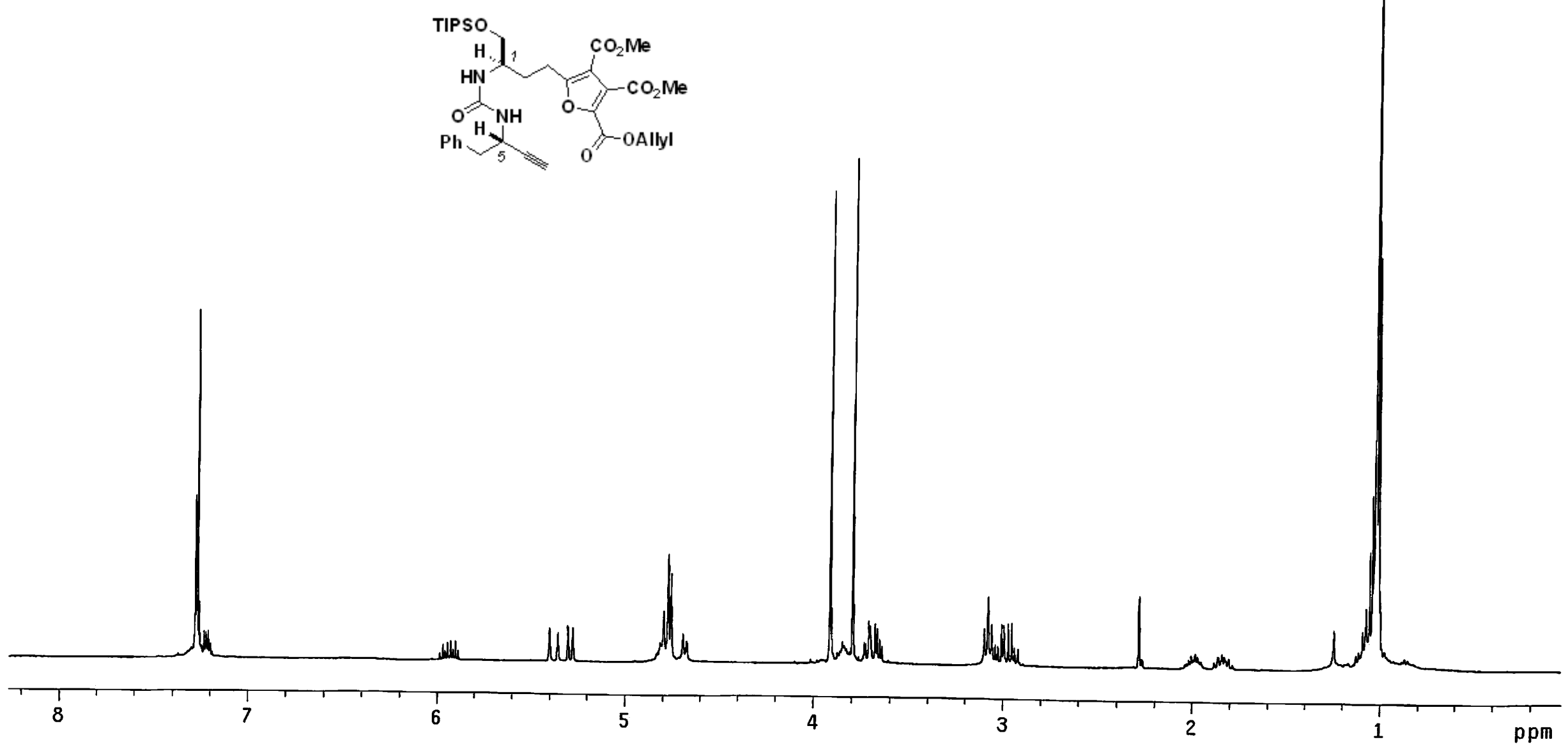

I:

I

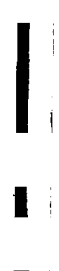


Pulse Sequence: s2pur

Solvent: $\operatorname{CoC} 13$

Temp. 22.0 C / 295 . $\mathrm{K}$

("mercury 400 "

Pulse 38.4 degrees

Acq time $1.311 \mathrm{sec}$

264 repetitions

OBSERVE C13, 100.5805873 MHZ

POWP $51 \mathrm{HB}, 400.0040902 \mathrm{MHZ}$

cont inuous ly

WALTZ-16 modulated

DATA PROCESSING

Line broadening $1.0 \mathrm{~Hz}$

Total time $28 \mathrm{~min}, 44 \mathrm{sec}$

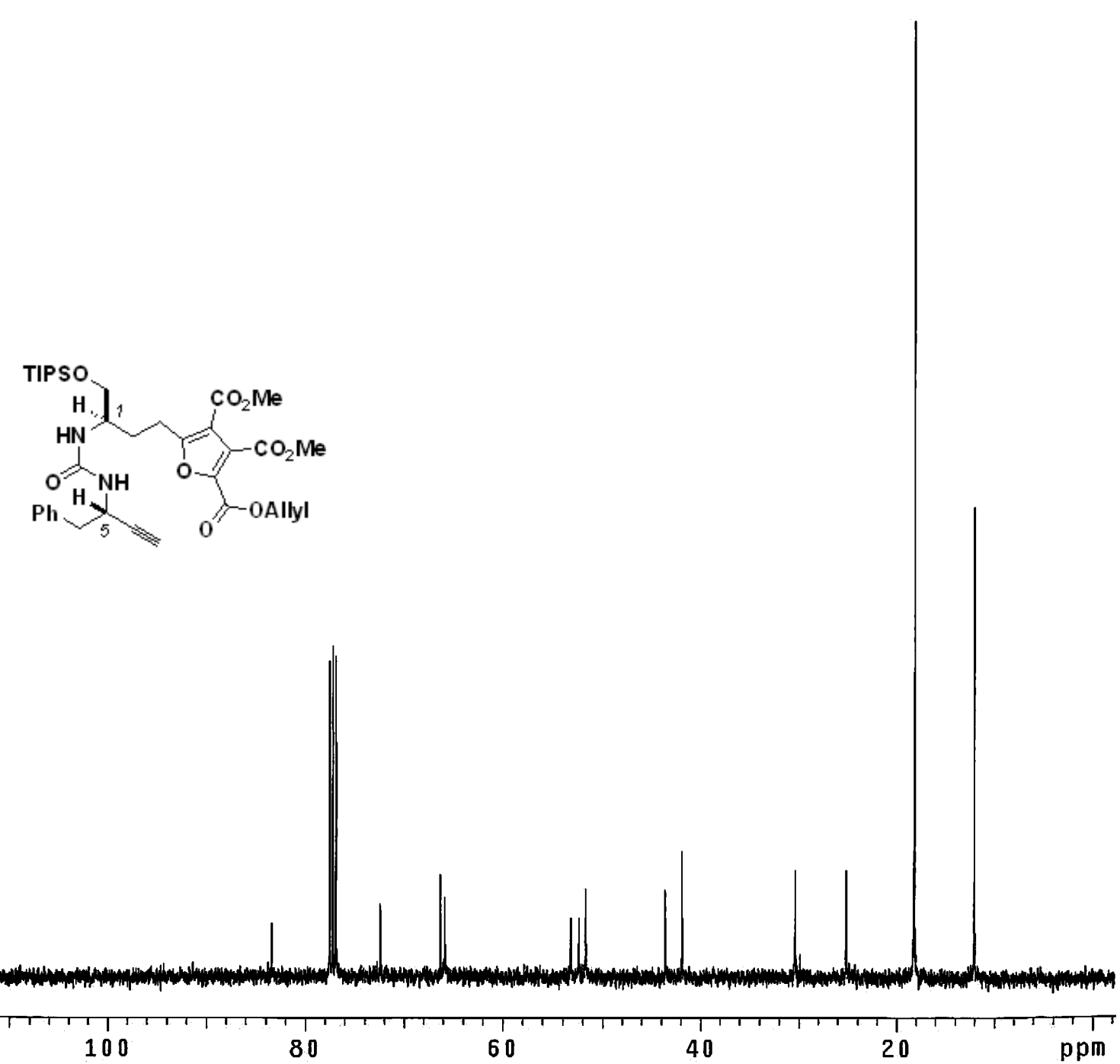


RELII 098 -RS-AMIDE

Pulse Sequence: s2pul

Solvent: $\operatorname{CoC} 13$

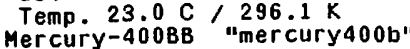

Pulse 77.1 degrees

Acq. time $2.731 \mathrm{se}$

Width $5998.8 \mathrm{~Hz}$

16 repetitions

OBSERVE PROCESSING

Line broadening $0.1 \mathrm{~Hz}$

FT size 32768
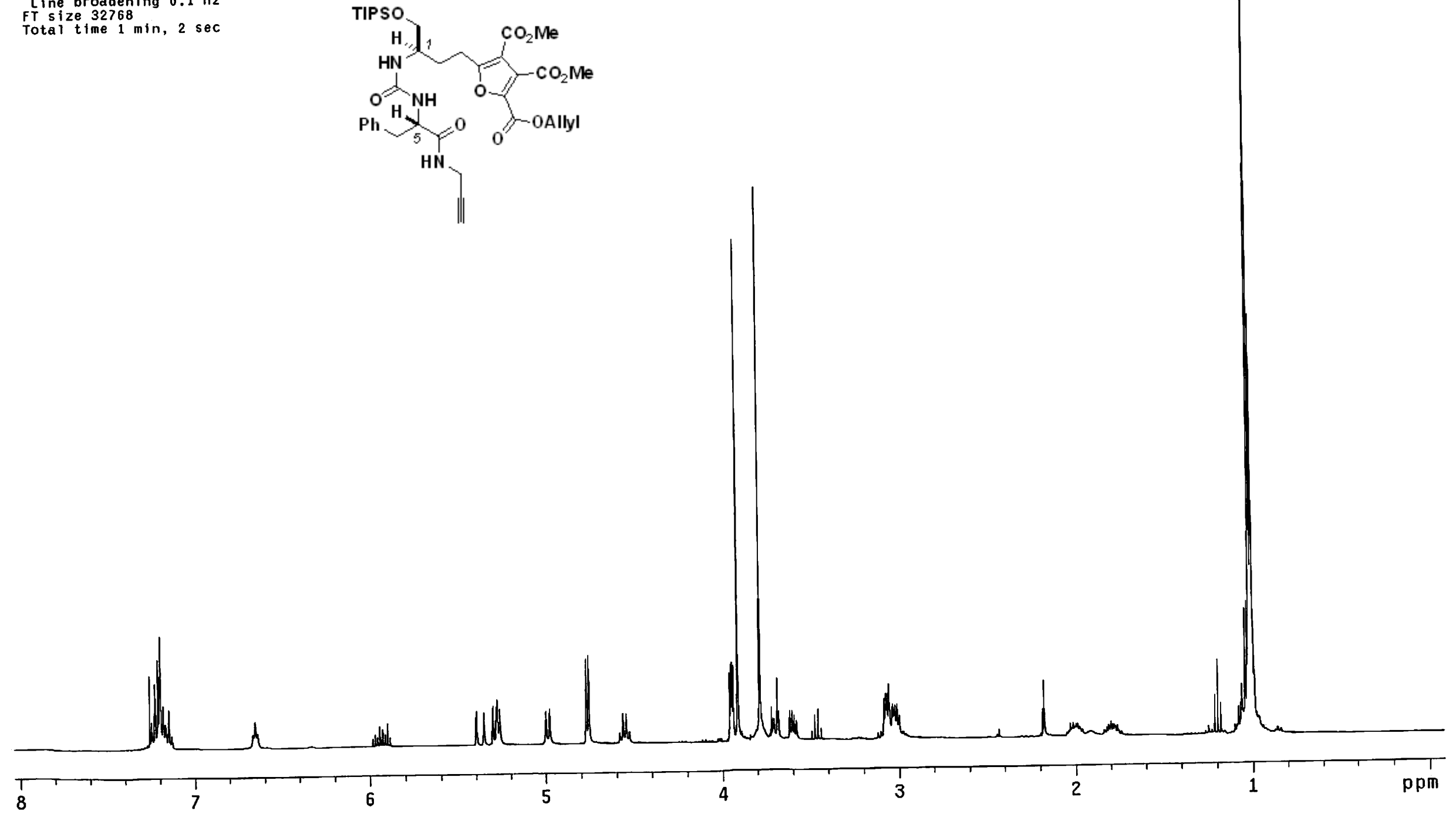
Pulse Sequence: s2pul

Solvent: $\operatorname{CDC}_{13}$

Temp. $23.0 \mathrm{C} / 296.1 \mathrm{~K}$
Mercury-400B

Pulse 36.7 degrees

Acq. time $1.311 \mathrm{sec}$

Width $23980.8 \mathrm{~Hz}$

OBSERVE C13, 100.5389801 MHZ

DECOUPLE H1, 100.5389801 MH

Power $47 \mathrm{~dB}$

cont inuous ly on

WALTZ-16 modulate

WATA PROCESSING

FT ne broadening $1.0 \mathrm{~Hz}$

Total time $28 \mathrm{~min}, 42 \mathrm{sec}$

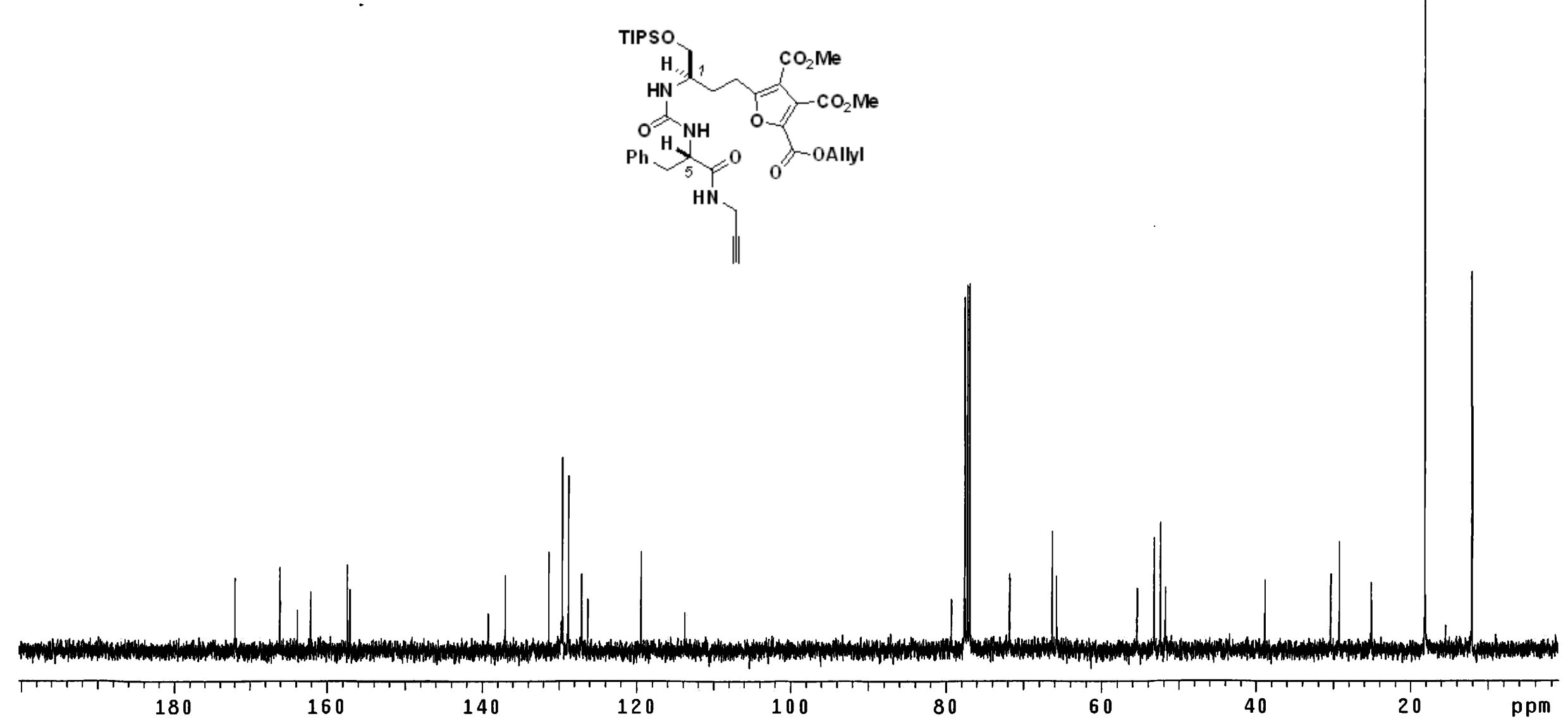




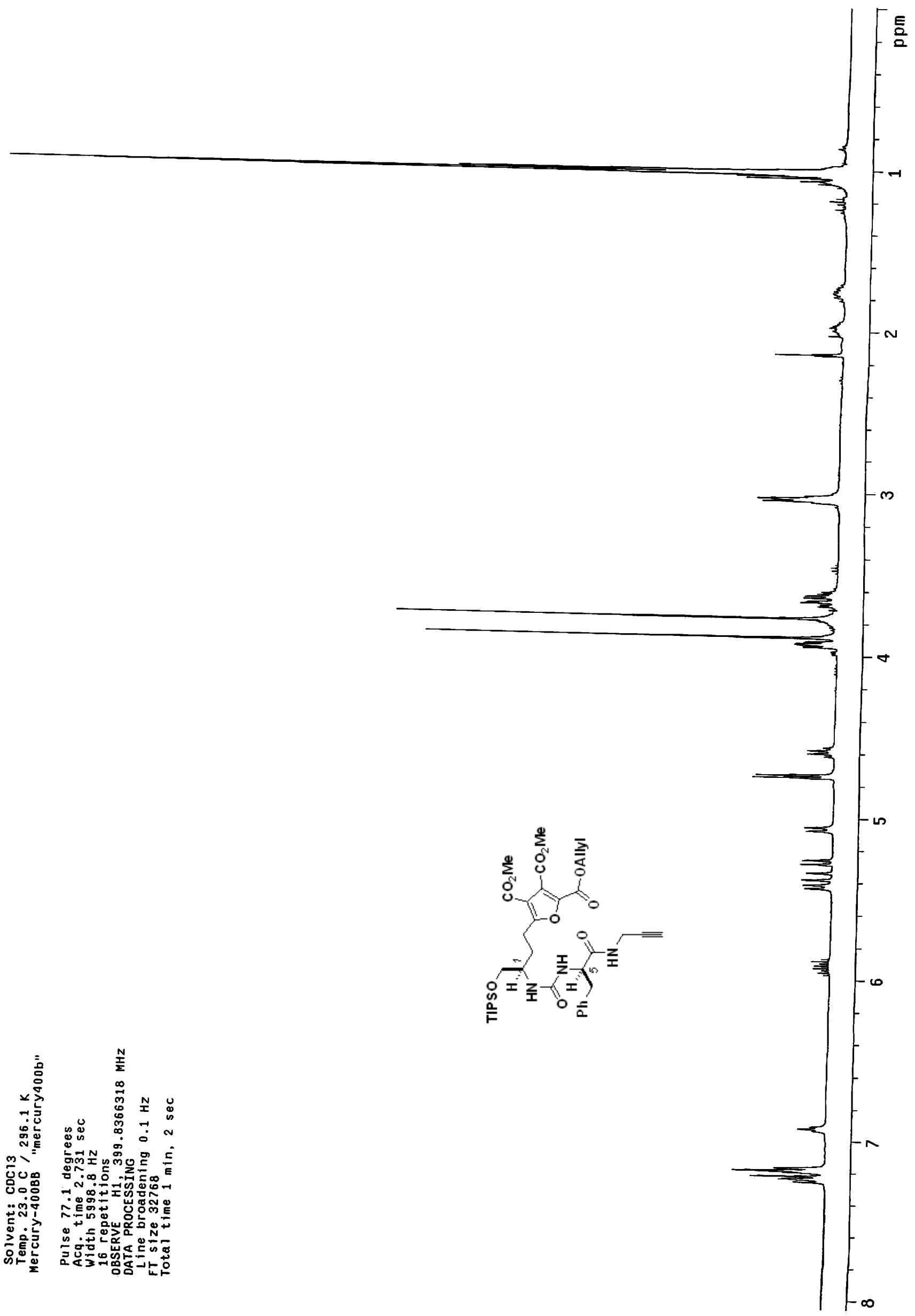


RELII 098-RR-AMIDE-C13

Pulse Sequence: s2pu

Solvent: $\mathrm{CDC}_{13}$

Temp. $23.0 \mathrm{C} / 296.1 \mathrm{~K}$
Mercury-400B

Pulse 36.7 degrees

Acq $\mathrm{time} 1.311 \mathrm{sec}$

idth 23980.8 H

OBSERVE C13, $100.5389816 \mathrm{MHZ}$

DECOUPLE H1, 399.8385865 MHZ

Power A7 dB

COA tinuous iy on

DATA PROCESSING

Line broadening $1.0 \mathrm{~Hz}$

FT size 65536
Total time $28 \mathrm{~min}, 12 \mathrm{sec}$
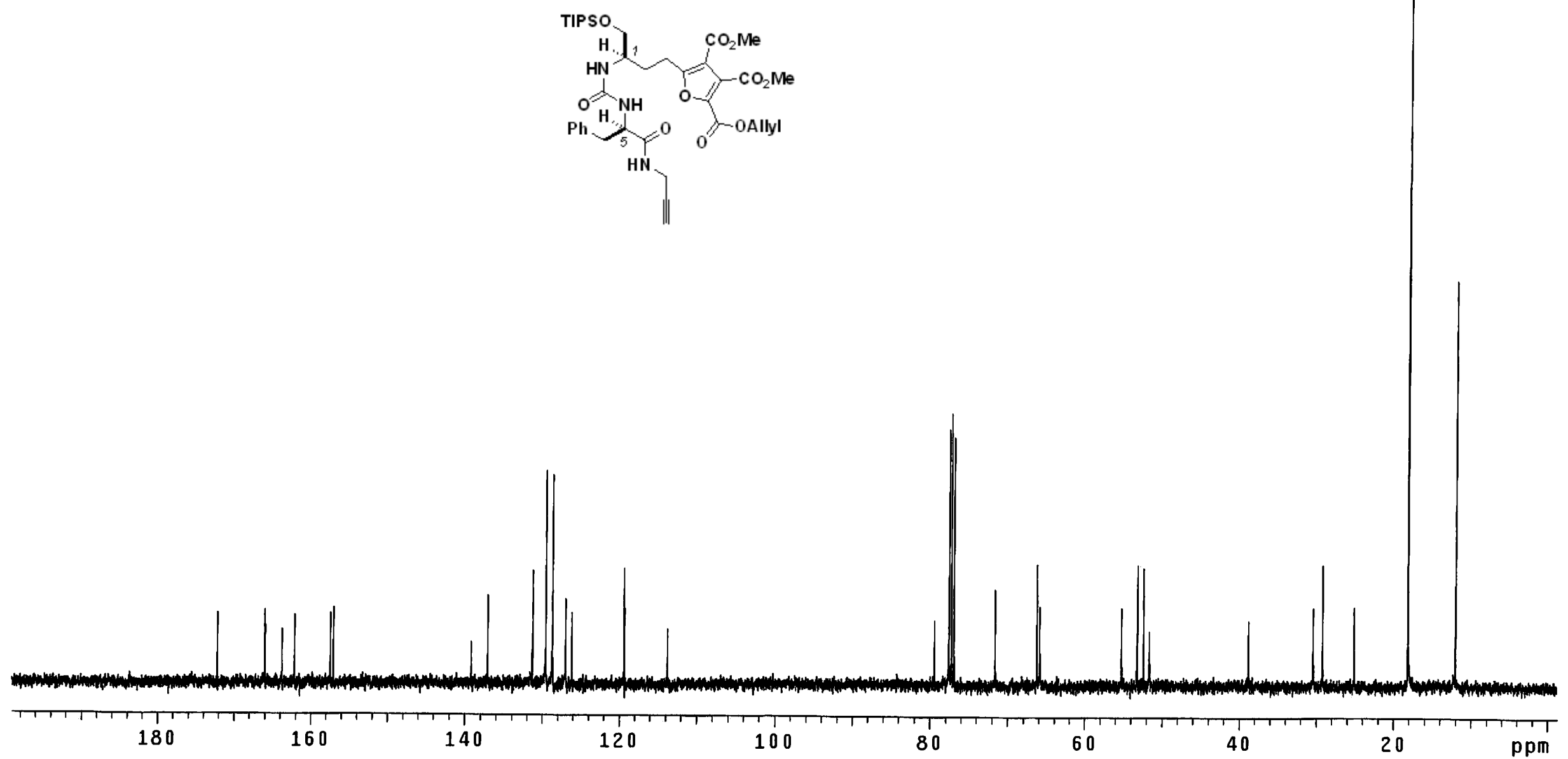

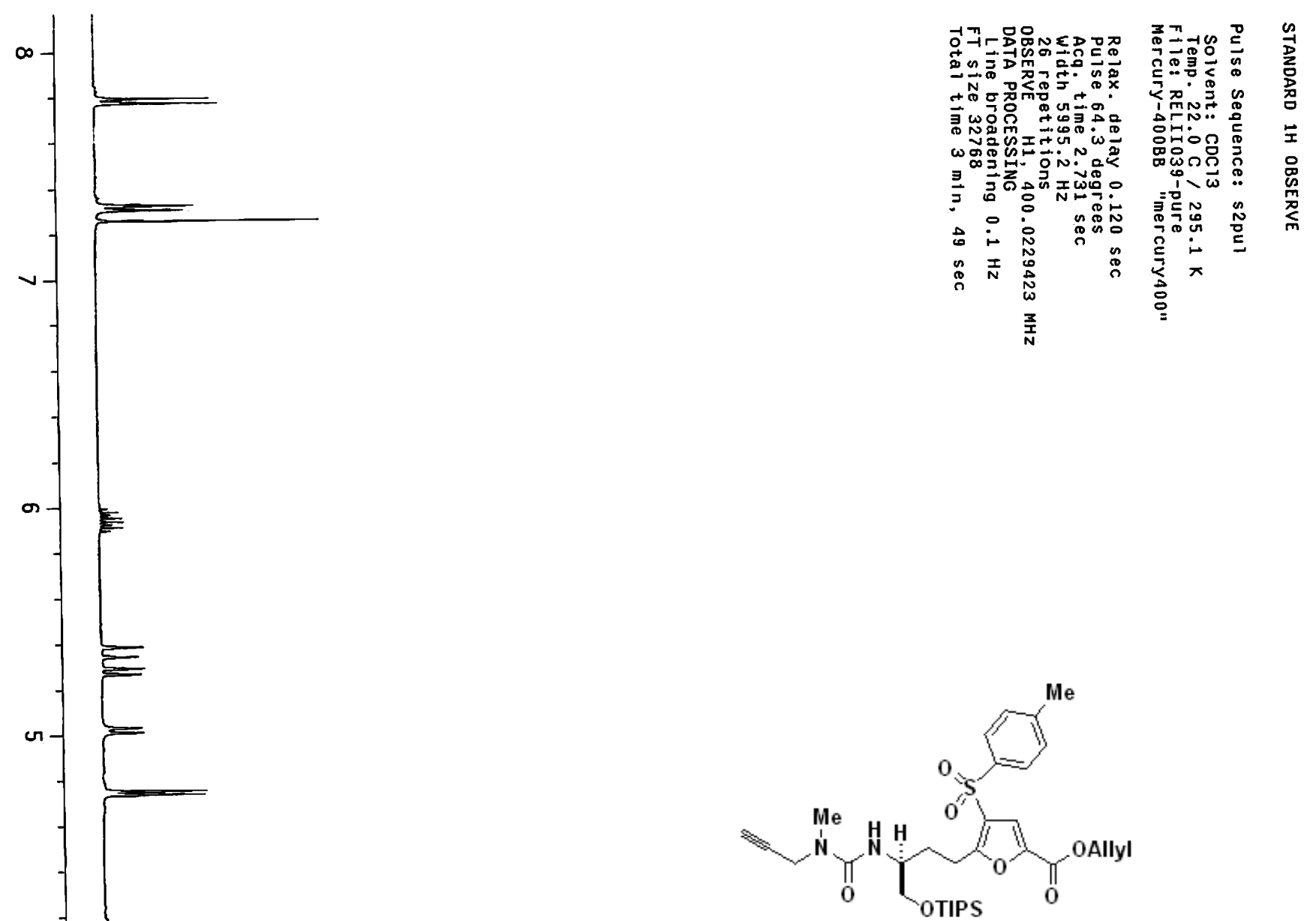


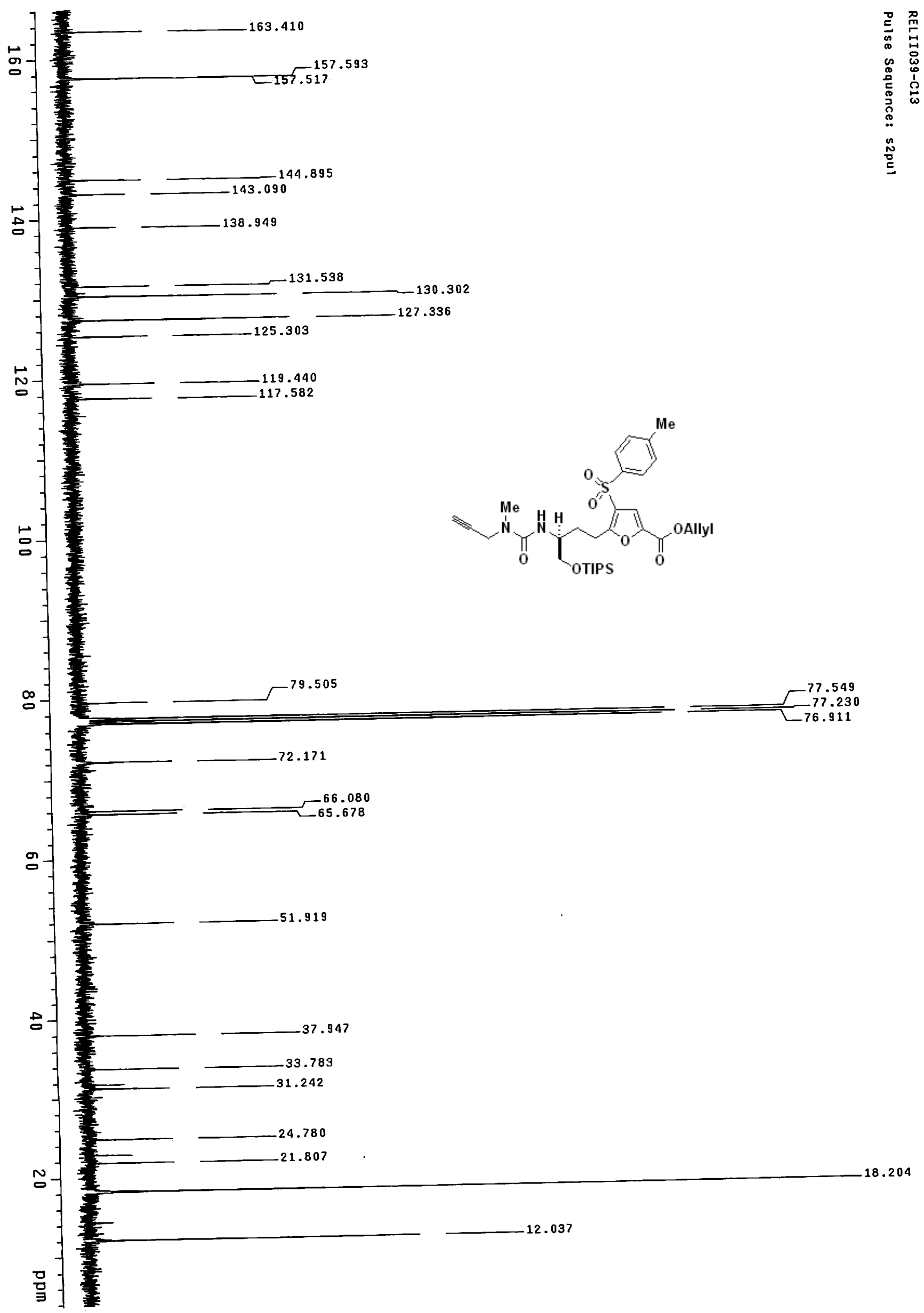


RELII031-roxazole

Pulse Sequence: s2pul

Solvent: $\operatorname{CDC}_{13}$

Temp. $22.0 \mathrm{C} / 295.1 \mathrm{~K}$

File: RELII 031-1

Re lax. de lay $0.120 \mathrm{sec}$

Acq. time $2.731 \mathrm{sec}$

W1dth $5995.2 \mathrm{~Hz}$

16 repetitions $0229423 \mathrm{MHZ}$

OBSERV H1, $400.0229423 \mathrm{MHZ}$

Line broadening $0.1 \mathrm{~Hz}$

FT size 32768
Total time $1 \mathrm{~min}, 4 \mathrm{sec}$

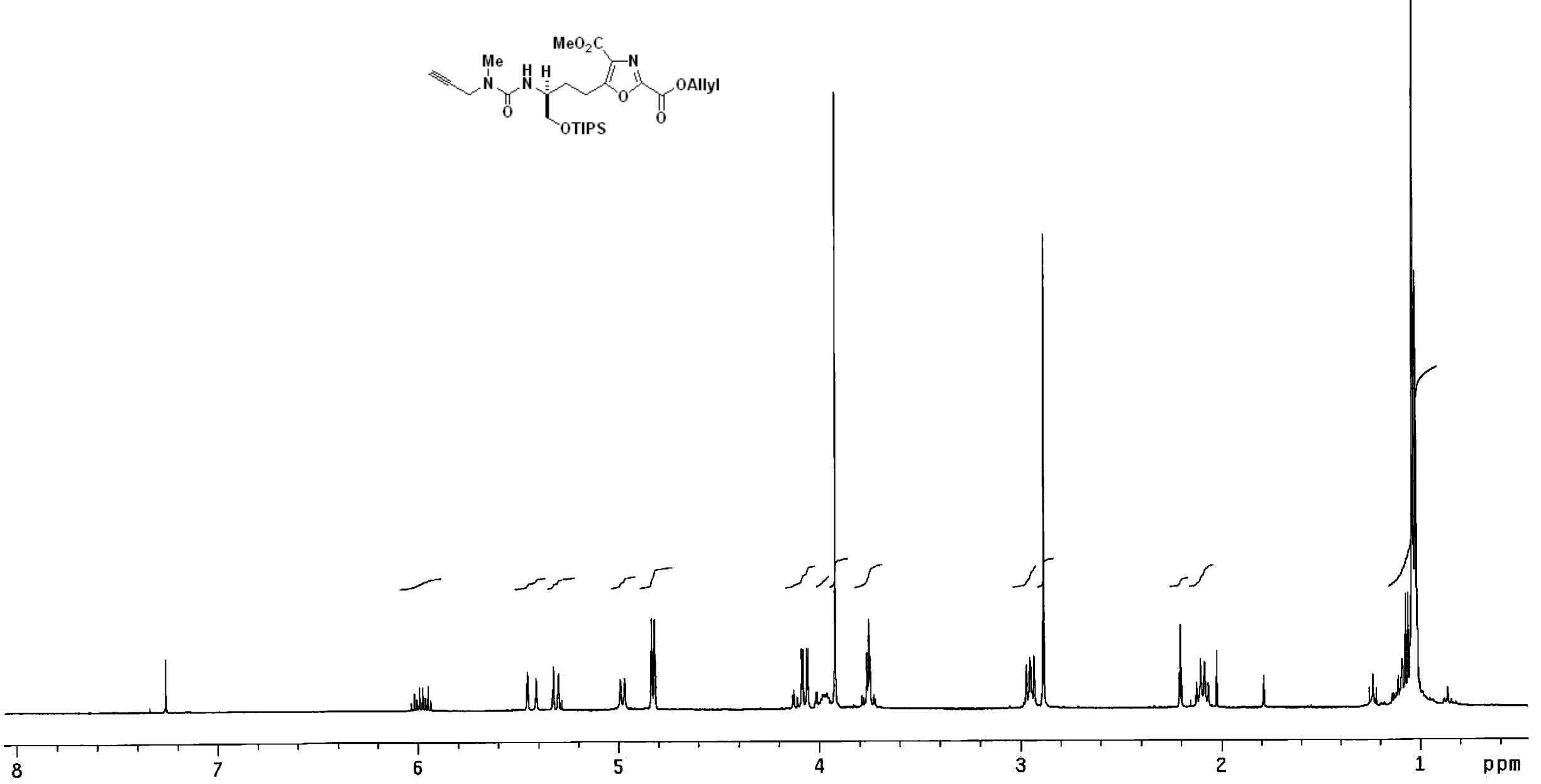


13C OBSERVE

Pulse Sequence: s2pul

Solvent: $\operatorname{CDC} 13$

Temp. $22.0 \mathrm{C} / 295.1 \mathrm{~K}$
Mercury-400B

Relax. delay $1.000 \mathrm{sec}$
Pulse 38.4 degrees

Acq. time $1.311 \mathrm{sec}$

Width $25000.0 \mathrm{~Hz}$

132 repetitions

DECOUPLE HI, $400.5858286 \mathrm{MHZ}$

cont inuous ly on

WALTZ-16 modulate

DATA PROCESSING

Line broadening $1.0 \mathrm{~Hz}$

Total time $45 \mathrm{~min}, 48 \mathrm{sec}$
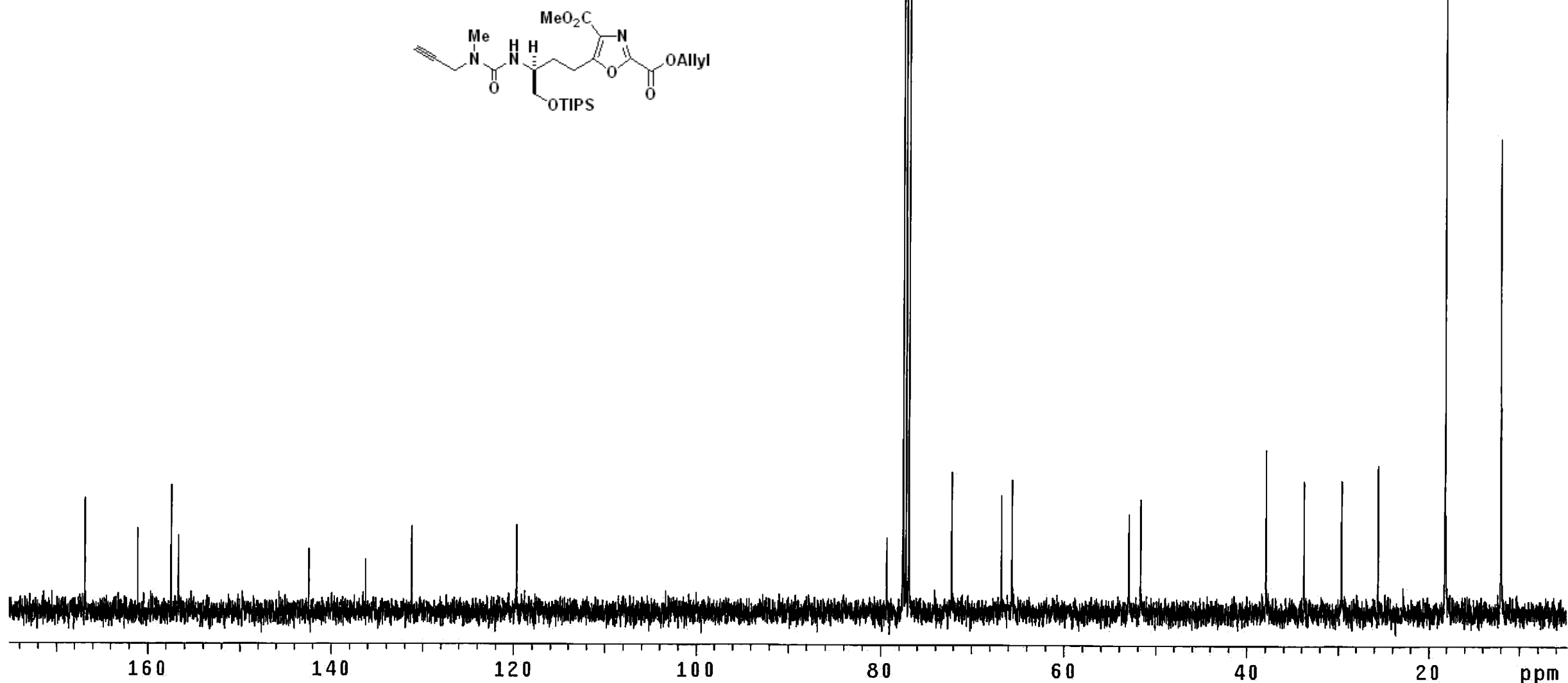
STANDARD PROTON PARAMETERS

Pulse Sequence: s2pul

Solvent: DMSO

Temp. $23.0 \mathrm{C} / 296.1 \mathrm{~K}$

Relax, delay $0.200 \mathrm{sec}$

Acq. time 2.049 sec

Width $7996.0 \mathrm{~Hz}$

60 repetitions

OBSERVE H1, 499.7502831 MHZ

DATA PROCESSING

LT ne broadening $0.1 \mathrm{~Hz}$

Total time $9 \mathrm{~min}, 42 \mathrm{sec}$
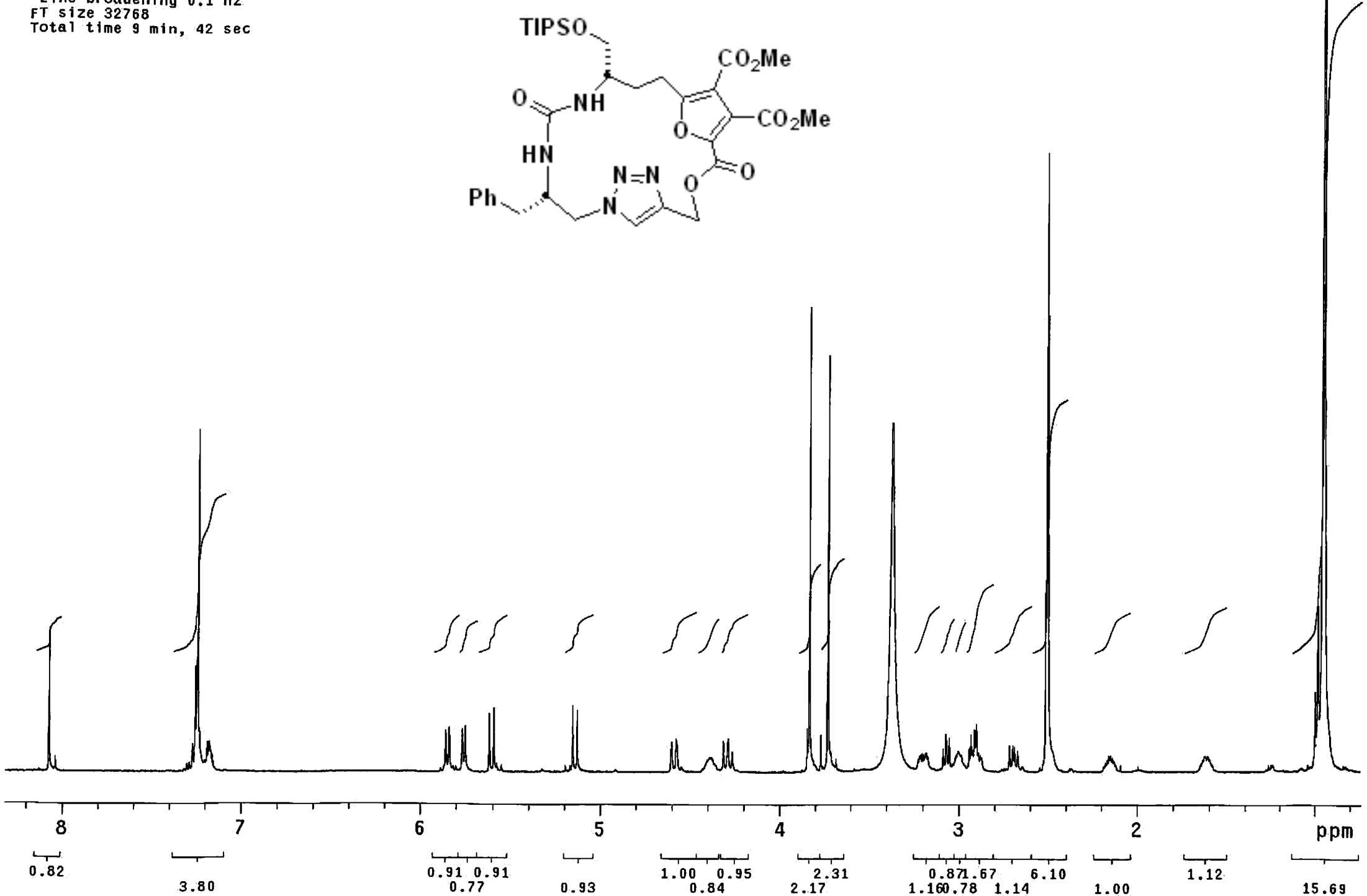
RELI293-C13

Pulse Sequence: s2pul

Solvent: $\mathrm{CDC}_{13}$

$296,1 K$

Mercury-400BB "mercury 400 b"

Relax. delay $1.000 \mathrm{sec}$

Pulse 36.7 degrees

Acq. time $1.311 \mathrm{sec}$

Width $23980.8 \mathrm{~Hz}$

OBSERVE C13, $100.5389801 \mathrm{MHz}$

DECOUPLE H1, 399.8385865 MHZ

Power 47 dB

WALTZ-16

DATA PROCESSING

Line broadening $1.0 \mathrm{~Hz}$

Total time $45 \mathrm{~min}, 48 \mathrm{sec}$
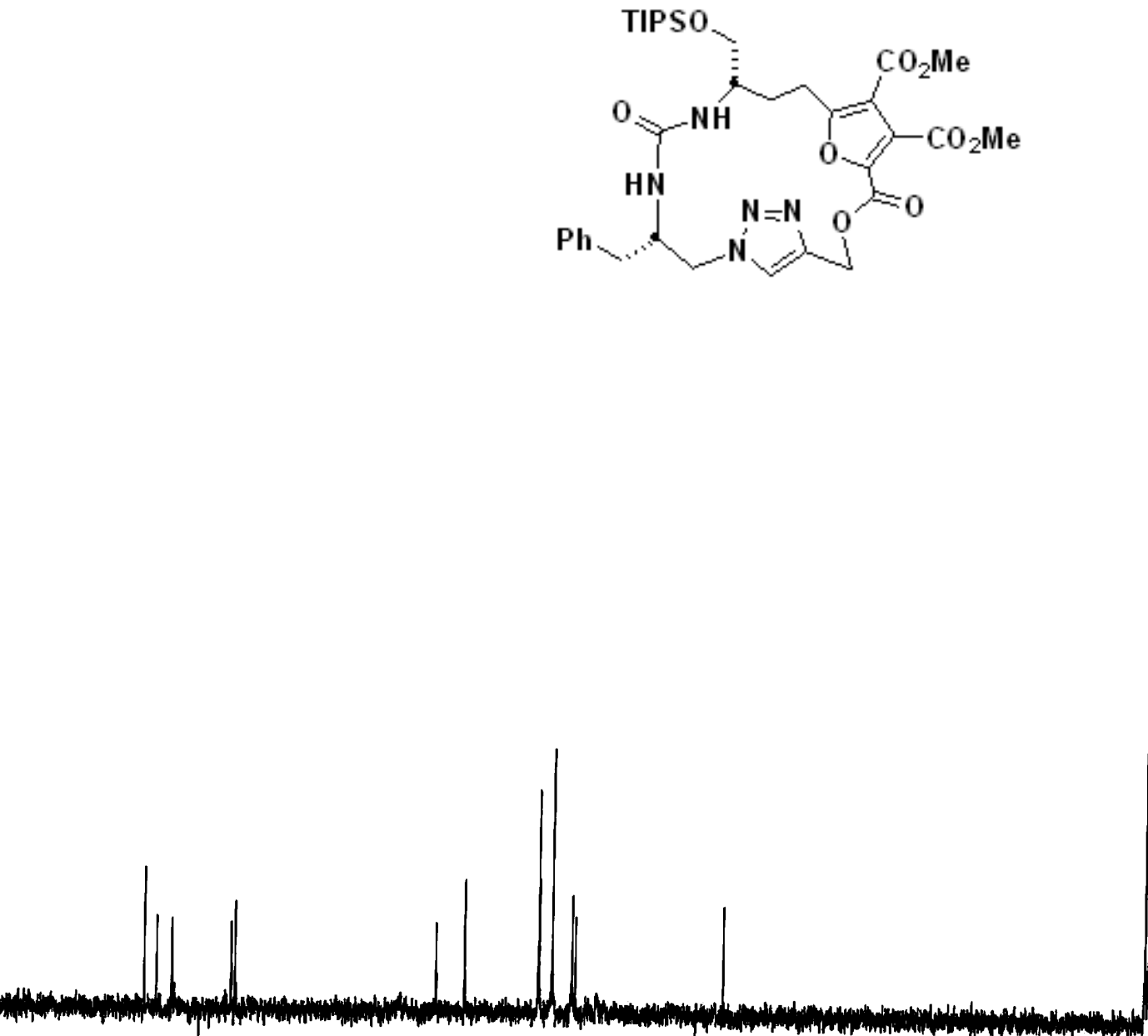


\section{RELI 273-2}

Pulse Sequence: s2pul

Solvent: $\operatorname{CDC} 13$

Mercury-400B $23.00 \mathrm{C} / 296.1 \mathrm{~K}$

Pulse 77.1 degrees

Acq. time 2.731 se

16 repetitions

OBSERVE H1, $399.8366318 \mathrm{MHZ}$

DATA PROCESSING

Line broadening $0.1 \mathrm{~Hz}$

Total time 1 min, $2 \mathrm{sec}$

TIPSO

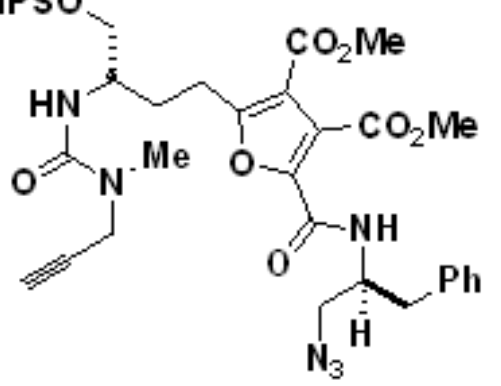

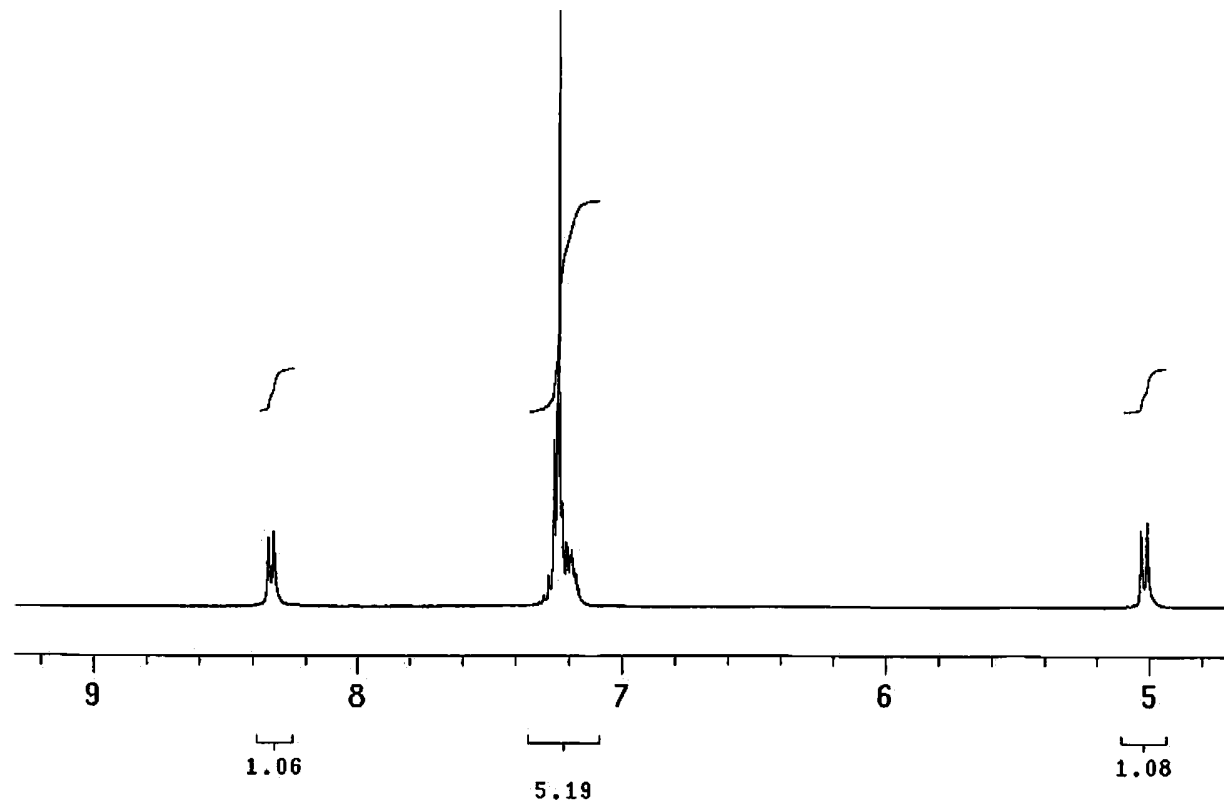

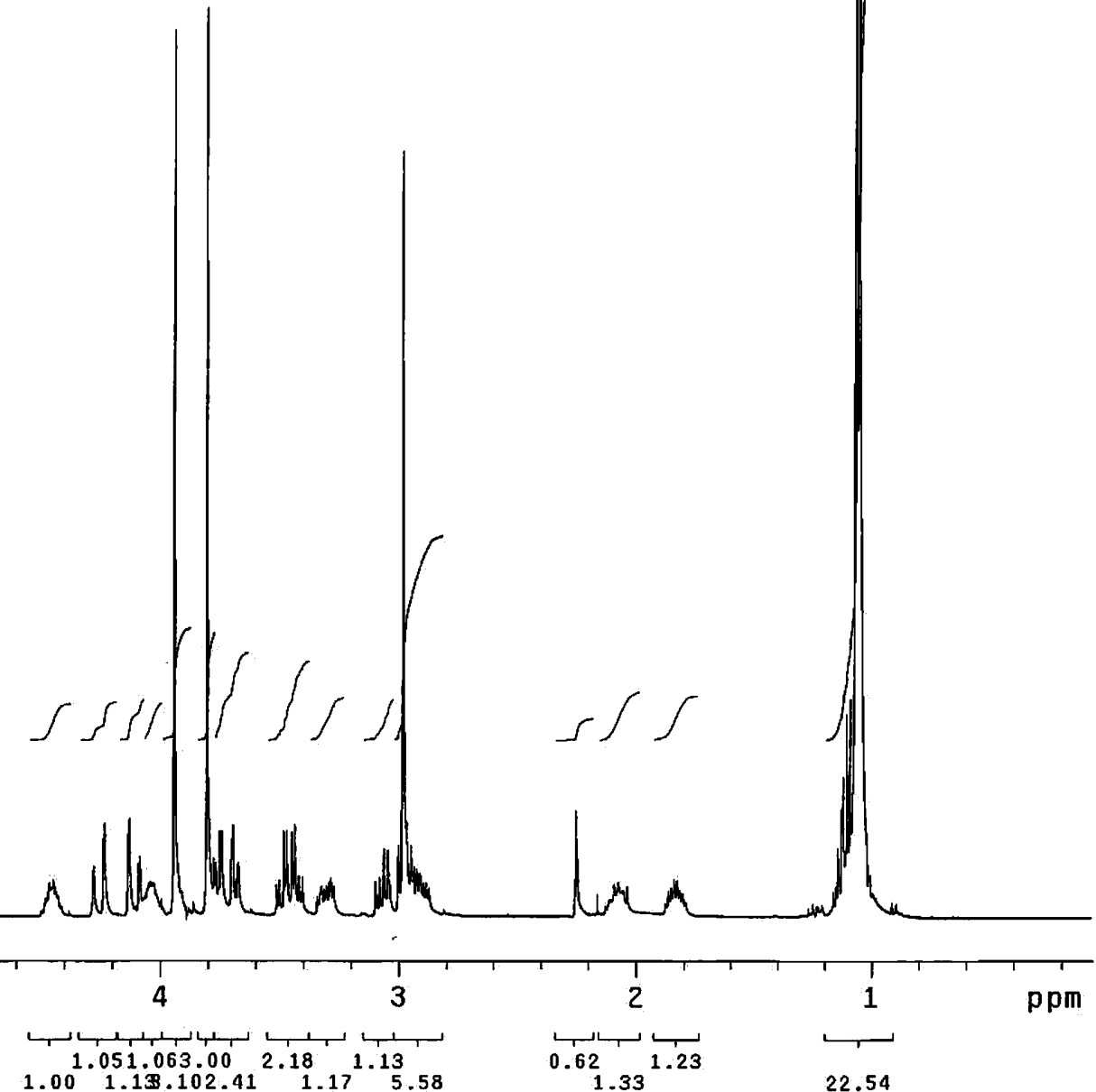




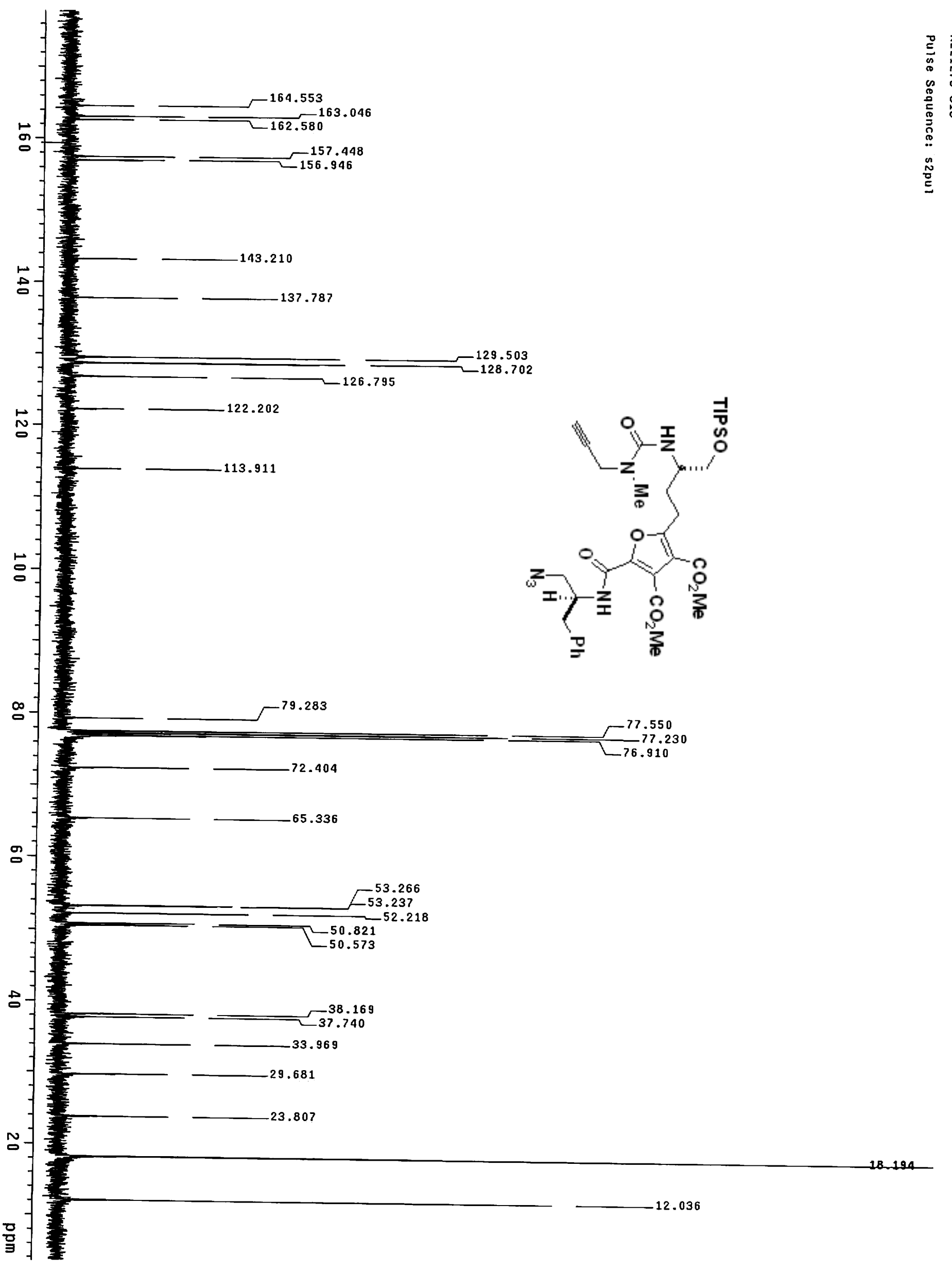


RELII013-1

Purse Sequence: s2pur

Solvent: $\operatorname{CDC} 13$ INOVA-500 "Inova500"

Re lax. de lay $0.200 \mathrm{sec}$

Pulse 65.1 degrees

Acq time 2.049 sec

Width $7996.0 \mathrm{HZ}$

59 repetitions

DATA PROCESSING

Line broadening $0.1 \mathrm{~Hz}$

FT size 32768
Total time $4 \mathrm{~min}, 53 \mathrm{sec}$
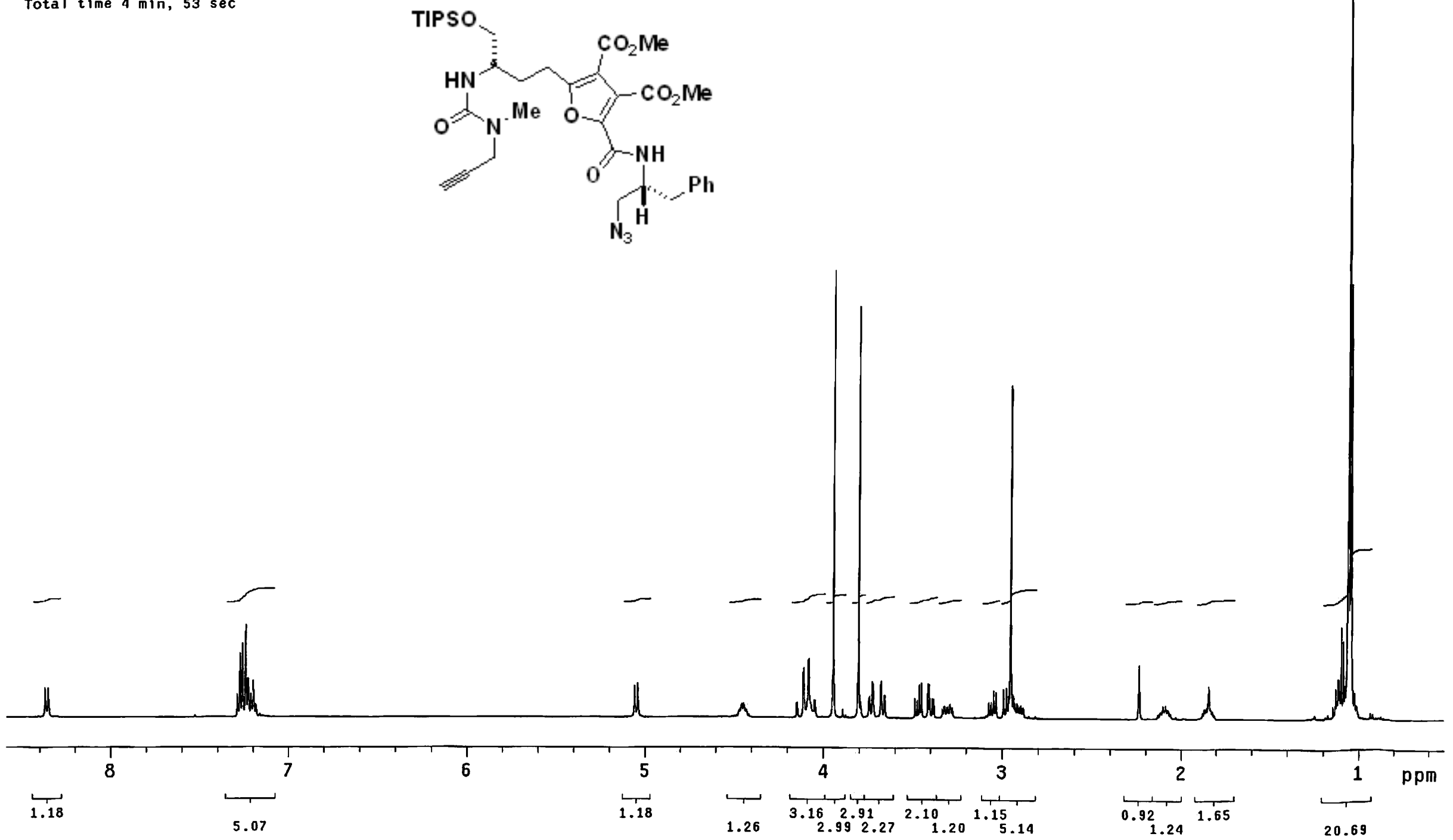
RELII013-C13

Pulse Sequence: s2pur

Solvent: $\operatorname{CoC} 13$

Temp. $23.0 \mathrm{C} / 296.1 \mathrm{~K}$

INOVA-500 "i nova500"

Relax. de lay $1.000 \mathrm{sec}$

Acq. $t$ ime 1.092 sec

Width $29996.3 \mathrm{~Hz}$

420 repetitions

OBSERVE C13, $125.6616946 \mathrm{MHz}$

DECOUPLE H1, 499.7504192 MHz

cont inuous ly on

WALTZ-16 modulate

DATA PROCESSING

Line broadening $1.0 \mathrm{~Hz}$

Total time $35 \mathrm{~min}, 1 \mathrm{sec}$
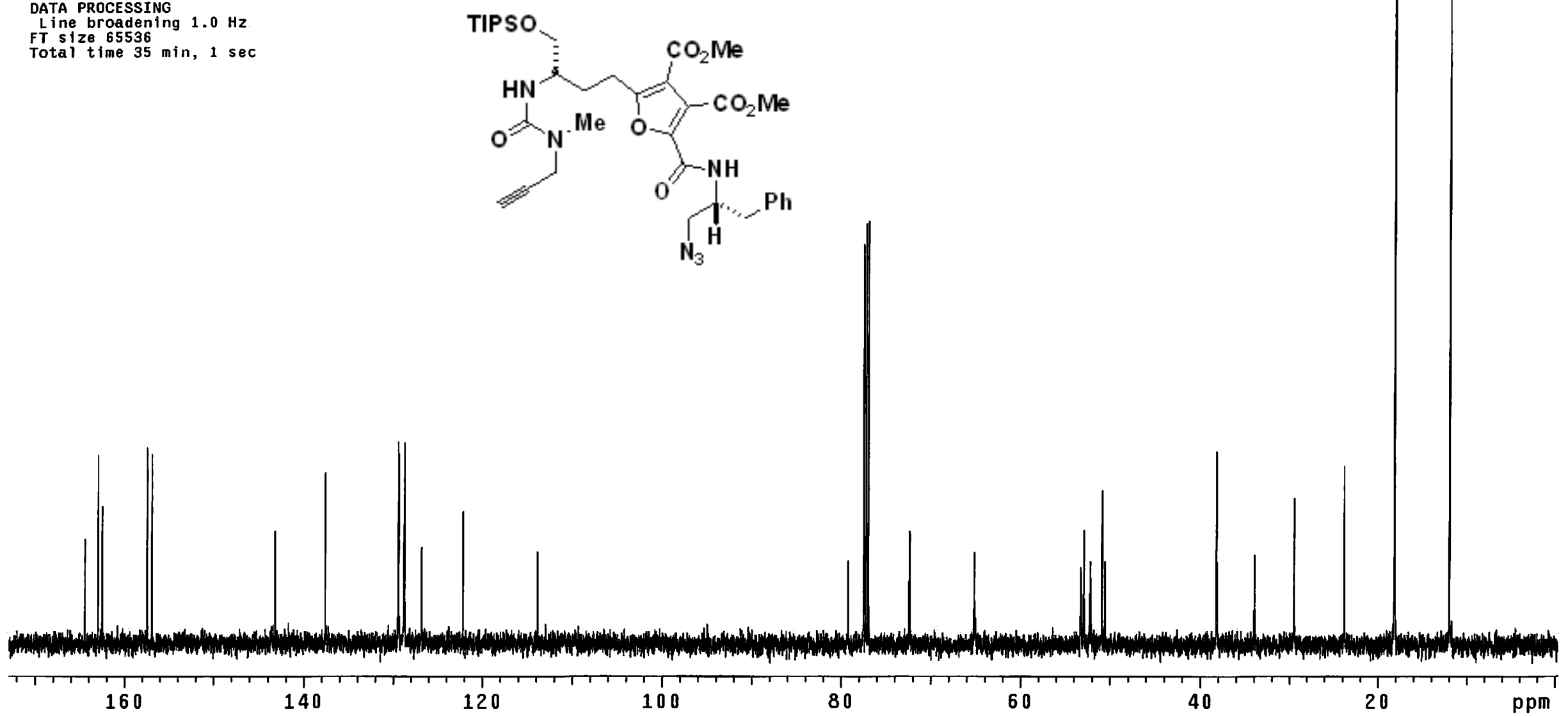
RELI283-1

Pulse Sequence: s2pul

Solvent: $\mathrm{CDCl}_{13}$

emp. $22.0 \mathrm{C} / 295.1 \mathrm{~K}$

Mercury-400BB "mercury400"

Relax. de lay $0.120 \mathrm{sec}$

Pulse 64.3 degrees

Acq. time $2.731 \mathrm{se}$

Width $5995.2 \mathrm{~Hz}$

52 repet tions $0229123 \mathrm{MHZ}$

OBSER H1, $400.0229423 \mathrm{MHZ}$

Line broadening $0.1 \mathrm{~Hz}$

FT size 32768
Total time $7 \mathrm{~min}, 28 \mathrm{sec}$
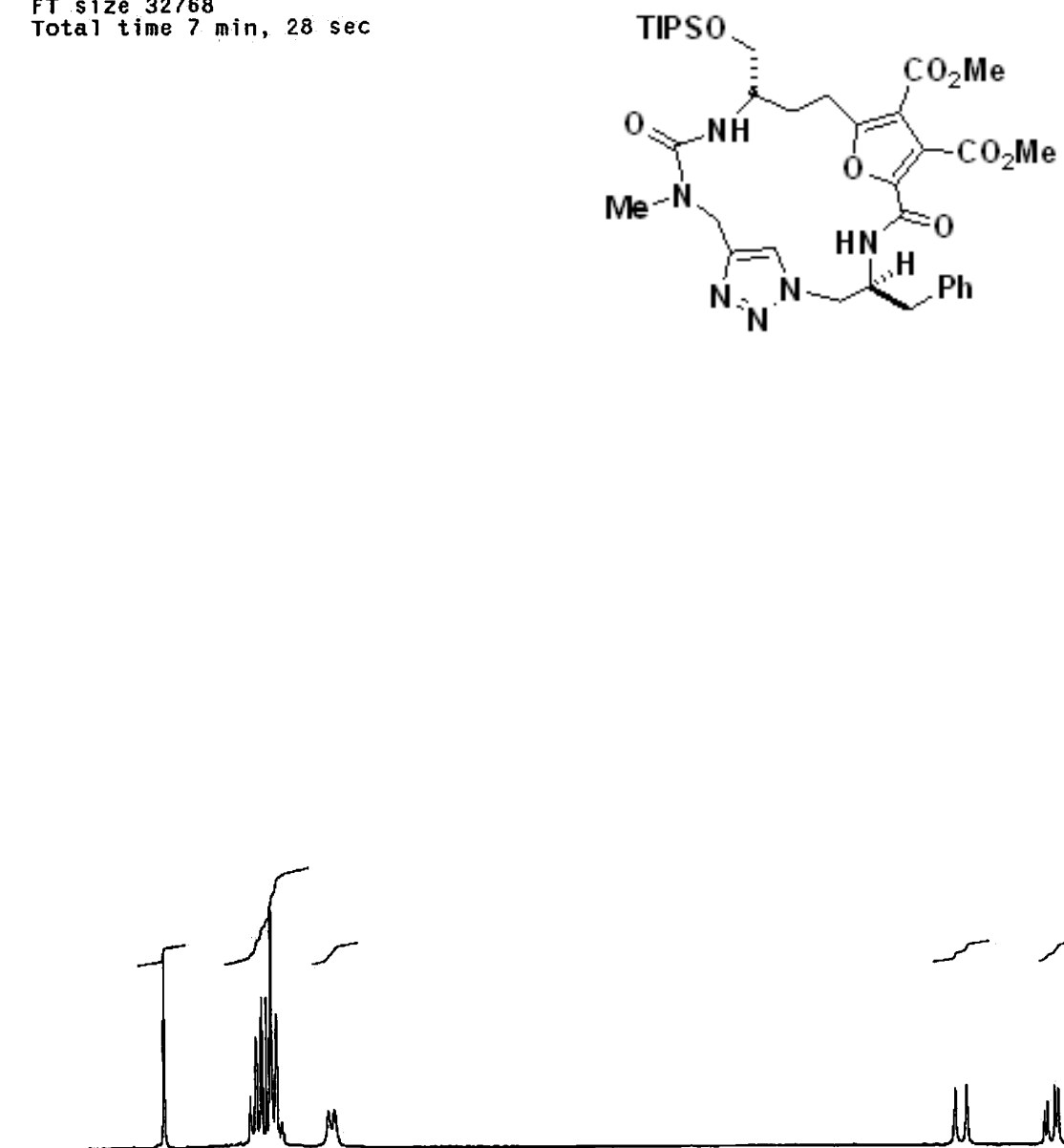

rester
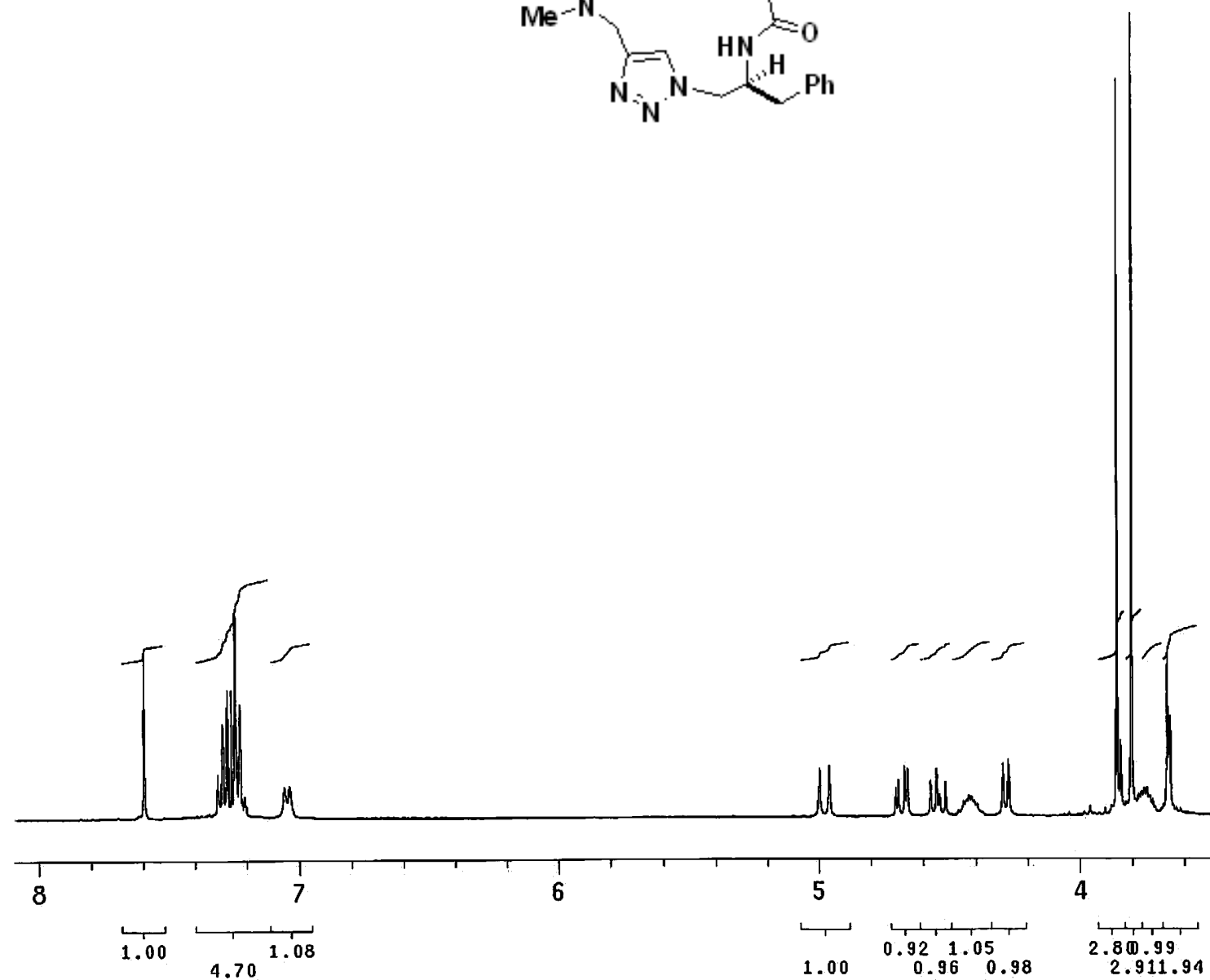

6
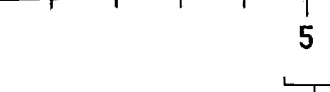

1

0.921 .05

4

2.80 .99

$\begin{array}{ll}8.80 .99 & 2.08 \\ 2.911 .94 & 1.94\end{array}$

$1.00 \quad 0.96 \quad 0.98$ 


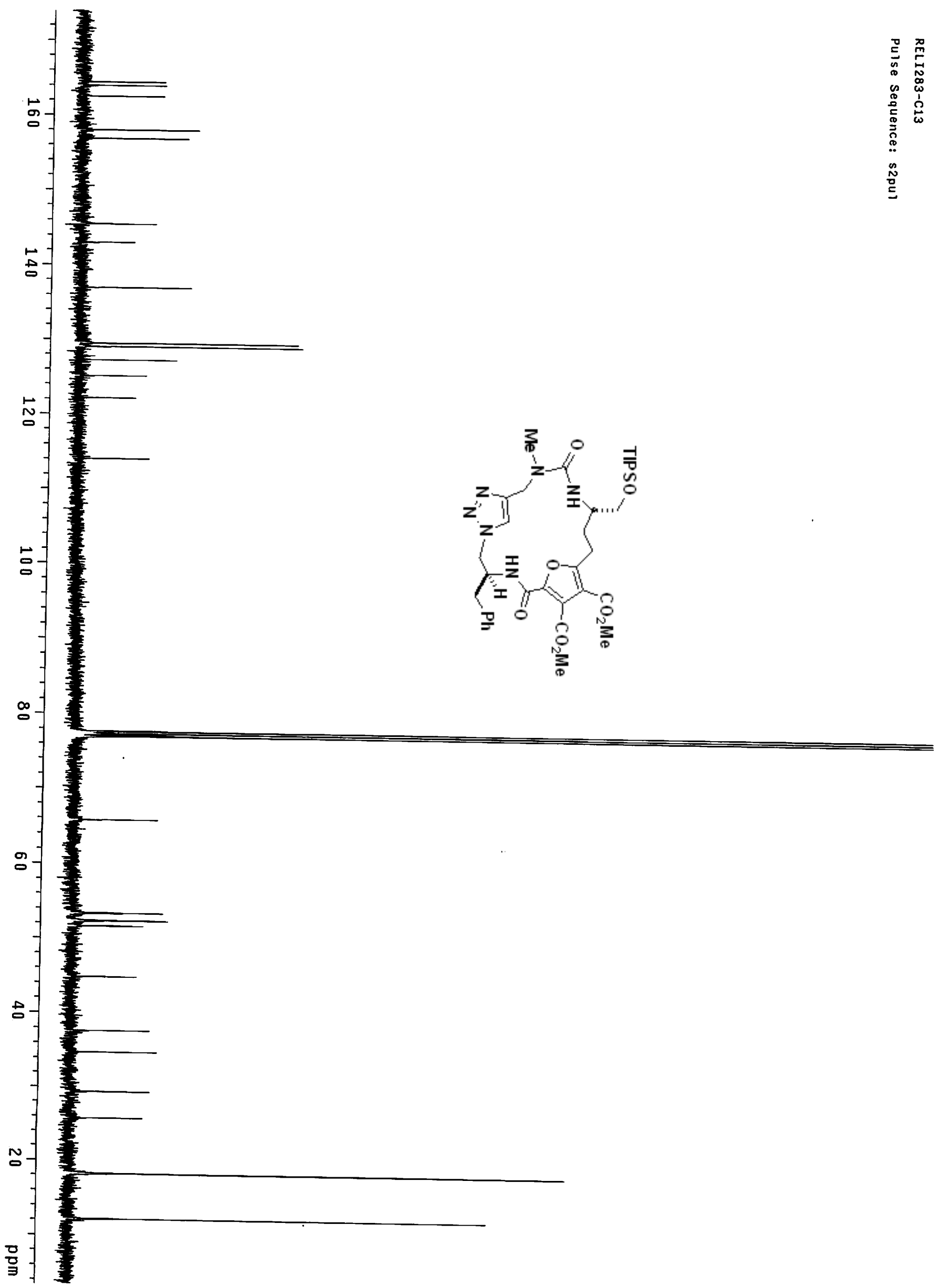


Re lax. de lay $0.200 \mathrm{sec}$

Aca. time $2.049 \mathrm{se}$

Width $7996.0 \mathrm{~Hz}$

OBSERVE tions

OBSERVE H1 $499.7479203 \mathrm{MHZ}$

Line broadening $0.1 \mathrm{~Hz}$

FT size 32768

Total time 0 min, $40 \mathrm{sec}$

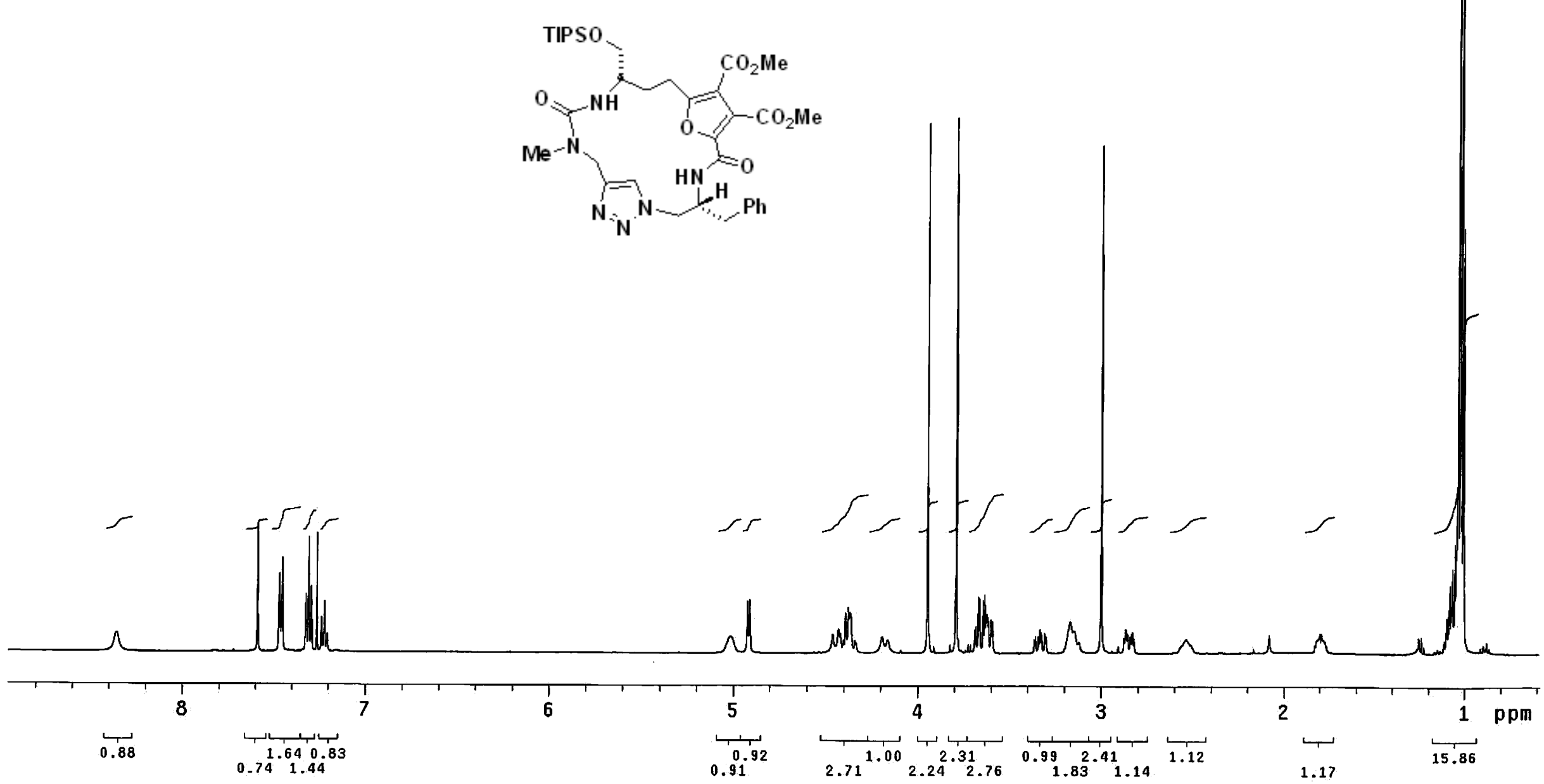


RELII $026-1,2$-therma 1-C13

Pulse Sequence: s2pul

Solvent: $\mathrm{CDCl}_{3}$

Temp. $23.0 \mathrm{C} / 296.1 \mathrm{~K}$

User: $13-14-87$

Relax. de lay $1.000 \mathrm{sec}$

Acq time $1.092 \mathrm{sec}$

Width $29996.3 \mathrm{~Hz}$

OBSERVE C13,125.6616882 MHZ

Power $34 \mathrm{~dB}$ '

cont inuous iy on

WALTZ-16 modulated

DATA PROCESSING

Line broadening $1.0 \mathrm{~Hz}$

Total $\mathrm{t}$ ime $583696 \mathrm{hr}, 59 \mathrm{~m} / \mathrm{n}, 44 \mathrm{sec}$

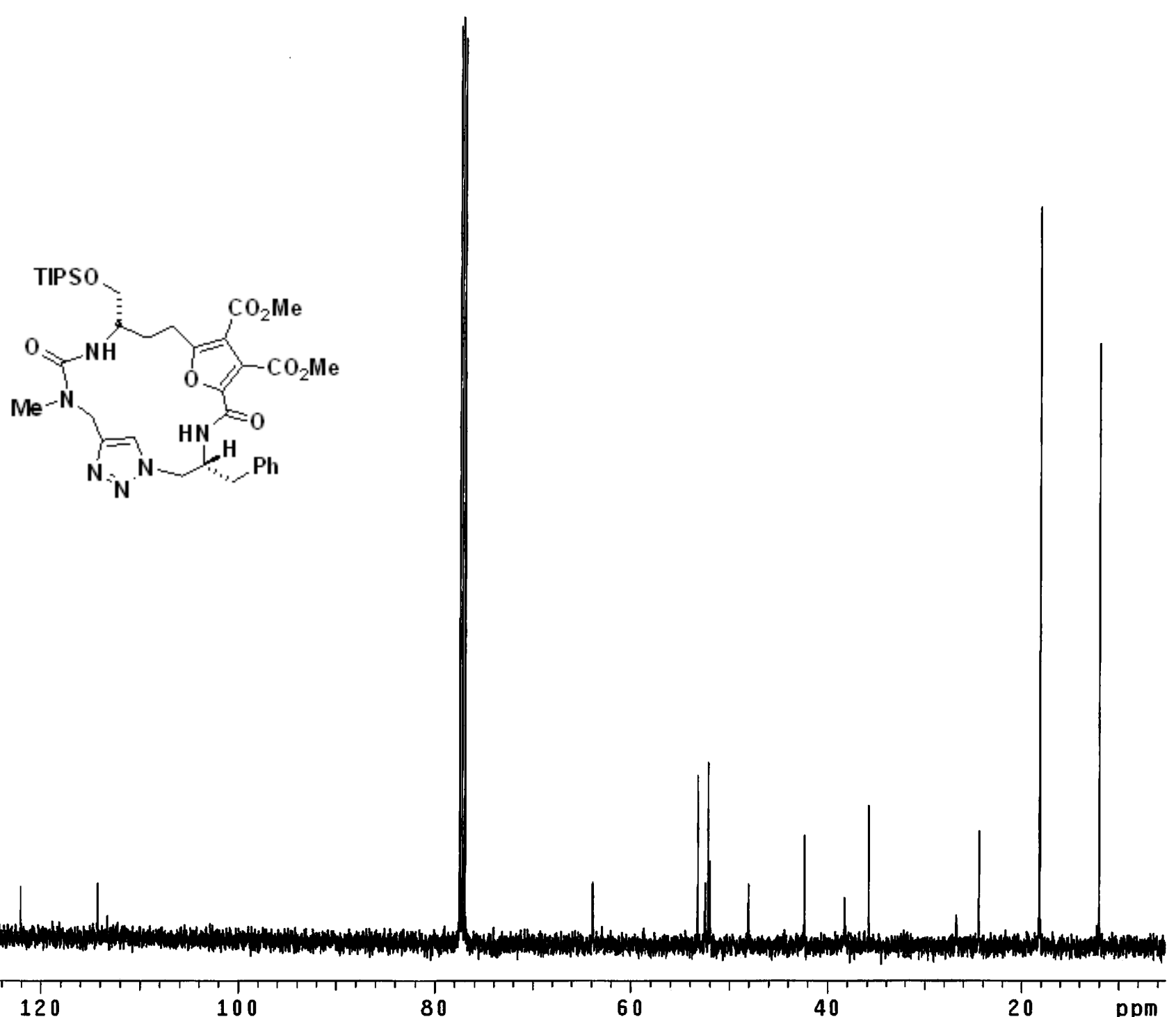


Pulse Sequence: s2pul

Solvent: $\operatorname{CDC} 13$

Temp. $23.0 \mathrm{C} / 296.1 \mathrm{~K}$

Pulse 77.1 degrees

Acq. time ${ }^{2} .{ }^{731}$

16 repetitions

OBSERVE H1, $399.8366318 \mathrm{MHz}$

DATA PROCESSIN

FT S1ze 32268 ing $0.1 \mathrm{~Hz}$

Total time $1 \mathrm{~min}, 2 \mathrm{sec}$

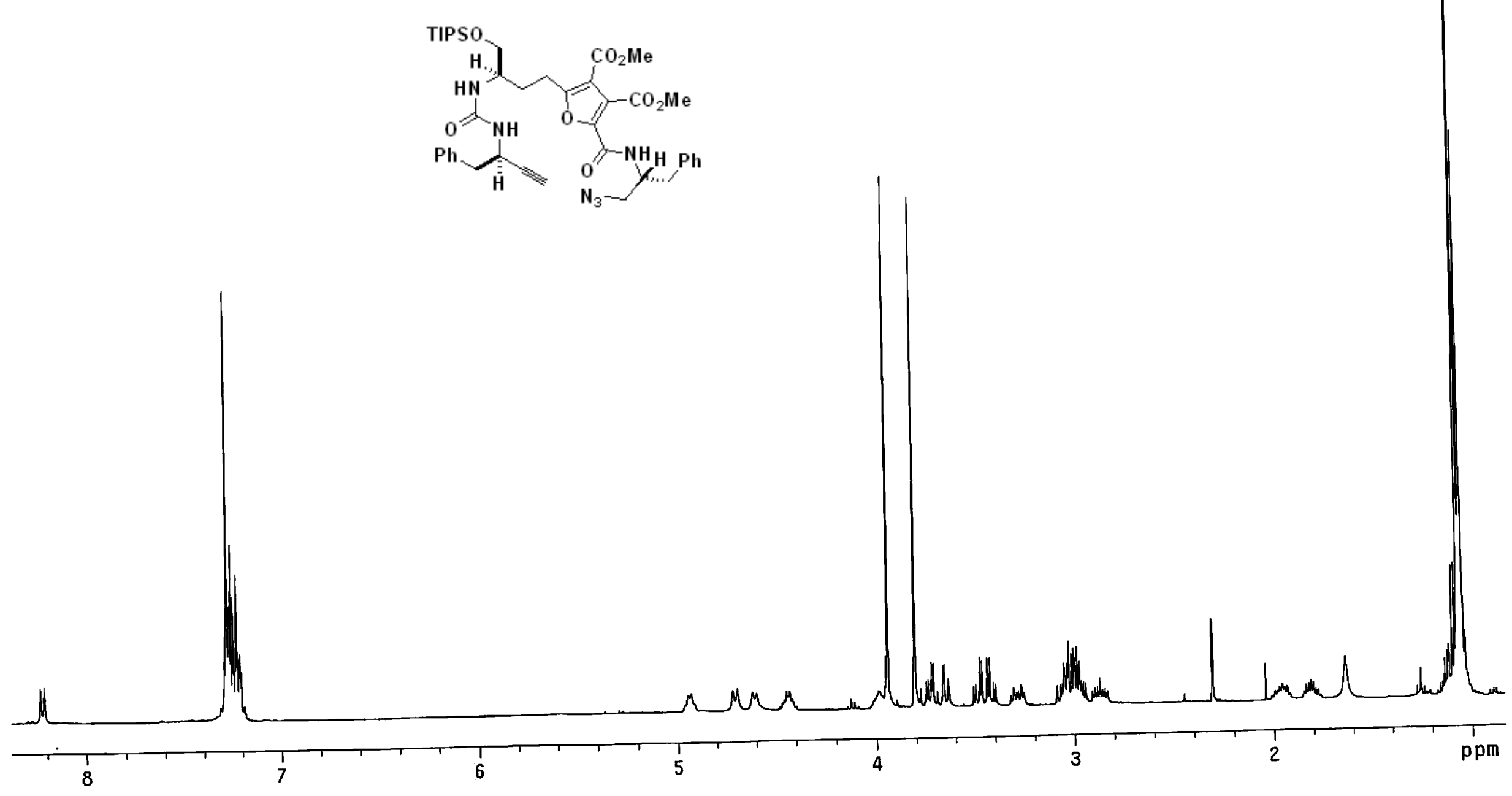


RELII099-RRR-C13

Pulse Sequence: s2pul

Solvent: $\operatorname{CDC} 13$

Temp. $23.0 \mathrm{C} / 296.1 \mathrm{~K}$
Mercury-400B

Pulse 36.7 degrees

Acq. time $1.311 \mathrm{se}$

Width $23980.8 \mathrm{~Hz}$

OBSERVE C13,100.5389786 MHZ

DECOUPLE H1, $399.8385865 \mathrm{MHZ}$

Power $47 \mathrm{~dB}$

continuous ly on

WALTZ-16 modu lated

Line broadening $1.0 \mathrm{~Hz}$

FT size 65536
Total time $28 \mathrm{~min}, 42 \mathrm{sec}$

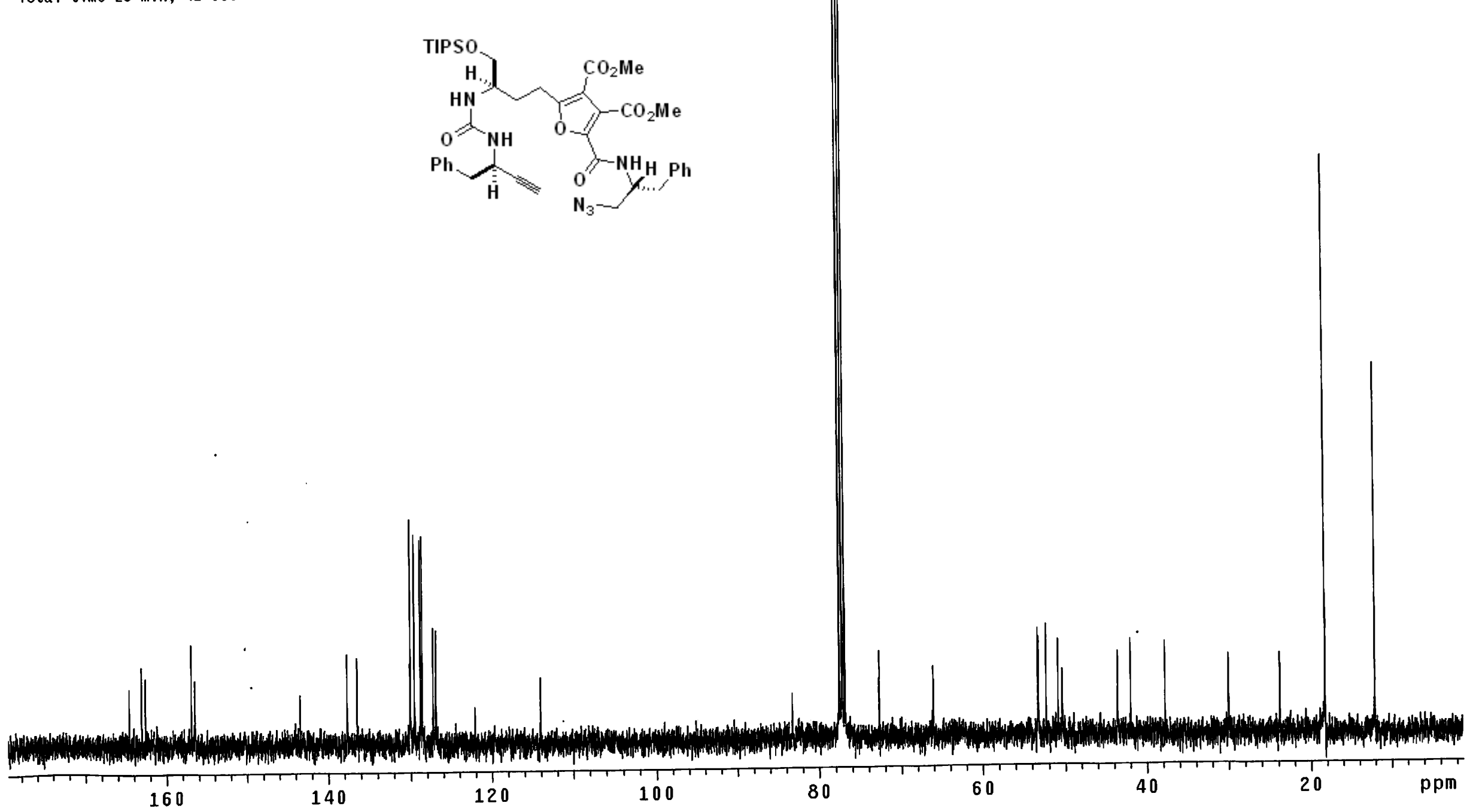




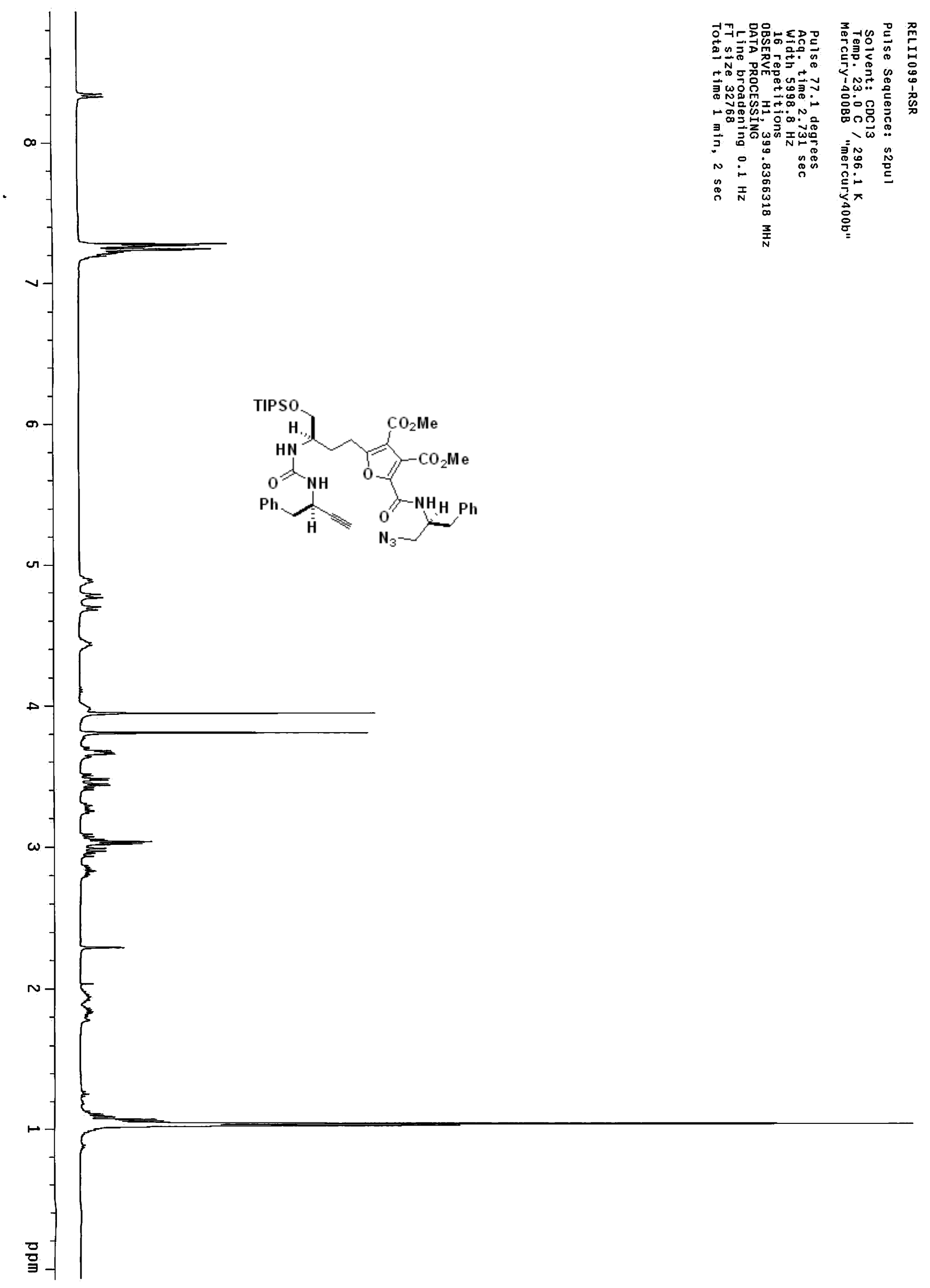


RELII 099 -RSR-C13

Pulse Sequence: s2pul

Solvent: $\operatorname{CDC} 13$

Temp. $23.0 \mathrm{C} / 296.1 \mathrm{~K}$
Mercury-400B
"mercury400b"

Pulse 36.7 degrees

Acq time $1.311 \mathrm{se}$

Width $23980.8 \mathrm{~Hz}$

192 repetitions

OBSERVE C13, 100.5389823 MHZ

Power 17 a 3 ,

cont inuous iy on

WALTZ-16 modulated

DATA PROCESSING

Line broadening $1.0 \mathrm{~Hz}$

Total time $28 \mathrm{~min}, 42 \mathrm{sec}$
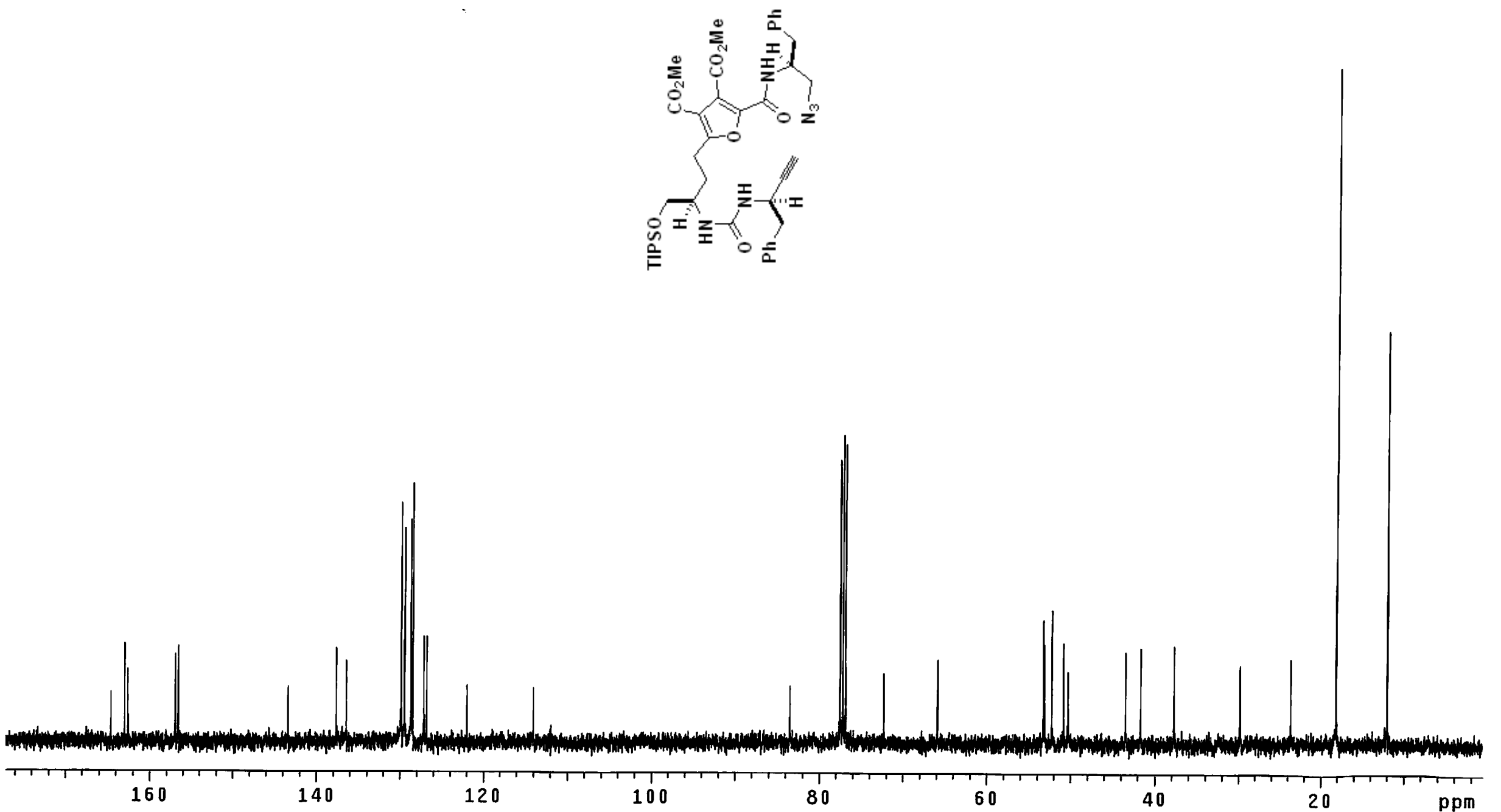


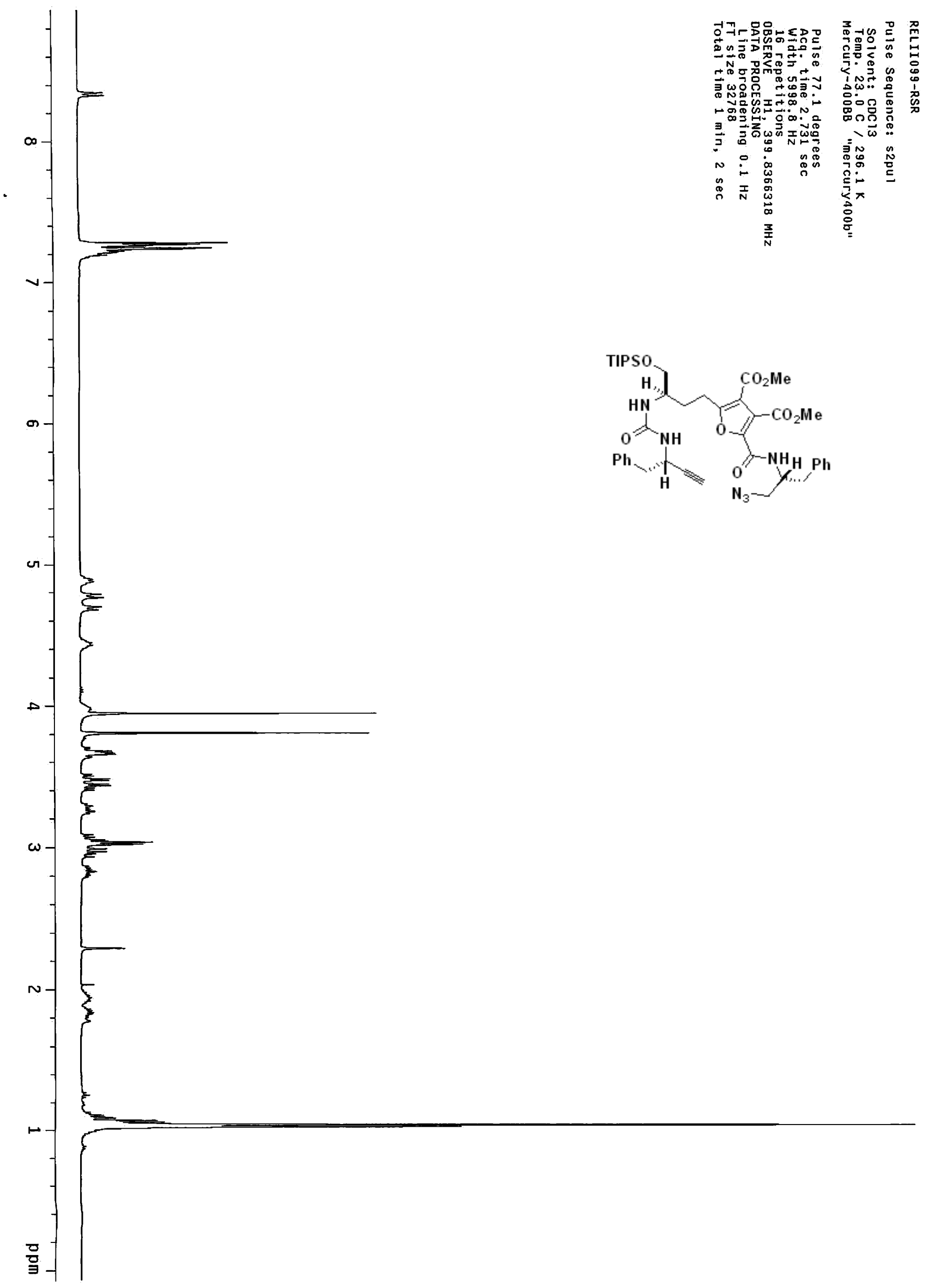


Pulse Sequence: $\$ 2 \mathrm{pul}$

Solvent: $\operatorname{CDC} 13$

Temp. $23.0 \mathrm{C} / 296.1 \mathrm{~K}$
Mercury-400B
"mercury400b"

Pulse 36.7 degrees

Acq time $1.311 \mathrm{se}$

Width $23980.8 \mathrm{~Hz}$

192 repetitions

OBSERVE C13, 100.5389823 MHZ

Power $17 \mathrm{~dB}$,

cont inuous iy on

WAL T2-16 modulated

DATA PROCESSING

LThe broadening $1.0 \mathrm{~Hz}$

Total time $28 \mathrm{~min}, 42 \mathrm{sec}$
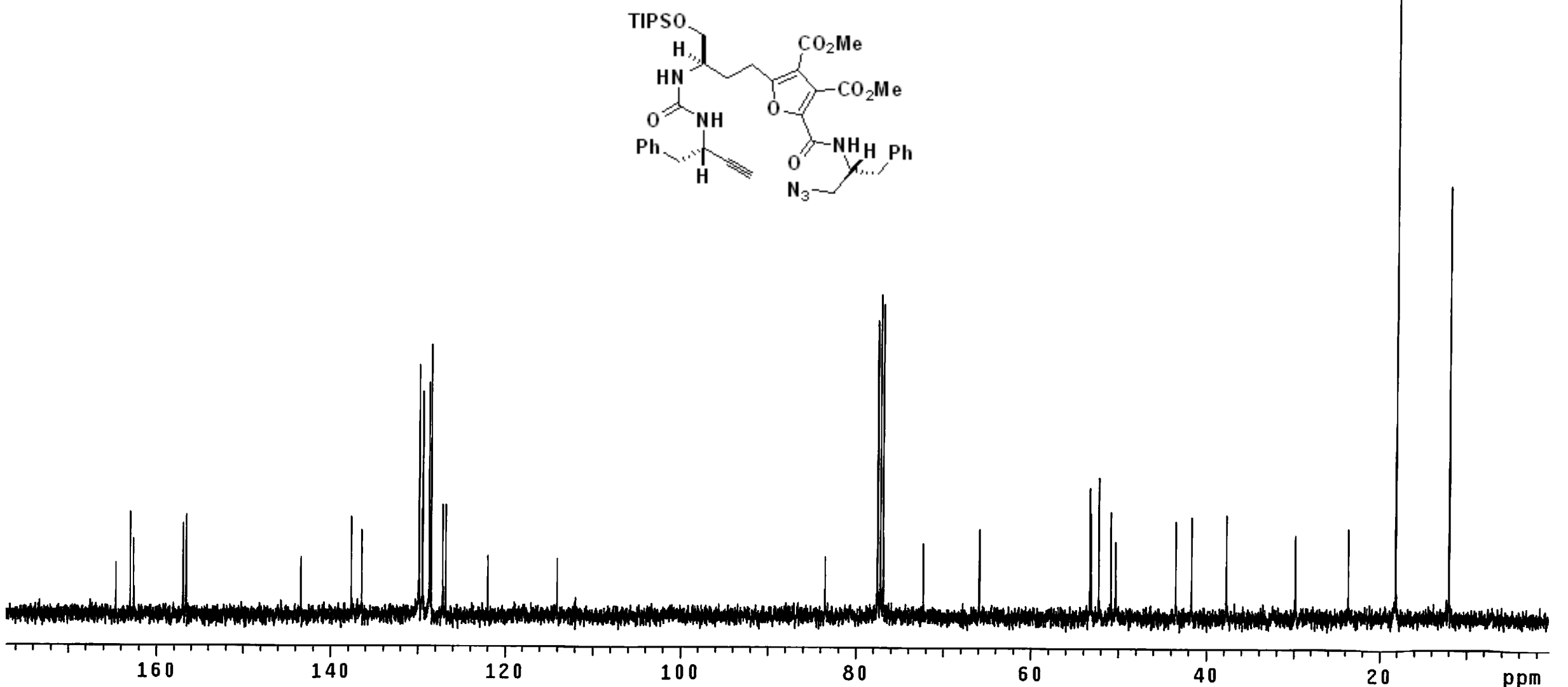
Pulse Sequence: s2pul

Solvent: $\operatorname{CDC}_{13}$

Temp. $23.0 \mathrm{C} / 296.1 \mathrm{~K}$
Mercury-400B

Pulse 77.1 degrees

Width $5998.8 \mathrm{~Hz}$

16 repetitions

OBSERVE H1, $399.8366318 \mathrm{MHZ}$

DATA PROCESSING $0.1 \mathrm{~Hz}$

FT size $32768 \mathrm{~min}, 2 \mathrm{sec}$
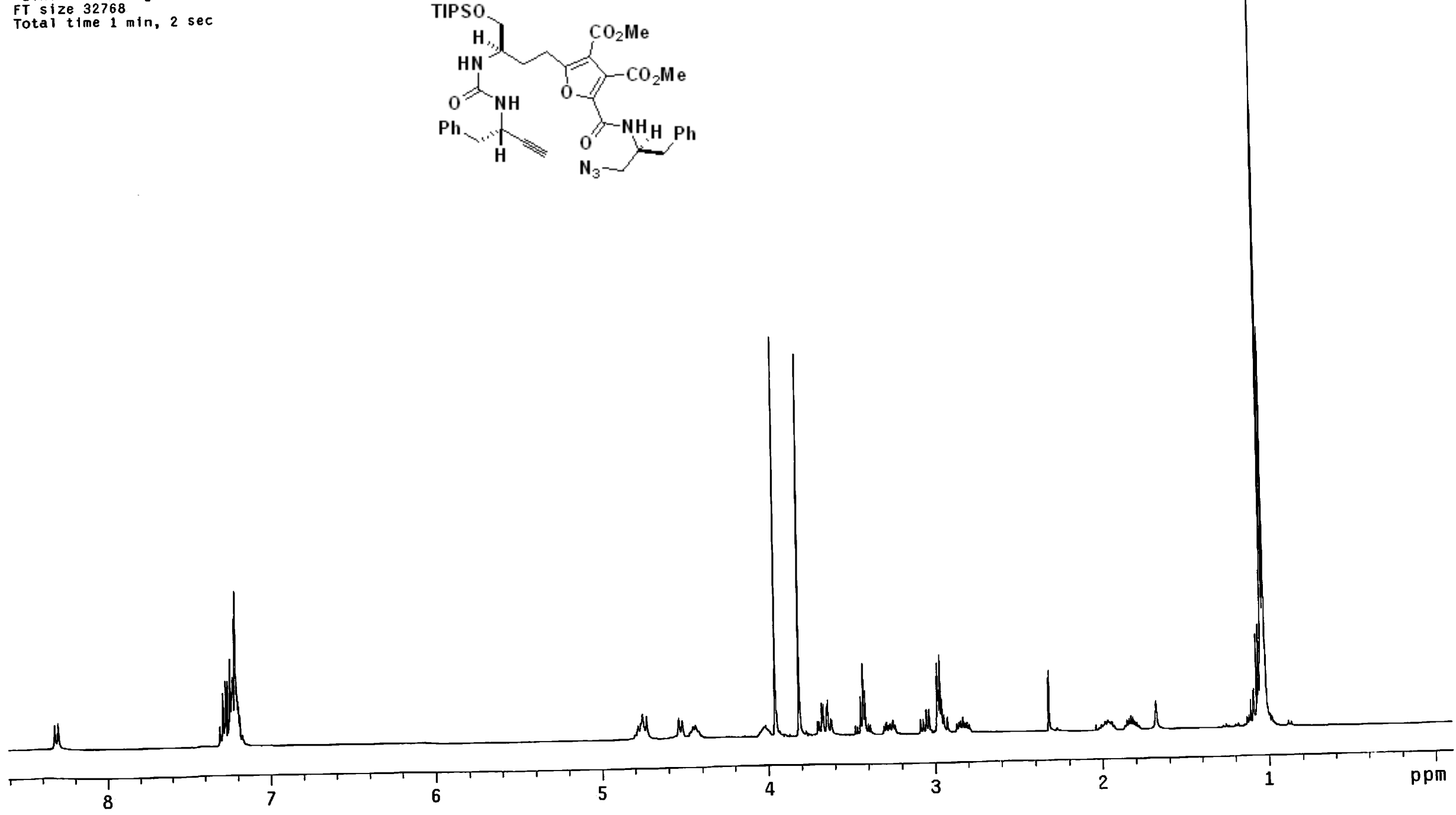
RELII099-RSS-C13

Pulse Sequence: s2pul

Solvent: $\operatorname{CDC} 13$

Temp. $23.0 \mathrm{C} / 296.1 \mathrm{~K}$
Mercury-400BB

Pulse 36.7 degrees

Acq. time 1.311 se

Width $23980.8 \mathrm{~Hz}$

OBSERVE C13,100.5389801 MH

DECOUPLE H1, 399.8385865 MHZ

Power 47 dB

cont inuous ly on

WALT2-16 modulated

line broadening

Total time $28 \mathrm{~min}, 42 \mathrm{sec}$
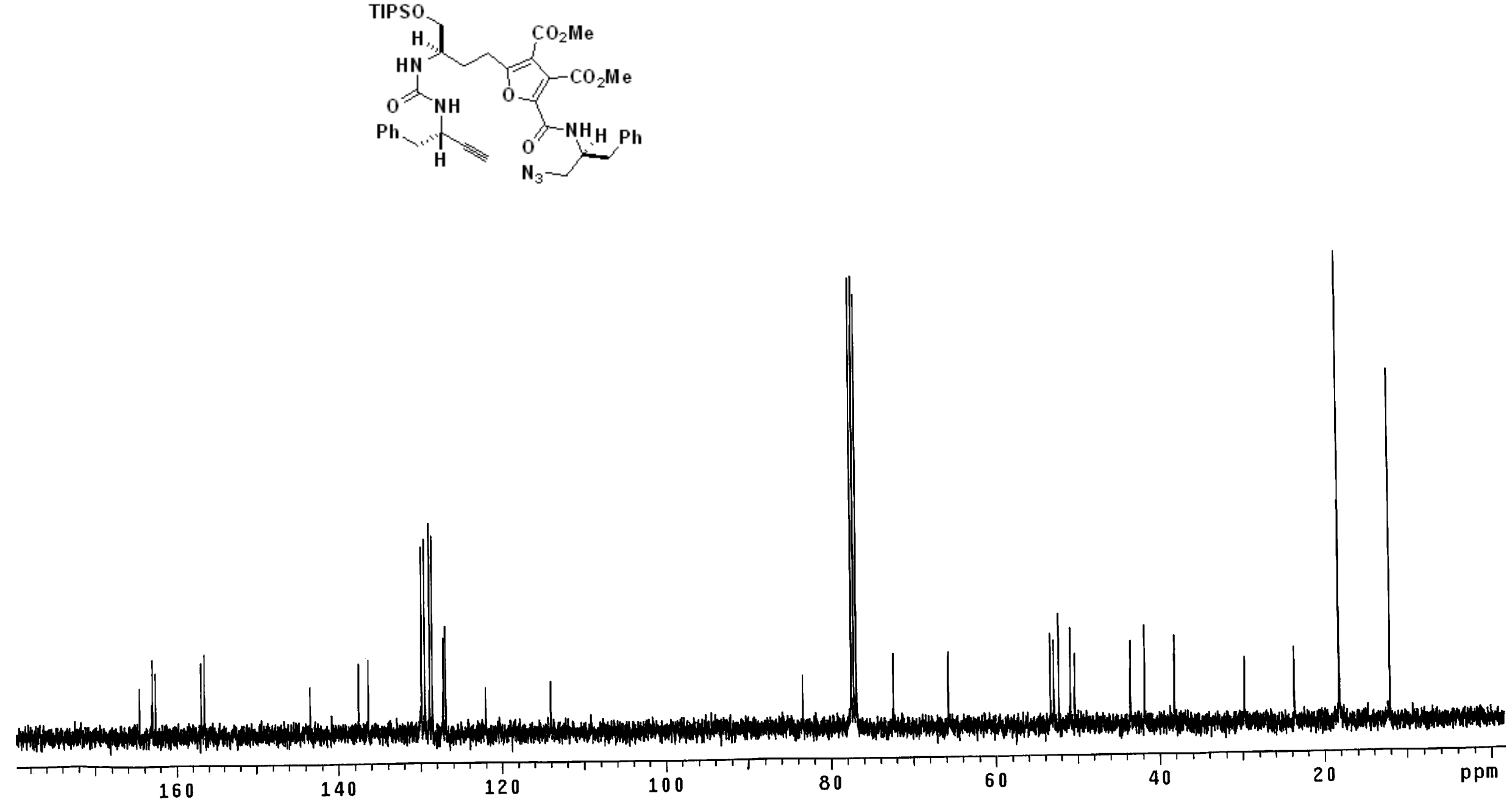
RELIII 100 -RRR-MCAR-MONOMER-MEOH

Pulse Sequence: s2pul

Temp. $23.0 \mathrm{C} / 296.1 \mathrm{~K}$
Mercury-4008B "mercury400b"

Pulse 77.1 degrees

Acq t ime 2.731 sec

Widtetions

28 repet tons $39.8382071 \mathrm{MHZ}$

DATA PROCESSING

Line broadening $0.1 \mathrm{~Hz}$

FT size 32768
Total time $1 \mathrm{~min}, 55 \mathrm{sec}$
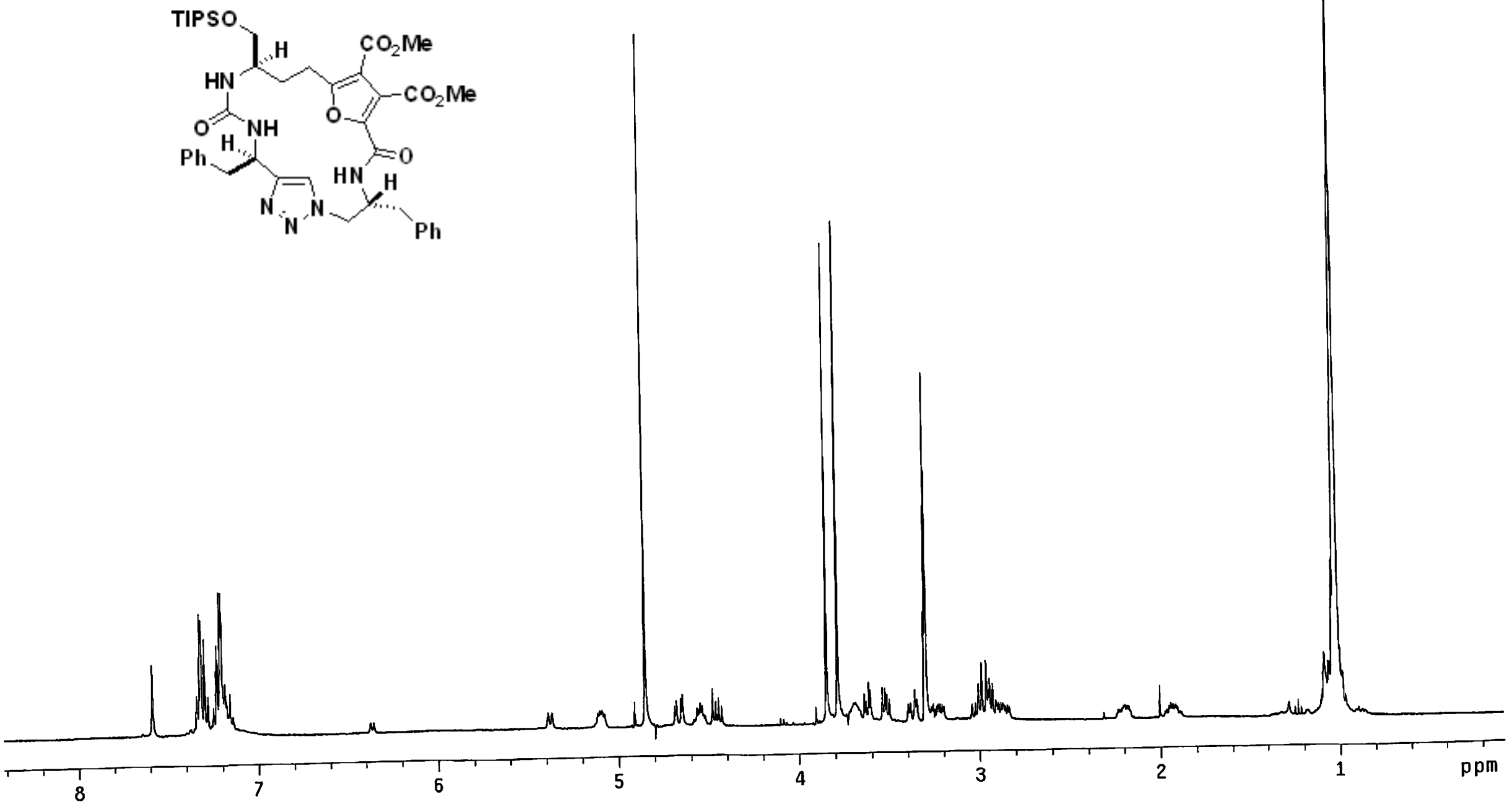

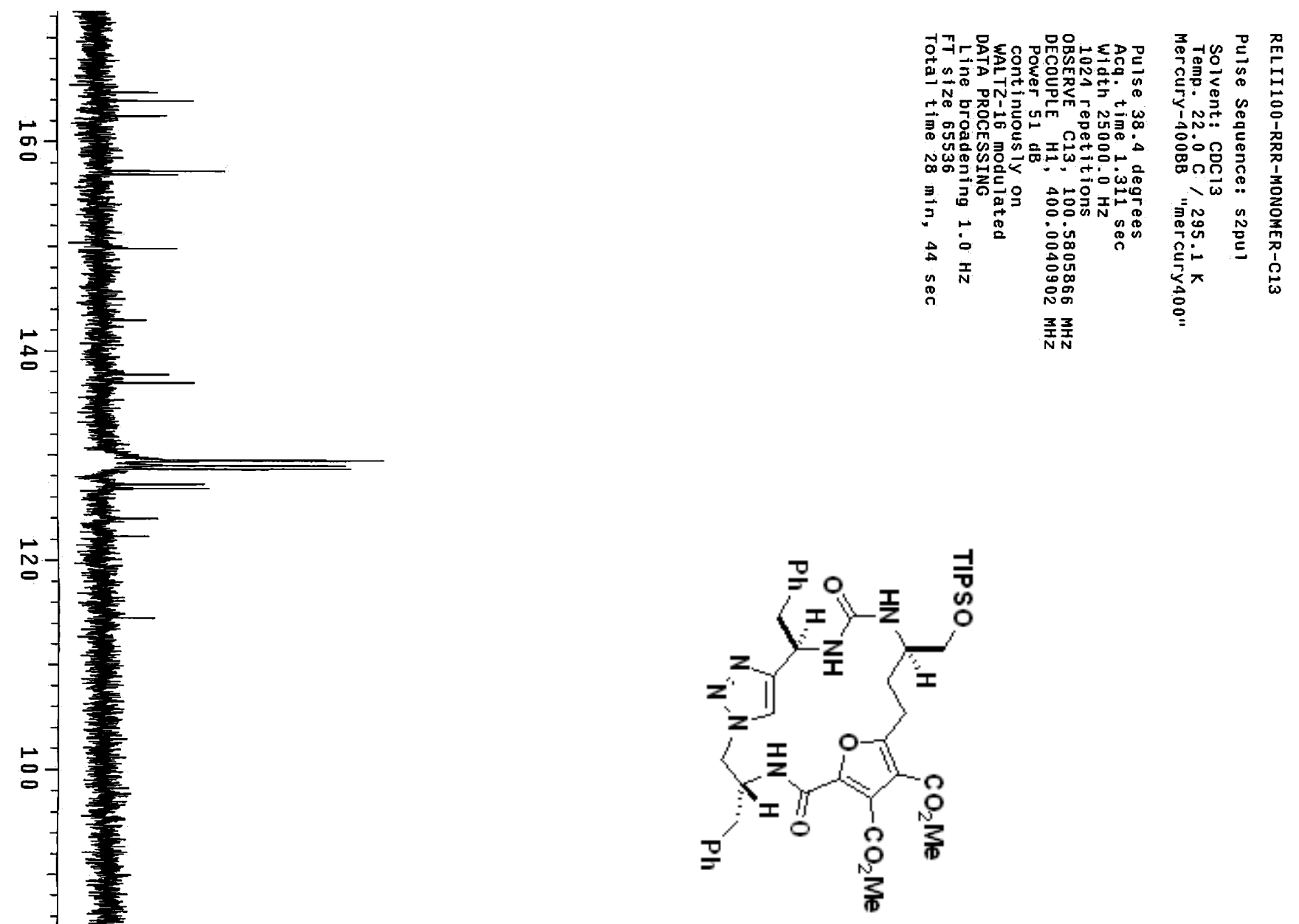

$\stackrel{\infty}{\circ}$

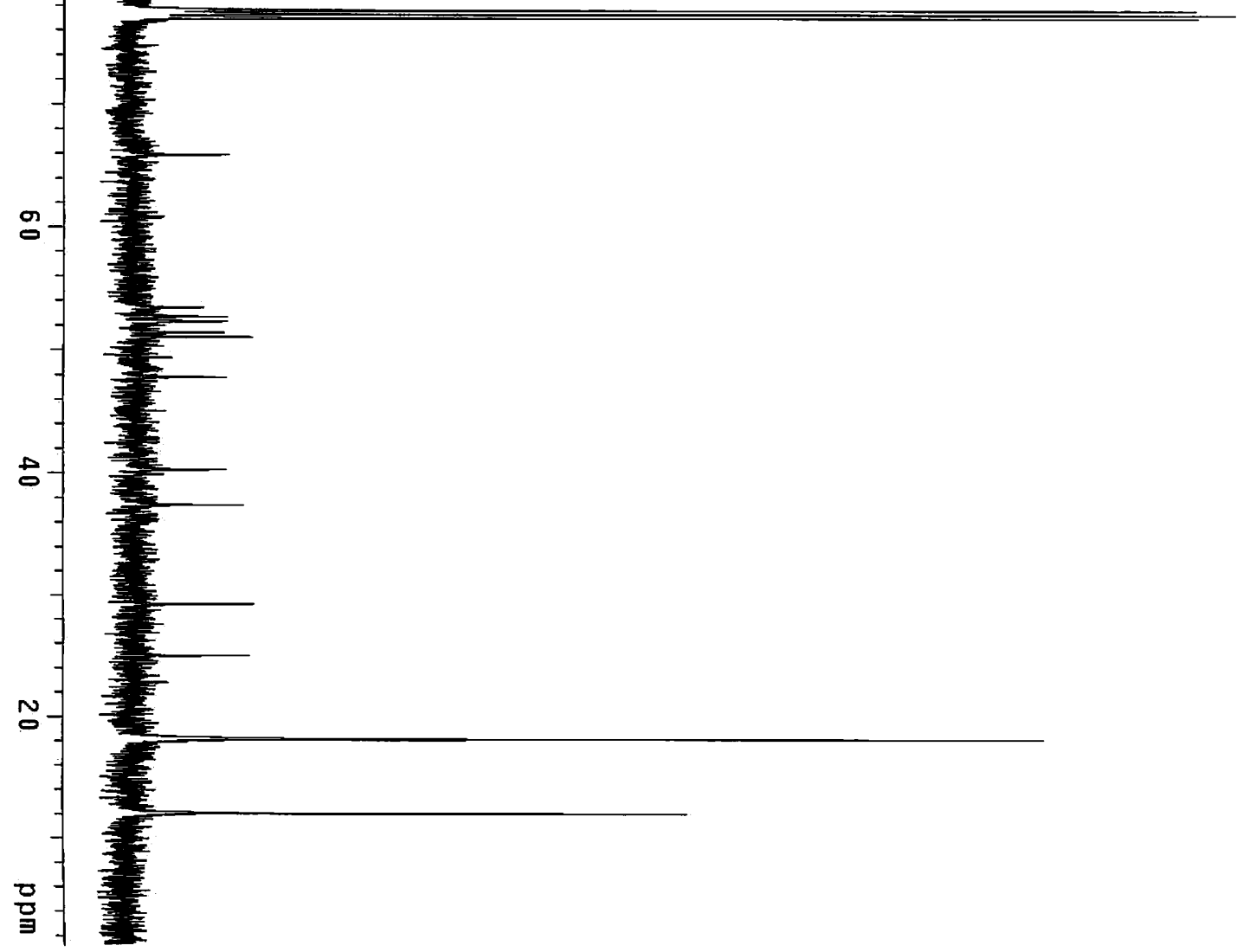


RELII 121-RRR-AMIDE

Pulse Sequence: s2pul

Solvent: $\mathrm{CDC}_{13}$

Temp. $23.00 \mathrm{C} / 296.1 \mathrm{~K}$
Mercury-400BB "mercury400b"

Pulse 77.1 degrees

Acq time 2.731 soc

Width $5998.8 \mathrm{~Hz}$

16 repetitions

DATA PROCESSING

Line broadening $0.1 \mathrm{~Hz}$

FT size 32768

total time $1 \mathrm{~min}, 2 \mathrm{sec}$

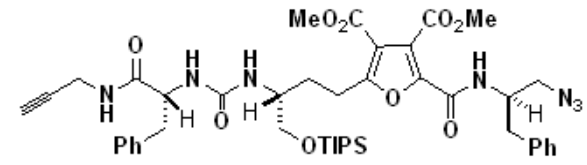

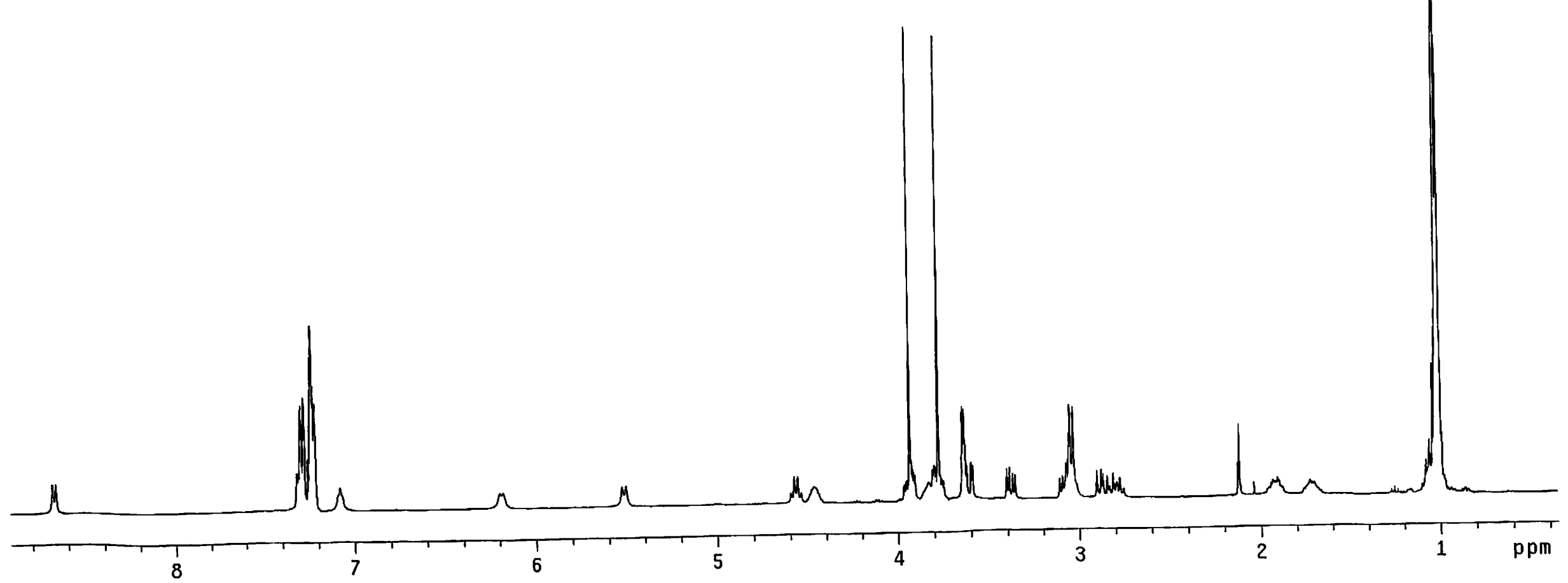


RELII 121-RRR-AMIDE-C13

Pulse Sequence: s2pul

Solvent: $\operatorname{CDC}_{13}$

Temp. $23.0 \mathrm{C} / 296.1 \mathrm{~K}$

Pulse 36.7 degrees

Acq. time 1.311 sec

192 repetitions

OBSERVE C13, 100.5389823 MHZ

DECOUPLE H1, $399.8385865 \mathrm{MHZ}$

Power $47 \mathrm{~dB}$

Cont inuous ly on

DATA PROCESSING

line broadening $1.0 \mathrm{~Hz}$

FT size 65536
Total time $28 \mathrm{~min}, 42 \mathrm{sec}$

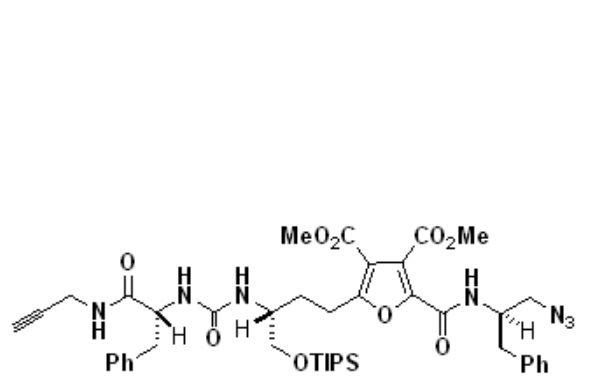

(1)

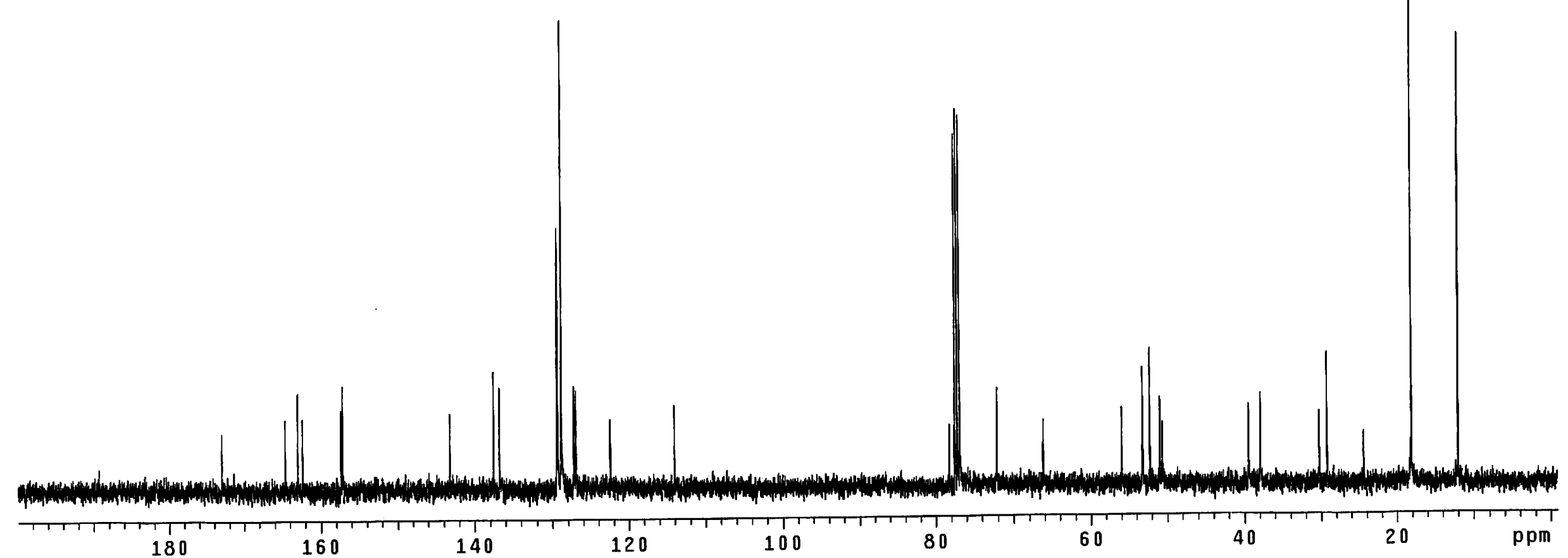


RELII121-RSS-AMIDE

Pulse Sequence: s2pul

Solvent: $\mathrm{CDC}_{13}$

Mercury-400B $22,05.1 \mathrm{~K}$

Re lax. de lay $0.120 \mathrm{sec}$

Pulse 64.3 degrees

Acq. time $2.731 \mathrm{sec}$

Width $5995.2 \mathrm{~Hz}$

OBSERVE H1, $400.0020964 \mathrm{MHZ}$

DATA PROCESSING

line broadening $0.1 \mathrm{~Hz}$

FT size 32768
Total time $1 \mathrm{~min}, 1 \mathrm{sec}$
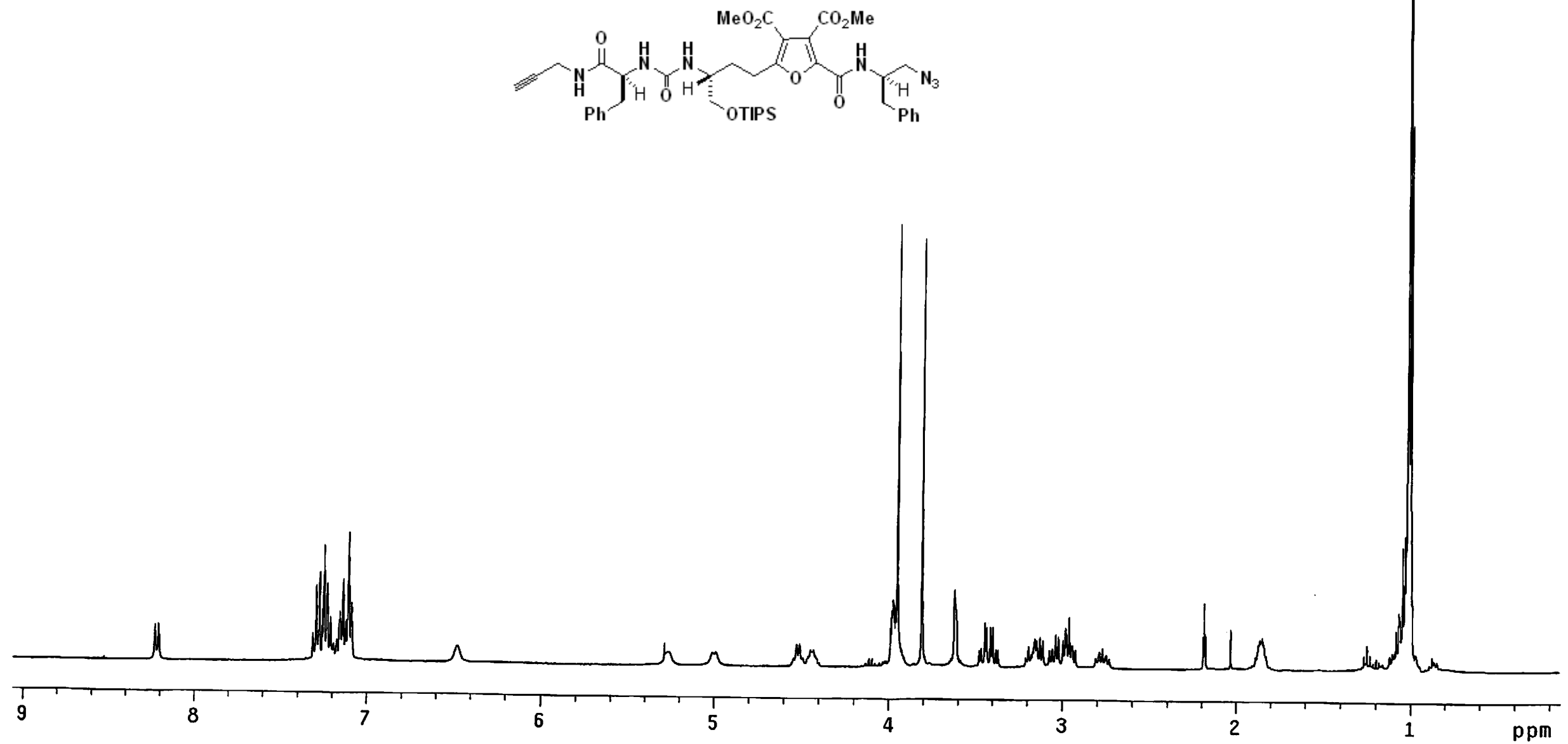
RELII121-RSS-AMIDE-C13

Pulse Sequence: s2pur

Solvent: $\operatorname{CDC} 13$

Temp. $22.0 \mathrm{C} / 295.1 \mathrm{~K}$

Pulse 38.4 degrees

Acq time $25000.3 \mathrm{~Hz}$

204 repetitions

OBSERVE C13, 100.5805881 $\mathrm{MH}$

DECOUPLE H1, 400.0040902 MH

Power 51 dB

WALTZ-16 modulated

DATA PROCESSING

line broadening $1.0 \mathrm{HZ}$

FT size 65536
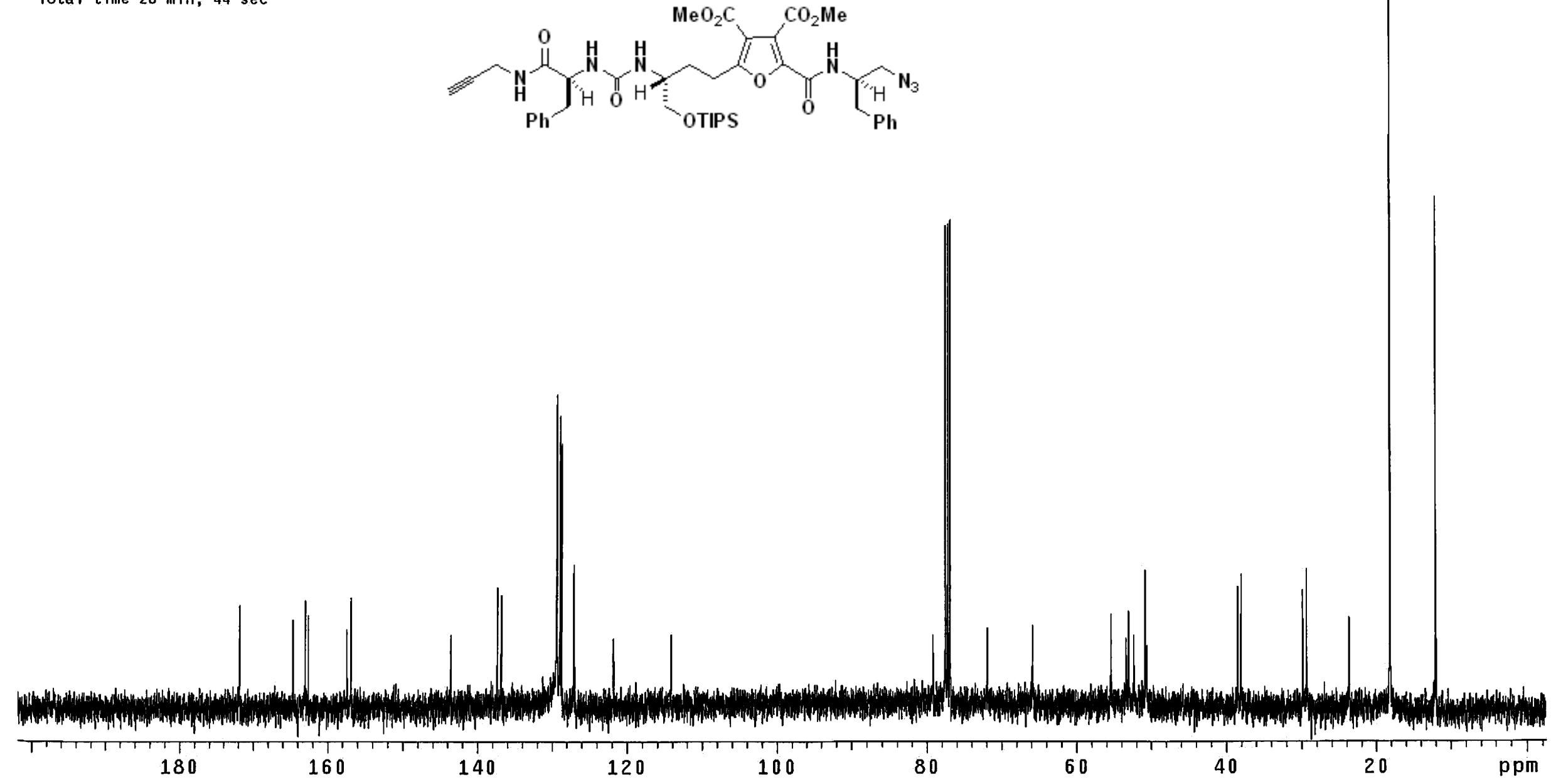
RELII-RSS-MCAR-AMIDE-70deg

Pulse Sequence: $\$ 2 p u 1$

Solvent: DMSO

Temp. $70.0 \mathrm{C} / 343.1 \mathrm{~K}$

Relax. delay $0.200 \mathrm{sec}$

Pulse 46.1 degrees

width $8000.0 \mathrm{~Hz}$

40 repetitions
OBSERVE H1, $499.7502831 \mathrm{MHz}$

DATA PROCESSII

FT size 32768 ing $0.1 \mathrm{~Hz}$

Total time $4 \mathrm{~min}, 53 \mathrm{sec}$
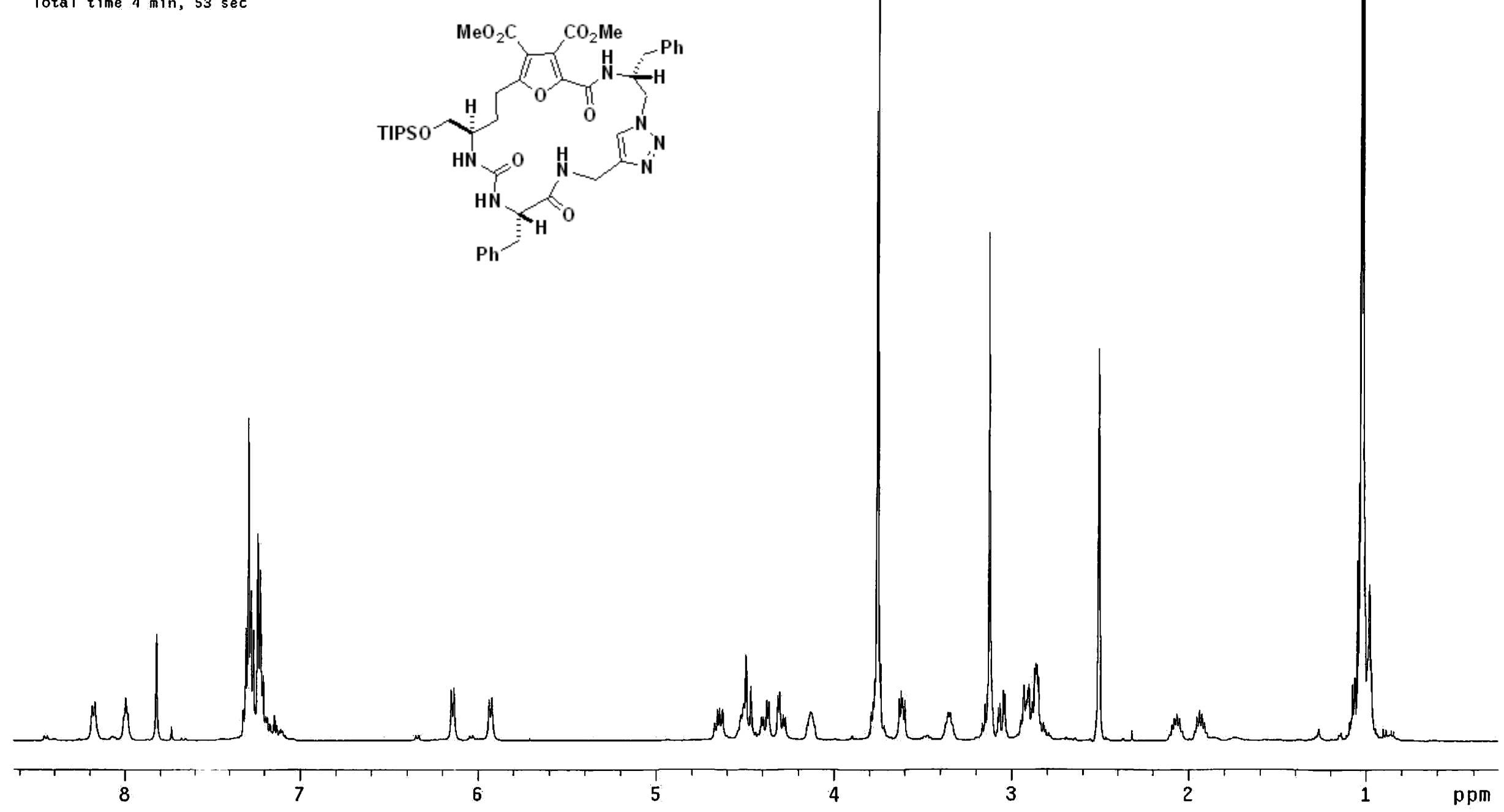
13C OBSERVE

Pulse Sequence: s2pur

Solvent: $\operatorname{CoC} 13$

Temp. $22.0 \mathrm{C} / 295.1 \mathrm{~K}$
Mercury-400BB

Pulse 38.4 degrees

Acq. time $1.311 \mathrm{sec}$

Width $25000 . \mathrm{Hz}$

OBSERVE C13, $100.5805889 \mathrm{MHZ}$

DECOUPLE H1, 400.0040902 MHZ

Power 51 dB

cont inuousiy on

DATA PROCESSING

line broadentng $1.0 \mathrm{~Hz}$

Total time $467100 \mathrm{hr}, 54 \mathrm{~min}, 21 \mathrm{sec}$
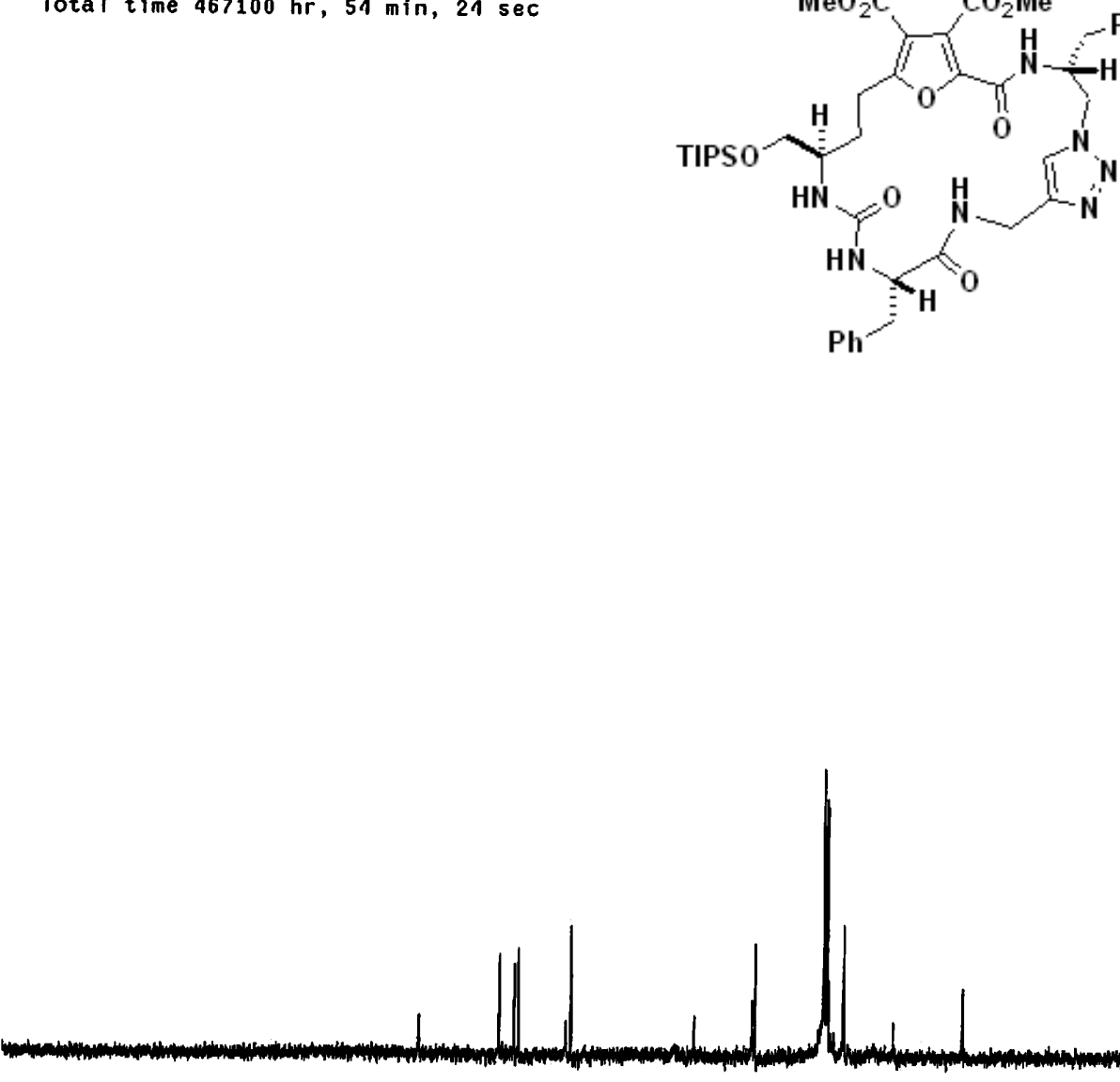
Structural Data

Date: July 1, 2005

Submitter: Ryan Looper

X-ray Crystallographic Laboratory

Harvard University

Sample Reference Number: RL1

Cambridge, MA 02138

X-ray Number: SLS87

Dr. Richard J. Staples

Staples@chemistry.harvard.edu

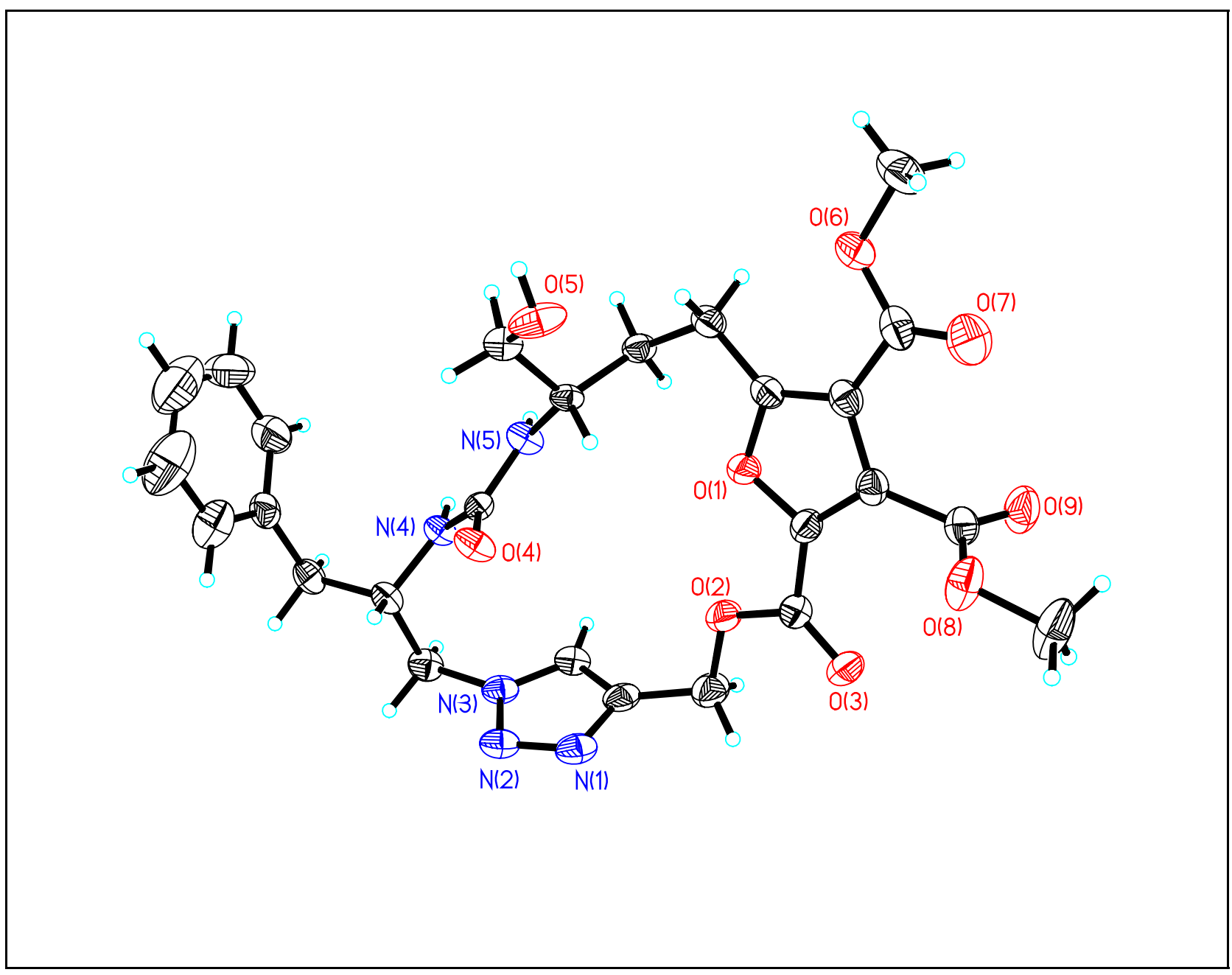

\section{Introduction:}

Single crystal study to confirm the identity of the sample submitted. 
Experimental Section:

A colorless thin needle crystal with dimensions $0.28 \times 0.08 \times 0.08 \mathrm{~mm}$ was mounted on a $0.2 \mathrm{~nm}$ nylon loop using very small amount of paratone oil.

Data were collected using a Bruker SMART APEX CCD (charge coupled device) based diffractometer equipped with an Oxford Cryostream low-temperature apparatus operating at $193 \mathrm{~K}$. Data were measured using omega scans of $0.3^{\circ}$ per frame for 30 seconds, such that a hemisphere was collected. A total of 1271 frames were collected with a maximum resolution of $0.76 \AA$. The first 50 frames were recollected at the end of data collection to monitor for decay. Cell parameters were retrieved using SMART $^{1}$ software and refined using SAINT on all observed reflections. Data reduction was performed using the SAINT software ${ }^{2}$ which corrects for Lp and decay. The structures are solved by the direct method using the SHELXS- $97^{3}$ program and refined by least squares method on $\mathrm{F}^{2}$, SHELXL-97, ${ }^{4}$ incorporated in SHELXTL-PC V 6.10.

The structure was solved in the space group $\mathrm{P} 2{ }_{1} 2{ }_{1} 2_{1}$ (\# 19) by analysis of systematic absences. All non-hydrogen atoms are refined anisotropically. Hydrogens were calculated by geometrical methods and refined as a riding model. The crystal used for the diffraction study showed no decomposition during data collection. All drawing are done at $50 \%$ ellipsoids.

Acknowledgement. The CCD based x-ray diffractometer at Harvard University was purchased through NIH grant (1S10RR11937-01). 


\section{References}

1. SMART V 5.625 (NT) Software for the CCD Detector System; Bruker Analytical X-ray Systems, Madison, WI (2001).

2. SAINT V 6.22 (NT) Software for the CCD Detector System Bruker Analytical Xray Systems, Madison, WI (2001).

3. Sheldrick, G. M. SHELXS-90, Program for the Solution of Crystal Structure, University of Göttingen, Germany, 1990.

4. Sheldrick, G. M. SHELXL-97, Program for the Refinement of Crystal Structure, University of Göttingen, Germany, 1997.

5. SHELXTL 6.1 (PC-Version), Program library for Structure Solution and Molecular Graphics; Bruker Analytical X-ray Systems, Madison, WI (2000) .

6. Flack, H. D.. Acta Cryst. A39, 1983, 876--881.

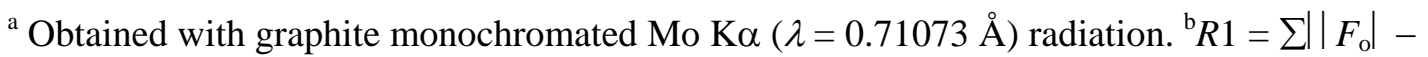
$\left|F_{\mathrm{c}}\right|\left|/ \sum\right| F_{\mathrm{o}} \mid .{ }^{\mathrm{c}} w R_{2}=\left\{\sum\left[w\left(F_{\mathrm{o}}{ }^{2}-F_{\mathrm{c}}{ }^{2}\right)^{2} /\left\{\sum\left[w\left(F_{\mathrm{o}}{ }^{2}\right)^{2}\right]\right\}^{1 / 2}\right.\right.$.

The following are 50\% thermal ellipsoidal drawings of the molecule in the asymmetric cell with various amount of labeling. 


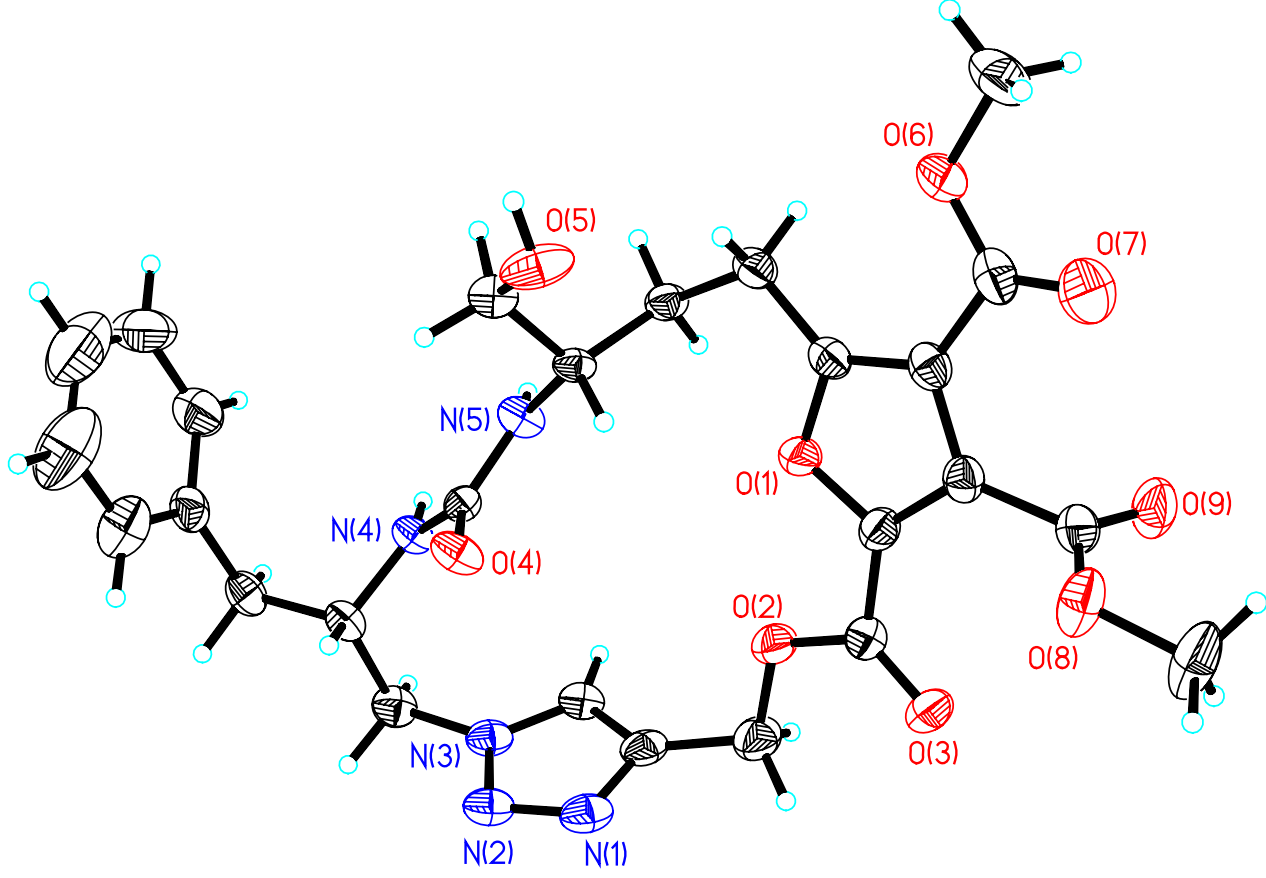




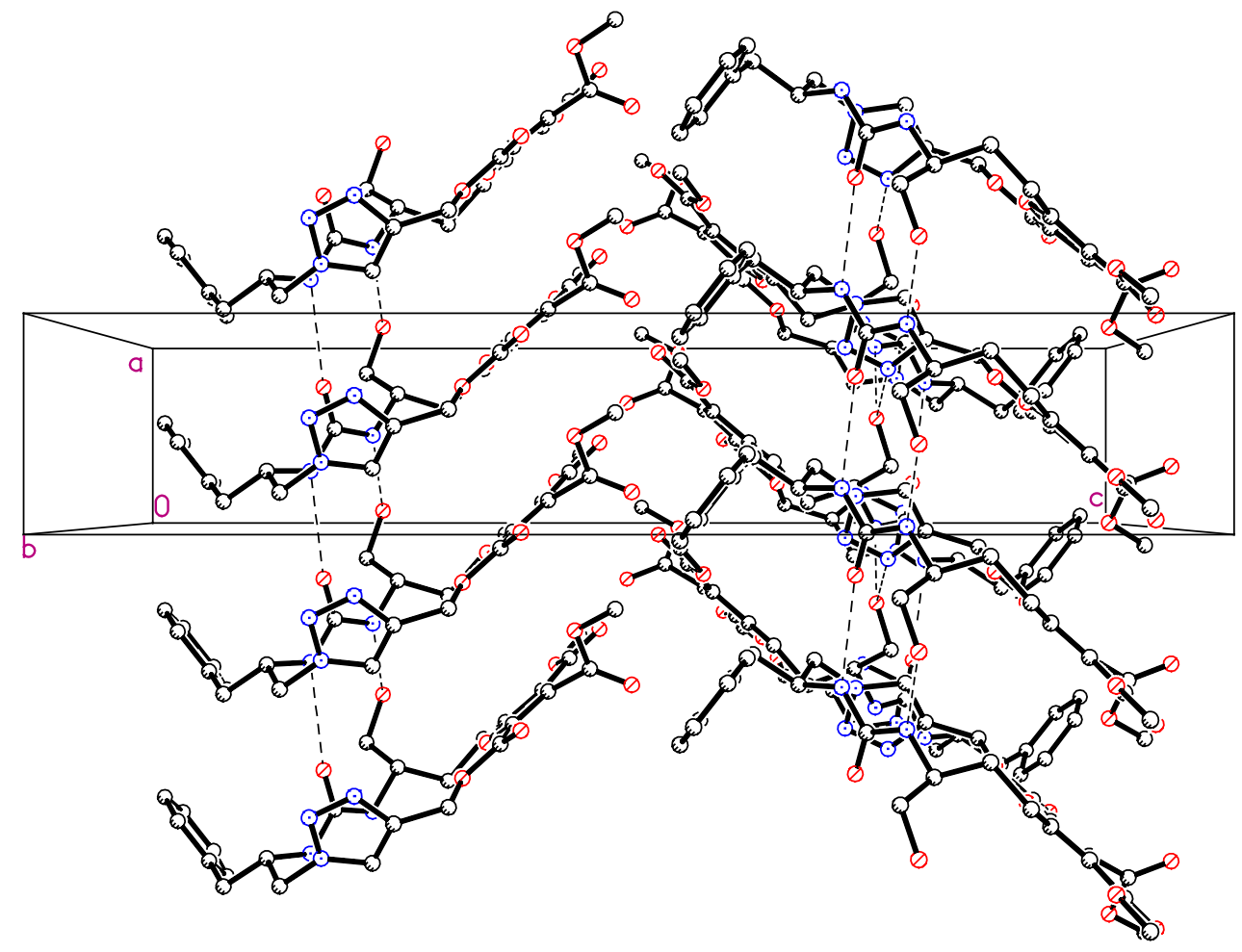

The last picture is the packing diagram along the b-axis which shows the best diagram of the hydrogen bonding within the crystal, dotted lines. 
Table 1. Crystal data and structure refinement for sls87.

Identification code

Empirical formula

Formula weight

Temperature

Wavelength

Crystal system

Space group

Unit cell dimensions

Volume

Z

Density (calculated)

Absorption coefficient

$\mathrm{F}(000)$

Crystal size

Theta range for data collection

Index ranges

Reflections collected

Independent reflections

Completeness to theta $=27.88^{\circ}$

Absorption correction

Refinement method

Data / restraints / parameters

Goodness-of-fit on $\mathrm{F}^{2}$

Final R indices [I $>2 \operatorname{sigma(I)]~}$

$\mathrm{R}$ indices (all data)

Absolute structure parameter

Largest diff. peak and hole sls87

C26 H29 N5 O9

555.54

193(2) K

$0.71073 \AA$

Orthorhombic

P2(1)2(1)2(1)

$\mathrm{a}=5.1429(10) \AA$

$\alpha=90^{\circ}$.

$\mathrm{b}=18.981(4) \AA$

$\beta=90^{\circ}$.

c $=28.092(6) \AA$

$\gamma=90^{\circ}$.

2742.3(10) $\AA^{3}$

4

$1.346 \mathrm{Mg} / \mathrm{m}^{3}$

$0.103 \mathrm{~mm}^{-1}$

1168

$0.26 \times 0.08 \times 0.08 \mathrm{~mm}^{3}$

1.45 to $27.88^{\circ}$.

$-6<=\mathrm{h}<=6,-24<=\mathrm{k}<=24,-24<=\mathrm{l}<=36$

19272

6506 [R(int) $=0.0562]$

$99.8 \%$

None

Full-matrix least-squares on $\mathrm{F}^{2}$

6506 / 0 / 364

1.003

$\mathrm{R} 1=0.0601, \mathrm{wR} 2=0.1126$

$\mathrm{R} 1=0.0967, \mathrm{wR} 2=0.1253$

$0.1(12)$

0.236 and -0.188 e. $\AA^{-3}$ 
Table 2. Atomic coordinates ( x $\left.10^{4}\right)$ and equivalent isotropic displacement parameters $\left(\AA^{2} \mathrm{x} 10^{3}\right)$ for sls87. $U(e q)$ is defined as one third of the trace of the orthogonalized $U^{i j}$ tensor.

\begin{tabular}{|c|c|c|c|c|}
\hline & $\mathrm{x}$ & $\mathrm{y}$ & $\mathrm{z}$ & $\mathrm{U}(\mathrm{eq})$ \\
\hline $\mathrm{O}(1)$ & $3641(4)$ & 1092(1) & 6367(1) & $32(1)$ \\
\hline $\mathrm{O}(2)$ & 2238(4) & $-168(1)$ & $6557(1)$ & $40(1)$ \\
\hline $\mathrm{O}(3)$ & 4821(4) & $-662(1)$ & 5999(1) & $39(1)$ \\
\hline $\mathrm{O}(4)$ & 2310(3) & 791(1) & 7919(1) & $32(1)$ \\
\hline $\mathrm{O}(5)$ & 5792(3) & 2597(1) & 7451(1) & $46(1)$ \\
\hline $\mathrm{O}(6)$ & 7388(4) & 2773(1) & 5733(1) & $44(1)$ \\
\hline $\mathrm{O}(7)$ & 9465(5) & 1968(1) & 5292(1) & $60(1)$ \\
\hline $\mathrm{O}(8)$ & $9728(4)$ & $98(1)$ & 5511(1) & $46(1)$ \\
\hline $\mathrm{O}(9)$ & 6841(4) & 484(1) & 4976(1) & $52(1)$ \\
\hline $\mathrm{N}(1)$ & 1684(4) & $-1108(1)$ & $7510(1)$ & $35(1)$ \\
\hline $\mathrm{N}(2)$ & $572(4)$ & $-1008(1)$ & 7927(1) & $33(1)$ \\
\hline $\mathrm{N}(3)$ & $-1633(4)$ & $-652(1)$ & 7843(1) & $30(1)$ \\
\hline $\mathrm{N}(4)$ & $-2059(4)$ & 830(1) & 8047(1) & $25(1)$ \\
\hline $\mathrm{N}(5)$ & $-165(4)$ & 1559(1) & 7495(1) & $28(1)$ \\
\hline $\mathrm{C}(1)$ & 4564(5) & 1716(1) & $6207(1)$ & $29(1)$ \\
\hline$C(2)$ & $6275(5)$ & 1597(1) & 5847(1) & $31(1)$ \\
\hline $\mathrm{C}(3)$ & $6408(5)$ & 855(1) & $5775(1)$ & $30(1)$ \\
\hline$C(4)$ & 4784(5) & $570(1)$ & 6104(1) & $30(1)$ \\
\hline$C(5)$ & 3988(5) & $-155(1)$ & 6203(1) & $31(1)$ \\
\hline$C(6)$ & 1031(6) & $-843(2)$ & $6659(1)$ & $42(1)$ \\
\hline$C(7)$ & $184(5)$ & $-816(1)$ & 7166(1) & $32(1)$ \\
\hline$C(8)$ & $-1933(5)$ & $-521(1)$ & 7378(1) & $32(1)$ \\
\hline $\mathrm{C}(9)$ & $-3159(5)$ & $-388(1)$ & $8246(1)$ & $33(1)$ \\
\hline$C(10)$ & $-2065(5)$ & $312(1)$ & 8423(1) & $26(1)$ \\
\hline$C(11)$ & $164(5)$ & 1047(1) & 7828(1) & $23(1)$ \\
\hline$C(12)$ & 2083(5) & 1888(1) & 7268(1) & $24(1)$ \\
\hline$C(13)$ & 1248(5) & 2196(1) & 6787(1) & $29(1)$ \\
\hline$C(14)$ & $3466(6)$ & 2355(1) & $6440(1)$ & $33(1)$ \\
\hline$C(15)$ & $7885(6)$ & 2121(2) & 5587(1) & $37(1)$ \\
\hline$C(16)$ & $8847(7)$ & 3331(2) & 5499(1) & $54(1)$ \\
\hline $\mathrm{C}(17)$ & 7684(5) & $465(2)$ & 5372(1) & $34(1)$ \\
\hline
\end{tabular}




$\begin{array}{lcrll}\mathrm{C}(18) & 10898(7) & -317(2) & 5130(1) & 59(1) \\ \mathrm{C}(19) & -3646(5) & 564(2) & 8855(1) & 33(1) \\ \mathrm{C}(20) & -2626(6) & 1242(2) & 9055(1) & 34(1) \\ \mathrm{C}(21) & -3742(8) & 1880(2) & 8943(1) & 61(1) \\ \mathrm{C}(22) & -2669(13) & 2511(2) & 9131(2) & 89(2) \\ \mathrm{C}(23) & -549(12) & 2471(3) & 9430(2) & 94(2) \\ \mathrm{C}(24) & 505(9) & 1844(3) & 9544(2) & 88(1) \\ \mathrm{C}(25) & -526(7) & 1246(2) & 9358(1) & 56(1) \\ \mathrm{C}(26) & 3231(5) & 2431(1) & 7604(1) & 31(1) \\ \end{array}$


Table 3. Bond lengths $[\AA]$ and angles $\left[{ }^{\circ}\right]$ for sls87.

\begin{tabular}{|c|c|c|c|}
\hline $\mathrm{O}(1)-\mathrm{C}(4)$ & $1.368(3)$ & $\mathrm{O}(1)-\mathrm{C}(1)$ & 1.353(3) \\
\hline $\mathrm{O}(2)-\mathrm{C}(6)$ & $1.452(3)$ & $\mathrm{O}(2)-\mathrm{C}(5)$ & 1.341(3) \\
\hline $\mathrm{O}(4)-\mathrm{C}(11)$ & $1.232(3)$ & $\mathrm{O}(3)-\mathrm{C}(5)$ & 1.198(3) \\
\hline $\mathrm{O}(5)-\mathrm{H}(5 \mathrm{~B})$ & 0.8400 & $\mathrm{O}(5)-\mathrm{C}(26)$ & $1.420(3)$ \\
\hline $\mathrm{O}(6)-\mathrm{C}(16)$ & $1.454(3)$ & $\mathrm{O}(6)-\mathrm{C}(15)$ & $1.329(3)$ \\
\hline $\mathrm{O}(8)-\mathrm{C}(17)$ & $1.320(3)$ & $\mathrm{O}(7)-\mathrm{C}(15)$ & 1.197(3) \\
\hline $\mathrm{O}(9)-\mathrm{C}(17)$ & $1.193(3)$ & $\mathrm{O}(8)-\mathrm{C}(18)$ & $1.459(3)$ \\
\hline $\mathrm{N}(1)-\mathrm{C}(7)$ & $1.355(3)$ & $\mathrm{N}(1)-\mathrm{N}(2)$ & 1.317(3) \\
\hline N(3)-C(8) & $1.340(3)$ & $\mathrm{N}(2)-\mathrm{N}(3)$ & $1.342(3)$ \\
\hline $\mathrm{N}(4)-\mathrm{C}(11)$ & $1.362(3)$ & $\mathrm{N}(3)-\mathrm{C}(9)$ & $1.467(3)$ \\
\hline $\mathrm{N}(4)-\mathrm{H}(4)$ & 0.8800 & $N(4)-C(10)$ & 1.442(3) \\
\hline N(5)-C(12) & $1.461(3)$ & $\mathrm{N}(5)-\mathrm{C}(11)$ & 1.361(3) \\
\hline$C(1)-C(2)$ & $1.360(4)$ & $\mathrm{N}(5)-\mathrm{H}(5)$ & 0.8800 \\
\hline $\mathrm{C}(2)-\mathrm{C}(3)$ & $1.424(4)$ & $C(1)-C(14)$ & $1.490(4)$ \\
\hline $\mathrm{C}(3)-\mathrm{C}(4)$ & $1.359(4)$ & $C(2)-C(15)$ & $1.486(4)$ \\
\hline$C(4)-C(5)$ & $1.463(4)$ & $\mathrm{C}(3)-\mathrm{C}(17)$ & $1.503(4)$ \\
\hline $\mathrm{C}(6)-\mathrm{H}(6 \mathrm{~A})$ & 0.9900 & $\mathrm{C}(6)-\mathrm{C}(7)$ & 1.491(4) \\
\hline C(7)-C(8) & $1.360(4)$ & $\mathrm{C}(6)-\mathrm{H}(6 \mathrm{~B})$ & 0.9900 \\
\hline C(9)-C(10) & $1.525(3)$ & $\mathrm{C}(8)-\mathrm{H}(8)$ & 0.9500 \\
\hline C(9)-H(9B) & 0.9900 & $\mathrm{C}(9)-\mathrm{H}(9 \mathrm{~A})$ & 0.9900 \\
\hline $\mathrm{C}(10)-\mathrm{H}(10)$ & 1.0000 & $\mathrm{C}(10)-\mathrm{C}(19)$ & $1.537(3)$ \\
\hline $\mathrm{C}(12)-\mathrm{C}(13)$ & $1.532(3)$ & $\mathrm{C}(12)-\mathrm{C}(26)$ & $1.516(3)$ \\
\hline C(13)-C(14) & $1.530(4)$ & $\mathrm{C}(12)-\mathrm{H}(12)$ & 1.0000 \\
\hline C(13)-H(13B) & 0.9900 & $\mathrm{C}(13)-\mathrm{H}(13 \mathrm{~A})$ & 0.9900 \\
\hline $\mathrm{C}(14)-\mathrm{H}(14 \mathrm{~B})$ & 0.9900 & $\mathrm{C}(14)-\mathrm{H}(14 \mathrm{~A})$ & 0.9900 \\
\hline C(16)-H(16B) & 0.9800 & $\mathrm{C}(16)-\mathrm{H}(16 \mathrm{~A})$ & 0.9800 \\
\hline $\mathrm{C}(18)-\mathrm{H}(18 \mathrm{~A})$ & 0.9800 & $\mathrm{C}(16)-\mathrm{H}(16 \mathrm{C})$ & 0.9800 \\
\hline C(18)-H(18C) & 0.9800 & $\mathrm{C}(18)-\mathrm{H}(18 \mathrm{~B})$ & 0.9800 \\
\hline C(19)-H(19A) & 0.9900 & $\mathrm{C}(19)-\mathrm{C}(20)$ & 1.499(4) \\
\hline C(20)-C(25) & $1.375(4)$ & $\mathrm{C}(19)-\mathrm{H}(19 \mathrm{~B})$ & 0.9900 \\
\hline$C(21)-C(22)$ & $1.420(6)$ & $C(20)-C(21)$ & $1.376(4)$ \\
\hline C(22)-C(23) & $1.378(7)$ & $\mathrm{C}(21)-\mathrm{H}(21)$ & 0.9500 \\
\hline C(23)-C(24) & $1.348(7)$ & $\mathrm{C}(22)-\mathrm{H}(22)$ & 0.9500 \\
\hline C(24)-C(25) & $1.358(5)$ & C(23)-H(23) & 0.9500 \\
\hline
\end{tabular}


$\mathrm{C}(25)-\mathrm{H}(25)$

C(26)-H(26B)

$\mathrm{C}(1)-\mathrm{O}(1)-\mathrm{C}(4)$

$\mathrm{C}(26)-\mathrm{O}(5)-\mathrm{H}(5 \mathrm{~B})$

$\mathrm{C}(17)-\mathrm{O}(8)-\mathrm{C}(18)$

$\mathrm{N}(1)-\mathrm{N}(2)-\mathrm{N}(3)$

$\mathrm{C}(8)-\mathrm{N}(3)-\mathrm{C}(9)$

$\mathrm{C}(11)-\mathrm{N}(4)-\mathrm{C}(10)$

$\mathrm{C}(10)-\mathrm{N}(4)-\mathrm{H}(4)$

$\mathrm{C}(11)-\mathrm{N}(5)-\mathrm{H}(5)$

$\mathrm{O}(1)-\mathrm{C}(1)-\mathrm{C}(2)$

C(2)-C(1)-C(14)

$\mathrm{C}(1)-\mathrm{C}(2)-\mathrm{C}(15)$

$\mathrm{C}(4)-\mathrm{C}(3)-\mathrm{C}(2)$

$\mathrm{C}(2)-\mathrm{C}(3)-\mathrm{C}(17)$

$\mathrm{C}(3)-\mathrm{C}(4)-\mathrm{C}(5)$

$\mathrm{O}(3)-\mathrm{C}(5)-\mathrm{O}(2)$

$\mathrm{O}(2)-\mathrm{C}(5)-\mathrm{C}(4)$

$\mathrm{O}(2)-\mathrm{C}(6)-\mathrm{H}(6 \mathrm{~A})$

$\mathrm{O}(2)-\mathrm{C}(6)-\mathrm{H}(6 \mathrm{~B})$

$\mathrm{H}(6 \mathrm{~A})-\mathrm{C}(6)-\mathrm{H}(6 \mathrm{~B})$

$\mathrm{N}(1)-\mathrm{C}(7)-\mathrm{C}(6)$

$\mathrm{N}(3)-\mathrm{C}(8)-\mathrm{C}(7)$

$\mathrm{C}(7)-\mathrm{C}(8)-\mathrm{H}(8)$

$\mathrm{N}(3)-\mathrm{C}(9)-\mathrm{H}(9 \mathrm{~A})$

$\mathrm{N}(3)-\mathrm{C}(9)-\mathrm{H}(9 \mathrm{~B})$

H(9A)-C(9)-H(9B)

N(4)-C(10)-C(19)

N(4)-C(10)-H(10)

C(19)-C(10)-H(10)

$\mathrm{O}(4)-\mathrm{C}(11)-\mathrm{N}(4)$

N(5)-C(12)-C(26)

C(26)-C(12)-C(13)
0.9500

0.9900

C(24)-H(24)

C(26)-H(26A)

0.9500

0.9900
107.7(2)

109.5

113.3(2)

106.4(2)

129.0(2)

122.5(2)

118.7

119.7

109.2(2)

135.0(2)

127.9(3)

105.4(2)

128.0(2)

132.5(2)

125.5(3)

110.2(2)

110.4

110.4

108.6

120.1(2)

104.9(2)

127.5

109.5

109.5

108.1

111.5(2)

108.3

108.3

122.6(2)

109.09(19)

113.5(2)
$\mathrm{C}(5)-\mathrm{O}(2)-\mathrm{C}(6)$

$\mathrm{C}(15)-\mathrm{O}(6)-\mathrm{C}(16)$

$\mathrm{N}(2)-\mathrm{N}(1)-\mathrm{C}(7)$

$\mathrm{C}(8)-\mathrm{N}(3)-\mathrm{N}(2)$

$\mathrm{N}(2)-\mathrm{N}(3)-\mathrm{C}(9)$

$\mathrm{C}(11)-\mathrm{N}(4)-\mathrm{H}(4)$

C(11)-N(5)-C(12)

$\mathrm{C}(12)-\mathrm{N}(5)-\mathrm{H}(5)$

$\mathrm{O}(1)-\mathrm{C}(1)-\mathrm{C}(14)$

$\mathrm{C}(1)-\mathrm{C}(2)-\mathrm{C}(3)$

$\mathrm{C}(3)-\mathrm{C}(2)-\mathrm{C}(15)$

$\mathrm{C}(4)-\mathrm{C}(3)-\mathrm{C}(17)$

$\mathrm{C}(3)-\mathrm{C}(4)-\mathrm{O}(1)$

$\mathrm{O}(1)-\mathrm{C}(4)-\mathrm{C}(5)$

$\mathrm{O}(3)-\mathrm{C}(5)-\mathrm{C}(4)$

$\mathrm{O}(2)-\mathrm{C}(6)-\mathrm{C}(7)$

$\mathrm{C}(7)-\mathrm{C}(6)-\mathrm{H}(6 \mathrm{~A})$

$\mathrm{C}(7)-\mathrm{C}(6)-\mathrm{H}(6 \mathrm{~B})$

$\mathrm{N}(1)-\mathrm{C}(7)-\mathrm{C}(8)$

$\mathrm{C}(8)-\mathrm{C}(7)-\mathrm{C}(6)$

$\mathrm{N}(3)-\mathrm{C}(8)-\mathrm{H}(8)$

N(3)-C(9)-C(10)

C(10)-C(9)-H(9A)

C(10)-C(9)-H(9B)

N(4)-C(10)-C(9)

C(9)-C(10)-C(19)

C(9)-C(10)-H(10)

$\mathrm{O}(4)-\mathrm{C}(11)-\mathrm{N}(5)$

$\mathrm{N}(5)-\mathrm{C}(11)-\mathrm{N}(4)$

N(5)-C(12)-C(13)

$\mathrm{N}(5)-\mathrm{C}(12)-\mathrm{H}(12)$
116.7(2)

116.1(2)

109.1(2)

111.3(2)

119.2(2)

118.7

120.5(2)

119.7

115.7(2)

107.6(2)

124.4(2)

125.8(2)

110.1(2)

117.3(2)

124.3(3)

106.5(2)

110.4

110.4

108.2(2)

131.7(3)

127.5

110.5(2)

109.5

109.5

110.9(2)

109.4(2)

108.3

122.4(2)

115.0(2)

109.0(2)

108.4 


\begin{tabular}{|c|c|c|c|}
\hline $\mathrm{C}(26)-\mathrm{C}(12)-\mathrm{H}(12)$ & 108.4 & $\mathrm{C}(13)-\mathrm{C}(12)-\mathrm{H}(12)$ & 108.4 \\
\hline$C(14)-C(13)-C(12)$ & $115.3(2)$ & $\mathrm{C}(14)-\mathrm{C}(13)-\mathrm{H}(13 \mathrm{~A})$ & 108.5 \\
\hline C(12)-C(13)-H(13A) & 108.5 & $\mathrm{C}(14)-\mathrm{C}(13)-\mathrm{H}(13 \mathrm{~B})$ & 108.5 \\
\hline $\mathrm{C}(12)-\mathrm{C}(13)-\mathrm{H}(13 \mathrm{~B})$ & 108.5 & $\mathrm{H}(13 \mathrm{~A})-\mathrm{C}(13)-\mathrm{H}(13 \mathrm{~B})$ & 107.5 \\
\hline$C(1)-C(14)-C(13)$ & $113.7(2)$ & $\mathrm{C}(1)-\mathrm{C}(14)-\mathrm{H}(14 \mathrm{~A})$ & 108.8 \\
\hline C(13)-C(14)-H(14A) & 108.8 & $\mathrm{C}(1)-\mathrm{C}(14)-\mathrm{H}(14 \mathrm{~B})$ & 108.8 \\
\hline C(13)-C(14)-H(14B) & 108.8 & $\mathrm{H}(14 \mathrm{~A})-\mathrm{C}(14)-\mathrm{H}(14 \mathrm{~B})$ & 107.7 \\
\hline $\mathrm{O}(7)-\mathrm{C}(15)-\mathrm{O}(6)$ & $124.8(3)$ & $\mathrm{O}(7)-\mathrm{C}(15)-\mathrm{C}(2)$ & $123.8(3)$ \\
\hline $\mathrm{O}(6)-\mathrm{C}(15)-\mathrm{C}(2)$ & $111.5(2)$ & $\mathrm{O}(6)-\mathrm{C}(16)-\mathrm{H}(16 \mathrm{~A})$ & 109.5 \\
\hline $\mathrm{O}(6)-\mathrm{C}(16)-\mathrm{H}(16 \mathrm{~B})$ & 109.5 & $\mathrm{H}(16 \mathrm{~A})-\mathrm{C}(16)-\mathrm{H}(16 \mathrm{~B})$ & 109.5 \\
\hline $\mathrm{O}(6)-\mathrm{C}(16)-\mathrm{H}(16 \mathrm{C})$ & 109.5 & H(16A)-C(16)-H(16C) & 109.5 \\
\hline H(16B)-C(16)-H(16C) & 109.5 & $\mathrm{O}(9)-\mathrm{C}(17)-\mathrm{O}(8)$ & $125.5(3)$ \\
\hline $\mathrm{O}(9)-\mathrm{C}(17)-\mathrm{C}(3)$ & 121.8(3) & $\mathrm{O}(8)-\mathrm{C}(17)-\mathrm{C}(3)$ & $112.6(2)$ \\
\hline $\mathrm{O}(8)-\mathrm{C}(18)-\mathrm{H}(18 \mathrm{~A})$ & 109.5 & $\mathrm{O}(8)-\mathrm{C}(18)-\mathrm{H}(18 \mathrm{~B})$ & 109.5 \\
\hline $\mathrm{H}(18 \mathrm{~A})-\mathrm{C}(18)-\mathrm{H}(18 \mathrm{~B})$ & 109.5 & $\mathrm{O}(8)-\mathrm{C}(18)-\mathrm{H}(18 \mathrm{C})$ & 109.5 \\
\hline H(18A)-C(18)-H(18C) & 109.5 & H(18B)-C(18)-H(18C) & 109.5 \\
\hline$C(20)-C(19)-C(10)$ & $112.2(2)$ & $\mathrm{C}(20)-\mathrm{C}(19)-\mathrm{H}(19 \mathrm{~A})$ & 109.2 \\
\hline C(10)-C(19)-H(19A) & 109.2 & C(20)-C(19)-H(19B) & 109.2 \\
\hline C(10)-C(19)-H(19B) & 109.2 & $\mathrm{H}(19 \mathrm{~A})-\mathrm{C}(19)-\mathrm{H}(19 \mathrm{~B})$ & 107.9 \\
\hline$C(25)-C(20)-C(21)$ & 117.7(3) & $\mathrm{C}(25)-\mathrm{C}(20)-\mathrm{C}(19)$ & $120.8(3)$ \\
\hline$C(21)-C(20)-C(19)$ & 121.6(3) & $\mathrm{C}(20)-\mathrm{C}(21)-\mathrm{C}(22)$ & $119.7(4)$ \\
\hline $\mathrm{C}(20)-\mathrm{C}(21)-\mathrm{H}(21)$ & 120.2 & $\mathrm{C}(22)-\mathrm{C}(21)-\mathrm{H}(21)$ & 120.2 \\
\hline$C(23)-C(22)-C(21)$ & $119.2(4)$ & $\mathrm{C}(23)-\mathrm{C}(22)-\mathrm{H}(22)$ & 120.4 \\
\hline $\mathrm{C}(21)-\mathrm{C}(22)-\mathrm{H}(22)$ & 120.4 & $\mathrm{C}(24)-\mathrm{C}(23)-\mathrm{C}(22)$ & $120.7(4)$ \\
\hline $\mathrm{C}(24)-\mathrm{C}(23)-\mathrm{H}(23)$ & 119.6 & $\mathrm{C}(22)-\mathrm{C}(23)-\mathrm{H}(23)$ & 119.6 \\
\hline$C(23)-C(24)-C(25)$ & 119.3(5) & $\mathrm{C}(23)-\mathrm{C}(24)-\mathrm{H}(24)$ & 120.3 \\
\hline $\mathrm{C}(25)-\mathrm{C}(24)-\mathrm{H}(24)$ & 120.3 & $C(24)-C(25)-C(20)$ & $123.3(4)$ \\
\hline $\mathrm{C}(24)-\mathrm{C}(25)-\mathrm{H}(25)$ & 118.3 & $\mathrm{C}(20)-\mathrm{C}(25)-\mathrm{H}(25)$ & 118.3 \\
\hline $\mathrm{O}(5)-\mathrm{C}(26)-\mathrm{C}(12)$ & $108.9(2)$ & $\mathrm{O}(5)-\mathrm{C}(26)-\mathrm{H}(26 \mathrm{~A})$ & 109.9 \\
\hline $\mathrm{C}(12)-\mathrm{C}(26)-\mathrm{H}(26 \mathrm{~A})$ & 109.9 & $\mathrm{O}(5)-\mathrm{C}(26)-\mathrm{H}(26 \mathrm{~B})$ & 109.9 \\
\hline C(12)-C(26)-H(26B) & 109.9 & $\mathrm{H}(26 \mathrm{~A})-\mathrm{C}(26)-\mathrm{H}(26 \mathrm{~B})$ & 108.3 \\
\hline
\end{tabular}


Table 4. Anisotropic displacement parameters $\left(\AA^{2} \times 10^{3}\right)$ for sls87. The anisotropic displacement factor exponent takes the form: $-2 \pi^{2}\left[h^{2} a^{* 2} U^{11}+\ldots+2 h \mathrm{k} \mathrm{a}^{*} \mathrm{~b}^{*} \mathrm{U}^{12}\right]$

\begin{tabular}{|c|c|c|c|c|c|c|}
\hline & $\mathrm{U}^{11}$ & $\mathrm{U}^{22}$ & $\mathrm{U}^{33}$ & $\mathrm{U}^{23}$ & $\mathrm{U}^{13}$ & $\mathrm{U}^{12}$ \\
\hline $\mathrm{O}(1)$ & $37(1)$ & $24(1)$ & $34(1)$ & $2(1)$ & $8(1)$ & $-1(1)$ \\
\hline $\mathrm{O}(2)$ & $52(1)$ & $25(1)$ & $42(1)$ & $-2(1)$ & $16(1)$ & $-2(1)$ \\
\hline $\mathrm{O}(3)$ & $46(1)$ & $29(1)$ & $43(1)$ & $-5(1)$ & $9(1)$ & $6(1)$ \\
\hline $\mathrm{O}(4)$ & $19(1)$ & $36(1)$ & $41(1)$ & $15(1)$ & $1(1)$ & $3(1)$ \\
\hline $\mathrm{O}(5)$ & $26(1)$ & $27(1)$ & $87(2)$ & $-8(1)$ & $2(1)$ & $-5(1)$ \\
\hline $\mathrm{O}(6)$ & $56(1)$ & $36(1)$ & $40(1)$ & $5(1)$ & $12(1)$ & $-12(1)$ \\
\hline $\mathrm{O}(7)$ & $56(1)$ & $61(2)$ & $65(1)$ & $2(1)$ & 31(1) & $-7(1)$ \\
\hline $\mathrm{O}(8)$ & $37(1)$ & $68(2)$ & $34(1)$ & $-16(1)$ & $-6(1)$ & $16(1)$ \\
\hline $\mathrm{O}(9)$ & $65(2)$ & $61(1)$ & $28(1)$ & $-2(1)$ & $-3(1)$ & $27(1)$ \\
\hline $\mathrm{N}(1)$ & $29(1)$ & $25(1)$ & $51(1)$ & $1(1)$ & $6(1)$ & $-1(1)$ \\
\hline $\mathrm{N}(2)$ & $29(1)$ & $25(1)$ & $46(1)$ & $5(1)$ & $2(1)$ & $0(1)$ \\
\hline N(3) & $27(1)$ & $23(1)$ & $41(1)$ & $3(1)$ & $3(1)$ & $-2(1)$ \\
\hline $\mathrm{N}(4)$ & 18(1) & $29(1)$ & $29(1)$ & $6(1)$ & $0(1)$ & $2(1)$ \\
\hline N(5) & $17(1)$ & $32(1)$ & $36(1)$ & $8(1)$ & 1(1) & $1(1)$ \\
\hline $\mathrm{C}(1)$ & $32(1)$ & $29(1)$ & $27(1)$ & $5(1)$ & $-2(1)$ & $-1(1)$ \\
\hline$C(2)$ & $30(1)$ & $36(2)$ & $26(1)$ & $2(1)$ & $-3(1)$ & $-4(1)$ \\
\hline C(3) & $30(1)$ & $35(2)$ & $25(1)$ & $0(1)$ & $-1(1)$ & 2(1) \\
\hline$C(4)$ & $31(1)$ & $29(1)$ & 29(1) & $-3(1)$ & $1(1)$ & $3(1)$ \\
\hline$C(5)$ & $34(1)$ & $30(2)$ & $29(1)$ & $-2(1)$ & $0(1)$ & $0(1)$ \\
\hline$C(6)$ & $49(2)$ & $30(2)$ & $47(2)$ & $-4(1)$ & $13(2)$ & $-6(1)$ \\
\hline$C(7)$ & $35(2)$ & $19(1)$ & $43(2)$ & $-2(1)$ & $5(1)$ & $-5(1)$ \\
\hline C(8) & $32(1)$ & $26(1)$ & $38(1)$ & $4(1)$ & $-1(1)$ & $1(1)$ \\
\hline C(9) & $27(1)$ & $33(2)$ & $38(1)$ & $4(1)$ & $5(1)$ & $-3(1)$ \\
\hline$C(10)$ & $20(1)$ & $32(1)$ & $28(1)$ & $7(1)$ & $0(1)$ & $-3(1)$ \\
\hline$C(11)$ & $22(1)$ & $22(1)$ & $25(1)$ & $-2(1)$ & $0(1)$ & $-1(1)$ \\
\hline$C(12)$ & $20(1)$ & $20(1)$ & $32(1)$ & $3(1)$ & $4(1)$ & $1(1)$ \\
\hline$C(13)$ & $29(1)$ & $23(1)$ & $34(1)$ & 1(1) & $4(1)$ & $3(1)$ \\
\hline$C(14)$ & $38(2)$ & $29(2)$ & $33(1)$ & $6(1)$ & $7(1)$ & $0(1)$ \\
\hline$C(15)$ & $36(2)$ & $48(2)$ & $28(1)$ & $3(1)$ & $2(1)$ & $-7(1)$ \\
\hline$C(16)$ & $61(2)$ & $51(2)$ & $48(2)$ & $16(2)$ & $-2(2)$ & $-22(2)$ \\
\hline$C(17)$ & $32(2)$ & $37(2)$ & $32(1)$ & 1(1) & $6(1)$ & $0(1)$ \\
\hline
\end{tabular}




\begin{tabular}{lcccccc}
$\mathrm{C}(18)$ & $50(2)$ & $85(3)$ & $43(2)$ & $-25(2)$ & $-6(2)$ & $29(2)$ \\
$\mathrm{C}(19)$ & $29(1)$ & $40(2)$ & $30(1)$ & $8(1)$ & $4(1)$ & $-2(1)$ \\
$\mathrm{C}(20)$ & $37(2)$ & $40(2)$ & $27(1)$ & $3(1)$ & $9(1)$ & $2(1)$ \\
$\mathrm{C}(21)$ & $89(3)$ & $48(2)$ & $46(2)$ & $9(2)$ & $7(2)$ & $18(2)$ \\
$\mathrm{C}(22)$ & $158(5)$ & $35(2)$ & $75(3)$ & $10(2)$ & $47(3)$ & $15(3)$ \\
$\mathrm{C}(23)$ & $118(4)$ & $80(4)$ & $83(3)$ & $-34(3)$ & $38(3)$ & $-34(3)$ \\
$\mathrm{C}(24)$ & $75(3)$ & $98(4)$ & $90(3)$ & $-42(3)$ & $-3(3)$ & $-15(3)$ \\
$\mathrm{C}(25)$ & $52(2)$ & $61(2)$ & $54(2)$ & $-17(2)$ & $-2(2)$ & $0(2)$ \\
$\mathrm{C}(26)$ & $24(1)$ & $29(1)$ & $39(1)$ & $2(1)$ & $-2(1)$ & $-1(1)$ \\
\hline
\end{tabular}


Table 5. Hydrogen coordinates ( x 104) and isotropic displacement parameters $\left(\AA^{2} \times 10^{3}\right)$ for sls87.

\begin{tabular}{|c|c|c|c|c|}
\hline & $\mathrm{x}$ & $\mathrm{y}$ & $\mathrm{z}$ & $\mathrm{U}(\mathrm{eq})$ \\
\hline $\mathrm{H}(5 \mathrm{~B})$ & 6121 & 3019 & 7516 & 69 \\
\hline $\mathrm{H}(4)$ & -3550 & 1012 & 7955 & 30 \\
\hline $\mathrm{H}(5)$ & -1745 & 1692 & 7415 & 34 \\
\hline $\mathrm{H}(6 \mathrm{~A})$ & 2290 & -1231 & 6610 & 50 \\
\hline $\mathrm{H}(6 \mathrm{~B})$ & -482 & -921 & 6448 & 50 \\
\hline $\mathrm{H}(8)$ & -3320 & -276 & 7228 & 38 \\
\hline $\mathrm{H}(9 \mathrm{~A})$ & -3122 & -737 & 8508 & 39 \\
\hline $\mathrm{H}(9 \mathrm{~B})$ & -4991 & -323 & 8147 & 39 \\
\hline $\mathrm{H}(10)$ & -228 & 234 & 8527 & 32 \\
\hline $\mathrm{H}(12)$ & 3421 & 1516 & 7208 & 29 \\
\hline $\mathrm{H}(13 \mathrm{~A})$ & 39 & 1861 & 6633 & 34 \\
\hline $\mathrm{H}(13 \mathrm{~B})$ & 275 & 2638 & 6846 & 34 \\
\hline $\mathrm{H}(14 \mathrm{~A})$ & 2820 & 2679 & 6191 & 40 \\
\hline $\mathrm{H}(14 \mathrm{~B})$ & 4874 & 2600 & 6615 & 40 \\
\hline $\mathrm{H}(16 \mathrm{~A})$ & 8329 & 3358 & 5164 & 80 \\
\hline $\mathrm{H}(16 \mathrm{~B})$ & 10711 & 3228 & 5520 & 80 \\
\hline $\mathrm{H}(16 \mathrm{C})$ & 8484 & 3781 & 5656 & 80 \\
\hline $\mathrm{H}(18 \mathrm{~A})$ & 11584 & -1 & 4884 & 89 \\
\hline $\mathrm{H}(18 \mathrm{~B})$ & 9579 & -627 & 4990 & 89 \\
\hline $\mathrm{H}(18 \mathrm{C})$ & 12318 & -603 & 5260 & 89 \\
\hline $\mathrm{H}(19 \mathrm{~A})$ & -5482 & 630 & 8758 & 40 \\
\hline $\mathrm{H}(19 \mathrm{~B})$ & -3598 & 197 & 9105 & 40 \\
\hline $\mathrm{H}(21)$ & -5224 & 1898 & 8742 & 73 \\
\hline $\mathrm{H}(22)$ & -3403 & 2954 & 9051 & 107 \\
\hline $\mathrm{H}(23)$ & 177 & 2891 & 9557 & 112 \\
\hline $\mathrm{H}(24)$ & 1956 & 1820 & 9753 & 106 \\
\hline $\mathrm{H}(25)$ & 241 & 807 & 9441 & 67 \\
\hline $\mathrm{H}(26 \mathrm{~A})$ & 2144 & 2861 & 7604 & 37 \\
\hline $\mathrm{H}(26 \mathrm{~B})$ & 3275 & 2240 & 7932 & 37 \\
\hline
\end{tabular}


Table 6. Torsion angles $\left[^{\circ}\right]$ for sls87.

\begin{tabular}{|c|c|}
\hline $\mathrm{C}(7)-\mathrm{N}(1)-\mathrm{N}(2)-\mathrm{N}(3)$ & $0.0(3)$ \\
\hline N(1)-N(2)-N(3)-C(8) & $-0.3(3)$ \\
\hline $\mathrm{N}(1)-\mathrm{N}(2)-\mathrm{N}(3)-\mathrm{C}(9)$ & $-172.8(2)$ \\
\hline $\mathrm{C}(4)-\mathrm{O}(1)-\mathrm{C}(1)-\mathrm{C}(2)$ & $0.0(3)$ \\
\hline $\mathrm{C}(4)-\mathrm{O}(1)-\mathrm{C}(1)-\mathrm{C}(14)$ & $177.9(2)$ \\
\hline $\mathrm{O}(1)-\mathrm{C}(1)-\mathrm{C}(2)-\mathrm{C}(3)$ & $0.6(3)$ \\
\hline $\mathrm{C}(14)-\mathrm{C}(1)-\mathrm{C}(2)-\mathrm{C}(3)$ & $-176.7(3)$ \\
\hline $\mathrm{O}(1)-\mathrm{C}(1)-\mathrm{C}(2)-\mathrm{C}(15)$ & $-175.7(2)$ \\
\hline$C(14)-C(1)-C(2)-C(15)$ & $7.0(5)$ \\
\hline $\mathrm{C}(1)-\mathrm{C}(2)-\mathrm{C}(3)-\mathrm{C}(4)$ & $-1.0(3)$ \\
\hline C(15)-C(2)-C(3)-C(4) & $175.5(2)$ \\
\hline $\mathrm{C}(1)-\mathrm{C}(2)-\mathrm{C}(3)-\mathrm{C}(17)$ & 169.5(3) \\
\hline C(15)-C(2)-C(3)-C(17) & $-14.0(4)$ \\
\hline $\mathrm{C}(2)-\mathrm{C}(3)-\mathrm{C}(4)-\mathrm{O}(1)$ & $1.0(3)$ \\
\hline $\mathrm{C}(17)-\mathrm{C}(3)-\mathrm{C}(4)-\mathrm{O}(1)$ & $-169.8(2)$ \\
\hline C(2)-C(3)-C(4)-C(5) & 177.3(3) \\
\hline C(17)-C(3)-C(4)-C(5) & $6.5(5)$ \\
\hline $\mathrm{C}(1)-\mathrm{O}(1)-\mathrm{C}(4)-\mathrm{C}(3)$ & $-0.7(3)$ \\
\hline $\mathrm{C}(1)-\mathrm{O}(1)-\mathrm{C}(4)-\mathrm{C}(5)$ & $-177.6(2)$ \\
\hline $\mathrm{C}(6)-\mathrm{O}(2)-\mathrm{C}(5)-\mathrm{O}(3)$ & $-7.9(4)$ \\
\hline $\mathrm{C}(6)-\mathrm{O}(2)-\mathrm{C}(5)-\mathrm{C}(4)$ & $173.0(2)$ \\
\hline $\mathrm{C}(3)-\mathrm{C}(4)-\mathrm{C}(5)-\mathrm{O}(3)$ & $3.6(5)$ \\
\hline $\mathrm{O}(1)-\mathrm{C}(4)-\mathrm{C}(5)-\mathrm{O}(3)$ & 179.7(3) \\
\hline $\mathrm{C}(3)-\mathrm{C}(4)-\mathrm{C}(5)-\mathrm{O}(2)$ & $-177.3(3)$ \\
\hline $\mathrm{O}(1)-\mathrm{C}(4)-\mathrm{C}(5)-\mathrm{O}(2)$ & $-1.2(3)$ \\
\hline $\mathrm{C}(5)-\mathrm{O}(2)-\mathrm{C}(6)-\mathrm{C}(7)$ & $155.0(2)$ \\
\hline N(2)-N(1)-C(7)-C(8) & $0.3(3)$ \\
\hline N(2)-N(1)-C(7)-C(6) & $178.9(2)$ \\
\hline $\mathrm{O}(2)-\mathrm{C}(6)-\mathrm{C}(7)-\mathrm{N}(1)$ & $-98.1(3)$ \\
\hline $\mathrm{O}(2)-\mathrm{C}(6)-\mathrm{C}(7)-\mathrm{C}(8)$ & $80.1(4)$ \\
\hline $\mathrm{N}(2)-\mathrm{N}(3)-\mathrm{C}(8)-\mathrm{C}(7)$ & $0.4(3)$ \\
\hline $\mathrm{C}(9)-\mathrm{N}(3)-\mathrm{C}(8)-\mathrm{C}(7)$ & $172.1(2)$ \\
\hline $\mathrm{N}(1)-\mathrm{C}(7)-\mathrm{C}(8)-\mathrm{N}(3)$ & $-0.4(3)$ \\
\hline $\mathrm{C}(6)-\mathrm{C}(7)-\mathrm{C}(8)-\mathrm{N}(3)$ & $-178.8(3)$ \\
\hline
\end{tabular}




\begin{tabular}{|c|c|}
\hline $\mathrm{C}(8)-\mathrm{N}(3)-\mathrm{C}(9)-\mathrm{C}(10)$ & $-88.2(3)$ \\
\hline $\mathrm{N}(2)-\mathrm{N}(3)-\mathrm{C}(9)-\mathrm{C}(10)$ & $82.9(3)$ \\
\hline $\mathrm{C}(11)-\mathrm{N}(4)-\mathrm{C}(10)-\mathrm{C}(9)$ & $-107.4(3)$ \\
\hline $\mathrm{C}(11)-\mathrm{N}(4)-\mathrm{C}(10)-\mathrm{C}(19)$ & $130.4(2)$ \\
\hline $\mathrm{N}(3)-\mathrm{C}(9)-\mathrm{C}(10)-\mathrm{N}(4)$ & $58.4(3)$ \\
\hline N(3)-C(9)-C(10)-C(19) & $-178.3(2)$ \\
\hline $\mathrm{C}(12)-\mathrm{N}(5)-\mathrm{C}(11)-\mathrm{O}(4)$ & $-7.4(4)$ \\
\hline $\mathrm{C}(12)-\mathrm{N}(5)-\mathrm{C}(11)-\mathrm{N}(4)$ & $173.9(2)$ \\
\hline $\mathrm{C}(10)-\mathrm{N}(4)-\mathrm{C}(11)-\mathrm{O}(4)$ & $3.8(4)$ \\
\hline $\mathrm{C}(10)-\mathrm{N}(4)-\mathrm{C}(11)-\mathrm{N}(5)$ & $-177.5(2)$ \\
\hline $\mathrm{C}(11)-\mathrm{N}(5)-\mathrm{C}(12)-\mathrm{C}(26)$ & $-78.7(3)$ \\
\hline $\mathrm{C}(11)-\mathrm{N}(5)-\mathrm{C}(12)-\mathrm{C}(13)$ & $156.8(2)$ \\
\hline N(5)-C(12)-C(13)-C(14) & $-162.0(2)$ \\
\hline $\mathrm{C}(26)-\mathrm{C}(12)-\mathrm{C}(13)-\mathrm{C}(14)$ & $76.2(3)$ \\
\hline $\mathrm{O}(1)-\mathrm{C}(1)-\mathrm{C}(14)-\mathrm{C}(13)$ & $-6.2(3)$ \\
\hline $\mathrm{C}(2)-\mathrm{C}(1)-\mathrm{C}(14)-\mathrm{C}(13)$ & $170.9(3)$ \\
\hline$C(12)-C(13)-C(14)-C(1)$ & $75.9(3)$ \\
\hline $\mathrm{C}(16)-\mathrm{O}(6)-\mathrm{C}(15)-\mathrm{O}(7)$ & $2.0(4)$ \\
\hline$C(16)-O(6)-C(15)-C(2)$ & $-179.5(2)$ \\
\hline $\mathrm{C}(1)-\mathrm{C}(2)-\mathrm{C}(15)-\mathrm{O}(7)$ & $175.4(3)$ \\
\hline C(3)-C(2)-C(15)-O(7) & $-0.3(4)$ \\
\hline $\mathrm{C}(1)-\mathrm{C}(2)-\mathrm{C}(15)-\mathrm{O}(6)$ & $-3.1(4)$ \\
\hline C(3)-C(2)-C(15)-O(6) & $-178.8(2)$ \\
\hline $\mathrm{C}(18)-\mathrm{O}(8)-\mathrm{C}(17)-\mathrm{O}(9)$ & $-1.7(4)$ \\
\hline $\mathrm{C}(18)-\mathrm{O}(8)-\mathrm{C}(17)-\mathrm{C}(3)$ & 176.5(3) \\
\hline $\mathrm{C}(4)-\mathrm{C}(3)-\mathrm{C}(17)-\mathrm{O}(9)$ & $99.6(4)$ \\
\hline C(2)-C(3)-C(17)-O(9) & $-69.1(4)$ \\
\hline $\mathrm{C}(4)-\mathrm{C}(3)-\mathrm{C}(17)-\mathrm{O}(8)$ & $-78.7(3)$ \\
\hline $\mathrm{C}(2)-\mathrm{C}(3)-\mathrm{C}(17)-\mathrm{O}(8)$ & $112.7(3)$ \\
\hline N(4)-C(10)-C(19)-C(20) & $-58.5(3)$ \\
\hline C(9)-C(10)-C(19)-C(20) & $178.5(2)$ \\
\hline C(10)-C(19)-C(20)-C(25) & $-81.4(3)$ \\
\hline C(10)-C(19)-C(20)-C(21) & $98.5(3)$ \\
\hline $\mathrm{C}(25)-\mathrm{C}(20)-\mathrm{C}(21)-\mathrm{C}(22)$ & $1.6(5)$ \\
\hline C(19)-C(20)-C(21)-C(22) & $-178.3(3)$ \\
\hline $\mathrm{C}(20)-\mathrm{C}(21)-\mathrm{C}(22)-\mathrm{C}(23)$ & $-1.2(6)$ \\
\hline
\end{tabular}




$\begin{array}{lc}\mathrm{C}(21)-\mathrm{C}(22)-\mathrm{C}(23)-\mathrm{C}(24) & 0.0(7) \\ \mathrm{C}(22)-\mathrm{C}(23)-\mathrm{C}(24)-\mathrm{C}(25) & 0.6(7) \\ \mathrm{C}(23)-\mathrm{C}(24)-\mathrm{C}(25)-\mathrm{C}(20) & -0.2(6) \\ \mathrm{C}(21)-\mathrm{C}(20)-\mathrm{C}(25)-\mathrm{C}(24) & -1.0(5) \\ \mathrm{C}(19)-\mathrm{C}(20)-\mathrm{C}(25)-\mathrm{C}(24) & 179.0(3) \\ \mathrm{N}(5)-\mathrm{C}(12)-\mathrm{C}(26)-\mathrm{O}(5) & 162.90(19) \\ \mathrm{C}(13)-\mathrm{C}(12)-\mathrm{C}(26)-\mathrm{O}(5) & -75.3(3)\end{array}$

Symmetry transformations used to generate equivalent atoms: 
Table 7. Hydrogen bonds for sls87 $\left[\AA\right.$ and $\left.{ }^{\circ}\right]$.

\begin{tabular}{lcccc}
\hline D-H...A & d(D-H) & d(H...A & $d(D . . A)$ & $<($ DHA $)$ \\
\hline $\mathrm{N}(5)-H(5) \ldots \mathrm{O}(5) \# 1$ & 0.88 & 2.14 & $2.867(3)$ & 140.0 \\
$\mathrm{~N}(4)-\mathrm{H}(4) \ldots \mathrm{O}(4) \# 1$ & 0.88 & 2.17 & $2.919(3)$ & 142.3 \\
$\mathrm{O}(5)-\mathrm{H}(5 \mathrm{~B}) \ldots \mathrm{N}(1) \# 2$ & 0.84 & 2.01 & $2.782(3)$ & 153.4 \\
& & & & \\
\hline
\end{tabular}

Symmetry transformations used to generate equivalent atoms:

$\# 1 \mathrm{x}-1, \mathrm{y}, \mathrm{z} \quad \# 2-\mathrm{x}+1, \mathrm{y}+1 / 2,-\mathrm{z}+3 / 2$ 